Institut für Allgemeine Mechanik der RWTH Aachen

DISSERTATION

\title{
Remodeling of Soft Tissues due to Cell Activity
}

Jeong-Hun Yi 

,Remodeling of Soft Tissues due to Cell Activity“

\author{
Von der Fakultät für Maschinenwesen \\ der Rheinisch-Westfälischen Technischen Hochschule Aachen \\ zur Erlangung des akademischen Grades eines Doktors der \\ Ingenieurwissenschaften genehmigte Dissertation
}

vorgelegt von

Jeong-Hun Yi

Berichter: Universitätsprofessor Dr.-Ing. Dieter Weichert

Professor Dr.-Ing. Marcus Stoffel

Tag der mündlichen Prüfung: 04.06.2013

Diese Dissertation ist auf den Internetseiten der Hochschulbibliothek online verfügbar. 



\section{Acknowledgements}

This thesis is a result of my activities as a research scientist in the Institute of General Mechanics (IAM) at the RWTH Aachen University. I am thankful to the head of the institute, Univ.-Prof. Dr.-Ing. Dieter Weichert, for his kind guidance of my research. He gave me teaching and research opportunities and broadened my perspective of thought from merely stating equations to bridging the gap between theories and experiments.

To my biomechanics group leader, Professor Dr.-Ing. Marcus Stoffel, I express my deepest gratitude. He led all aspects of my research and spared no effort to help me. His constant support and advice allowed me to understand what biomechanics is all about.

I thank Professor Dr.-Ing. Jochen Büchs for accepting the position as president of the examination board for my defense.

I gratefully acknowledge the following colleagues. Dr. rer. nat. Michael Ban cleared up any confusion, whenever I had a mathematical question.

Dr.-Ing. Russell Todres and Dr.-Ing. Heiko Topol always willingly helped me when I needed the help of native speakers of English and German.

M. Sc. Bei Zhou cooperated with me in experiments and simulations. With her assistance, I was able to save time in numerical calculations.

In addition, my comrade and former officemate, Dipl.-Ing. Malte Strampe, provided helpful advice and support.

Finally, I express my deepest gratitude to my parents, parents-in-law, my two children, Yeo-Jun and Yeo-Eun, and my wife, Su-Mi. 



\begin{abstract}
This work deals with the analysis of the modified material properties of cellular tissues. For the experimental study, cell-seeded condensed collagen gel specimens were stimulated mechanically in bioreactors for four weeks. To follow the Young's modulus modification (remodeling), a compression test was performed every week. A constitutive equation is proposed for the remodeling effect of the cellular tissue, and a remodeling velocity parameter is identified.
\end{abstract}

Zusammenfassung: Die vorliegende Arbeit behandelt die Untersuchung der modifizierten Materialeigenschaften von der Zellengeweben. Für die experimentelle Untersuchung wurden Proben aus zellbesiedelten verdichteten Kollagengel im Bioreaktor für vier Wochen stimuliert. Jede Woche wurde ein Drucktest durchgeführt, um die Änderung des Elastizitätsmoduls zu folgen. Für die Remodeling der Zellengeweben wird ein Stoffgesetz vorgeschlagen, und ein Parameter der Umbaugeschwindigkeit wird identifiziert. 



\section{Contents}

List of Figures $\quad$ vi

List of Tables $\quad$ vii

Nomenclature $\quad$ ix

1 Introduction $\quad 1$

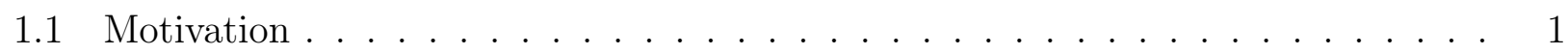

1.2 Recent work . . . . . . . . . . . . . . . . . . . 3

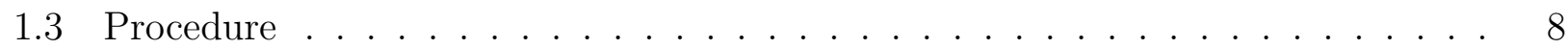

2 Articular cartilage from a medical viewpoint $\quad 9$

2.1 Kinds of cartilage . . . . . . . . . . . . . . . . . . 9

2.2 Structure of articular cartilage . . . . . . . . . . . . . . . 10

2.3 Mechanical properties of articular cartilage . . . . . . . . . . . . . . 14

2.4 Injury of articular cartilage . . . . . . . . . . . . . . . 14

2.5 Reparative surgery of articular cartilage . . . . . . . . . . . . . 16

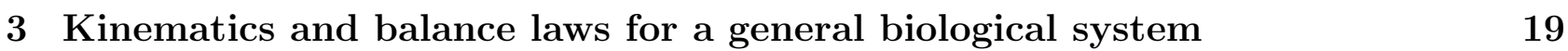

3.1 Kinematics of growth . . . . . . . . . . . . . . . . . 21

3.2 Balance laws for an open system . . . . . . . . . . . . . . . . . . 25

3.2 .1 Balance of mass . . . . . . . . . . . . . . . . . . . 25

3.2 .2 Balance of linear momentum . . . . . . . . . . . . . . . 27

3.2 .3 Balance of angular momentum . . . . . . . . . . . . . . 31

3.2 .4 Balance of energy . . . . . . . . . . . . . . . . 36

3.2 .5 Entropy inequality . . . . . . . . . . . . . . . . . 40 
3.3 Reduction from an open system to a closed system . . . . . . . . . . . . . . 43

4 Constitutive modeling of soft tissue 45

4.1 Mechanical behaviors of soft tissue . . . . . . . . . . . . . . . . 45

4.1 .1 Solid behavior . . . . . . . . . . . . . . . . . 45

$4.1 .2 \quad$ Fiber-reinforced solid behavior . . . . . . . . . . . . . . . . 50

4.1.3 Fluid behavior and fluid-solid interaction . . . . . . . . . . . . . . . 51

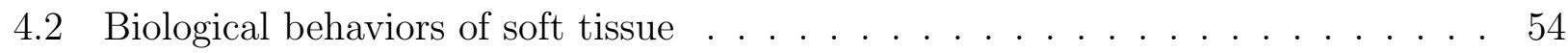

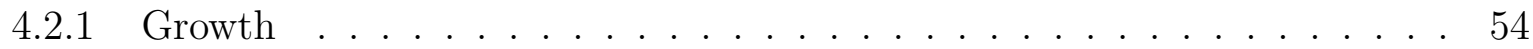

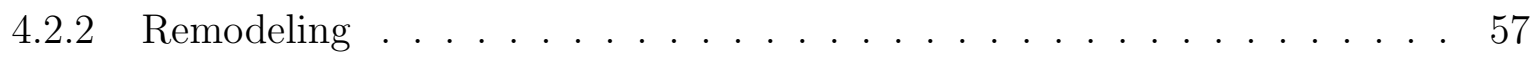

4.3 Proposed model: diffusive-remodeled materials . . . . . . . . . . . . . . . . . . 61

4.3 .1 Assumptions . . . . . . . . . . . . . . . . . . . 61

4.3.2 Viscoelastic solid model . . . . . . . . . . . . . . . . . . . . . 62

4.3.2.1 Objectivity of the constitutive equation . . . . . . . . 65

4.3.2.2 Elastic stress tensor and viscous stress tensor . . . . . . . . . 66

4.3.2.3 Elasticity tensor and viscosity tensor . . . . . . . . . . . . 71

4.3 .3 Simplifications . . . . . . . . . . . . . . . . . . . . . . 79

4.3.4 Diffusive-remodeled materials: IAM model . . . . . . . . . . . . . . . . . 80

4.3.5 Summary of the IAM model . . . . . . . . . . . . . . . . . . . 84

4.3.6 Comparison of KLM model and IAM model . . . . . . . . . . . . . . . 85

$\begin{array}{llr}5 & \text { Experiments } & 89\end{array}$

5.1 Cell-seeded condensed collagen gel . . . . . . . . . . . . . . . 89

5.2 Bioreactor . . . . . . . . . . . . . . . . . . . . 92

5.2 .1 Bioreactor system . . . . . . . . . . . . . . . . . . . 92

5.2 .2 Phenomena occuring in a bioreactor . . . . . . . . . . . . . 96

5.2 .3 Signals from bioreactors . . . . . . . . . . . . . . . . . 98

5.3 Mechanical tests for soft tissues . . . . . . . . . . . . . . . . . . 101

5.4 Compression test . . . . . . . . . . . . . . . . . . . . . . 104

5.4 .1 Compression testing . . . . . . . . . . . . . . . . 104

5.4.2 Signals from compression tests . . . . . . . . . . . . . . 105 
6 Numerical modeling and results $\quad 109$

6.1 Numerical model . . . . . . . . . . . . . . . . . . . . . 109

6.1.1 Short time-scale evolution . . . . . . . . . . . . . 113

6.1.2 Long time-scale evolution . . . . . . . . . . . . . . . . . 113

6.2 Numerical calculations . . . . . . . . . . . . . . . . . . 116

6.2.1 Parameter identification with compression test . . . . . . . . 116

6.2.2 Remodeling curve . . . . . . . . . . . . . . . . . . . . 121

6.2.3 Evaluation of elastic modulus change . . . . . . . . . . . . . . . 123

7 Conclusions $\quad 125$

7.1 Summary . . . . . . . . . . . . . . . . . . . 125

7.2 Future perspectives . . . . . . . . . . . . . . . . 127

$\begin{array}{lr}\text { A Tensors } & 129\end{array}$

A.1 Second-order tensors . . . . . . . . . . . . . . . . . . . . . . 129

A.2 Fourth-order tensors . . . . . . . . . . . . . . . . 134

A.3 Differentiation with respect to a tensor . . . . . . . . . . . . . 137

A.4 Some identities of tensor analysis . . . . . . . . . . . . . . . . . . 139

B Continuum mechanics 141

B.1 Kinematics of deformation . . . . . . . . . . . . . . . . . . . 141

B.2 Balance laws and Clausius-Duhem inequality . . . . . . . . . . . . 146

B.3 Constitutive theory . . . . . . . . . . . . . . . . . 149

$\begin{array}{ll}\text { C Appendix } & 157\end{array}$

C.1 Balance of mass in the current configuartion . . . . . . . . . . . . 157 


\section{List of Figures}

1.1 Articular cartilage lesion in knee $($ from $[5]) \ldots \ldots \ldots$. . . . . . . . . 1

1.2 Punching and filling of articular cartilage (from $[6]$ ) . . . . . . . . . . . 2

2.1 Structure of diarthrodial knee joint at different scales I (from [60]). . . . . . . . 10

2.2 Structure of diarthrodial joint at different scales II (from [62]) . . . . . . . . . 11

2.3 Structure of articular cartilage I (from [24]) . . . . . . . . . . . . . . . 12

2.4 Structure of articular cartilage II (from [24]) . . . . . . . . . . . . . . 13

2.5 Evolution of Osteoarthritis (from [1]) . . . . . . . . . . . . . 15

2.6 Optional caption for list of figures . . . . . . . . . . . . . . 16

2.7 Optional caption for list of figures . . . . . . . . . . . . . . . 17

3.1 Kinematics of growing body and residual stress. . . . . . . . . . . . . . . . 21

3.2 Different residual stresses. . . . . . . . . . . . . . . . . . . . . 22

3.3 Continuum tissue with source, flux and under stress. . . . . . . . . . . . 23

4.1 Precondition (Modified from Fung $[25]) \ldots \ldots \ldots$. . . . . . . . . . . . . 46

4.2 Configurations for a growing body (Modified from Rodriguez et al. [41]). . . . . 55

4.3 Adaptation rate depending on stimulus (Modified from Beaupré et al. [11]). . . . 59

4.4 Flowchart of elasticity remodeling . . . . . . . . . . . . . . . . . . 83

5.1 Optional caption for list of figures . . . . . . . . . . . . . . . . . . . 90

5.2 Optional caption for list of figures . . . . . . . . . . . . . . . . 91

5.3 Drawing of a bioreactor . . . . . . . . . . . . . . . . . 93

5.4 A photo of a bioreactor . . . . . . . . . . . . . . . . . 94

5.5 Optional caption for list of figures . . . . . . . . . . . . . . . 95 
5.6 Sketch of a bioreactor . . . . . . . . . . . . . . . . . 96

5.7 Histologies after 4 weeks stimulation in a bioreactor . . . . . . . . . . . . 97

5.8 Signals from a bioreactor, at first day . . . . . . . . . . . . . . . . . 98

5.9 Signals from a bioreactor, at sixteenth day . . . . . . . . . . . . . . . . 99

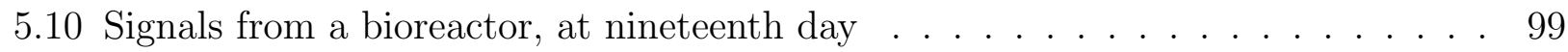

5.11 Specimens for uniaxial tension test and compression test . . . . . . . . . . 101

5.12 Various compression tests . . . . . . . . . . . . . . . . 102

5.13 Relaxation and creep . . . . . . . . . . . . . . . . . . . . 103

5.14 Compression test . . . . . . . . . . . . . . . . . . 104

5.15 Signals from a compression test, before bioreactor stimulation . . . . . . . . 105

5.16 Signals from a compression test, after a one-week bioreactor stimulation . . . . . 106

5.17 Signals from a compression test, after a two-week bioreactor stimulation . . . . . 106

5.18 Signals from a compression test, after a third-week bioreactor stimulation . . . . 107

5.19 Signals from a compression test, after a four-week bioreactor stimulation . . . . 107

6.1 Coordinate of the cylindrical specimen . . . . . . . . . . . . . . 111

6.2 Flowchart of Young's modulus evolution . . . . . . . . . . . . . . . . . . . 114

6.3 Cross section of the right side of a rotationally symmetric specimen . . . . . 117

6.4 Before bioreactor stimulation (initial state) . . . . . . . . . . . 117

6.5 After 1-week Bioreactor stimulation . . . . . . . . . . . . . . 118

6.6 After 2-week Bioreactor stimulation . . . . . . . . . . . . . . . . 118

6.7 After 3-week Bioreactor stimulation . . . . . . . . . . . . . . . . 119

6.8 After 4-week Bioreactor stimulation . . . . . . . . . . . . . . . . . . 119

6.9 Modification of E-modulus, D0 and D1 in bioreactor . . . . . . . . . . . . 120

6.10 Remodeling curve of elastic modulus $E_{p} \ldots \ldots \ldots$. . . . . . . . . . 121

6.11 Remodeling of elastic modulus $E_{p}$ in cross section . . . . . . . . . . . 122 


\section{List of Tables}

6.1 Modifications of elastic modulus and Diffusion parameters in a bioreactor . . . . 120

6.2 Modification of elastic modulus in 4 week bioreactor stimulation [MPa] . . . . 123

6.3 Modification of elastic modulus in 2 week bioreactor stimulation $[\mathrm{MPa}] \ldots$. . . 123 
LIST OF TABLES 


\section{Nomenclature}

\section{Tensors and matrices}

F

$\mathbf{F}^{\mathrm{g} i}$

$\tilde{\mathbf{F}}^{e i}$

$\overline{\mathbf{F}}^{\mathrm{e}}$

$\mathbf{F}^{\mathrm{e} i}$

C

$\operatorname{adj} \mathbf{M}$

$\mathbf{P}^{i}$

$\mathbf{P}$

$\mathrm{S}$

$\mathbf{S}^{\mathrm{e}}$

$\mathrm{S}^{\mathrm{v}}$

$\boldsymbol{\sigma}^{i}$

$\mathcal{E}$

$\mathbf{N}_{0}$

Q

I

0

I

(1)

$\mathbb{A}^{\mathrm{e}}$

$\mathbb{A}_{\text {crit }}^{e}$

Deformation gradient

Growth deformation gradient

Elastic deformation gradient of species $i$ introduced for compatibilty

Elastic deformation gradient of species $i$ due to external loading

Total elastic deformation gradient of species $i$

Right Cauchy-Green deformation tensor

Adjugate matrix of a matrix $\mathbf{M}$

Partial first Piola-Kirchhoff stress tensor of species $i$

First Piola-Kirchhoff stress tensor of system

Second Piola-Kirchhoff stress tensor of system

Elastic second Piola-Kirchhoff stress tensor

Viscous second Piola-Kirchhoff stress tensor

Cauchy stress tensor of species $i$

Permutation tensor

Structure tensor in reference configuration

Proper orthogonal tensor, $\mathbf{Q Q}^{\mathrm{T}}=\mathbf{I}$ and $\operatorname{det} \mathbf{Q}=1$

Second-order identity tensor

Second-order zero tensor

Fourth-order identity tensor, $\mathbb{I}=\mathbf{I} \otimes \mathbf{I}$

Fourth-order zero tensor

Fourth-order elasticity tensor

Fourth-order critical elasticity tensor 
$\mathbb{A}_{0}^{e} \quad$ Fourth-order initial elasticity tensor

$\mathbb{A}^{\mathrm{r}} \quad$ Fourth-order elasticity tensor increment due to remodeling

$\mathbb{A}^{\text {meg }} \quad$ Fourth-order modified elasticity tensor due to mass change (growth)

$\mathbb{A}^{\mathrm{v}} \quad$ Fourth-order viscosity tensor

$\mathbb{A}^{\text {mvg }} \quad$ Fourth-order modified viscosity tensor due to mass change (growth)

$\mathbf{A}^{\mathrm{e}} \quad$ Second-order tensor describing modified solid mass density depending on the deformation

$\mathrm{A}^{\mathrm{v}} \quad$ Second-order tensor describing modified solid mass density dependent on the deformation rate

$\mathbb{C}^{e} \quad$ Stiffness matrix in Voigt notation

$\dot{\mathbf{S}}^{\mathrm{d}} \quad$ Diffusive stress rate

$\sigma^{\mathrm{e}} \quad$ Elastic stress

$\sigma^{\mathrm{d}} \quad$ Diffusive stress

$\boldsymbol{\sigma}^{\mathrm{s}} \quad$ Stress of solid

$\boldsymbol{\sigma}^{\mathrm{f}} \quad$ Stress of fluid

\section{Vectors}

0

$\boldsymbol{X}$

$\boldsymbol{x}, \varphi$

$\boldsymbol{X}^{\mathrm{S}}$

$x^{\mathrm{S}}$

$N$

n

$\mathbf{n}_{0}$

$\mathbf{m}_{0}$

\section{Zero vector}

A position vector of a tissue material point in reference configuration

A position vector of a tissue material point in current configuration

A position vector of a solid material point in reference configuration

A position vector of a solid material point in current configuration

Unit normal vector in reference configuration

Unit normal vector in current configuration

Unit vector along fiber direction in reference configuration in case of

transversely isotropic material or

one of the two unit vectors in case of orthotropic material

Unit vector specufying one of the principal axes of orhthotropy in reference

configuration 
$M^{i} \quad$ Mass flux of species $i$ in reference configuration

$\boldsymbol{m}^{i} \quad$ Mass flux of species $i$ in current configuration

$\boldsymbol{T}^{i} \quad$ Partial surface traction of species $i$

$\boldsymbol{V} \quad$ Material velocity of system

$\boldsymbol{V}^{i} \quad$ Material velocity of species

$v$

Spatial velocity of system

$\boldsymbol{v}^{i}$

Spatial velocity of species

g

Body force per unit mass

$\mathbf{Q}^{i}$

Partial heat flux vector of species $i$

$\pi^{\mathrm{e}}$

Elastic part of the volumetric force

$\pi^{\mathrm{d}}$

Diffusive part of the volumetric force

$\pi^{\mathrm{df}}$

Diffusive volumetric force due to fluid

\section{Scalars}

$\vartheta_{\text {iso }}^{g i}$

$\eta_{\mathrm{tr}}^{\mathrm{g} i}$

$\vartheta_{\operatorname{tr}}^{\mathrm{g} i}$

$\eta_{\text {orth }}^{\mathrm{g} i}$

$\zeta_{\text {orth }}^{\text {i }}$

$\vartheta_{\text {orth }}^{\mathrm{g} i}$

$t$

$\rho_{\text {org }}^{i}(\boldsymbol{X})$

$\rho_{0}^{i}(\boldsymbol{X})$

$\rho^{i}(\boldsymbol{x}, t)$

$\rho_{0}$

$\Pi^{i}$

$\pi^{i}$

$J$

Stretch ratio in case of isotropic mass growth

Stretch ratio in the fiber direction in case of transversely isotropic mass growth

Stretch ratio in any orthogonal direction to the fiber direction in case of

transversely isotropic mass growth

Stretch ratio in the direction of $\mathbf{n}_{0}$ in case of orthotropic mass growth

Stretch ratio in the direction of $\mathbf{m}_{0}$ in case of orthotropic mass growth

Stretch ratio in the direction $\mathbf{n}_{0} \times \mathbf{m}_{0}$ in case of orthotropic mass growth

Time

Mass density of species $i$ in the undeformed state under stress-free condition

Mass density of species $i$ in reference configuration

Mass density of species $i$ in current configuration

Total mass density of the tissue in reference configuration

Mass source of species $i$ in reference configuration

Mass source of species $i$ in current configuration

Jacobian, local ratio of current to reference volume 
E Total energy of system

K Kinetic energy of system

$\mathcal{I} \quad$ Internal energy of system

$\mathcal{P} \quad$ Power of external forces

$\mathcal{Q} \quad$ Heat supply rate

$E^{i} \quad$ Internal energy per unit mass of species $i$

$R^{i} \quad$ Heat supply to species $i$ per unit mass

$H^{i} \quad$ Partial heat flux of species $i$ through the surface

$\tilde{E}^{i} \quad$ Energy transfer from other species to species $i$ per unit mass of species $i$

$N^{i} \quad$ Entropy per unit mass of species $i$

$\Theta \quad$ Temperature

$\Psi \quad$ Free energy density

$\Psi^{\mathrm{e}} \quad$ Elastic part of free energy density

$\Psi^{\mathrm{v}} \quad$ Viscous part of free energy density

$\mathcal{D} \quad$ Dissipation of energy

$\mathcal{D}_{\text {int }} \quad$ Internal dissipation (local entropy production)

$I_{\alpha}(\alpha=1, \cdots 5) \quad$ Strain invariants

$J_{\beta}(\beta=1, \cdots 12) \quad$ Invariants of strain rate

$\Psi_{\alpha}^{\mathrm{e}} \quad$ Partial derivative of $\Psi^{\mathrm{e}}$ with respect to $I_{\alpha}$

$\Psi_{\beta}^{\mathrm{v}} \quad$ Partial derivative of $\Psi^{\mathrm{v}}$ with respect to $J_{\beta}$

$\lambda^{\mathrm{Se}} \quad$ Change in mass source depending on strain energy

$\lambda^{\mathrm{Sv}} \quad$ Change in mass source depending on the viscous energy change

$D, D_{0}, D_{1} \quad$ Diffusion parameter

$E_{i} \quad$ Young's modulus along axis $X_{i}$

$\nu_{i j} \quad$ Poisson's ratio correspoding to a contraction in direction $X_{j}$ due to load in direction $X_{i}$

$G_{i j} \quad$ Shear modulus in direction $X_{j}$ on the plane whose nominal is in direction of $X_{i}$ 


\section{Abbreviations and operators}

$d(\bullet) \quad$ Differential of $(\bullet)$

LHS, RHS Left- and right-hand side of a equation

Index $i=\mathrm{S}, \mathrm{F}, 0 \quad$ Solid, fluid, and other product, respectively

Grad Referential (material) gradient operator

Div Referential (material) divergence operator

grad Spatial (eulerian) gradient operator

div Spatial (eulerian) divergence operator

$\operatorname{adj} \mathbf{T} \quad$ Ajugate matrix

$\frac{d}{d t}\{\bullet\}, \mathrm{D}_{t}\{\bullet\},\{\bullet\} \quad$ Material time derivative

$\frac{\partial}{\partial t}\{\bullet\} \quad$ Spatial time derivative

$\operatorname{sph}\{\bullet\} \quad$ Spherical part of tensor $\{\bullet\}$

$\operatorname{dev}\{\bullet\} \quad$ Deviatoric part of tensor $\{\bullet\}$ 
LIST OF TABLES 


\section{Chapter 1}

\section{Introduction}

\subsection{Motivation}

Articular cartilage problems in the knee joint are common in orthopedic surgery. Injuries, called lesions, often look like tears or potholes in the surface of the cartilage, Fig. 1.1.

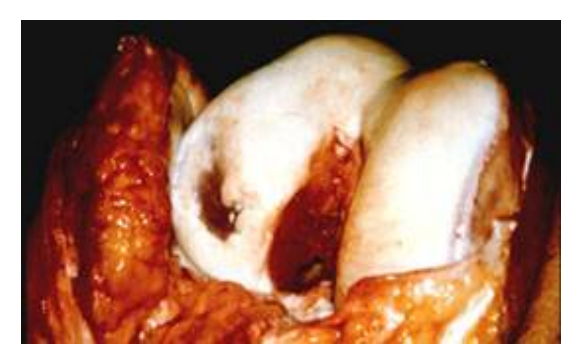

Figure 1.1: Articular cartilage lesion in knee (from [5]).

If an injury is so serious that the cartilage tears all the way through the thickness, e.g. a fullthickness lesion, surgery is usually recommended. Such an operation is challenging, and many factors are to be considered in order to achieve appropriate repair and rehabilitation.

One such replacement material is a condensed collagen gel that exhibits similar mechanical properties to those of native healthy articular cartilage, Fig. 1.2 .

If an acellular condensed collagen gel plug (condensed collagen gel without cells) is implanted into the human knee, then chondrocytes (cells) migrate into the plug and proliferate, which leads to wound healing. The artificial cartilage thus becomes integrated into the original one. 

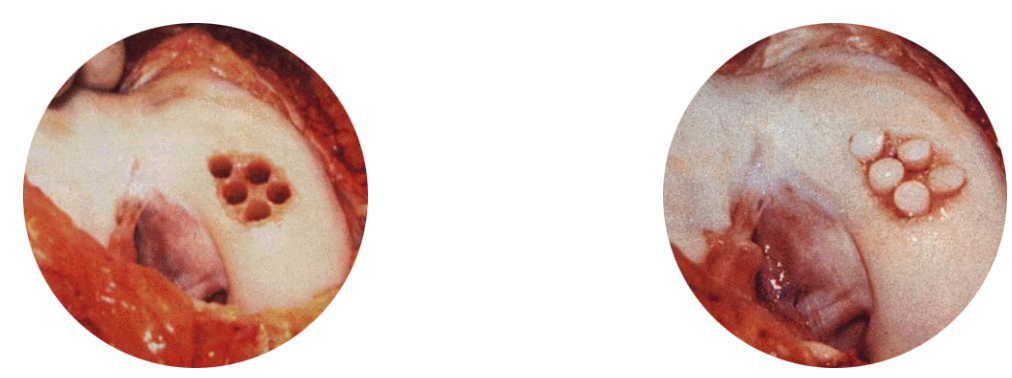

Figure 1.2: Punching and filling of articular cartilage (from [6]).

A new way to prepare the plug for implantation involves using the patient's chondrocytes (cells). If the patient is young (20 to 50 years old) and the lesion not so large (less than $4 \mathrm{~cm}$ ), a few cells are taken from the healthy side of the patient's knee cartilage and cultivated in a laboratory for several weeks. A cellular condensed collagen gel plug (newly synthesized cartilage) containing these cells is then implanted into the patient. Then, due to the activity of the cells, which attach themselves to the surrounding cartilage, the healing process of the cellular condensed collagen gel becomes faster than that of acellular condensed collagen gel.

In both cases, cells play an important role. We want to understand the material remodeling effect resulting from the cell activity. With such insight, the replacement material could be optimized through biomechanical treatment before its implantation.

The aim of the present work is to investigate the modified material properties of the cellular condensed collagen gel due to the mechanical stimulation in the framework of continuum mechanics. Especially, the modification of Young's modulus owing to the collagen type II that are synthesized by cells is the main interest.

For the experimental study, cellular condensed collagen gel specimens are incubated and stimulated mechanically in bioreactors. To follow the Young's modulus modification regarded as remodeling, a compression test is performed every week. A constitutive equation is proposed for the remodeling effect, and a remodeling velocity parameter is identified. 


\subsection{Recent work}

\section{Papers on soft tissue}

Baaijens et al. [9] reconstituted the hammock-shaped collagen architecture in heart valves and a helical pattern in arteries under the assumption that the architecture is regulated by mechanical stimulation. They developed computational models to predict the mechanoregulation for the collagen architecture and reviewed the key modeling assumptions and results achieved until 2010 .

Ricken et al. [70] proposed a triphasic model composed of solid, liquid, and nutrition for transversely isotropic saturated tissues. The growth is assumed to be governed by the stress state and the local proportion of nutrients.

Ricken and Bluhm [68] deal with the development of a multiphase model to describe the growth and remodeling phenomenon in a biological system focusing on biological optimization mechanisms. A continuum triphasic model (i.e., a solid whose interstitial spaces are filled with water containing nutrients) based on the multiphase Theory of Porous Media (TPM) is proposed to provide a thermodynamically consistent description for the growth and remodeling phenomenon. Ricken and Bluhm discuss constitutive modeling of stress-strain- or nutrient-driven growth and remodeling phenomena postulating the mass exchange of the solid phase with the nutrient phase as a function of the total Kirchhoff solid stresses, the solid Jacobian (the third invariant of right Cauchy-Green tensor related to solid), and the nutrient content.

Loret and Simões [53] assumed an internal entropy production to be quadratic in terms of the dissipation variables in order to derive a growth rate law driven by stress within the frame of a single solid in a system that is open to changes in mass only. The growth rate law is based on the concept of a convex homeostatic surface in stress space. The dissipation inequality is used as the central tool motivating the rate laws for the growth transformation and for the structural variables that define an evolving homeostatic surface. The adaptation of the tissue due to loads corresponding to non-homeostatic states is explained as a reason for finite growth.

Menzel and Waffenschmidt [56] studied growth and remodeling reflecting the alignment of 
fibrous soft biological tissue with respect to representative loading directions and used a continuum approach within a sound micro-mechanically motivated formulation. A worm-like chain model was used to describe the behavior of long-chain molecules such as those found in collagenous tissues. With the aid of a microsphere formulation, a one-dimensional constitutive equation was extended to the three-dimensional macroscopic level. With unit vectors considered for the numerical integration over the domain of the unit sphere, the anisotropic material properties were analyzed.

A constitutive model for predicting the time-dependent response of growing aneurysms during their early development was proposed by Vena et al. [82] with particular attention to cerebral aneurysms. Two mechanisms, viz., a change in the reference length of collagen fibers and a change in the collagen fiber density, are regarded as the main reason for the adaptive process. The elastic behavior is extended to the growth regime by means of a suitably developed constitutive framework based on the multiplicative decomposition of the deformation gradient into elastic and inelastic components defined for the fibers. Rate equations governing the time evolution of the fiber reference length and of the fiber density are suggested. As a result it is concluded that the two mechanisms, the change in fiber reference length and the fiber density change, have opposite effects on the stability of the tissue growth. According to Vena et al. unstable growth is obtained even for a small perturbation of the reference state for cases in which the mechanism affecting the fiber density is not sufficiently strong. They identified the constitutive parameters for the passive behavior of cerebral arterial walls by fitting experimental results taken from the literature.

Kroon [45] has investigated the remodeling effect of soft tissues accomplished by fiber-producing cells. He proposed a theoretical model for collagenous tissue and collagen gel assuming that the soft tissue is hyperelastic and the strain energy function includes a density function. This function describes the distribution of the collagenous fiber orientation and evolves according to an evolution law, according to which fibers reorient towards the direction of maximum Cauchy stress. The proposed model was able to predict both the resulting collagen distribution and stress-strain relationships of remodeled collagen gel. Kroon's theory was applied to experiments, where gels were exposed to remodeling under uniaxial and biaxial constraints. 
Kroon and Holzapfel [46] developed a constitutive representation of multiple collagen layers by taking an anisotropic strain energy function into account.

Bellouquid and Angelis [13] showed how the macroscopic tissue behavior can be described from the underlying cellular description and that this specific biological state modifies the structures of the models obtained by different assumptions using asymptotic analysis. They derived hyperbolic models and focused on the existence of global equilibrium solutions.

Muñoz, Conte, and Miodownik [80] derived a set of equilibrium equations for the stresscontrolled shape change of cells due to the remodeling and growth of their internal architecture. To separate growth processes from hyperelastic and mass-preserving deformations, they divided the deformation gradient into active and passive components. The results were applied to a truss system whose function is similar to the cytoskeletal network.

Dorfmann, Woods, and Trimmer [19] presented experimental data from cyclic loading tests of an unstimulated muscle with constant maximum stretch and different, constant engineering strain rates, and derived a constitutive model on the basis of the data.

Volokh [84] used a simple phenomenological theory to examine morphogenesis such as surface pattern emergence during the growth of anisotropic tissues. He showed that constrained growth can lead to tissue compression resulting in surface buckling.

Ehret and Itskov [21] proposed a thermodynamically consistent dissipative model describing softening phenomena in anisotropic materials based on a generalized polyconvex anisotropic strain energy function. Anisotropic softening is considered by the evolution of internal variables governing the anisotropic properties. 


\section{Papers on articular cartilage}

Mak et al. [55] obtained a mathematical solution for the indentation creep and stress-relaxation behavior of articular cartilage. They modeled articular cartilage as a homogeneous binary mixture of an incompressible, isotropic, linearly elastic solid and an incompressible, inviscid fluid.

Mow et al. [59] reviewed previous work in order to unify the understanding of the viscoelastic properties of cartilage in compression. The role of compression-dependent permeability in controlling interstitial fluid flow and its contribution to the observed viscoelastic effects are discussed. Mow et al. then use the flow-dependent viscoelastic effects to model the compressive viscoelastic properties of articular cartilage.

Ateshian and Wang [8] showed that interstitial fluid plays an important role in the load support mechanism of articular cartilage, and found that the amount of fluid decreases with time if the applied load is maintained constant. They used the linear biphasic model of Mow et al. [59] to describe the mechanical response of articular cartilage.

Federico et al. [23] developed a model of elastic properties of articular cartilage based on its microstructure. Mathematical tools developed for transversely isotropic composite materials were used to describe articular cartilage whose elastic properties are assumed to be a function of cartilage depth. Their results demonstrated that the axial elastic modulus decreases from the deep zone to the articular surface and were in good agreement with experimental findings.

Wilson et al. [85] proposed that the local stresses and strains in the collagen fibrils cannot be reliably determined without taking the local arcade-like collagen-fibril structure into account. They used a poroviscoelastic fibril-reinforced FEA model and determined the fibril properties by fitting numerical data to experimental results of unconfined compression and indentation tests.

García and Cortés [29] proposed a nonlinear biphasic viscohyperelastic model combining the intrinsic viscous effect of the proteoglycan matrix with a nonlinear hyperelastic constitutive equation. For uniaxial loading their equation reduces to a solid type viscous model in which the spring is represented by the hyperelastic function and may be used to represent the me- 
chanical behavior of the proteoglycan matrix in a fiber reinforced model of articular cartilage.

Subsequently, they [28] proposed a biphasic viscohyperelastic fibril-reinforced model that describes the intrinsic viscoelasticity of the fibrillar and nonfibrillar components of the solid phase, the nonlinear tension-compression response, and the nonlinear stress-strain curves under tension and compression. They showed that the inclusion of viscous effects in the matrix reproduces experimental data incorporating material properties for the fibers consistent with experimental tensile tests.

Kwan et al. [48] determined the material coefficients of human and bovine articular cartilage under large strain and large load conditions at equilibrium using a finite deformation biphasic theory based on the choice of a specific Helmholtz free-energy function, which satisfies the general Coleman-Noll condition and the Baker-Erickson (B-E) inequalities.

Holmes and Mow modeled [33] the behavior of the soft porous permeable solid matrix using a finite deformation biphasic theory including a Helmholtz free-energy function. They deduced the dependence of the porosity and the solidity on deformation and proposed a generalization of the exponential strain-dependent functional form for the proposed permeability.

Ehlers and Markert [20] combined a descriptive representation of the linear viscoelasticity law for the organic solid matrix with an efficient numerical treatment of the strongly coupled solidfluid problem. They considered deformation-dependent permeability effects as well.

Wu et al. [86] evaluated the commercial finite element software ABAQUS for analysing biphasic soft tissues. They compared the ABAQUS solution with the analytical solution of Mak et al. [55] and showed a good agreement.

Spilker et al. [74] accounted for the highly fibrous nature of the meniscal tissue such as articular cartilage that exhibits viscoelastic behavior under applied load. They assumed a fiberreinforced, transversely isotropic solid phase.

Ün and Spilker [81] simulated cartilage contact in diarthrodial joints. They derived timedependent contact boundary conditions using kinematic, kinetic, and geometric data from experiments. The nonlinearity associated with contact is approximated using a penetration-based method, thus reducing the nonlinear problem to two linear ones. 


\subsection{Procedure}

The present work is divided into five parts.

In Chapter 2, the following aspects of articular cartilage will be explained from a medical viewpoint: kinds, structure, mechanical properties, injury, and surgery.

To describe general biological phenomena such as growth or remodeling, the change of mass should be considered at first. It influences not only the mass balance law but also other balance laws. Therefore, general kinematics and balance laws will be derived in Chapter 3, which can be applied to any biological or non-biological system with mass change. Especially, multiple phases is assumed to take mass transition into account (multiphasic open systems). At the end of the chapter, balance laws of a closed system are derived by regarding a closed system as a special case of a open system.

In Chapter 4, several kinds of constitutive equations are introduced by classifying them according to material behaviors. Section 4.1 deals with the mechanical reactions of soft tissues in a short time-scale (in minutes), how a soft tissue reacts on external loading.

In a long time-scale (in weeks), mechanical properties of tissue can change and constitutive equations of such a change are discussed in Section 4.2. At the end of the chapter, a theoretical model is proposed to predict material behaviors in a short and a long time-scale experiment.

Chapter 5 is dedicated to experiments, which features descriptions of how to produce cellseeded soft tissue, how to stimulate the cell-seeded tissue in bioreactors, and how to perform compression tests to measure the stiffness changes of the cellular tissue every week. From every week compression test of the specimens mechanically stimulated in the bioreactors, the modification of stiffness can be followed.

The associated numerical model and results will be described in Chapter 6 .

We close the work with Chapter 7 summarizing the research and discussing some points to be considered in the future. 


\section{Chapter 2}

\section{Articular cartilage from a medical viewpoint}

This short introduction mainly follows Fox et al. [24], Mow and Ratcliffe [60], [3] and [2].

\subsection{Kinds of cartilage}

Cartilage is a connective tissue composed of specialized cells called chondroblasts that produce a large amount of extracellular matrix composed of type II collagen (except fibrocartilage which also contains type I collagen) fibers, an abundant ground substance rich in proteoglycan, and elastin fibers. Chondroblasts that get caught in the matrix are called chondrocytes in cartilage and osteocytes in bone and lie in spaces called lacunae with up to eight chondrocytes per lacuna. Cartilage is classified in three types, elastic cartilage, hyaline cartilage, and fibrocartilage.

Elastic cartilage, also named yellow cartilage, contains elastic fiber networks and collagen fibers and is found in the outer ear, larynx (voice box), and epiglottis (the entrance of the larynx). Fibrocartilage is a mixture of white fibrous tissue and cartilaginous tissue in various proportions. The flexibility and toughness of fibrocartilage depend on the fibrous tissue, while its elasticity is determined by the cartilaginous tissue. It is present, for example, in vertebral discs of the spinal cord, the pubic symphysis, bone menisci, and the temporomandibular joint. If cartilage is damaged, the body will form a scar using fibrocartilage that is tough, dense, and fibrous. The torn part is filled with the fibrocartilage, which acts as a repair mechanism. 
Articular cartilage, also referred to as hyaline cartilage, is a soft tissue which covers joint surfaces of bones and thus allows them to glide over one another with little friction. The human body contains this kind of cartilage in many areas, e.g., finger, elbow, foot, knee, spine, and rib cage. Like a cushion, cartilage distributes the load over the surface of joints. It serves also as a shock absorber. Since cartilage is mainly composed of water and collagen, shock can be absorbed by the fluid in the tissue. Proper function is enabled by the arrangement of internal components and composition.

\section{$2.2 \quad$ Structure of articular cartilage}

Diarthrodial joints allow free motion and are held together by a joint capsule, such as in the temporomandibular joint, knee, and shoulder. Articular cartilage can be mostly found in the joints and forms the growth plate by which long bones grow.

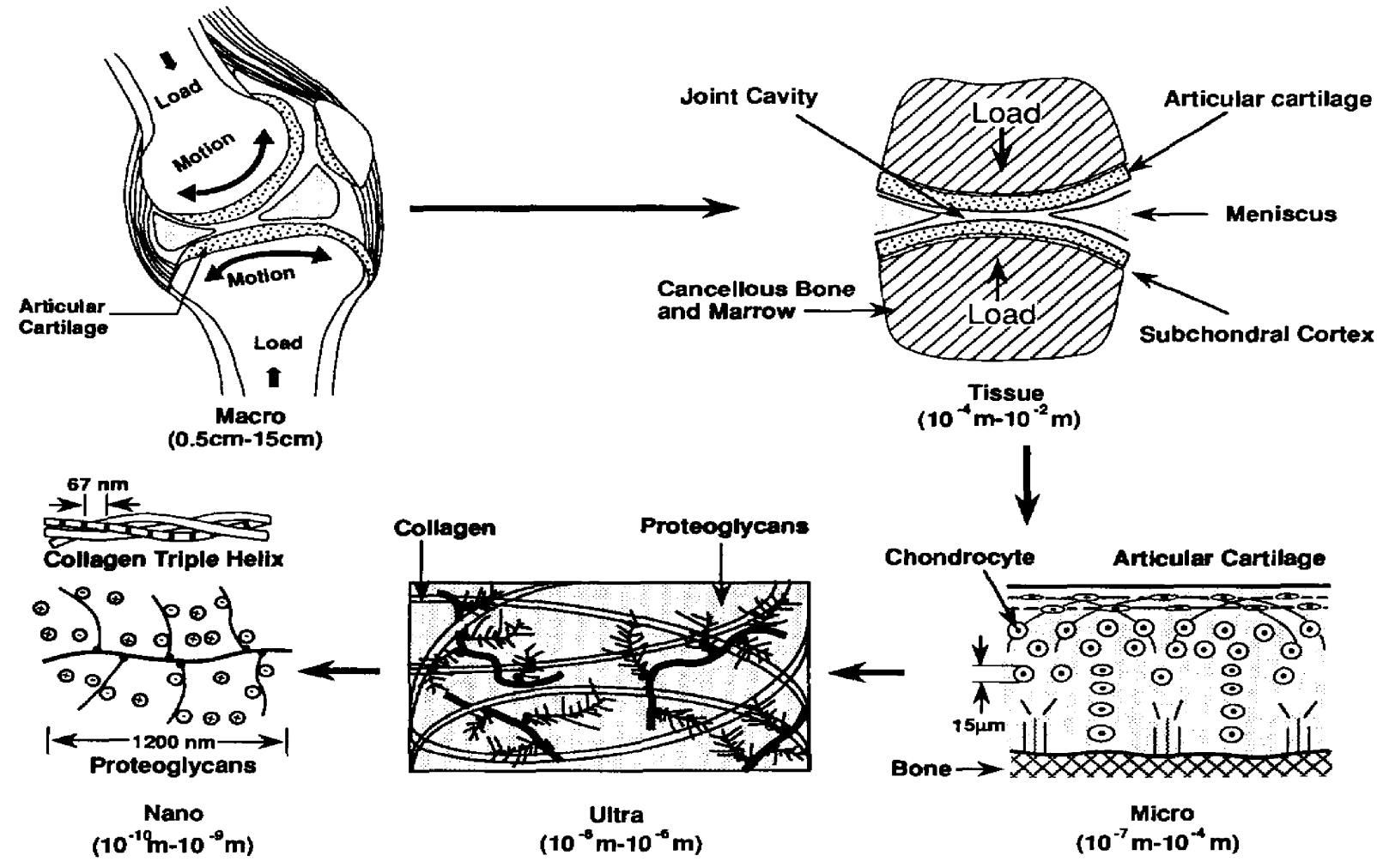

Figure 2.1: Structure of diarthrodial knee joint at different scales I (from [60]).

In Fig. 2.1, the structure of a diarthrodial knee joint at different scales is shown. 
The first diagram is at the macroscopic level (between $0.5 \mathrm{~cm}$ and $15 \mathrm{~cm}$ ), and depicts how the femur and tibia move when subjected to a bodyweight load. The top left picture in Fig. 2.1 and $\mathbf{A}$ of Fig. 2.2 show the composition of a diarthrodial joint, which consists of bone, articular cartilage, ligaments, tendons, muscle, and the joint capsule.

The articular cartilage in a diarthrodial joint is pictured at the next scale (between $10^{-4} \mathrm{~m}$ and $10^{-2} \mathrm{~m}$ ) at the top right of Fig. 2.1 which depicts the bearing surface of the joint, where the articular cartilage may look like a homogeneous solid layer.

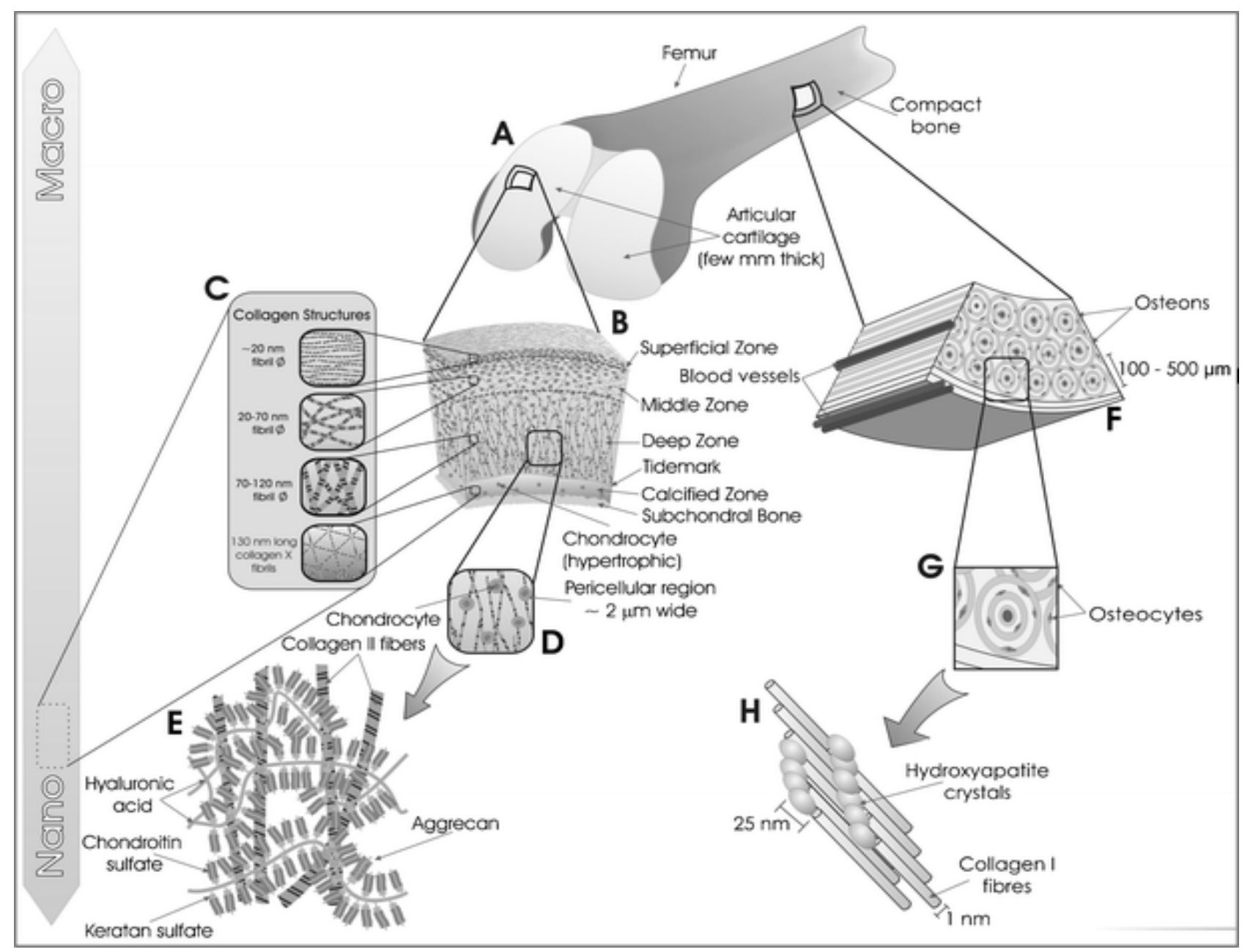

Figure 2.2: Structure of diarthrodial joint at different scales II (from [62]). 
Zooming in to between $0.1 \mu \mathrm{m}$ and $100 \mu \mathrm{m}$ (Fig. 2.1 bottom right and $\mathbf{B}$ of Fig. 2.2) microstructures such as the chondrocytes (cells that make cartilage matrix) and the organization of the collagen type II fibrils can be seen.

At the ultrastructural level (between $0.01 \mu \mathrm{m}$ and $1 \mu \mathrm{m}$ ), the individual collagen fibrils and the proteoglycan matrix can be seen (bottom middle figure of Fig. 2.1 and D of Fig. 2.2).

The interior structure of the collagen and proteoglycan appear at the nanostructural scale (bottom left figure of Fig. 2.1 and $\mathbf{E}$ of Fig. 2.2); this is not of our interest in the present work.

The structure at the micro scale (between $0.1 \mu \mathrm{m}$ and $100 \mu \mathrm{m}$ ) can be divided into four zones:

1. the superficial, tangential zone $(10-20 \%$ of the cartilage thickness),

2. the middle zone, $(40-60 \%$ of the cartilage thickness $)$,

3. the deep zone, $(30-40 \%$ of the cartilage thickness $)$, and

4. the calcified cartilage zone under which subchondral bone lies.

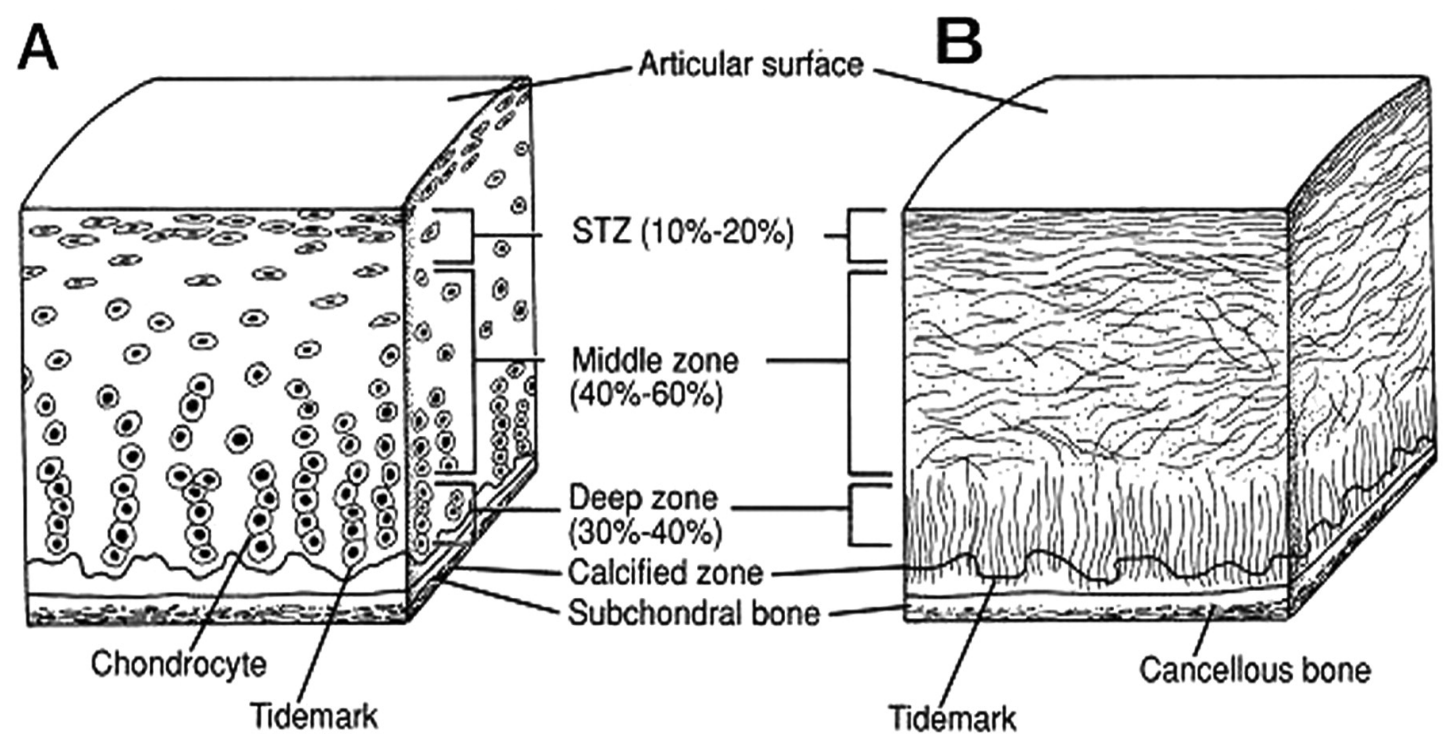

Figure 2.3: Structure of articular cartilage I (from [24]).

Depending on the zone, the organization of collagen as well as amounts of proteoglycans (an important component of the extracellular matrix) are different. A schematic of these zones is 
illustrated in Fig. 2.3. A of Fig. 2.3 illustrates the distribution of chondrocytes (cells) depending on depth, and $\mathbf{B}$ shows the orientation and density of collagen fibers.

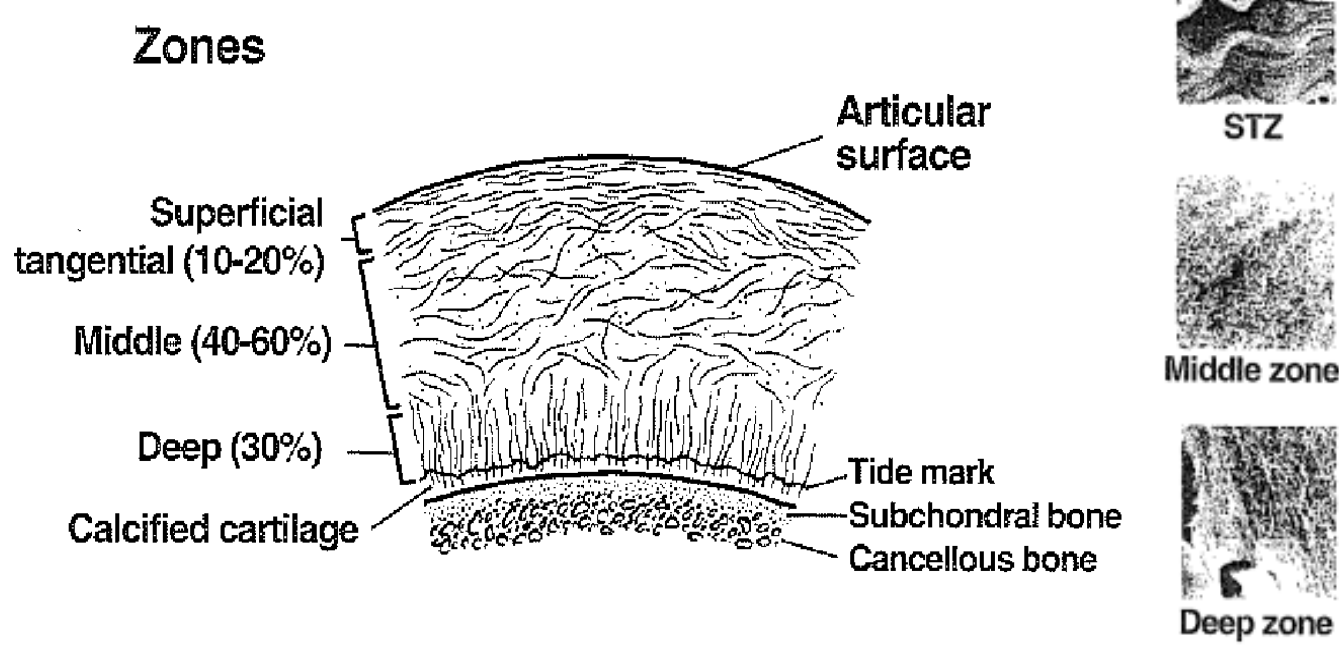

Figure 2.4: Structure of articular cartilage II (from [24]).

The superficial tangential zone contains the highest collagen content (ca. $85 \%$ by dry weight), and the direction of collagen fibrils is parallel to the joint surface. From a mechanical point of view, this zone may resist shear stresses. Moving closer to the tide mark above the subchondral bone, the amount of collagen decreases.

In this study the model is investigated at the scale of the tissue (between $10^{-1} \mathrm{~mm}$ and $10 \mathrm{~mm}$, as top right picture of Fig. 2.1). However, the synthesis of collagen type II fibrils is a microscopic phenomenon, as chondrocytes synthesize a bundle of collagen type II that is about $100 \mu \mathrm{m}$. 


\subsection{Mechanical properties of articular cartilage}

Articular cartilage is composed of two major phases

- a fluid phase containing water $(68-85 \%)$ and electrolytes, and

- a solid phase containing collagen type II (10 - 20\%), proteoglycans $(5-10 \%)$, glycoproteins, and chondrocytes that produce cartilage matrix.

Thus, the three major components of articular cartilage are essentially water, collagen type II, and proteoglycans, where mutual action decides its mechanical behavior. Changes in the relative amounts of these components due to, e.g., disease or remodeling will change the mechanical properties of cartilage. In the present work, the change of stiffness due to the synthesis of collagen type II is the main interest and addressed in Sec. 5.2.2.

Of the three major components, water is the dominant. Approximately $30 \%$ of the total water exists within the intrafibrillar space of collagen. The swelling pressure due to the fixed charge density (FCD) of the proteoglycans determines the collagen fibril diameter and the amount of water within the collagen. The proteoglycans are constrained within the collagen matrix. If an articular cartilage is compressed, fluid is partially squeezed out of the cartilage, which leads to drag forces between the fluid and solid matrix. The forces increase in proportion to increasing compression and it becomes more and more difficult to exude water. Such a phenomenon will be explained in the present work with a concept of diffusion in Section 4.3.4.

While the saturated constituents possess compressional strength, the collagen type II fiber has the tensile strength like the steel of reinforced concrete. Proteoglycan, the third major component, is a large biomolecule consisting of a protein core with glycosaminoglycan side chains. This molecule normally occupies a large space when not compacted by a collagen network. The compaction of the proteoglycans affects swelling pressure as well as fluid motion under compression. The role of proteoglycan is not considered herein.

\subsection{Injury of articular cartilage}

Traumatic mechanical destruction, direct blows, or progressive mechanical degeneration (wear and tear) results in articular cartilage injuries. Depending on the extent of the damage and 
the location of the injury, it is sometimes possible for the articular cartilage cells to heal. Since articular cartilage is not supplied with blood directly, it cannot repair itself. If the injury penetrates the bone beneath the cartilage, the underlying bone provides some blood to the area, improving the chance of healing.

Articular cartilage fragments separated from the underlying bone may float in the joint and interfere with normal joint motion. The initial damage begins with cartilage softening then progresses to fragmentation. As the loss of the articular cartilage lining continues, the underlying bone has no protection from the normal wear and tear induced from daily motion and begins to break down, leading to osteoarthritis (OA). Osteoarthritis known as degenerative joint disease is characterized by three processes:

- In the early stages of disease, the surface of the cartilage becomes inflamed and swollen. The joint loses proteoglycan molecules and other tissues, and then begins to lose water. Fissures and pits appear in the cartilage.

- As the disease progresses and more tissue is lost, the cartilage starts harden. As a result, it becomes increasingly prone to damage from repetitive use and injury.

- Eventually, large amounts of cartilage are destroyed, leaving the ends of the bone within the joint unprotected. Bone damage follows.

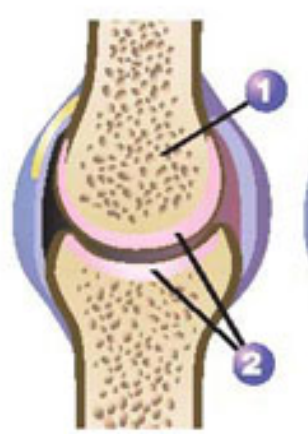

I. Bone

2. Cartilage

3. Thinning of cartilage

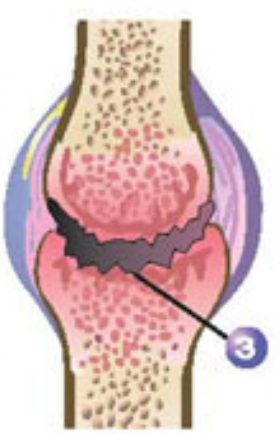

4. Cartilage remnants

5. Destruction of cartilage

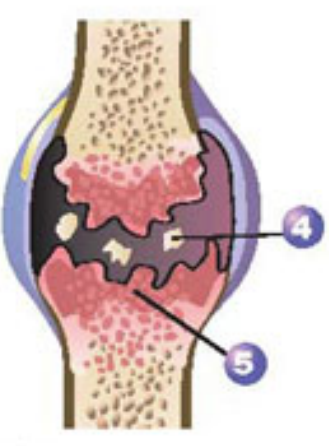

Figure 2.5: Evolution of Osteoarthritis (from [1]). 


\subsection{Reparative surgery of articular cartilage}

The type of surgical treatment to repair damaged articular cartilage depends upon numerous factors including patient age, the size and location of the lesion, and prior surgical treatments. For small lesions, Fig. 2.6, there are two methods.

The first and common practice, chondroplasty, occurs when the surgeon arthroscopically reshapes or smoothes the shredded or frayed articular cartilage, Fig. 2.6 (a). It is a simple surgical technique whereby the fibrillated (ragged) and damaged joint surface is cut, scraped, lasered or burred away in the hope that the healthy joint surface will heal over the defect.

The second and slightly more involved procedure is microfracture, where the surgeon creates several small holes in the lesion to stimulate new cartilage growth, 2.6 (b).

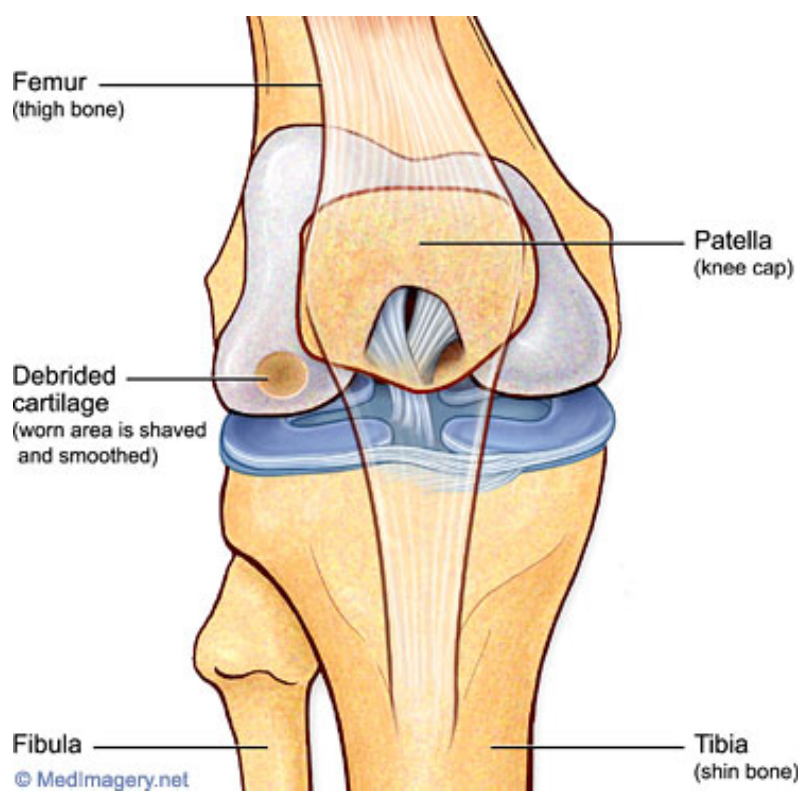

(a) Chondroplasty

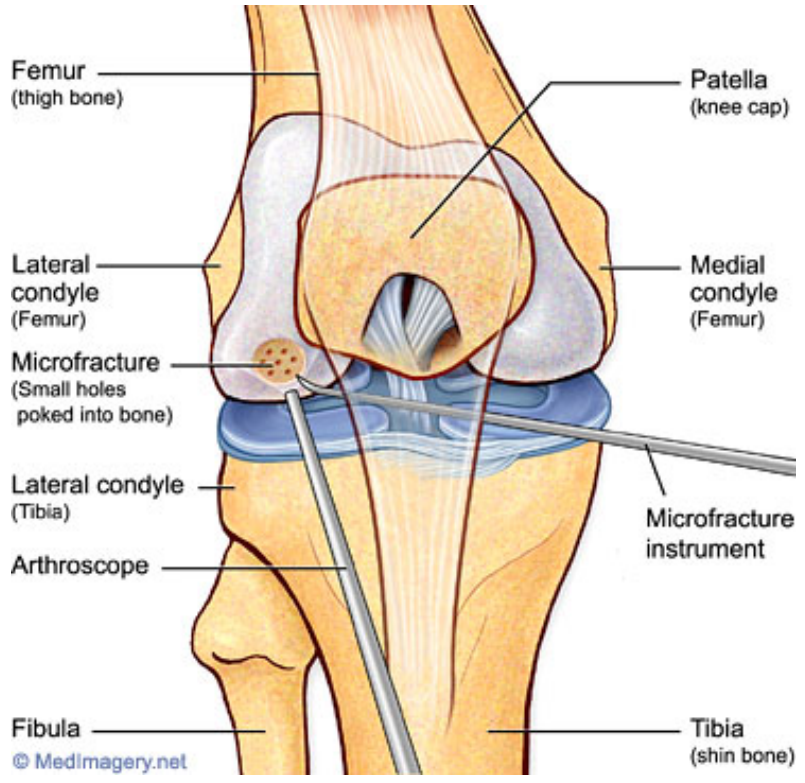

(b) Microfracture

Figure 2.6: Surgeries for small cartilage lesions (from [4]). 
In the case of large lesions (Fig. 2.7), open surgical procedures are considered, including:

- Osteochondral Autograft Resurfacing: Cartilage plugs are taken from other locations in the patient's knee and used to fill in the void at the site of injury.

- Osteochondral Allograft Resurfacing: Cartilage plugs are taken from a donor cadaver knee and are used to fill in the void at the site of injury.

- Autologous Chondrocyte Implantation: This is a two-stage procedure. In the first stage, arthroscopic surgery is performed to evaluate the chondral lesion and obtain a sample of the patient's normal articular cartilage. This sample is then sent to a laboratory where millions of cartilage cells are grown outside the body in a tissue culture over a 3-6 week period. These cells can then be implanted into the injury site during the second procedure.

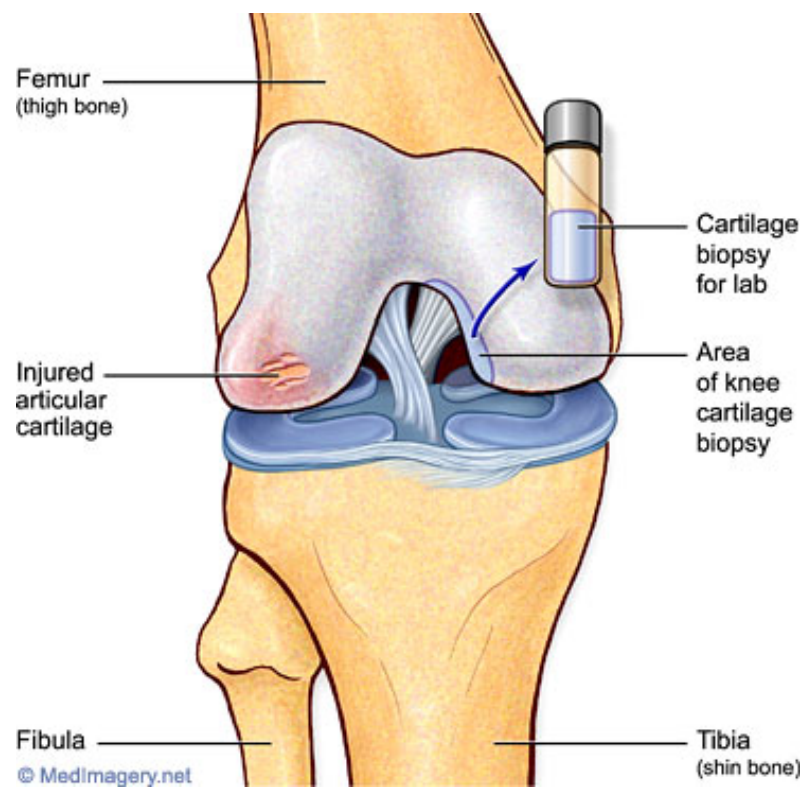

(a) Removal of a small piece of cartilage

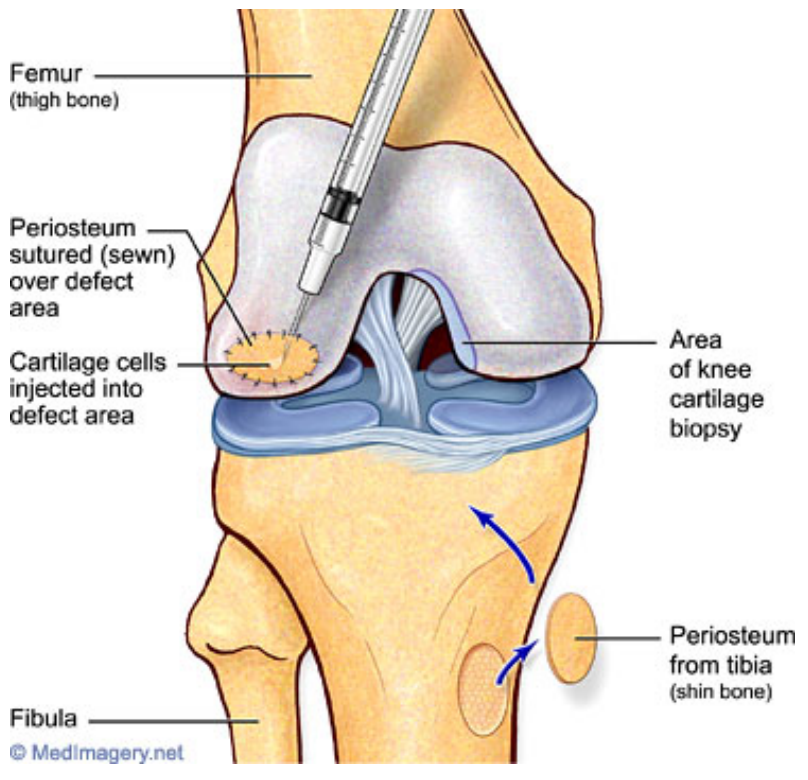

(b) Injection of cells into defect area

Figure 2.7: Autologous chondrocyte implantation for large lesions (from [4]).

This case is interesting to us, as cell activity causes remodeling of the replacement material. 


\section{Chapter 3}

\section{Kinematics and balance laws for a general biological system}

To describe biological phenomena such as growth or remodeling, the change of mass should be considered at first. The newly added mass causes additional momentum, moment of momentum, energy and entropy into the system and thus, all the balance laws should be derived on the basis of the mass balance.

\section{Monophasic model}

Kuhl and Steinmann [47] explained the influence of enhanced mass balance on the other balance equations with introducing volume-specific and mass-specific formats. According to their presentation, the mass-specific format is free from all explicit mass-dependent terms (open-system contributions) and balance equations in the format resembles those of closed-system (classical balance equations).

Lubarda and Hoger [54] presented a general constitutive theory of stress-modulated growth of biomaterials. They derived balance equations with consideration of mass source.

Epstein and Maugin [22] proposed a theory of material growth regarding growth as a local rearrangement of material inhomogeneities. Mass flux is introduced in addition to mass source in the mass balance law. In their theory all irreversible sources are lumped in extra terms and the reversible case can be identified by vanishing of the extra input. 


\section{Multiphasic model}

Cowin and Hegedus [16] developed a model for bone remodeling by regarding it as a continual process of growth, reinforcement and resorption involving. They introduced a mass source in order to describe biological growth and modified the transport theorem and balance equations. They suggested that the mass source is a function of deformation gradient and a volume fraction of matrix material in an unstrained reference state in isothermal process.

Ricken et al. [69] proposed a triphasic model for transversely isotropic saturated biological tissues including growth. They assumed that growth is determined by stress and local proportion of nutrients. Thereby, nutrients are responsible for mass exchange. They assumed that mass sources arise from inter-conversion.

Garikipati et al. [30] formulated balance equations taking mass transport into consideration for treatment of growth in biological tissue. Particularly they include multiphasic materials incorporating internal interactions between species such as interaction forces and inter-species energy transfers.

This chapter is devoted to the derivation of kinematic relations and balance laws on the basis of mass change that are applicable to any biological or nonbiological system consisting of several species. The development mainly follows the paper by Garikipati et al. [30] and extends the theory with the following points.

- Solid flux and fluid source are introduced in the present work, while Garikipati et al. did not consider these.

- The solid flux is assumed to be very slow in comparison with other fluxes.

- Entropy transfer between the species is introduced in the present work.

- In the present work a closed system is regarded as a special case of a open system and the reduction from an open system to a closed one is shown.

The interaction forces between species in their work [30] are ignored in the present work. Such forces will be required, for example, in case of electromagnetic fields but are regarded as unnecessary in biological systems. 


\subsection{Kinematics of growth}

We assume that the tissue of interest $\mathcal{B}$ occupying an open region $\Omega_{0} \subset \mathbb{R}^{3}$ in the reference configuration (at time $t=0$ ) is composed of several kinds of species symbolized by superscript $i$. Material points of the body can be described with a position vector $\boldsymbol{X}$.

Usually, soft tissues present residual stress that can be released when the tissue is excised:

$$
\boldsymbol{\sigma}(t=0) \stackrel{\text { def }}{=} \boldsymbol{\sigma}_{0}=\boldsymbol{\sigma}_{\text {res }} \neq \mathbf{0} \quad \text { in } \Omega_{0}
$$

Such a phenomenon can be observed by cutting an artery, Ogden [64]. If a short length of artery is cut radially, it springs open due to its residual stress. In this case, the residual stress is tensile stress in circumferential direction.

In order to explain the phenomenon of the residual stress, let us conceive an imaginary state $\Omega_{\text {org }}$. This is an undeformed state under stress-free condition and cannot be observed in reality,

Taber [79] and Ogden [64]. Let $d m_{\text {org }}^{i}$ and $\rho_{\text {org }}^{i}(\boldsymbol{X})$ denote differential of mass and mass density of a species $i$ in $\Omega_{\text {org }}$, respectively. Due to mass transport, the differential of mass becomes $d m_{0}^{i}$ and its density becomes $\rho_{0}^{i}(\boldsymbol{X}, t)$ in $\Omega_{0}$. If the mass density does not change $\left(\rho_{\text {org }}^{i}=\rho_{0}^{i}\right)$, the mass change means the volume change, Fig. 3.1

$$
d m_{\mathrm{org}}^{i} \quad \rightarrow \quad d m_{0}^{i} \quad \Longrightarrow \quad d V_{\mathrm{org}}^{i} \quad \rightarrow \quad d V_{0}^{i}
$$

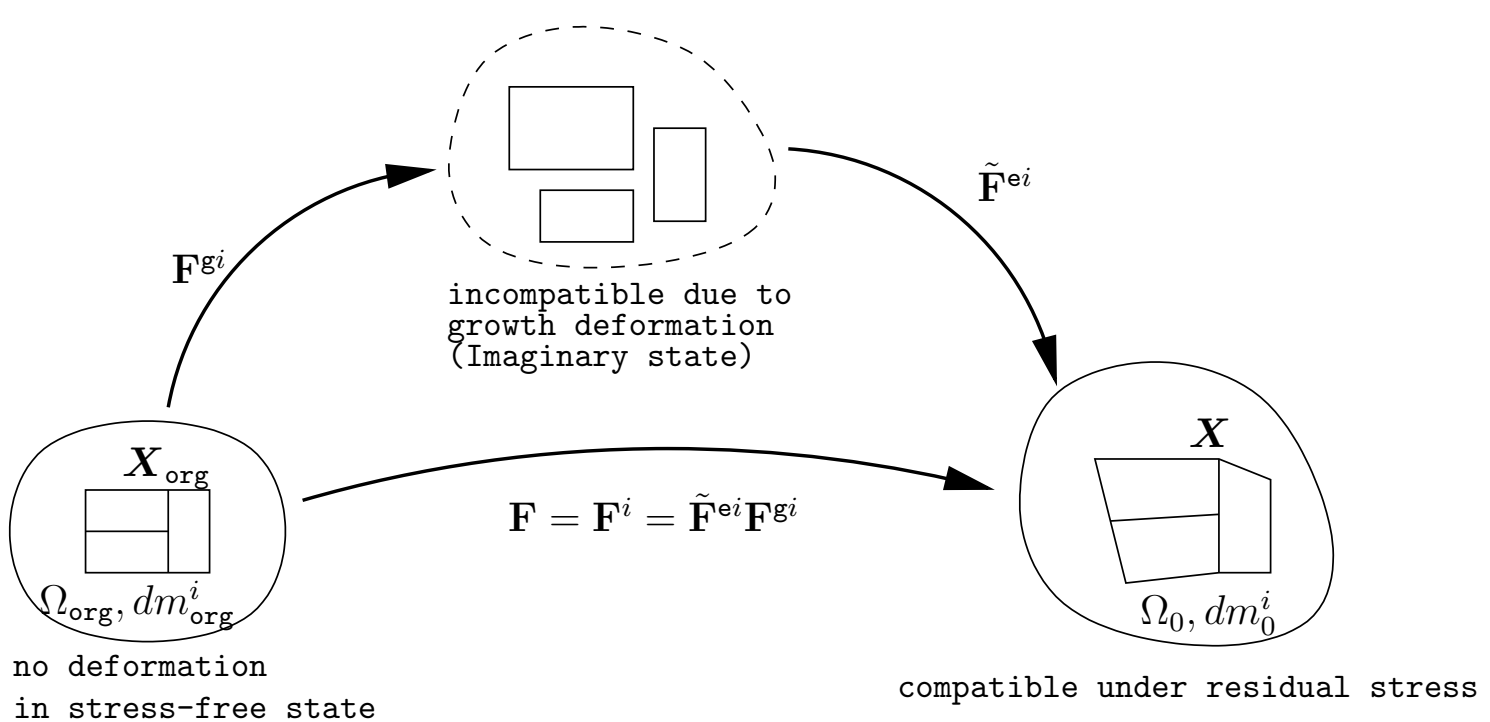

Figure 3.1: Kinematics of growing body and residual stress. 
In the case of mass influx $\left(d m_{\mathrm{org}}^{i}<d m_{0}^{i}\right)$, the species becomes lager volumetrically $\left(d V_{\mathrm{org}}^{i}<d V_{0}^{i}\right)$ and smaller $\left(d V_{\mathrm{org}}^{i}>d V_{0}^{i}\right)$ for efflux $\left(d m_{\mathrm{org}}^{i}>d m_{0}^{i}\right)$. Figure 3.2 illustrates two different cases of the residual stress. The mass of material $1 \mathrm{~m}^{1}$ changes whereas the mass of material 2 does not change.

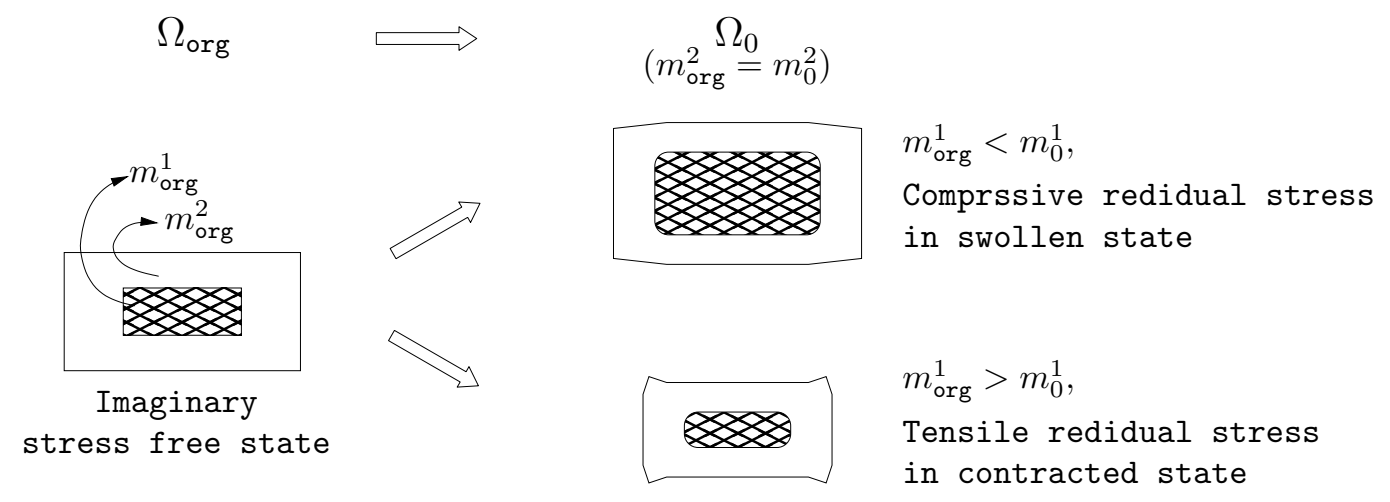

Figure 3.2: Different residual stresses.

With the assumption that the volumetric growth is isotropic, we can define the growth deformation gradient for each species:

$$
\mathbf{F}^{\mathrm{g} i}=\mathbf{F}_{\text {iso }}^{\mathrm{g} i} \stackrel{\text { def }}{=} \frac{d V_{0}^{i}}{d V_{\text {org }}^{i}} \mathbf{I}=\frac{d m_{0}^{i}}{d m_{\mathrm{org}}^{i}} \mathbf{I}=\vartheta_{\text {iso }}^{\mathrm{g} i} \mathbf{I},
$$

where $\mathbf{I}$ is the second-order identity tensor and the ratio $\vartheta^{\mathrm{g}}$ is the isotropic stretch ratio due to volumetric growth [54].

If the growth is transversely isotropic, the following growth deformation gradient can be applied

$$
\mathbf{F}^{\mathrm{g} i}=\mathbf{F}_{\mathrm{tr}}^{\mathrm{gi}} \stackrel{\text { def }}{=} \vartheta_{\mathrm{tr}}^{\mathrm{gi}} \mathbf{I}+\left(\eta_{\mathrm{tr}}^{\mathrm{gi}}-\vartheta_{\mathrm{tr}}^{\mathrm{gi}}\right) \mathbf{n}_{0} \otimes \mathbf{n}_{0}
$$

where the unit vector $\mathbf{n}_{0}$ specifies the fiber direction in reference configuration. $\eta_{\mathrm{tr}}^{\mathrm{g}}$ and $\vartheta_{\mathrm{tr}}^{\mathrm{g}}$ are the stretch ratio in the fiber direction and in any orthogonal direction to it, respectively. For orthotropic growth, we propose the following expression

$$
\mathbf{F}^{\mathrm{g} i}=\mathbf{F}_{\text {orth }}^{\mathrm{g} i} \stackrel{\text { def }}{=} \vartheta_{\text {orth }}^{\mathrm{g} i} \mathbf{I}+\left(\eta_{\text {orth }}^{\mathrm{g} i}-\vartheta_{\text {orth }}^{\mathrm{g} i}\right) \mathbf{n}_{0} \otimes \mathbf{n}_{0}+\left(\zeta_{\text {orth }}^{\mathrm{g} i}-\vartheta_{\text {orth }}^{\mathrm{g} i}\right) \mathbf{m}_{0} \otimes \mathbf{m}_{0}
$$

where the unit vectors $\mathbf{n}_{0}, \mathbf{m}_{0}$ and $\mathbf{n}_{0} \times \mathbf{m}_{0}$ specify the principal axes of orthotropy. $\eta_{\text {orth }}^{\mathrm{gi}}$ and $\zeta_{\text {orth }}^{\mathrm{g} i}$ are the stretch ratios in the direction of $\mathbf{n}_{0}$ and $\mathbf{m}_{0}$, respectively. And $\vartheta_{\text {orth }}^{\mathrm{g} i}$ is the stretch 
ratio in the direction of $\mathbf{n}_{0} \times \mathbf{m}_{0}$.

This growth deformation gradient is analogous to the plastic deformation gradient and varies pointwise. It may cause incompatibility in adjacent neighborhoods. Therefore, the tissue $\mathcal{B}$ may be no longer geometrically compatible after growth of species $i \mathbf{F}^{g i}$. For the compatibility condition, an elastic deformation $\tilde{\mathbf{F}}^{\mathrm{e} i}$ should be introduced which causes an internal stress. This is the residual stress and is different for each species, as each species has its own growth, Fig. 3.1.

$$
\mathbf{F}=\mathbf{F}^{i}=\tilde{\mathbf{F}}^{\mathrm{e} i} \mathbf{F}^{\mathrm{g} i}
$$

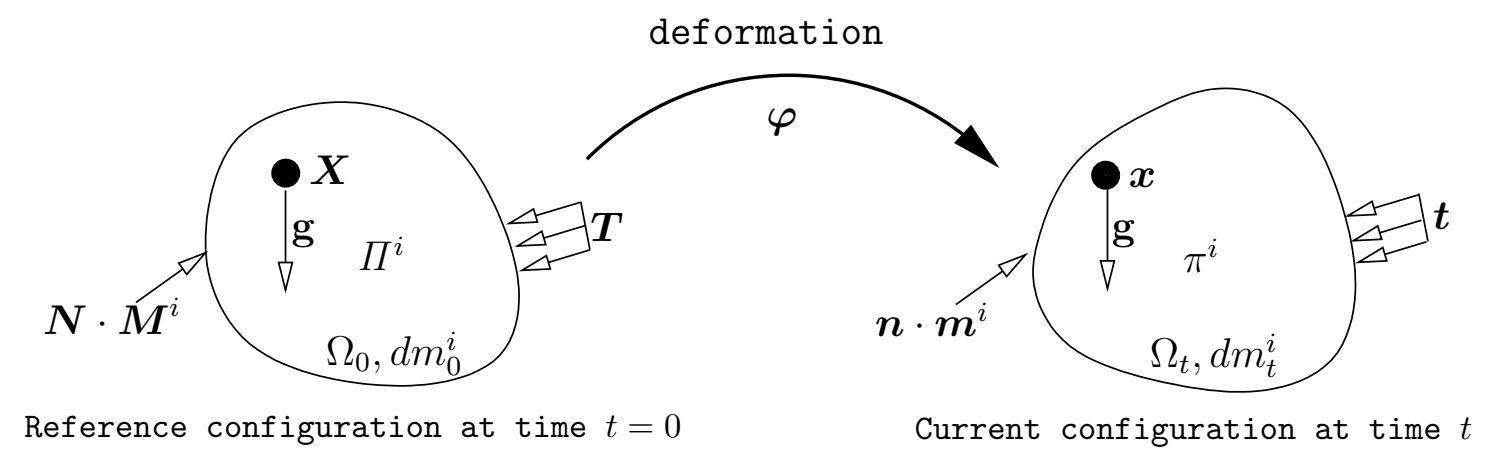

Figure 3.3: Continuum tissue with source, flux and under stress.

If external loads, such as the surface traction $\boldsymbol{T}$ or the body force $\mathbf{g}$, are applied to the body (with mass source $\Pi^{i}$ and mass flux $\boldsymbol{M}^{i}$ ) and the deformation due to the loads $\mathbf{F}^{\ell}$ is common to all species, Fig. 3.3, the total deformation gradient is

$$
\mathbf{F}=\mathbf{F}^{\ell} \mathbf{F}^{i}=\mathbf{F}^{\ell} \tilde{\mathbf{F}}^{\mathrm{e} i} \mathbf{F}^{\mathrm{g} i}
$$

If the deformation due to the external loading is elastic, $\mathbf{F}^{\ell} \tilde{\mathbf{F}}^{e i}$ denotes the total elastic deformation gradient of species $i$ :

$$
\mathbf{F}^{\ell} \tilde{\mathbf{F}}^{\mathrm{e} i} \stackrel{\text { def }}{=} \mathbf{F}^{\mathrm{e} i} \quad \Rightarrow \quad \mathbf{F}=\mathbf{F}^{\mathrm{e} i} \mathbf{F}^{\mathrm{g} i}
$$

In the current configuration $(t>0)$, the tissue body $\mathcal{B}$ occupies the new open region $\Omega_{t}$

$$
\Omega_{t}=\varphi\left(\Omega_{0}\right), \quad \Omega_{t} \subset \mathbb{R}^{3},
$$


which is a point-to-point mapping from $\Omega_{0}$ to $\Omega_{t}$.

The new position of the material points can be described with a new vector

$$
\boldsymbol{x}=\boldsymbol{\varphi}(\boldsymbol{X}, t) .
$$

The mapping of $\boldsymbol{\varphi}$ is the deformation gradient of the tissue $\mathbf{F}$ :

$$
\mathbf{F} \stackrel{\text { def }}{=} \frac{\partial \varphi}{\partial \boldsymbol{X}}
$$




\subsection{Balance laws for an open system}

\subsubsection{Balance of mass}

In classical thermodynamics, it is assumed that the material amount within a system is constant throughout any processes. Material exchange between the system and its environment is not included. Such a system is open to mechanical work and heat but is closed to mass.

Usually the biological system must be considered to be open not only to work and heat but also with respect to mass transport. The mass exchange of the system with its environment affects all the balance equations, as inflowing mass or mass newly synthesized in the system could have a certain amount of linear momentum, angular momentum, energy and entropy.

In the reference configuration, a material body $\mathcal{B}$ occupies the open region $\Omega_{0} \subset \mathbb{R}^{3}$ whose boundary is $\partial \Omega_{0}$ and its outward normal vector is $\boldsymbol{N}$. Suppose that the body is composed of solid, fluid and other species such as byproducts synthesized or excreted during a biomechanical and biochemical process and nutrient participating/consumed in the process.

Further, it is assumed that there are nothing but nutrient and one byproduct in other species. Solid and fluid are denoted by superscript S and F, respectively. Nutrition and the byproduct are denoted with superscript $\mathrm{N}$ and $\mathrm{B}$, respectively. Then the body is a multiphasic material composed of several species $(i=\mathrm{S}, \mathrm{F}, \mathrm{N}, \mathrm{B})$.

Even though the solid phase undergoes transport through solid flux, its motion $\boldsymbol{x}^{\mathrm{S}}$ can be specified entirely by the motion of the tissue material point $\boldsymbol{x}$, as the solid flux is assumed to be very slow in comparison with other fluxes.

$$
\dot{\boldsymbol{x}}^{\mathrm{S}}(\approx \dot{\boldsymbol{x}}) \ll \dot{\boldsymbol{x}}^{\mathrm{F}}, \dot{\boldsymbol{x}}^{\mathrm{N}}, \dot{\boldsymbol{x}}^{\mathrm{B}} \Rightarrow \boldsymbol{X}^{\mathrm{S}} \approx \boldsymbol{X}, \quad \boldsymbol{x}^{\mathrm{S}} \approx \boldsymbol{x} \quad \text { and } \quad \mathbf{F}^{\mathrm{S}} \approx \mathbf{F} .
$$

Thus the other flux vectors $\left(\boldsymbol{M}^{i}, i=\mathrm{F}, \mathrm{N}, \mathrm{B}\right)$ can be defined relatively to the solid phase. The fluid is described as convecting with the solid phase and diffusing with respect to it. The velocity of fluid can be expressed with respect to that of solid phase.

The total material density of the tissue is the sum of the all species densities

$$
\rho_{0}=\sum_{i} \rho_{0}^{i}(\boldsymbol{X}, t)=\rho_{0}^{\mathrm{S}}+\rho_{0}^{\mathrm{F}}+\rho_{0}^{\mathrm{N}}+\rho_{0}^{\mathrm{B}}, \quad i=\mathrm{S}, \mathrm{F}, \mathrm{N}, \mathrm{B} .
$$


The density $\rho_{0}^{i}$ can change due to mass source, mass transport through the system or interconversion of species e.g. synthesization of byproduct (such as collagen type II) by consuming nutrition (a biochemical process).

Generally speaking, we can say that the time rate of mass change of species $i$ is balanced with its volumetric mass exchange and its mass exchange due to contact mass flux, that are features of the open systems:

$$
\frac{d}{d t} m^{i}=\bar{m}_{\mathrm{open}}^{i} \stackrel{\text { def }}{=} \bar{m}_{\mathrm{source}}^{i}+\bar{m}_{\mathrm{flux}}^{i} .
$$

Thus, the mass balance for any species $(i=\mathrm{S}, \mathrm{F}, \mathrm{N}, \mathrm{B})$ can be written in integral form:

$$
\frac{d}{d t} \int_{m_{0}^{i}} d m_{0}^{i}=\frac{d}{d t} \int_{\Omega_{0}} \rho_{0}^{i} d V=\int_{\Omega_{0}} \Pi^{i} d V-\int_{\partial \Omega_{0}} \boldsymbol{M}^{i} \cdot d \boldsymbol{A},
$$

where

$$
\begin{aligned}
\bar{m}_{\text {source }} & \stackrel{\text { def }}{=} \int_{\Omega_{0}} \Pi^{i} d V, \\
\bar{m}_{\text {flux }} & \stackrel{\text { def }}{=}-\int_{\partial \Omega_{0}} \boldsymbol{M}^{i} \cdot d \boldsymbol{A},
\end{aligned}
$$

with the infinitesimal volume element $d V$ and the area element vector $d \boldsymbol{A}(\stackrel{\text { def }}{=} \boldsymbol{N} d A)$ defined in the reference configuration.

With the divergence theorem, we can obtain its differential form as

$$
\begin{array}{r}
\int_{\Omega_{0}} \frac{d}{d t} \rho_{0}^{i} d V=\int_{\Omega_{0}}\left(\Pi^{i}-\operatorname{Div} \boldsymbol{M}^{i}\right) d V, \\
\rightarrow \quad \frac{d \rho_{0}^{i}}{d t}=\Pi^{i}-\operatorname{Div} \boldsymbol{M}^{i}, \quad \forall i=\mathrm{S}, \mathrm{F}, \mathrm{N}, \mathrm{B} .
\end{array}
$$

Due to the contributions of mass source and flux, the time rate of mass change is not zero. Otherwise, the system is a closed one (no mass change rate) and Eq. (3.2.5) is reduced to the continuity equation familiar in classical continuum mechanics.

With the further assumption that the sources are generated as a result of inter-conversion, we can simplify the relation of sources as follows

$$
\sum_{i} \Pi^{i}=\Pi^{\mathrm{S}}+\Pi^{\mathrm{F}}+\Pi^{\mathrm{N}}+\Pi^{\mathrm{B}}=0
$$


Summing up over $i$ of Eq. (3.2.5) and considering Eq. (3.2.6) and (3.2.2) gives the balance of mass for the whole system:

$$
\frac{d}{d t} \rho_{0}=-\sum_{i} \operatorname{Div} \boldsymbol{M}^{i}
$$

The time rate of tissue material density depends on the mass fluxes $\boldsymbol{M}^{i}(i=\mathrm{S}, \mathrm{F}, \mathrm{N}, \mathrm{B})$.

\subsubsection{Balance of linear momentum}

The tissue of interest $\mathcal{B}$ in the reference configuration $\Omega_{0}$ is subjected to surface traction $\boldsymbol{T}$ and the body force per unit mass $\mathbf{g}$.

The natural boundary condition is

$$
\boldsymbol{T}=\sum_{i} \boldsymbol{T}^{i}=\sum_{i} \mathbf{P}^{i} \boldsymbol{N}=\mathbf{P} \boldsymbol{N} \quad \text { on } \partial \Omega_{0}, \quad i=\mathrm{S}, \mathrm{F}, \mathrm{N}, \mathrm{B} .
$$

$\boldsymbol{T}^{i}$ is the partial surface fraction of species $i, \mathbf{P}^{i}$ is the partial first Piola-Kirchhoff stress tensor of species $i$ whose summation over the species is the first Piola-Kirchhoff stress tensor of the system (tissue):

$$
\mathbf{P}=\sum_{i} \mathbf{P}^{i}, \quad i=\mathrm{S}, \mathrm{F}, \mathrm{N}, \mathrm{B}
$$

If the tissue deforms with $\varphi(\mathbf{X}, t)$, its time derivative is a material velocity of tissue and the same as that of solid phase due to the assumption in (3.2.1):

$$
\boldsymbol{V}(\boldsymbol{X}, t)=\frac{\partial \varphi}{\partial t} \approx \frac{\partial \boldsymbol{x}^{\mathrm{S}}}{\partial t}
$$

The material velocity of any species $i, \boldsymbol{V}^{i}$, can be defined as relative to the solid phase :

$$
\boldsymbol{V}^{i} \stackrel{\text { def }}{=} \frac{\mathbf{F} \boldsymbol{M}^{i}}{\rho_{0}^{i}}, \quad i=\mathrm{S}, \mathrm{F}, \mathrm{N}, \mathrm{B}
$$

The material velocity of solid phase is neglected because of Eq. (3.2.1):

$$
V^{\mathrm{S}} \approx 0
$$

The rest species $(i=\mathrm{F}, \mathrm{N}, \mathrm{B})$ except for solid deform with the solid phase and diffuse relatively to it. Thus, the deformation gradient of tissue $\mathbf{F}\left(=\mathbf{F}^{\mathbf{S}}\right)$ is common to all species. 
With the total material velocity of the solid phase defined as $\boldsymbol{V}$ the absolute material velocity of species $i$ can be described as $\boldsymbol{V}+\boldsymbol{V}^{i}(i=\mathrm{S}, \mathrm{F}, \mathrm{N}, \mathrm{B})$.

We define the linear momentum of any species $i$ as

$$
\boldsymbol{p}^{i} \stackrel{\text { def }}{=} \int_{\Omega_{0}} \rho_{0}^{i}\left(\boldsymbol{V}+\boldsymbol{V}^{i}\right) d V
$$

The rate of change of the momentum $\boldsymbol{p}^{i}$ is balanced with the momentum exchange due to surface and volume forces that are both divided into closed and open parts. As in classical continuum mechanics, the closed part $\overline{\boldsymbol{p}}_{\text {closed }}^{i}$ describes the external force exerted on the body excluding mass sources and fluxe. The mass source and the mass flux cause an additional change in the linear momentum which is denoted by $\overline{\boldsymbol{p}}_{\text {open }}^{i}$ :

$$
\frac{d}{d t} \boldsymbol{p}^{i}=\overline{\boldsymbol{p}}_{\mathrm{closed}}^{i}+\overline{\boldsymbol{p}}_{\text {open }}^{i}
$$

where we define

$$
\begin{gathered}
\overline{\boldsymbol{p}}_{\text {closed }}^{i} \stackrel{\text { def }}{=} \int_{\Omega_{0}} \rho_{0}^{i} \mathbf{g} d V+\int_{\partial \Omega_{0}} \boldsymbol{T}^{i} d A, \\
\overline{\boldsymbol{p}}_{\text {open }}^{i} \stackrel{\text { def }}{=} \overline{\boldsymbol{p}}_{\text {source }}^{i}+\overline{\boldsymbol{p}}_{\text {flux }}^{i}, \\
\overline{\boldsymbol{p}}_{\text {source }}^{i} \stackrel{\text { def }}{=} \int_{\Omega_{0}} \Pi^{i}\left(\boldsymbol{V}+\boldsymbol{V}^{i}\right) d V, \\
\overline{\boldsymbol{p}}_{\text {flux }}^{i} \stackrel{\text { def }}{=}-\int_{\partial \Omega_{0}}\left(\boldsymbol{V}+\boldsymbol{V}^{i}\right) \boldsymbol{M}^{i} \cdot \boldsymbol{N} d A .
\end{gathered}
$$

The balance of the linear momentum of species $i$ is postulated as:

$$
\begin{aligned}
\frac{d}{d t} \int_{\Omega_{0}} \rho_{0}^{i}\left(\boldsymbol{V}+\boldsymbol{V}^{i}\right) d V & =\int_{\Omega_{0}} \rho_{0}^{i} \mathbf{g} d V+\int_{\partial \Omega_{0}} \boldsymbol{T}^{i} d A \\
& +\int_{\Omega_{0}} \Pi^{i}\left(\boldsymbol{V}+\boldsymbol{V}^{i}\right) d V-\int_{\partial \Omega_{0}}\left(\boldsymbol{V}+\boldsymbol{V}^{i}\right) \boldsymbol{M}^{i} \cdot \boldsymbol{N} d A .
\end{aligned}
$$

The first term on the right-hand side is the at-a-distance force due to the body-force density $\mathbf{g}$ and the second term is the contact force. These forces can be seen in the classical continuum mechanics. An additional volume force is brought about due to the mass source $\Pi^{i}$. The mass flux $\boldsymbol{M}^{i}$ induces an additional momentum exchange due to contact, that is the last term. 
With rearrangement and considering Eq. (3.2.8), the above equation becomes

$$
\begin{aligned}
& \frac{d}{d t} \int_{\Omega_{0}} \rho_{0}^{i}\left(\boldsymbol{V}+\boldsymbol{V}^{i}\right) d V \\
& \quad=\int_{\Omega_{0}}\left[\rho_{0}^{i} \mathbf{g}+\Pi^{i}\left(\boldsymbol{V}+\boldsymbol{V}^{i}\right)\right] d V+\int_{\partial \Omega_{0}}\left[\mathbf{P}^{i}-\left(\boldsymbol{V}+\boldsymbol{V}^{i}\right) \otimes \boldsymbol{M}^{i}\right] \cdot \boldsymbol{N} d A \\
& \quad=\int_{\Omega_{0}}\left[\rho_{0}^{i} \mathbf{g}+\Pi^{i}\left(\boldsymbol{V}+\boldsymbol{V}^{i}\right)+\operatorname{Div}\left(\mathbf{P}^{i}-\left(\boldsymbol{V}+\boldsymbol{V}^{i}\right) \otimes \boldsymbol{M}^{i}\right)\right] d V .
\end{aligned}
$$

The left- and right-hand side of (3.2.17) are

$$
\begin{aligned}
\mathrm{LHS} & =\frac{d \rho_{0}^{i}}{d t}\left(\boldsymbol{V}+\boldsymbol{V}^{i}\right)+\rho_{0}^{i} \frac{d\left(\boldsymbol{V}+\boldsymbol{V}^{i}\right)}{d t}, \\
\mathrm{RHS} & =\rho_{0}^{i} \mathbf{g}+\Pi^{i}\left(\boldsymbol{V}+\boldsymbol{V}^{i}\right)+\operatorname{Div}\left[\mathbf{P}^{i}-\left(\boldsymbol{V}+\boldsymbol{V}^{i}\right) \otimes \boldsymbol{M}^{i}\right] \\
& =\rho_{0}^{i} \mathbf{g}+\rho_{0}^{i}+\Pi^{i}\left(\boldsymbol{V}+\boldsymbol{V}^{i}\right)+\operatorname{Div} \mathbf{P}^{i}-\operatorname{Div}\left[\left(\boldsymbol{V}+\boldsymbol{V}^{i}\right) \otimes \boldsymbol{M}^{i}\right] \\
& =\rho_{0}^{i} \mathbf{g}+\Pi^{i}\left(\boldsymbol{V}+\boldsymbol{V}^{i}\right)+\operatorname{Div} \mathbf{P}^{i}-\operatorname{Grad}\left(\boldsymbol{V}+\boldsymbol{V}^{i}\right) \boldsymbol{M}^{i}-\left(\boldsymbol{V}+\boldsymbol{V}^{i}\right) \operatorname{Div} \boldsymbol{M}^{i} .
\end{aligned}
$$

Considering Eq. (3.2.5), the following relation

$$
\frac{d \rho_{0}^{i}}{d t}\left(\boldsymbol{V}+\boldsymbol{V}^{i}\right)=\Pi^{i}\left(\boldsymbol{V}+\boldsymbol{V}^{i}\right)-\left(\boldsymbol{V}+\boldsymbol{V}^{i}\right) \operatorname{Div} \boldsymbol{M}^{i}
$$

is valid, and therefore Eq. (3.2.17) reads

$$
\rho_{0}^{i} \frac{d\left(\boldsymbol{V}+\boldsymbol{V}^{i}\right)}{d t}=\rho_{0}^{i} \mathbf{g}+\operatorname{Div} \mathbf{P}^{i}-\operatorname{Grad}\left(\boldsymbol{V}+\boldsymbol{V}^{i}\right) \boldsymbol{M}^{i} .
$$

Summing over $i(i=\mathrm{S}, \mathrm{F}, \mathrm{N}, \mathrm{B})$ of (3.2.17) gives the balance of linear momentum of the whole system:

$$
\begin{aligned}
\sum_{i} \frac{d}{d t} \int_{\Omega_{0}}\left[\rho_{0}^{i}\left(\boldsymbol{V}+\boldsymbol{V}^{i}\right)\right] d V= & \sum_{i} \int_{\Omega_{0}}\left[\rho_{0}^{i} \mathbf{g}+\Pi^{i}\left(\boldsymbol{V}+\boldsymbol{V}^{i}\right)\right] d V \\
& +\sum_{i} \int_{\partial \Omega_{0}}\left[\mathbf{P}^{i}-\left(\boldsymbol{V}+\boldsymbol{V}^{i}\right) \otimes \boldsymbol{M}^{i}\right] \cdot \boldsymbol{N} d A .
\end{aligned}
$$

Its left-hand side of (3.2.20) is

$$
\begin{aligned}
\text { LHS } & =\sum_{i} \frac{d}{d t} \int_{\Omega_{0}}\left[\rho_{0}^{i}\left(\boldsymbol{V}+\boldsymbol{V}^{i}\right)\right] d V=\frac{d}{d t} \int_{\Omega_{0}} \sum_{i}\left[\rho_{0}^{i}\left(\boldsymbol{V}+\boldsymbol{V}^{i}\right)\right] d V \\
& =\frac{d}{d t} \int_{\Omega_{0}}\left[\sum_{i} \rho_{0}^{i} \boldsymbol{V}+\sum_{i}\left(\rho_{0}^{i} \boldsymbol{V}^{i}\right)\right] d V \stackrel{(3.2 .2)}{=} \frac{d}{d t} \int_{\Omega_{0}}\left[\rho_{0} \boldsymbol{V}+\sum_{i}\left(\rho_{0}^{i} \boldsymbol{V}^{i}\right)\right] d V,
\end{aligned}
$$


and the right-hand side of (3.2.20) is

$$
\begin{aligned}
\text { RHS } & \sum_{i} \int_{\Omega_{0}}\left[\rho_{0}^{i} \mathbf{g}+\Pi^{i}\left(\boldsymbol{V}+\boldsymbol{V}^{i}\right)\right] d V+\sum_{i} \int_{\partial \Omega_{0}}\left[\mathbf{P}^{i}-\left(\boldsymbol{V}+\boldsymbol{V}^{i}\right) \otimes \boldsymbol{M}^{i}\right] \cdot \boldsymbol{N} d A \\
= & \int_{\Omega_{0}}\left[\sum_{i} \rho_{0}^{i} \mathbf{g}+\sum_{i} \Pi^{i}\left(\boldsymbol{V}+\boldsymbol{V}^{i}\right)\right] d V \\
& +\int_{\partial \Omega_{0}}\left[\sum_{i} \mathbf{P}^{i}-\sum_{i}\left(\boldsymbol{V}+\boldsymbol{V}^{i}\right) \otimes \boldsymbol{M}^{i}\right] \cdot \boldsymbol{N} d A \\
= & \int_{\Omega_{0}}\left[\rho_{0} \mathbf{g}+\sum_{i}\left(\Pi^{i} \boldsymbol{V}^{i}\right)\right] d V+\int_{\partial \Omega_{0}}\left[\mathbf{P}-\sum_{i}\left(\boldsymbol{V}+\boldsymbol{V}^{i}\right) \otimes \boldsymbol{M}^{i}\right] \cdot \boldsymbol{N} d A,
\end{aligned}
$$

where the term $\sum_{i}\left(\Pi^{i} \boldsymbol{V}\right)$ is set to zero using Eq. (3.2.6).

With an assumption that the linear momentum of the system depends only on external causes such as $\mathbf{g}, \mathbf{P}$ and $\boldsymbol{M}^{i}$, the internal interaction between the species $\sum_{i}\left(\Pi^{i} \boldsymbol{V}^{i}\right)$ can be cancelled out

$$
\sum_{i}\left(\Pi^{i} \boldsymbol{V}^{i}\right)=\boldsymbol{O}
$$

Then the Eq. (3.2.20) yields

$$
\begin{aligned}
\frac{d}{d t} \int_{\Omega_{0}}\left[\rho_{0} \boldsymbol{V}+\sum_{i}\left(\rho_{0}^{i} \boldsymbol{V}^{i}\right)\right] d V & =\int_{\Omega_{0}} \rho_{0} \mathbf{g} d V+\int_{\partial \Omega_{0}}\left[\mathbf{P}-\sum_{i}\left(\boldsymbol{V}+\boldsymbol{V}^{i}\right) \otimes \boldsymbol{M}^{i}\right] \cdot \boldsymbol{N} d A \\
& =\int_{\Omega_{0}}\left[\rho_{0} \mathbf{g}+\operatorname{Div}\left(\mathbf{P}-\sum_{i}\left(\boldsymbol{V}+\boldsymbol{V}^{i}\right) \otimes \boldsymbol{M}^{i}\right)\right] d V
\end{aligned}
$$

and its local form is

$$
\frac{d}{d t}\left[\rho_{0} \boldsymbol{V}+\sum_{i}\left(\rho_{0}^{i} \boldsymbol{V}^{i}\right)\right]=\rho_{0} \mathbf{g}+\operatorname{Div}\left(\mathbf{P}-\sum_{i}\left(\boldsymbol{V}+\boldsymbol{V}^{i}\right) \otimes \boldsymbol{M}^{i}\right) .
$$

The time rate of change of linear momentum of the entire system is the sum of the body force and the traction that is affected by the mass flux vector. 


\subsubsection{Balance of angular momentum}

We define the angular momentum of any species $i$ with respect to the origin of a coordinate system as

$$
\boldsymbol{l}^{i} \stackrel{\text { def }}{=} \int_{\Omega_{0}} \boldsymbol{\varphi} \times \rho_{0}^{i}\left(\boldsymbol{V}+\boldsymbol{V}^{i}\right) d V .
$$

The rate of change of the angular momentum $\boldsymbol{l}^{i}$ equals the total moment acting on the species, which consists of a closed part $\overline{\boldsymbol{l}}_{\text {closed }}^{i}$ and an open part $\overline{\boldsymbol{l}}_{\text {open }}^{i}$. As in classical continuum mechanics, the closed part describes the moment of the external force $\overline{\boldsymbol{p}}_{\text {closed }}^{i}$ defined in (3.2.15a). An open part $\bar{l}_{\text {open }}^{i}$ arises due to the moment of mass source and mass flux from (3.2.15b):

$$
\frac{d}{d t} \boldsymbol{l}^{i}=\overline{\boldsymbol{l}}_{\mathrm{closed}}^{i}+\overline{\boldsymbol{l}}_{\mathrm{open}}^{i}
$$

We define

$$
\begin{aligned}
& \overline{\boldsymbol{l}}_{\text {closed }}^{i} \stackrel{\text { def }}{=} \boldsymbol{\varphi} \times \overline{\boldsymbol{p}}_{\text {closed }}^{i}=\int_{\Omega_{0}} \boldsymbol{\varphi} \times \rho_{0}^{i} \mathbf{g} d V+\int_{\partial \Omega_{0}} \boldsymbol{\varphi} \times \mathbf{P}^{i} \cdot \boldsymbol{N} d A, \\
& \overline{\boldsymbol{l}}_{\text {open }}^{i} \stackrel{\text { def }}{=} \boldsymbol{\varphi} \times \overline{\boldsymbol{p}}_{\text {open }}^{i}=\overline{\boldsymbol{l}}_{\text {source }}^{i}+\overline{\boldsymbol{l}}_{\text {flux }}^{i}, \\
& \overline{\boldsymbol{l}}_{\text {source }}^{i} \stackrel{\text { def }}{=} \boldsymbol{\varphi} \times \overline{\boldsymbol{p}}_{\text {source }}^{i}=\int_{\Omega_{0}} \boldsymbol{\varphi} \times \Pi^{i}\left(\boldsymbol{V}+\boldsymbol{V}^{i}\right) d V, \\
& \overline{\boldsymbol{l}}_{\text {flux }}^{i} \stackrel{\text { def }}{=} \boldsymbol{\varphi} \times \overline{\boldsymbol{p}}_{\text {flux }}^{i}=-\int_{\partial \Omega_{0}} \boldsymbol{\varphi} \times\left(\boldsymbol{V}+\boldsymbol{V}^{i}\right) \otimes \boldsymbol{M}^{i} \cdot \boldsymbol{N} d A .
\end{aligned}
$$

The balance of angular momentum of species $i$ is postulated as:

$$
\begin{aligned}
\frac{d}{d t} \int_{\Omega_{0}} \boldsymbol{\varphi} \times \rho_{0}^{i}\left(\boldsymbol{V}+\boldsymbol{V}^{i}\right) d V= & \int_{\Omega_{0}} \boldsymbol{\varphi} \times\left[\rho_{0}^{i} \mathbf{g}+\Pi^{i}\left(\boldsymbol{V}+\boldsymbol{V}^{i}\right)\right] d V \\
& +\int_{\partial \Omega_{0}} \boldsymbol{\varphi} \times\left[\mathbf{P}^{i}-\left(\boldsymbol{V}+\boldsymbol{V}^{i}\right) \otimes \boldsymbol{M}^{i}\right] \cdot \boldsymbol{N} d A .
\end{aligned}
$$

The same can be obtained from (3.2.17) multiplied by position vector $\boldsymbol{\varphi}$.

The left-hand side of (3.2.27) is

$$
\begin{aligned}
\text { LHS } & =\frac{d}{d t} \int_{\Omega_{0}}\left[\boldsymbol{\varphi} \times \rho_{0}^{i}\left(\boldsymbol{V}+\boldsymbol{V}^{i}\right)\right] d V \\
& =\int_{\Omega_{0}}\left\{\frac{d \boldsymbol{\varphi}}{d t} \times \rho_{0}^{i}\left(\boldsymbol{V}+\boldsymbol{V}^{i}\right)+\boldsymbol{\varphi} \times\left[\frac{d \rho_{0}^{i}}{d t}\left(\boldsymbol{V}+\boldsymbol{V}^{i}\right)+\rho_{0}^{i} \frac{d\left(\boldsymbol{V}+\boldsymbol{V}^{i}\right)}{d t}\right]\right\} d V \\
& =\int_{\Omega_{0}}\left\{\boldsymbol{V} \times \rho_{0}^{i} \boldsymbol{V}^{i}+\boldsymbol{\varphi} \times\left[\frac{d \rho_{0}^{i}}{d t}\left(\boldsymbol{V}+\boldsymbol{V}^{i}\right)+\rho_{0}^{i} \frac{d\left(\boldsymbol{V}+\boldsymbol{V}^{i}\right)}{d t}\right]\right\} d V,
\end{aligned}
$$


and the right-hand side is

RHS

$$
\begin{aligned}
& =\int_{\Omega_{0}} \boldsymbol{\varphi} \times\left[\rho_{0}^{i} \mathbf{g}+\Pi^{i}\left(\boldsymbol{V}+\boldsymbol{V}^{i}\right)\right] d V+\int_{\partial \Omega_{0}} \boldsymbol{\varphi} \times\left[\mathbf{P}^{i}-\left(\boldsymbol{V}+\boldsymbol{V}^{i}\right) \otimes \boldsymbol{M}^{i}\right] \cdot \boldsymbol{N} d A \\
& \stackrel{(\mathrm{A.4.1)})}{=} \int_{\Omega_{0}} \boldsymbol{\varphi} \times\left[\rho_{0}^{i} \mathbf{g}+\Pi^{i}\left(\boldsymbol{V}+\boldsymbol{V}^{i}\right)\right] d V \\
& \quad+\int_{\Omega_{0}}\left\{\boldsymbol{\varphi} \times \operatorname{Div}\left[\mathbf{P}^{i}-\left(\boldsymbol{V}+\boldsymbol{V}^{i}\right) \otimes \boldsymbol{M}^{i}\right]+\mathcal{E}: \operatorname{Grad} \boldsymbol{\varphi}\left[\mathbf{P}^{i}-\left(\boldsymbol{V}+\boldsymbol{V}^{i}\right) \otimes \boldsymbol{M}^{i}\right]^{\mathrm{T}}\right\} d V .
\end{aligned}
$$

By virtue of the identity (A.4.7), we can rewrite the term with divergence operator

$\operatorname{Div}\left[\mathbf{P}^{i}-\left(\boldsymbol{V}+\boldsymbol{V}^{i}\right) \otimes \boldsymbol{M}^{i}\right]=\operatorname{Div} \mathbf{P}^{i}-\operatorname{Grad}\left(\boldsymbol{V}+\boldsymbol{V}^{i}\right) \boldsymbol{M}^{i}-\left(\boldsymbol{V}+\boldsymbol{V}^{i}\right) \operatorname{Div} \boldsymbol{M}^{i}$

By equating RHS and LHS of (3.2.27), we obtain

$$
\begin{aligned}
& \boldsymbol{V} \times \rho_{0}^{i} \boldsymbol{V}^{i}+\boldsymbol{\varphi} \times\left[\frac{d \rho_{0}^{i}}{d t}\left(\boldsymbol{V}+\boldsymbol{V}^{i}\right)+\rho_{0}^{i} \frac{d\left(\boldsymbol{V}+\boldsymbol{V}^{i}\right)}{d t}\right] \\
= & \boldsymbol{\varphi} \times\left[\rho_{0}^{i} \mathbf{g}+\Pi^{i}\left(\boldsymbol{V}+\boldsymbol{V}^{i}\right)\right] \\
& +\boldsymbol{\varphi} \times \operatorname{Div}\left[\mathbf{P}^{i}-\left(\boldsymbol{V}+\boldsymbol{V}^{i}\right) \otimes \boldsymbol{M}^{i}\right]+\mathcal{E}: \operatorname{Grad} \boldsymbol{\varphi}\left[\mathbf{P}^{i}-\left(\boldsymbol{V}+\boldsymbol{V}^{i}\right) \otimes \boldsymbol{M}^{i}\right]^{\mathrm{T}} \\
= & \boldsymbol{\varphi} \times\left[\rho_{0}^{i} \mathbf{g}+\Pi^{i}\left(\boldsymbol{V}+\boldsymbol{V}^{i}\right)\right] \\
& +\boldsymbol{\varphi} \times\left[\operatorname{Div} \mathbf{P}^{i}-\operatorname{Grad}\left(\boldsymbol{V}+\boldsymbol{V}^{i}\right) \boldsymbol{M}^{i}-\left(\boldsymbol{V}+\boldsymbol{V}^{i}\right) \operatorname{Div} \boldsymbol{M}^{i}\right] \\
& +\mathcal{E}: \operatorname{Grad} \boldsymbol{\varphi}\left[\mathbf{P}^{i}-\left(\boldsymbol{V}+\boldsymbol{V}^{i}\right) \otimes \boldsymbol{M}^{i}\right]^{\mathrm{T}} .
\end{aligned}
$$

This equation can be simplified using (3.2.19):

$$
\begin{aligned}
& \boldsymbol{V} \times \rho_{0}^{i} \boldsymbol{V}^{i}+\boldsymbol{\varphi} \times\left[\frac{d \rho_{0}^{i}}{d t}\left(\boldsymbol{V}+\boldsymbol{V}^{i}\right)\right] \\
= & \boldsymbol{\varphi} \times\left[\Pi^{i}\left(\boldsymbol{V}+\boldsymbol{V}^{i}\right)\right]+\boldsymbol{\varphi} \times\left[-\left(\boldsymbol{V}+\boldsymbol{V}^{i}\right) \operatorname{Div} \boldsymbol{M}^{i}\right]+\mathcal{E}: \operatorname{Grad} \boldsymbol{\varphi}\left[\mathbf{P}^{i}-\left(\boldsymbol{V}+\boldsymbol{V}^{i}\right) \otimes \boldsymbol{M}^{i}\right]^{\mathrm{T}} .
\end{aligned}
$$

Taking (3.2.5) into account, the following relation can be obtained

$$
\boldsymbol{\varphi} \times\left[\frac{d \rho_{0}^{i}}{d t}\left(\boldsymbol{V}+\boldsymbol{V}^{i}\right)\right]=\boldsymbol{\varphi} \times\left[\Pi^{i}\left(\boldsymbol{V}+\boldsymbol{V}^{i}\right)\right]-\boldsymbol{\varphi} \times\left[\left(\boldsymbol{V}+\boldsymbol{V}^{i}\right) \operatorname{Div} \boldsymbol{M}^{i}\right]
$$


The (3.2.28) can be further simplified by substituting the above relation:

$$
\begin{aligned}
\boldsymbol{V} \times \rho_{0}^{i} \boldsymbol{V}^{i} & =\mathcal{E}: \operatorname{Grad} \boldsymbol{\varphi}\left[\mathbf{P}^{i}-\left(\boldsymbol{V}+\boldsymbol{V}^{i}\right) \otimes \boldsymbol{M}^{i}\right]^{\mathrm{T}} \\
& =\mathcal{E}: \mathbf{F}\left[\mathbf{P}^{i}-\left(\boldsymbol{V}+\boldsymbol{V}^{i}\right) \otimes \boldsymbol{M}^{i}\right]^{\mathrm{T}} \stackrel{(\mathrm{A} .1 .11)}{=}-\mathcal{E}:\left\{\mathbf{F}\left[\mathbf{P}^{i}-\left(\boldsymbol{V}+\boldsymbol{V}^{i}\right) \otimes \boldsymbol{M}^{i}\right]^{\mathrm{T}}\right\}^{\mathrm{T}} \\
& =-\mathcal{E}:\left[\mathbf{P}^{i}-\left(\boldsymbol{V}+\boldsymbol{V}^{i}\right) \otimes \boldsymbol{M}^{i}\right] \mathbf{F}^{\mathrm{T}}=-\mathcal{E}:\left[\mathbf{P}^{i} \mathbf{F}^{\mathrm{T}}-\left(\boldsymbol{V}+\boldsymbol{V}^{i}\right) \otimes \boldsymbol{M}^{i} \mathbf{F}^{\mathrm{T}}\right] \\
& =-\mathcal{E}:\left[\mathbf{P}^{i} \mathbf{F}^{\mathrm{T}}-\left(\boldsymbol{V}+\boldsymbol{V}^{i}\right) \otimes \mathbf{F} \boldsymbol{M}^{i}\right]=-\mathcal{E}:\left(\mathbf{P}^{i} \mathbf{F}^{\mathrm{T}}\right)+\mathcal{E}:\left[\left(\boldsymbol{V}+\boldsymbol{V}^{i}\right) \otimes \mathbf{F} \boldsymbol{M}^{i}\right] \\
& =-\mathcal{E}:\left(\mathbf{P}^{i} \mathbf{F}^{\mathrm{T}}\right)+\mathcal{E}:\left(\boldsymbol{V} \otimes \mathbf{F} \boldsymbol{M}^{i}\right)+\mathcal{E}:\left(\boldsymbol{V}^{i} \otimes \mathbf{F} \boldsymbol{M}^{i}\right) \\
& \stackrel{(\mathrm{A} .1 .40)}{=} \mathcal{E}:\left(-\mathbf{P}^{i} \mathbf{F}^{\mathrm{T}}+\boldsymbol{V}^{i} \otimes \mathbf{F} \boldsymbol{M}^{i}\right)+\boldsymbol{V} \times\left(\mathbf{F} \boldsymbol{M}^{i}\right)
\end{aligned}
$$

where $\mathbf{F}=\operatorname{Grad} \varphi$.

The left-hand side of $(3.2 .29)$ is

$$
\text { LHS }=\boldsymbol{V} \times \rho_{0}^{i} \boldsymbol{V}^{i} \stackrel{(3.2 .11)}{=} \boldsymbol{V} \times \rho_{0}^{i} \frac{\mathbf{F} \boldsymbol{M}^{i}}{\rho_{0}^{i}}=\boldsymbol{V} \times \mathbf{F} \boldsymbol{M}^{i} .
$$

Thus, we can conclude

$$
\boldsymbol{O}=\mathcal{E}:\left(-\mathbf{P}^{i} \mathbf{F}^{\mathrm{T}}+\boldsymbol{V}^{i} \otimes \mathbf{F} \boldsymbol{M}^{i}\right)
$$

Substituting $\boldsymbol{M}^{i}=\rho_{0}^{i} \mathbf{F}^{-1} \boldsymbol{V}^{i}$ from (C.1.2) into it:

$$
\begin{aligned}
\boldsymbol{O} & =\mathcal{E}:\left(-\mathbf{P}^{i} \mathbf{F}^{\mathrm{T}}+\rho_{0}^{i} \boldsymbol{V}^{i} \otimes \mathbf{F F}^{-1} \boldsymbol{V}^{i}\right) \\
& =\mathcal{E}:\left(-\mathbf{P}^{i} \mathbf{F}^{\mathrm{T}}+\rho_{0}^{i} \boldsymbol{V}^{i} \otimes \boldsymbol{V}^{i}\right)
\end{aligned}
$$

From the above result the symmetry of the expression $\mathbf{P}^{i} \mathbf{F}^{\mathrm{T}}-\rho_{0}^{i} \boldsymbol{V}^{i} \otimes \boldsymbol{V}^{i}$ can be concluded. Therefore, the first term $\mathbf{P}^{i} \mathbf{F}^{\mathrm{T}}$ should be symmetry:

$$
\mathbf{P}^{i} \mathbf{F}^{\mathrm{T}}=\mathbf{F}\left(\mathbf{P}^{i}\right)^{\mathrm{T}}
$$

and with it

$$
\boldsymbol{\sigma}^{i}=J^{-1} \mathbf{P}^{i} \mathbf{F}^{\mathrm{T}}=J^{-1} \mathbf{F}\left(\mathbf{P}^{i}\right)^{\mathrm{T}}=\left(\boldsymbol{\sigma}^{i}\right)^{\mathrm{T}}
$$

the symmetry of the partial Cauchy stress tensor of species $i$ is deduced in spite of mass flux. Accordingly the partial second Piola-Kirchhoff stress tensor of species $i \mathbf{S}^{i}$ can be shown:

$$
\left(\mathbf{S}^{i}\right)^{\mathrm{T}}=J \mathbf{F}^{-1}\left(\boldsymbol{\sigma}^{i}\right)^{\mathrm{T}} \mathbf{F}^{-\mathrm{T}}=J \mathbf{F}^{-1} \boldsymbol{\sigma}^{i} \mathbf{F}^{-\mathrm{T}}=\mathbf{S}^{i} .
$$


The balance of angular momentum of the whole system is obtained by summing up over all species of $(3.2 .27)$

$$
\begin{aligned}
\sum_{i} \frac{d}{d t} \int_{\Omega_{0}} \boldsymbol{\varphi} \times \rho_{0}^{i}\left(\boldsymbol{V}+\boldsymbol{V}^{i}\right) d V & =\sum_{i} \int_{\Omega_{0}} \boldsymbol{\varphi} \times\left[\rho_{0}^{i} \mathbf{g}+\Pi^{i}\left(\boldsymbol{V}+\boldsymbol{V}^{i}\right)\right] d V \\
& +\sum_{i} \int_{\partial \Omega_{0}} \boldsymbol{\varphi} \times\left[\mathbf{P}^{i}-\left(\boldsymbol{V}+\boldsymbol{V}^{i}\right) \otimes \boldsymbol{M}^{i}\right] \cdot \boldsymbol{N} d A .
\end{aligned}
$$

The left-hand side of $(3.2 .27)$ is

$$
\begin{aligned}
\text { LHS } & =\sum_{i} \int_{\Omega_{0}}\left\{\frac{d \boldsymbol{\varphi}}{d t} \times \rho_{0}^{i}\left(\boldsymbol{V}+\boldsymbol{V}^{i}\right)+\boldsymbol{\varphi} \times \frac{d}{d t}\left[\rho_{0}^{i}\left(\boldsymbol{V}+\boldsymbol{V}^{i}\right)\right]\right\} d V \\
& =\int_{\Omega_{0}}\left\{\frac{d \boldsymbol{\varphi}}{d t} \times\left[\sum_{i} \rho_{0}^{i} \boldsymbol{V}+\sum_{i}\left(\rho_{0}^{i} \boldsymbol{V}^{i}\right)\right]+\boldsymbol{\varphi} \times \frac{d}{d t} \sum_{i}\left[\rho_{0}^{i}\left(\boldsymbol{V}+\boldsymbol{V}^{i}\right)\right]\right\} d V \\
& =\int_{\Omega_{0}}\left\{\frac{d \boldsymbol{\varphi}}{d t} \times\left[\rho_{0} \boldsymbol{V}+\sum_{i}\left(\rho_{0}^{i} \boldsymbol{V}^{i}\right)\right]+\boldsymbol{\varphi} \times \frac{d}{d t}\left[\rho_{0} \boldsymbol{V}+\sum_{i}\left(\rho_{0}^{i} \boldsymbol{V}^{i}\right)\right]\right\} d V .
\end{aligned}
$$

The right-hand side of $(3.2 .27)$ is

RHS

$=\int_{\Omega_{0}} \boldsymbol{\varphi} \times \sum_{i}\left[\rho_{0}^{i} \mathbf{g}+\Pi^{i}\left(\boldsymbol{V}+\boldsymbol{V}^{i}\right)\right] d V+\int_{\partial \Omega_{0}} \boldsymbol{\varphi} \times \sum_{i}\left[\mathbf{P}^{i}-\left(\boldsymbol{V}+\boldsymbol{V}^{i}\right) \otimes \boldsymbol{M}^{i}\right] \cdot \boldsymbol{N} d A$
$=\int_{\Omega_{0}} \boldsymbol{\varphi} \times\left[\sum_{i} \rho_{0}^{i} \mathbf{g}+\sum_{i} \Pi^{i}\left(\boldsymbol{V}+\boldsymbol{V}^{i}\right)\right] d V+\int_{\partial \Omega_{0}} \boldsymbol{\varphi} \times\left[\sum_{i} \mathbf{P}^{i}-\sum_{i}\left(\boldsymbol{V}+\boldsymbol{V}^{i}\right) \otimes \boldsymbol{M}^{i}\right] \cdot \boldsymbol{N} d A$

$\stackrel{(3.2 .2)}{=} \int_{\Omega_{0}} \boldsymbol{\varphi} \times\left[\rho_{0} \mathbf{g}+\sum_{i} \Pi^{i}\left(\boldsymbol{V}+\boldsymbol{V}^{i}\right)\right] d V+\int_{\partial \Omega_{0}} \boldsymbol{\varphi} \times\left[\mathbf{P}-\sum_{i}\left(\boldsymbol{V}+\boldsymbol{V}^{i}\right) \otimes \boldsymbol{M}^{i}\right] \cdot \boldsymbol{N} d A$

$\stackrel{(\text { A.4.1) }}{=} \int_{\Omega_{0}} \boldsymbol{\varphi} \times\left[\rho_{0} \mathbf{g}+\sum_{i} \Pi^{i}\left(\boldsymbol{V}+\boldsymbol{V}^{i}\right)\right] d V$

$+\int_{\Omega_{0}}\left\{\boldsymbol{\varphi} \times \operatorname{Div}\left[\mathbf{P}-\sum_{i}\left(\boldsymbol{V}+\boldsymbol{V}^{i}\right) \otimes \boldsymbol{M}^{i}\right]+\mathcal{E}: \operatorname{Grad} \boldsymbol{\varphi}\left[\mathbf{P}-\sum_{i}\left(\boldsymbol{V}+\boldsymbol{V}^{i}\right) \otimes \boldsymbol{M}^{i}\right]^{\mathrm{T}}\right\} d V$.

By equating the both sides and considering (3.2.23) the following equation can be obtained:

$$
\frac{d \boldsymbol{\varphi}}{d t} \times\left[\rho_{0} \boldsymbol{V}+\sum_{i}\left(\rho_{0}^{i} \boldsymbol{V}^{i}\right)\right]=\mathcal{E}: \operatorname{Grad} \boldsymbol{\varphi}\left[\mathbf{P}-\sum_{i}\left(\boldsymbol{V}+\boldsymbol{V}^{i}\right) \otimes \boldsymbol{M}^{i}\right]^{\mathrm{T}}
$$

The left-hand side of $(3.2 .35)$ is

$$
\mathrm{LHS}=\boldsymbol{V} \times \sum_{i}\left(\rho_{0}^{i} \boldsymbol{V}^{i}\right) \stackrel{(3.2 .11)}{=} \boldsymbol{V} \times \sum_{i}\left(\mathbf{F} \boldsymbol{M}^{i}\right)
$$


and its right-hand side is

$$
\begin{aligned}
\mathrm{RHS} & =\mathcal{E}: \mathbf{F}\left[\mathbf{P}-\sum_{i}\left(\boldsymbol{V}+\boldsymbol{V}^{i}\right) \otimes \boldsymbol{M}^{i}\right]^{\mathrm{T}} \\
& =-\mathcal{E}:\left\{\mathbf{F}\left[\mathbf{P}-\sum_{i}\left(\boldsymbol{V}+\boldsymbol{V}^{i}\right) \otimes \boldsymbol{M}^{i}\right]^{\mathrm{T}}\right\}^{\mathrm{T}} \\
& =-\mathcal{E}:\left[\mathbf{P}-\sum_{i}\left(\boldsymbol{V}+\boldsymbol{V}^{i}\right) \otimes \boldsymbol{M}^{i}\right] \mathbf{F}^{\mathrm{T}} \\
& =-\mathcal{E}:\left[\mathbf{P} \mathbf{F}^{\mathrm{T}}-\sum_{i}\left(\boldsymbol{V}+\boldsymbol{V}^{i}\right) \otimes \boldsymbol{M}^{i} \mathbf{F}^{\mathrm{T}}\right] \\
& =-\mathcal{E}:\left[\mathbf{P} \mathbf{F}^{\mathrm{T}}-\sum_{i}\left(\boldsymbol{V}+\boldsymbol{V}^{i}\right) \otimes \mathbf{F} \boldsymbol{M}^{i}\right] \\
& =-\mathcal{E}: \mathbf{P F}+\mathcal{E}: \sum_{i}\left(\boldsymbol{V}+\boldsymbol{V}^{i}\right) \otimes \mathbf{F} \boldsymbol{M}^{i} \\
& =-\mathcal{E}: \mathbf{P} \mathbf{F}^{\mathrm{T}}+\mathcal{E}: \sum_{i}\left(\boldsymbol{V} \otimes \mathbf{F} \boldsymbol{M}^{i}+\boldsymbol{V}^{i} \otimes \mathbf{F} \boldsymbol{M}^{i}\right) \\
& =\mathcal{E}:\left(-\mathbf{P F}^{\mathrm{T}}+\sum_{i} \boldsymbol{V}^{i} \otimes \mathbf{F} \boldsymbol{M}^{i}\right)+\mathcal{E}: \sum_{i}\left(\boldsymbol{V} \otimes \mathbf{F} \boldsymbol{M}^{i}\right) \\
& =\mathcal{E}:\left(-\mathbf{P} \mathbf{F}^{\mathrm{T}}+\sum_{i} \boldsymbol{V}^{i} \otimes \mathbf{F} \boldsymbol{M}^{i}\right)+\mathcal{E}:\left(\boldsymbol{V} \otimes \sum_{i} \mathbf{F} \boldsymbol{M}^{i}\right) \\
& =\mathcal{E}:\left(\mathbf{P F}^{\mathrm{T}}+\sum_{i} \boldsymbol{V}^{i} \otimes \mathbf{F} \boldsymbol{M}^{i}\right)+\boldsymbol{V} \times \sum_{i} \mathbf{F} \boldsymbol{M}^{i} .
\end{aligned}
$$

By equating the both sides

$$
\mathbf{0}=\mathcal{E}:\left(-\mathbf{P F}^{\mathrm{T}}+\sum_{i} \boldsymbol{V}^{i} \otimes \mathbf{F} \boldsymbol{M}^{i}\right),
$$

and substituting $\boldsymbol{M}^{i}=\rho_{0}^{i} \mathbf{F}^{-1} \boldsymbol{V}^{i}$

$$
\begin{aligned}
\mathbf{0} & =\mathcal{E}:\left(-\mathbf{P F}^{\mathrm{T}}+\sum_{i} \boldsymbol{V}^{i} \otimes \mathbf{F} \rho_{0}^{i} \mathbf{F}^{-1} \boldsymbol{V}^{i}\right) \\
& =\mathcal{E}:\left(-\mathbf{P F}^{\mathrm{T}}+\rho_{0}^{i} \sum_{i} \boldsymbol{V}^{i} \otimes \boldsymbol{V}^{i}\right),
\end{aligned}
$$

we can conclude the symmetry of the expression $\mathbf{P F}^{\mathrm{T}}-\rho_{0}^{i} \boldsymbol{V}^{i} \otimes \boldsymbol{V}^{i}$.

Therefore, the first term $\mathbf{P F}^{\mathrm{T}}$ should be symmetry:

$$
\mathbf{P F}^{\mathrm{T}}=\mathbf{F P}^{\mathrm{T}}
$$


and with it

$$
\boldsymbol{\sigma}=J^{-1} \mathbf{P} \mathbf{F}^{\mathrm{T}}=J^{-1} \mathbf{F}(\mathbf{P})^{\mathrm{T}}=(\boldsymbol{\sigma})^{\mathrm{T}}
$$

the symmetry of the Cauchy stress tensor of the system is deduced in spite of mass fluxes. Accordingly the symmetry of the second Piola-Kirchhoff stress tensor of the system $\mathbf{S}$ can be shown:

$$
\mathbf{S}^{\mathrm{T}}=J \mathbf{F}^{-1} \boldsymbol{\sigma}^{\mathrm{T}} \mathbf{F}^{-\mathrm{T}}=J \mathbf{F}^{-1} \boldsymbol{\sigma} \mathbf{F}^{-\mathrm{T}}=\mathbf{S}
$$

\subsubsection{Balance of energy}

The balance of energy states that the rate of energy $\dot{\mathcal{E}}(\mathcal{E}$ is the total energy of the body and a sum of the kinetic energy $\mathcal{K}$ and the internal energy $\mathcal{I}$ ) is balanced with the power of external forces $\mathcal{P}$ and the heat supply rate $\mathcal{Q}$ :

$$
\dot{\mathcal{E}}=\frac{d}{d t}(\mathcal{K}+\mathcal{I})=\mathcal{P}+\mathcal{Q}
$$

The power of external forces and the heat supply rate are divided into closed and open part denoted by subscript closed and open, respectively. The closed part are the familiar terms known from classical continuum mechanics. Due to the mass source and flux the open part should be added to the closed part . An additional energy is assumed to be transferred from the other species to the species $i \tilde{E}^{i}$. Then the energy balance equation is

$$
\frac{d}{d t}(\mathcal{K}+\mathcal{I})=\mathcal{P}_{\text {closed }}+\mathcal{Q}_{\text {closed }}+\mathcal{P}_{\text {open }}+\mathcal{Q}_{\text {open }}+\mathcal{Q}_{\text {transfer }}
$$

The kinetic and internal energy of species $i$ are

$$
\begin{aligned}
& \mathcal{K}=\int_{\Omega_{0}} \rho_{0}^{i}\left[\frac{1}{2}\left(\boldsymbol{V}+\boldsymbol{V}^{i}\right)^{2}\right] d V, \\
& \mathcal{I}=\int_{\Omega_{0}} \rho_{0}^{i} E^{i} d V .
\end{aligned}
$$


If the heat supply to species $i$ per unit mass is $R^{i}$ and the partial heat flux of species $i$ through the surface is $H^{i}\left(=-\mathbf{Q}^{i} \cdot \mathbf{N}, \mathbf{Q}^{i}\right.$ the partial heat flux vector $)$, the power of external forces and the heat supply rate of the closed part are

$$
\begin{aligned}
& \mathcal{P}_{\text {closed }}=\int_{\Omega_{0}} \rho_{0}^{i} \mathbf{g} \cdot\left(\boldsymbol{V}+\boldsymbol{V}^{i}\right) d V+\int_{\partial \Omega_{0}} \boldsymbol{T}^{i} \cdot\left(\boldsymbol{V}+\boldsymbol{V}^{i}\right) d A, \\
& \mathcal{Q}_{\text {closed }}=\int_{\Omega_{0}} \rho_{0}^{i} R^{i} d V+\int_{\partial \Omega_{0}} H^{i} d A .
\end{aligned}
$$

The power of external forces and the heat supply rate of the open part and the energy transfer from other species to species $i$ are

$$
\begin{aligned}
\mathcal{P}_{\text {open }} & =\int_{\Omega_{0}} \frac{1}{2}\left(\boldsymbol{V}+\boldsymbol{V}^{i}\right)^{2} \Pi^{i} d V-\int_{\partial \Omega_{0}} \frac{1}{2}\left(\boldsymbol{V}+\boldsymbol{V}^{i}\right)^{2} \boldsymbol{M}^{i} \cdot \boldsymbol{N} d A, \\
\mathcal{Q}_{\text {open }} & =\int_{\Omega_{0}} \Pi^{i} E^{i} d V-\int_{\partial \Omega_{0}} E^{i} \boldsymbol{M}^{i} \cdot \boldsymbol{N} d A, \\
\mathcal{Q}_{\text {transfer }} & =\int_{\Omega_{0}} \rho_{0}^{i} \tilde{E}^{i} d V .
\end{aligned}
$$

The balance of energy is

$$
\begin{aligned}
& \frac{d}{d t} \int_{\Omega_{0}} \rho_{0}^{i}\left[E^{i}+\frac{1}{2}\left(\boldsymbol{V}+\boldsymbol{V}^{i}\right)^{2}\right] d V \\
= & \int_{\Omega_{0}} \rho_{0}^{i} \mathbf{g} \cdot\left(\boldsymbol{V}+\boldsymbol{V}^{i}\right) d V+\int_{\partial \Omega_{0}} \boldsymbol{T}^{i} \cdot\left(\boldsymbol{V}+\boldsymbol{V}^{i}\right) d A+\int_{\Omega_{0}} \rho_{0}^{i} R^{i} d V+\int_{\partial \Omega_{0}} H^{i} d A \\
& +\int_{\Omega_{0}} \Pi^{i}\left[E^{i}+\frac{1}{2}\left(\boldsymbol{V}+\boldsymbol{V}^{i}\right)^{2}\right] d V-\int_{\partial \Omega_{0}} \boldsymbol{M}^{i}\left[E^{i}+\frac{1}{2}\left(\boldsymbol{V}+\boldsymbol{V}^{i}\right)^{2}\right] \cdot \boldsymbol{N} d A \\
& +\int_{\Omega_{0}} \rho_{0}^{i} \tilde{E}^{i} d V .
\end{aligned}
$$

The left-hand side of Eq. (3.2.42) is

$$
\begin{aligned}
\text { LHS } & =\int_{\Omega_{0}}\left\{\frac{d \rho_{0}^{i}}{d t}\left[E^{i}+\frac{1}{2}\left(\boldsymbol{V}+\boldsymbol{V}^{i}\right)^{2}\right]+\rho_{0}^{i} \frac{d}{d t}\left[E^{i}+\frac{1}{2}\left(\boldsymbol{V}+\boldsymbol{V}^{i}\right)^{2}\right]\right\} d V \\
& =\int_{\Omega_{0}}\left\{\frac{d \rho_{0}^{i}}{d t}\left[E^{i}+\frac{1}{2}\left(\boldsymbol{V}+\boldsymbol{V}^{i}\right)^{2}\right]+\rho_{0}^{i} \frac{d E^{i}}{d t}+\rho_{0}^{i}\left(\boldsymbol{V}+\boldsymbol{V}^{i}\right) \frac{d\left(\boldsymbol{V}+\boldsymbol{V}^{i}\right)}{d t}\right\} d V,
\end{aligned}
$$

and the right-hand side of Eq. (3.2.42) is

$$
\begin{aligned}
\mathrm{RHS}= & \int_{\Omega_{0}} \rho_{0}^{i} \mathbf{g} \cdot\left(\boldsymbol{V}+\boldsymbol{V}^{i}\right) d V+\int_{\partial \Omega_{0}}\left(\boldsymbol{V}+\boldsymbol{V}^{i}\right) \cdot \mathbf{P}^{i} \boldsymbol{N} d A+\int_{\Omega_{0}} \rho_{0}^{i} R^{i} d V-\int_{\partial \Omega_{0}} \mathbf{Q}^{i} \cdot \boldsymbol{N} d A \\
& +\int_{\Omega_{0}} \Pi^{i}\left[E^{i}+\frac{1}{2}\left(\boldsymbol{V}+\boldsymbol{V}^{i}\right)^{2}\right] d V-\int_{\partial \Omega_{0}} \boldsymbol{M}^{i}\left[E^{i}+\frac{1}{2}\left(\boldsymbol{V}+\boldsymbol{V}^{i}\right)^{2}\right] \cdot \boldsymbol{N} d A+\int_{\Omega_{0}} \rho_{0}^{i} \tilde{E}^{i} d V .
\end{aligned}
$$


The second term of the right-hand side is

$$
\begin{aligned}
& \int_{\partial \Omega_{0}}\left(\boldsymbol{V}+\boldsymbol{V}^{i}\right) \cdot \mathbf{P}^{i} \boldsymbol{N} d A=\int_{\Omega_{0}} \operatorname{Div}\left[\left(\boldsymbol{V}+\boldsymbol{V}^{i}\right) \mathbf{P}^{i}\right] d V \\
= & \int_{\Omega_{0}}\left[\operatorname{Grad}\left(\boldsymbol{V}+\boldsymbol{V}^{i}\right): \mathbf{P}^{i}+\left(\boldsymbol{V}+\boldsymbol{V}^{i}\right) \cdot \operatorname{Div} \mathbf{P}^{i}\right] d V,
\end{aligned}
$$

and sixth term of the right-hand side is

$$
\begin{aligned}
& \int_{\partial \Omega_{0}} \boldsymbol{M}^{i}\left[E^{i}+\frac{1}{2}\left(\boldsymbol{V}+\boldsymbol{V}^{i}\right)^{2}\right] \cdot \boldsymbol{N} d A=\int_{\Omega_{0}} \operatorname{Div}\left\{\boldsymbol{M}^{i}\left[E^{i}+\frac{1}{2}\left(\boldsymbol{V}+\boldsymbol{V}^{i}\right)^{2}\right]\right\} d V \\
= & \int_{\Omega_{0}}\left\{\left[E^{i}+\frac{1}{2}\left(\boldsymbol{V}+\boldsymbol{V}^{i}\right)^{2}\right] \operatorname{Div} \boldsymbol{M}^{i}+\operatorname{Grad}\left[E^{i}+\frac{1}{2}\left(\boldsymbol{V}+\boldsymbol{V}^{i}\right)^{2}\right] \cdot \boldsymbol{M}^{i}\right\} d V .
\end{aligned}
$$

Then the balance equation can be written as

$$
\begin{aligned}
& \int_{\Omega_{0}}\left\{\frac{d \rho_{0}^{i}}{d t}\left[E^{i}+\frac{1}{2}\left(\boldsymbol{V}+\boldsymbol{V}^{i}\right)^{2}\right]+\rho_{0}^{i} \frac{d E^{i}}{d t}+\rho_{0}^{i}\left(\boldsymbol{V}+\boldsymbol{V}^{i}\right) \frac{d\left(\boldsymbol{V}+\boldsymbol{V}^{i}\right)}{d t}\right\} d V \\
= & \int_{\Omega_{0}} \rho_{0}^{i} \mathbf{g} \cdot\left(\boldsymbol{V}+\boldsymbol{V}^{i}\right) d V+\int_{\Omega_{0}}\left[\operatorname{Grad}\left(\boldsymbol{V}+\boldsymbol{V}^{i}\right): \mathbf{P}^{i}+\left(\boldsymbol{V}+\boldsymbol{V}^{i}\right) \cdot \operatorname{Div} \mathbf{P}^{i}\right] d V \\
& +\int_{\Omega_{0}} \rho_{0}^{i} R^{i} d V-\int_{\partial \Omega_{0}} \mathbf{Q}^{i} \cdot \boldsymbol{N} d A+\int_{\Omega_{0}} \Pi^{i}\left[E^{i}+\frac{1}{2}\left(\boldsymbol{V}+\boldsymbol{V}^{i}\right)^{2}\right] d V \\
& -\int_{\Omega_{0}}\left\{\left[E^{i}+\frac{1}{2}\left(\boldsymbol{V}+\boldsymbol{V}^{i}\right)^{2}\right] \operatorname{Div} \boldsymbol{M}^{i}+\operatorname{Grad}\left[E^{i}+\frac{1}{2}\left(\boldsymbol{V}+\boldsymbol{V}^{i}\right)^{2}\right] \cdot \boldsymbol{M}^{i}\right\} d V \\
& +\int_{\Omega_{0}} \rho_{0}^{i} \tilde{E}^{i} d V .
\end{aligned}
$$

This equation can be simplified by applying (3.2.5). Multiplying (3.2.5) by $\left[E^{i}+\frac{1}{2}\left(\boldsymbol{V}+\boldsymbol{V}^{i}\right)^{2}\right]$ gives

$$
\frac{d \rho_{0}^{i}}{d t}\left[E^{i}+\frac{1}{2}\left(\boldsymbol{V}+\boldsymbol{V}^{i}\right)^{2}\right]=\left(\Pi^{i}-\operatorname{Div} \boldsymbol{M}^{i}\right)\left[E^{i}+\frac{1}{2},\left(\boldsymbol{V}+\boldsymbol{V}^{i}\right)^{2}\right]
$$

and substracting (3.2.44) from (3.2.43) gives 


$$
\begin{aligned}
& \int_{\Omega_{0}}\left\{\rho_{0}^{i} \frac{d E^{i}}{d t}+\rho_{0}^{i}\left(\boldsymbol{V}+\boldsymbol{V}^{i}\right) \frac{d\left(\boldsymbol{V}+\boldsymbol{V}^{i}\right)}{d t}\right\} d V \\
= & \int_{\Omega_{0}} \rho_{0}^{i} \mathbf{g} \cdot\left(\boldsymbol{V}+\boldsymbol{V}^{i}\right) d V+\int_{\Omega_{0}}\left[\operatorname{Grad}\left(\boldsymbol{V}+\boldsymbol{V}^{i}\right): \mathbf{P}^{i}+\left(\boldsymbol{V}+\boldsymbol{V}^{i}\right) \cdot \operatorname{Div} \mathbf{P}^{i}\right] d V \\
& +\int_{\Omega_{0}} \rho_{0}^{i} R^{i} d V-\int_{\partial \Omega_{0}} \mathbf{Q}^{i} \cdot \boldsymbol{N} d A-\int_{\Omega_{0}}\left\{\operatorname{Grad}\left[E^{i}+\frac{1}{2}\left(\boldsymbol{V}+\boldsymbol{V}^{i}\right)^{2}\right] \cdot \boldsymbol{M}^{i}\right\} d V \\
& +\int_{\Omega_{0}} \rho_{0}^{i} \tilde{E}^{i} d V .
\end{aligned}
$$

Using the balance of linear momentum Eq. (3.2.19) multiplied by $\left(\boldsymbol{V}+\boldsymbol{V}^{i}\right)$ and the following identity:

$$
\begin{aligned}
& \operatorname{Grad}\left[\frac{1}{2}\left(\boldsymbol{V}+\boldsymbol{V}^{i}\right)^{2}\right] \cdot \boldsymbol{M}^{i}=\frac{1}{2} \operatorname{Grad}\left(\boldsymbol{V}+\boldsymbol{V}^{i}\right)^{2} \cdot \boldsymbol{M}^{i} \\
= & {\left[\operatorname{Grad}^{\mathrm{T}}\left(\boldsymbol{V}+\boldsymbol{V}^{i}\right)\left(\boldsymbol{V}+\boldsymbol{V}^{i}\right)\right] \cdot \boldsymbol{M}^{i}=\left(\boldsymbol{V}+\boldsymbol{V}^{i}\right) \operatorname{Grad}\left(\boldsymbol{V}+\boldsymbol{V}^{i}\right) \cdot \boldsymbol{M}^{i}, }
\end{aligned}
$$

the energy balance equation can be obtained

$$
\begin{aligned}
\int_{\Omega_{0}} \rho_{0}^{i} \frac{d E^{i}}{d t} d V= & \int_{\Omega_{0}}\left[\operatorname{Grad}\left(\boldsymbol{V}+\boldsymbol{V}^{i}\right): \mathbf{P}^{i}\right] d V+\int_{\Omega_{0}} \rho_{0}^{i} R^{i} d V-\int_{\partial \Omega_{0}} \mathbf{Q}^{i} \cdot \boldsymbol{N} d A \\
& -\int_{\Omega_{0}}\left(\operatorname{Grad} E^{i} \cdot \boldsymbol{M}^{i}\right) d V+\int_{\Omega_{0}} \rho_{0}^{i} \tilde{E}^{i} d V .
\end{aligned}
$$

Using the divergence theorem, a differential form is obtained:

$$
\rightarrow \quad \rho_{0}^{i} \frac{d E^{i}}{d t}=\operatorname{Grad}\left(\boldsymbol{V}+\boldsymbol{V}^{i}\right): \mathbf{P}^{i}+\rho_{0}^{i} R^{i}-\operatorname{Div} \mathbf{Q}^{i}-\operatorname{Grad} E^{i} \cdot \boldsymbol{M}^{i}+\rho_{0}^{i} \tilde{E}^{i}
$$

Summing up over $i$ gives the balance of energy for the whole system:

$$
\sum_{i} \rho_{0}^{i} \frac{d E^{i}}{d t}=\sum_{i}\left[\operatorname{Grad}\left(\boldsymbol{V}+\boldsymbol{V}^{i}\right): \mathbf{P}^{i}+\rho_{0}^{i} R^{i}-\operatorname{Div} \mathbf{Q}^{i}-\operatorname{Grad} E^{i} \cdot \boldsymbol{M}^{i}+\rho_{0}^{i} \tilde{E}^{i}\right] .
$$


If we assume that the energy of the system relies on only the interaction with its environment, then the mass inter-conversion $\Pi^{i}$ and energy transfer $\tilde{E}^{i}$ between species have no effect on the energy rate of the system:

$$
\begin{aligned}
& \sum_{i} \int_{\Omega_{0}} \Pi^{i}\left[E^{i}+\frac{1}{2}\left(\boldsymbol{V}+\boldsymbol{V}^{i}\right)^{2}\right] d V+\sum_{i} \int_{\Omega_{0}} \rho_{0}^{i} \tilde{E}^{i} d V=0 \\
\rightarrow & \sum_{i}\left\{\Pi^{i}\left[E^{i}+\frac{1}{2}\left(\boldsymbol{V}+\boldsymbol{V}^{i}\right)^{2}\right]+\rho_{0}^{i} \tilde{E}^{i}\right\}=0 \\
\rightarrow & \sum_{i}\left(\Pi^{i} E^{i}+\frac{1}{2} \Pi^{i} \boldsymbol{V}^{2}+\Pi^{i} \boldsymbol{V} \cdot \boldsymbol{V}^{i}+\frac{1}{2} \Pi^{i}\left(\boldsymbol{V}^{i}\right)^{2}+\rho_{0}^{i} \tilde{E}^{i}\right)=0 .
\end{aligned}
$$

From the two assumptions (3.2.6) and (3.2.22), we obtain

$$
\sum_{i}\left(\Pi^{i} E^{i}+\frac{1}{2} \Pi^{i}\left(\boldsymbol{V}^{i}\right)^{2}+\rho_{0}^{i} \tilde{E}^{i}\right)=0
$$

Considering this relation, the following equation can be deduced from Eq. (3.2.46)

$$
\sum_{i} \rho_{0}^{i} \frac{d E^{i}}{d t}=\sum_{i}\left[\left(\dot{\mathbf{F}}+\operatorname{Grad} \boldsymbol{V}^{i}\right): \mathbf{P}^{i}+\rho_{0}^{i} R^{i}-\operatorname{Div} \mathbf{Q}^{i}-\operatorname{Grad} E^{i} \cdot \boldsymbol{M}^{i}-\Pi^{i}\left(E^{i}+\frac{1}{2}\left(\boldsymbol{V}^{i}\right)^{2}\right)\right]
$$

where the following identity is applied

$$
\operatorname{Grad} \boldsymbol{V}=\frac{\partial \boldsymbol{V}}{\partial \boldsymbol{X}}=\frac{\partial}{\partial \boldsymbol{X}}\left(\frac{\partial \boldsymbol{\varphi}}{\partial t}\right)=\frac{\partial}{\partial t}\left(\frac{\partial \boldsymbol{\varphi}}{\partial \boldsymbol{X}}\right)=\dot{\mathbf{F}}
$$

\subsubsection{Entropy inequality}

The entropy inequality states that the production of entropy is non-negative. The time rate of change of the total entropy $\mathcal{N}$ is equal to or greater than the sum of the entropy exchange due to contact entropy flux and the at-a-distance entropy exchange that can be divided into closed and open part denoted by subscript closed and open, respectively. The closed part $\mathcal{S}_{\text {closed }}$ is the common terms known from classical continuum mechanics. The mass source and flux cause the open part of the entropy change $\mathcal{S}_{\text {open }}$. An additional entropy exchange is introduced that 
is transferred from the other species to the species $i \mathcal{S}_{\text {transfer }}$.

Then, the entropy inequality is given by

$$
\frac{d \mathcal{N}}{d t} \geq \mathcal{S}_{\text {closed }}+\mathcal{S}_{\text {open }}+\mathcal{S}_{\text {transfer }}
$$

The entropy of the species $i$ is

$$
\mathcal{N}=\int_{\Omega_{0}} \rho_{0}^{i} N^{i} d V
$$

the closed and open part of entropy exchange are, respectively,

$$
\begin{aligned}
\mathcal{S}_{\text {closed }} & =\int_{\Omega_{0}} \frac{\rho_{0}^{i} R^{i}}{\Theta} d V-\int_{\partial \Omega_{0}} \frac{\mathbf{Q}^{i}}{\Theta} \cdot \mathbf{N} d A, \\
\mathcal{S}_{\text {open }} & =\int_{\Omega_{0}} \Pi^{i} N^{i} d V-\int_{\partial \Omega_{0}} \boldsymbol{M}^{i} \cdot \mathbf{N} N^{i} d A,
\end{aligned}
$$

and the entropy transferred to species $i$ by all other species $\tilde{\mathcal{N}}^{i}$

$$
\mathcal{S}_{\text {transfer }}=\int_{\Omega_{0}} \rho_{0}^{i} \tilde{\mathcal{N}}^{i} d V
$$

The entropy inequality becomes :D

$$
\begin{aligned}
\frac{d}{d t} \int_{\Omega_{0}} \rho_{0}^{i} N^{i} d V \geq & \int_{\Omega_{0}} \frac{\rho_{0}^{i} R^{i}}{\Theta} d V-\int_{\partial \Omega_{0}} \frac{\mathbf{Q}^{i}}{\Theta} \cdot \mathbf{N} d A+\int_{\Omega_{0}} \Pi^{i} N^{i} d V-\int_{\partial \Omega_{0}} \boldsymbol{M}^{i} \cdot \mathbf{N} N^{i} d A \\
& +\int_{\Omega_{0}} \rho_{0}^{i} \tilde{\mathcal{N}}^{i} d V \\
= & \int_{\Omega_{0}}\left(\frac{\rho_{0}^{i} R^{i}}{\Theta}+\Pi^{i} N^{i}+\rho_{0}^{i} \tilde{\mathcal{N}}^{i}\right) d V-\int_{\partial \Omega_{0}}\left(\frac{\mathbf{Q}^{i}}{\Theta}+\boldsymbol{M}^{i} N^{i}\right) \cdot \mathbf{N} d A \\
= & \int_{\Omega_{0}}\left[\frac{\rho_{0}^{i} R^{i}}{\Theta}+\Pi^{i} N^{i}+\rho_{0}^{i} \tilde{\mathcal{N}}^{i}-\operatorname{Div}\left(\frac{\mathbf{Q}^{i}}{\Theta}+\boldsymbol{M}^{i} N^{i}\right)\right] d V \\
\rightarrow \quad \frac{d\left(\rho_{0}^{i} N^{i}\right)}{d t} \geq & \frac{\rho_{0}^{i} R^{i}}{\Theta}+\Pi^{i} N^{i}+\rho_{0}^{i} \tilde{\mathcal{N}}^{i}-\operatorname{Div}\left(\frac{\mathbf{Q}^{i}}{\Theta}+\boldsymbol{M}^{i} N^{i}\right)
\end{aligned}
$$

Considering Eq. (3.2.5), the inequality can be simplified as follows

$$
\rightarrow \quad \rho_{0}^{i} \frac{d N^{i}}{d t} \geq \frac{\rho_{0}^{i} R^{i}}{\Theta}+\rho_{0}^{i} \tilde{\mathcal{N}}^{i}-\frac{\operatorname{Div} \mathbf{Q}^{i}}{\Theta}+\frac{\operatorname{Grad} \Theta \cdot \mathbf{Q}^{i}}{\Theta^{2}}-\boldsymbol{M}^{i} \cdot \operatorname{Grad} N^{i},
$$

where for the divergence of a product of a scalar-valued function and a vector-valued function the Eq. (A.4.5) and the following identity are applied

$$
\operatorname{Grad} \frac{1}{\Theta}=\frac{\partial}{\partial X^{i}}\left(\frac{1}{\Theta}\right) \mathbf{g}^{i}=\frac{\partial}{\partial \Theta}\left(\frac{1}{\Theta}\right) \frac{\partial \Theta}{\partial X^{i}} \mathbf{g}^{i}=-\frac{1}{\Theta^{2}} \frac{\partial \Theta}{\partial X^{i}} \mathbf{g}^{i}=-\frac{\operatorname{Grad} \Theta}{\Theta^{2}} .
$$


By multiplying Eq. (3.2.56) by $-\Theta$, we obtain the entropy inequality for the species $i$ :

$$
\rightarrow \quad-\Theta \rho_{0}^{i} \frac{d N^{i}}{d t} \leq-\rho_{0}^{i} R^{i}-\Theta \rho_{0}^{i} \tilde{\mathcal{N}}^{i}+\operatorname{Div} \mathbf{Q}^{i}-\frac{\operatorname{Grad} \Theta \cdot \mathbf{Q}^{i}}{\Theta}+\Theta \boldsymbol{M}^{i} \cdot \operatorname{Grad} N^{i}
$$

Summing up over $i$ and adding Eq. (3.2.48) gives the entropy inequality for the whole system

$$
\begin{aligned}
& \sum_{i} \rho_{0}^{i}\left(-\Theta \frac{d N^{i}}{d t}+\frac{d E^{i}}{d t}\right) \leq \sum_{i}\left(-\rho_{0}^{i} R^{i}-\Theta \rho_{0}^{i} \tilde{\mathcal{N}}^{i}+\operatorname{Div} \mathbf{Q}^{i}-\frac{\operatorname{Grad} \Theta \cdot \mathbf{Q}^{i}}{\Theta}+\Theta \boldsymbol{M}^{i} \cdot \operatorname{Grad} N^{i}\right) \\
&+\sum_{i}\left[\left(\dot{\mathbf{F}}+\operatorname{Grad} \mathbf{V}^{i}\right): \boldsymbol{P}^{i}+\rho_{0}^{i} R^{i}-\operatorname{Div} \mathbf{Q}^{i}-\operatorname{Grad} E^{i} \cdot \boldsymbol{M}^{i}\right] \\
&-\sum_{i}\left[\Pi^{i}\left(E^{i}+\frac{1}{2}\left(\mathbf{V}^{i}\right)^{2}\right)\right], \\
& \rightarrow \quad 0 \leq \sum_{i} \rho_{0}^{i}\left(\Theta \frac{d N^{i}}{d t}-\frac{d E^{i}}{d t}\right)+\sum_{i}\left(-\Theta \rho_{0}^{i} \tilde{\mathcal{N}}^{i}-\frac{\operatorname{Grad} \Theta \cdot \mathbf{Q}^{i}}{\Theta}+\Theta \boldsymbol{M}^{i} \cdot \operatorname{Grad} N^{i}\right) \\
&+\sum_{i}\left[\left(\dot{\mathbf{F}}+\operatorname{Grad} \mathbf{V}^{i}\right): \boldsymbol{P}^{i}-\operatorname{Grad} E^{i} \cdot \boldsymbol{M}^{i}\right]-\sum_{i}\left[\Pi^{i}\left(E^{i}+\frac{1}{2}\left(\mathbf{V}^{i}\right)^{2}\right)\right]
\end{aligned}
$$

After rearrangement of the inequality

$$
\begin{aligned}
0 \leq & \sum_{i}\left[\rho_{0}^{i}\left(\Theta \frac{d N^{i}}{d t}-\frac{d E^{i}}{d t}\right)+\left(\dot{\mathbf{F}}+\operatorname{Grad} \mathbf{V}^{i}\right): \boldsymbol{P}^{i}-\frac{\operatorname{Grad} \Theta \cdot \mathbf{Q}^{i}}{\Theta}\right] \\
& +\sum_{i}\left[\left(\Theta \operatorname{Grad} N^{i}-\operatorname{Grad} E^{i}\right) \cdot \boldsymbol{M}^{i}-\Pi^{i}\left(E^{i}+\frac{1}{2}\left(\mathbf{V}^{i}\right)^{2}\right)-\Theta \rho_{0}^{i} \tilde{\mathcal{N}}^{i}\right],
\end{aligned}
$$

and with the following definition of the free energy density $\Psi$

$$
\Psi \stackrel{\text { def }}{=} E-\Theta N \quad \rightarrow \quad \dot{\Psi}+\dot{\Theta} N=\dot{E}-\Theta \dot{N}
$$

and the assumption that the total entropy transfer inside the body is zero,

$$
\sum_{i}\left(\rho_{0}^{i} \tilde{\mathcal{N}}^{i}\right)=0
$$

we obtain the dissipation inequality:

$0 \leq \sum_{i} \mathcal{D}_{\text {closed }}-\sum_{i} \mathcal{G}_{\text {open }}-\sum_{i} \mathcal{G}_{\text {transfer }}=\sum_{i} \mathcal{D}_{\text {closed }}-\sum_{i} \mathcal{G}_{\text {flux }}-\sum_{i} \mathcal{G}_{\text {source }}$ 
whereby

$$
\begin{aligned}
\mathcal{D}_{\text {closed }} & \stackrel{\text { def }}{=}-\rho_{0}^{i}(\dot{\Psi}+\dot{\Theta} N)+\left(\dot{\mathbf{F}}+\operatorname{Grad} \mathbf{V}^{i}\right): P^{i}-\frac{\operatorname{Grad} \Theta \cdot \mathbf{Q}^{i}}{\Theta} \\
\mathcal{G}_{\text {open }} & \stackrel{\text { def }}{=} \mathcal{G}_{\text {flux }}+\mathcal{G}_{\text {source }} \\
\mathcal{G}_{\text {flux }} & \stackrel{\text { def }}{=}\left(\operatorname{Grad} E^{i}-\Theta \operatorname{Grad} N^{i}\right) \cdot \boldsymbol{M}^{i} \\
\mathcal{G}_{\text {source }} & \stackrel{\text { def }}{=} \Pi^{i}\left(E^{i}+\frac{1}{2}\left(\mathbf{V}^{i}\right)^{2}\right) \\
\mathcal{G}_{\text {transfer }} & \stackrel{\text { def }}{=} \Theta \rho_{0}^{i} \tilde{\mathcal{N}}^{i}, \quad \sum \mathcal{G}_{\text {transfer }}=0
\end{aligned}
$$

We can see the dissipation $\mathcal{D}_{\text {closed }}$ in Eq. (3.2.62a) that is the familiar expression from classical continuum mechanics. The mass source and the mass flux cause the entropy change whose contributions are expressed in Eq. (3.2.62c) and (3.2.62d).

Moreover, we can say about the non-negative contributions of the mass source to the dissipation that

$$
0 \leq \mathcal{G}_{\text {source }}
$$

If mass source exists and mass flux does not, the dissipation of the system $\sum_{i} \mathcal{D}_{\text {closed }}$ is at least bigger than the entropy contribution of mass source $\sum_{i} \mathcal{G}_{\text {source }}$.

\subsection{Reduction from an open system to a closed system}

If a system has no mass change, the system becomes a closed one, and the balance equations should be reduced to those of a closed system.

The conditions of no mass change are

$$
\Pi^{i}=0 \quad \text { and } \quad \boldsymbol{M}^{i}=\boldsymbol{O}, \quad \forall i=\mathrm{S}, \mathrm{F}, \mathrm{N}, \mathrm{B}
$$

The balance of mass Eq. (3.2.5) becomes

$$
\frac{d \rho_{0}^{i}}{d t}=0
$$

The balance of linear momentum Eq. (3.2.19) becomes

$$
\rho_{0}^{i} \frac{d\left(\boldsymbol{V}+\boldsymbol{V}^{i}\right)}{d t}=\rho_{0}^{i} \mathbf{g}+\operatorname{Div} \mathbf{P}^{i} .
$$


The balance of angular momentum Eq. (3.2.33) does not change

$$
\boldsymbol{\sigma}^{i}=\left(\boldsymbol{\sigma}^{i}\right)^{\mathrm{T}}
$$

The balance of energy Eq. (3.2.45) becomes

$$
\rho_{0}^{i} \frac{d E^{i}}{d t}=\operatorname{Grad}\left(\boldsymbol{V}+\boldsymbol{V}^{i}\right): \mathbf{P}^{i}+\rho_{0}^{i} R^{i}-\operatorname{Div} \mathbf{Q}^{i}+\rho_{0}^{i} \tilde{E}^{i} .
$$

The entropy inequality (3.2.57) becomes

$$
-\Theta \rho_{0}^{i} \frac{d N^{i}}{d t} \leq-\rho_{0}^{i} R^{i}-\Theta \rho_{0}^{i} \tilde{\mathcal{N}}^{i}+\operatorname{Div} \mathbf{Q}^{i}-\frac{\operatorname{Grad} \Theta \cdot \mathbf{Q}^{i}}{\Theta} .
$$

If the system is composed of just one material, Eqs. (3.3.2a)-(3.3.2e) can be more simplified to

$$
\begin{aligned}
\frac{d \rho_{0}}{d t} & =0 \\
\rho_{0} \frac{d \boldsymbol{V}}{d t} & =\rho_{0} \mathbf{g}+\operatorname{Div} \mathbf{P} \\
\boldsymbol{\sigma} & =\boldsymbol{\sigma}^{\mathrm{T}}, \\
\rho_{0} \frac{d E}{d t} & =\operatorname{Grad} \boldsymbol{V}: \mathbf{P}+\rho_{0} R-\operatorname{Div} \mathbf{Q} \quad \text { and } \\
-\Theta \rho_{0} \frac{d N}{d t} & \leq-\rho_{0} R+\operatorname{Div} \mathbf{Q}-\frac{\operatorname{Grad} \Theta \cdot \mathbf{Q}}{\Theta} .
\end{aligned}
$$

where the energy and entropy transfer from other species, $\tilde{E}^{i}$ and $\tilde{\mathcal{N}}^{i}$ respectively, are zero. These are the same as Eqs. (B.2.3a), ․, (B.2.3e) in Appendix B.2.

As expected, the open system considered in Chapter 3 includes the closed system given by Eqs. (3.3.2a)-(3.3.2e) which can be regarded as a special case of the open system whose conditions are Eqs. (3.3.1). 


\section{Chapter 4}

\section{Constitutive modeling of soft tissue}

The present chapter deals with continuum mechanical models of soft tissue.

In Section 4.1 hydrated soft tissue is discussed with considering its solid/fluid phase and fluid motion through solid. Section 4.2 deals with general growth and remodeling phenomena of a biological system with respect to biomechanics. At the end Section 4.3, we propose a biomechanical model of a cell-seeded soft tissue that has a potential as an articular cartilage replacement material.

\subsection{Mechanical behaviors of soft tissue}

As mentioned in Sec. 2.3, articular cartilage is a solid-fluid mixture. In the present section, different kinds of soft tissue models are introduced with respect to its solid phase and solid-fluid interaction.

\subsubsection{Solid behavior}

Soft tissue can be regarded as elastic or viscoelastic solid with or without fiber-reinforcement.

\section{Fung-elastic material}

With repeated loading cycles, load-deformation curves of soft tissues shift to the right in a load-elongation diagram and the hysteretic effects diminish, as seen in Fig. 4.1. In a load-time diagram the load-time curves shift upwards with increasing number of repeated loading. Thus a 

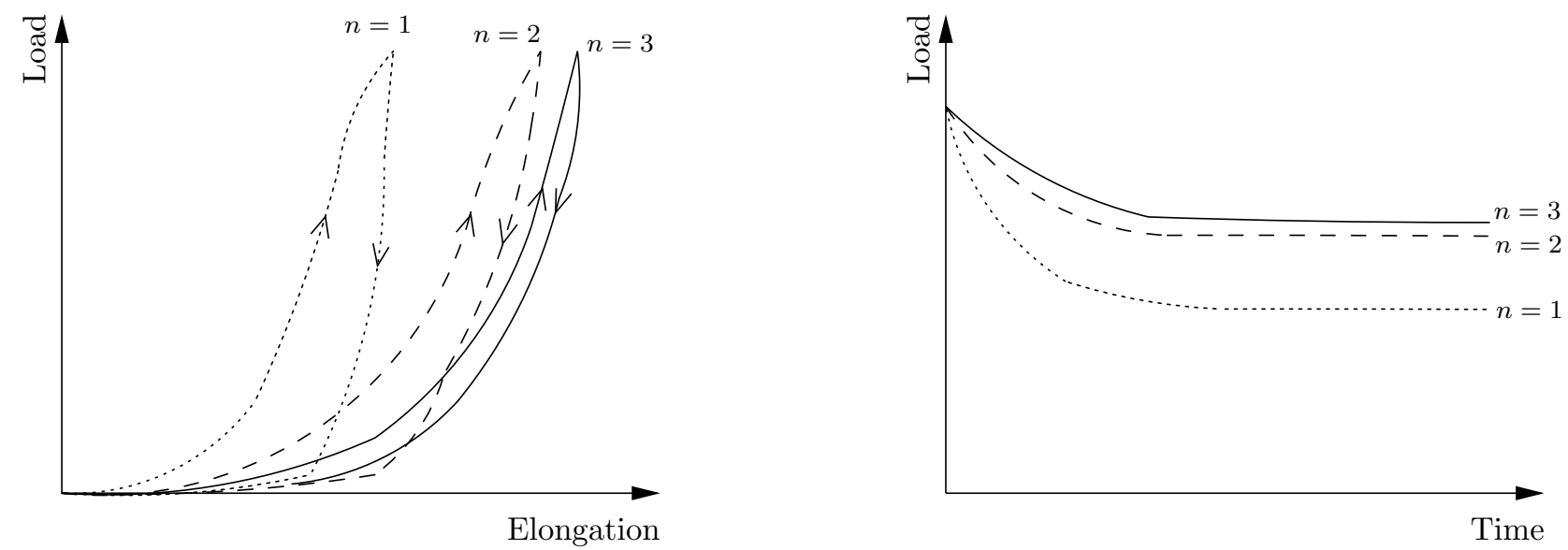

Figure 4.1: Precondition (Modified from Fung [25]).

steady state can be reached, that is so-called precondition. Then, soft tissues can be regarded as independent of strain rate and approximated by a hyperelastic model to a load pattern.

$$
\mathbf{S}=\mathbf{S}(\mathbf{E}, \dot{\mathbf{E}}) \quad \rightarrow \quad \mathbf{S}=\mathbf{S}(\mathbf{E})
$$

The preconditioned soft tissues still present hysteresis, so the mechanical response can be modeled as hyperelastic with different material constants at loading and unloading. Fung [25] has called this model "pseudoelastic" as such a material is not truly elastic. He also pointed out that the stiffness-stress diagram gives a near linear relationship

$$
\frac{d P_{11}}{d \lambda}=\alpha+\beta P_{11}
$$

where $P_{11}$ is the uniaxial first Piola-Kirchhoff stress and $\lambda$ a stretch ration in its direction, $\alpha$ and $\beta$ material parameters. This first-order ordinary differential equation suggests an exponential stress-stretch relation. Fung suggested a refined theory of an exponential stress-strain behavior

$$
W=c[\exp (Q)-1] \quad \rightarrow \quad \mathbf{S}=\frac{\partial W}{\partial \mathbf{E}}=c \exp (Q) \frac{\partial Q}{\partial \mathbf{E}}
$$

where $W$ is a three-dimensional pseudostrain energy function, $c$ a material parameter, $Q$ a function of Green strain tensor E defined in (B.1.24). Fung considered various functional forms for $Q$ by fitting to experimental data. One of them is a quadratic form,

$$
Q=\frac{1}{2} \mathbf{E}: \mathbb{C}: \mathbf{E}
$$


where $\mathbb{C}$ is a fourth-order material tensor. He modified Eq. (4.1.3) taking incompressibility of tissues into consideration

$$
\mathbf{S}=c \frac{\partial \tilde{W}}{\partial \mathbf{E}}-p \mathbf{C}^{-1}=c \exp (Q) \frac{\partial Q}{\partial \mathbf{E}}-p \mathbf{C}^{-1}
$$

where $p$, a Lagrange multiplier, enforces the constraint of incompressibility and $\mathbf{C}$ is the right Cauchy-Green tensor.

Based on Fung's model, Demiray [17] postulated a simple possible form of the strain energy function for isotropic, homogeneous and incompressible elastic soft tissue:

$$
\begin{aligned}
\tilde{W} & =\frac{\beta}{2 \alpha}\left[\exp \left(\alpha\left(I_{1}-3\right)\right)-1\right] \\
\rightarrow \quad \mathbf{S} & =\frac{\beta}{2} \exp \left[\alpha\left(I_{1}-3\right)\right] \mathbf{I}-p \mathbf{C}^{-1} \quad \text { or } \quad \boldsymbol{\sigma}=\frac{\beta}{2} \exp \left[\alpha\left(I_{1}-3\right)\right] \mathbf{b}-p \mathbf{I}
\end{aligned}
$$

where $\mathbf{S}$ and $\boldsymbol{\sigma}$ are the second Piola-Kirchhoff stress tensor and the Cauchy stress tensor respectively and $\mathbf{I}$ the second-order identity tensor. $\alpha$ and $\beta$ are material constants and $I_{1}$ is the first invariant of left Cauchy-Green strain tensor $\mathbf{b}$.

\section{Mooney-Rivlin elastic material}

If the strain energy density function $W$ is a linear combination of two invariants of the left Cauchy-Green deformation tensor $\mathbf{b}$, an incompressible Mooney-Rivlin model has the form

$$
W=C_{1}\left(\lambda_{1}^{2}+\lambda_{2}^{2}+\lambda_{3}^{2}-3\right)+C_{2}\left(\lambda_{1}^{2} \lambda_{2}^{2}+\lambda_{2}^{2} \lambda_{3}^{2}+\lambda_{3}^{2} \lambda_{1}^{2}-3\right) \quad \text { with } \quad \lambda_{1} \lambda_{2} \lambda_{3}=1
$$

where $C_{1}$ and $C_{2}$ are empirically determined material constants and $\lambda_{i}$ is the principal stretch ratios. In the case of uniaxial unconfined loading in $X_{1}$ direction $\left(\sigma_{22}=\sigma_{33}=0\right)$, the Cauchy stress is

$$
\sigma_{11}=\left(2 C_{1}+\frac{2 C_{2}}{\lambda}\right)\left(\lambda^{2}-\frac{1}{\lambda}\right) .
$$

Miller [57] used the strain energy function in order to describe very soft tissues in extension. Miller reduced the energy function to the following forms:

Neo-Hookean material: $\quad W=C_{1}\left(I_{1}-3\right), \quad \mu / 2=C_{1}$

Extreme-Mooney material: $\quad W=C_{2}\left(I_{2}-3\right), \quad \mu / 2=C_{2}$ 
and described the vertical stretch between 1 and 1.3 to high accuracy.

\section{Ogden elastic material}

Ogden material are considered to be isotropic, incompressible and strain rate independent. The strain energy is expressed as

$$
\begin{aligned}
W\left(\lambda_{1}, \lambda_{2}, \lambda_{3}\right) & =\sum_{r=1}^{s} \frac{\mu_{r}}{\alpha_{r}}\left(\lambda_{1}^{\alpha_{r}}+\lambda_{2}^{\alpha_{r}}+\lambda_{3}^{\alpha_{r}}-3\right), \\
2 \mu & =\sum_{r=1}^{s} \mu_{r} \alpha_{r},
\end{aligned}
$$

where $\mu_{r}$ and $\alpha_{r}(r=1 \cdots s)$ represent material constants, $\mu$ shear modulus, and $\lambda_{i}$ the principle stretches. Sayed et al. [72] adopted this kind of strain energy to describe deviatoric elasticity of soft tissues. Oomens et al. [65] employed the model for skin, muscle and fat.

In the case of uniaxial unconfined loading in $X_{1}$ direction $\left(\sigma_{22}=\sigma_{33}=0\right)$, the Cauchy stress is

$$
\sigma_{11}=\sum_{r=1}^{s}\left(\mu_{r} \lambda_{1}^{\alpha_{r}}-\mu_{r} \lambda_{1}^{-1 / 2 \alpha_{r}}\right)
$$

\section{Yeoh elastic material}

The Yeoh model is a reduced polynomial model for nonlinear elastic material and depends on the first and third strain invariants:

$$
W=\sum_{i=1}^{3} C_{i}\left(I_{1}-3\right)^{i}+\sum_{i=1}^{3} \frac{1}{D_{i}}\left(J_{\mathrm{e}}-1\right)^{2 i},
$$

where $C_{i}$ are material constants and $C_{1}$ has the physical meaning of half of the initial shear modulus. The first term explains the behavior of incompressible materials and is reduced to the strain energy density of the incompressible Neo-Hookean material, if $n=1$. The second term is added for a compressible material. $D_{i}$ is a compressibility coefficient that tends to zero for incompressible tissues. Kaster et al. [42] used the model to investigate of hyperelastic properties of brain tissues.

\section{Arruda-Boyce elastic model}

This model describes the behavior of polymer cubic containing eight chains along the diagonal directions.

$$
W=C \sum_{i=1}^{5} \alpha_{i} \lambda^{-2(i-1)}\left(I_{1}^{i}-3^{i}\right)
$$


where $C$ is a material parameter and $\lambda$ the stretch at which the polymer chain network becomes locked. $\alpha_{1}=1 / 2, \alpha_{2}=1 / 20, \alpha_{3}=11 / 1050, \alpha_{4}=19 / 7000$ and $\alpha_{5}=519 / 673750$. Liu et al. [52] applied the strain energy function to model soft tissues undergoing large indentations.

\section{Blatz-Ko elastic model}

Ambrosi and Mollica [7] referred to the Blatz-Ko material to model tumor spheroids

$$
\boldsymbol{\sigma}=\frac{\mu}{J_{\kappa p}}\left[-\left(J_{\kappa p}\right)^{q} \mathbf{I}+\mathbf{F}_{\kappa p} \mathbf{F}_{\kappa p}^{\mathrm{T}}\right]
$$

where $q$ and $\mu$ are material parameters. $\mathbf{F}_{\kappa p}$ is the deformation gradient mapping from a natural configuration to current configuration and $J_{\kappa p}$ is its determinant.

\section{Viscoelastic model}

Due to the hysteresis in stress-strain curve, stress relaxation occurs or creep under constant stress soft tissue is regarded as viscoelastic material. Holzapfel [35] formulated finite strain viscoelasticity. In his work [35], the deformation is decomposed into volumetric elastic and isochoric viscoelastic parts and the following isotropic constitutive model is postulated:

$$
\Psi=\hat{\Psi}\left(\mathbf{C}, \boldsymbol{\Gamma}^{\alpha}\right) \stackrel{\text { def }}{=} U^{\infty}(J)+\tilde{\Psi}^{\infty}(\tilde{\mathbf{C}})+\Upsilon\left(\tilde{\mathbf{C}}, \boldsymbol{\Gamma}^{\alpha}\right), \quad J=\operatorname{det} \mathbf{F}
$$

where $U^{\infty}$ is the volumetric elastic energy, $\tilde{\Psi}^{\infty}$ the isochoric elastic free energy, and $\Upsilon$ is the free energy describing viscoelasticity which depends on internal variables $\Gamma^{\alpha}$ and isochoric strain $\tilde{\mathbf{C}}$. For isothermal process, he derived the second Piola-Kirchhoff stress tensor

$$
\mathbf{S}=\mathbf{S}_{\mathrm{vol}}(J)+\mathbf{S}_{\text {iso }}^{\infty}(\tilde{\mathbf{C}})+\sum_{\alpha=1}^{m} \mathbf{Q}^{\alpha}\left(\tilde{\mathbf{C}}, \Gamma^{\alpha}\right)
$$

where internal variables $\mathbf{Q}^{\alpha}$ are the non-equilibrium stresses and akin to isochoric elastic stress $\mathbf{S}_{\text {iso }}^{\infty}$.

Pioletti and Rakotomanana [67] proposed short time and long time memory for the viscous effect.

$$
\mathbf{S}=\mathbf{S}_{\mathbf{e}}(\mathbf{C}(t))+\mathbf{S}_{\mathrm{v}}(\dot{\mathbf{C}}(t) ; \mathbf{C}(t))+\int_{\delta}^{\infty} \Sigma(\mathbf{G}(t-s), s ; \mathbf{C}(t)) d s,
$$

with $\mathbf{S}$ the second Piola-Kirchhoff stress tensor, $\mathbf{C}$ the right Cauchy-Green strain tensor, $\dot{\mathbf{C}}$ the strain rate tensor and $\mathbf{G}(t-s)=\mathbf{C}(t-s)-\mathbf{C}(t)$. A semicolon is used to separate a parameter 
from variables, i.e. the strain tensor behind the semicolon means that the strain is a parameter. The first term describes the elastic behavior and the second term represents almost immediate contribution, whereas the third term expresses the delayed contribution of long time memory.

\subsubsection{Fiber-reinforced solid behavior}

Destrade et al. [18] incorporated a family of parallel fibers in the simplest elastic material and researched the stability of the resulting solid. They regarded the matrix as an isotropic solid that is reinforced with one family fibers that has a preferred direction

$$
\boldsymbol{N}=\cos \Phi \boldsymbol{E}_{1}+\sin \Phi \boldsymbol{E}_{3}
$$

where $\Phi$ is angle between the fibers and $X_{1}$ direction in reference configuration.

They assumed that the strain energy density is the sum of an isotropic part and an anisotropic part. For the anisotropic part, they take a function of $I_{4}$ only, that is one of the two anisotropic invariants.

$$
\begin{aligned}
W & =\frac{\mu}{2}\left(I_{1}-3\right)+F\left(I_{4}\right) \text { and } I_{4}=1+K \sin 2 \Phi+K^{2} \sin ^{2} \Phi, \\
\boldsymbol{\sigma} & =\mu(\mathbf{b}-\mathbf{I})+2 F^{\prime} \boldsymbol{n} \otimes \boldsymbol{n},
\end{aligned}
$$

where $\mathbf{b}$ is the left Cauchy-Green strain tensor and $\boldsymbol{n}(=\mathbf{F} \boldsymbol{N})$ is the transformed unit vector of $\boldsymbol{N}$ in the current configuration.

Klisch and Lotz as well [44] assumed that the anisotropic properties arise as a result fibers. They introduced two families of fibers whose directions are denoted by unit vectors $\boldsymbol{a}_{0}$ and $\boldsymbol{b}_{0}$ in reference configuration:

$$
\boldsymbol{a}_{0}=\sin \Phi \boldsymbol{E}_{2}+\cos \Phi \boldsymbol{E}_{3} \quad \text { and } \quad \boldsymbol{b}_{0}=-\sin \Phi \boldsymbol{E}_{2}+\cos \Phi \boldsymbol{E}_{3}
$$

where $\Phi$ is angle from $X_{3}$ to $\boldsymbol{a}_{0}$ or $\boldsymbol{b}_{0}$ in different directions to each other. They suggested a strain-energy function

$$
W=\tilde{W}\left(I_{1}, I_{2}, I_{3}, I_{8}, I_{9}, I_{10}, I_{11}, \cos ^{2} 2 \Phi\right)
$$

where $I_{i}$ is strain invariants. They suggested a strain energy function in separate exponential form and derived the constitutive equation for Cauchy stress in terms of the left Cauchy-Green 
strain tensor $\mathbf{b}$.

Holzapfel and Ogden [36] suggested strain energy functions of a material reinforced by a one family fibers and two families of fibers, respectively. The strain energy for an isotropic ground substance (indicated by subscript g) that is reinforced by embedded collagen fibers (indicated by subscript $\mathrm{f}$ ) with a preferred direction $\boldsymbol{N}$ is:

$$
\Psi\left(I_{1}, I_{4}\right)=\Psi_{\mathrm{g}}\left(I_{1}\right)+\Psi_{\mathrm{f}}\left(I_{4}\right)=\frac{c}{2}\left(I_{1}-3\right)+\frac{k_{1}}{k_{2}}\left\{\exp \left[k_{2}\left(I_{4}-1\right)^{2}\right]-1\right\}
$$

$c$ and $k_{1}$ are stress-like material parameters and $k_{4}$ a dimensionless material parameter. The Cauchy stress is

$$
\boldsymbol{\sigma}=-p \mathbf{I}+c \mathbf{b}+4 k_{1}\left(I_{4}-1\right) \exp \left[k_{2}\left(I_{4}-1\right)^{2}\right] \boldsymbol{n} \otimes \boldsymbol{n}
$$

where $\mathbf{b}$ is the left Cauchy-Green strain tensor and $\boldsymbol{n}(=\mathbf{F} \boldsymbol{N})$ is the transformed unit vector of $\boldsymbol{N}$ in the current configuration. If the isotropic ground substance is reinforced by two families of fibers, the strain energy function is

$$
\Psi\left(I_{1}, I_{4}, I_{6}\right)=\Psi_{\mathrm{g}}\left(I_{1}\right)+\Psi_{\mathrm{f}}\left(I_{4}, I_{6}\right)=\frac{c}{2}\left(I_{1}-3\right)+\frac{k_{1}}{k_{2}} \sum_{i=4,6}\left\{\exp \left[k_{2}\left(I_{i}-1\right)^{2}\right]-1\right\}
$$

and the corresponding Cauchy stress is

$$
\boldsymbol{\sigma}=-p \mathbf{I}+c \mathbf{b}+\sum_{i=4,6}\left\{2 k_{1}\left(I_{i}-1\right) \exp \left[k_{2}\left(I_{i}-1\right)^{2}\right] \boldsymbol{n}_{i} \otimes \boldsymbol{n}_{i}\right\}
$$

where $\boldsymbol{n}_{4}$ is the same as $\boldsymbol{n}$ defined in Eq. (4.1.16b) and $\boldsymbol{n}_{6}=\boldsymbol{n}^{\prime}$ is the transformed unit vector of $\boldsymbol{N}^{\prime}$ in a preferred direction of the second fiber family.

\subsubsection{Fluid behavior and fluid-solid interaction}

Kuei, Lai, and Mow [55] suggested a biphasic theory (KLM model) of hydrated tissues such as articular cartilage and interverterbral disk. They modeled articular cartilage as a homogeneous mixture of an incompressible, isotropic, linearly elastic solid (denoted by superscript $\mathbf{s}$ ) and an incompressible, inviscid fluid (denoted by superscript $\mathrm{f}$ ). The interaction between the solid and fluid phases is regarded as a determining factor for the mechanical behavior of biphasic tissues. 
Thus, the permeability $k$ is assumed to be related with the diffusive body force $\boldsymbol{\pi}^{i}(i=\mathbf{f}, \mathbf{s})$ which means, how much fluid flows through the pores according to external force.

$$
\boldsymbol{\pi}^{\mathrm{f}}=-\boldsymbol{\pi}^{\mathrm{s}}=K\left(\boldsymbol{v}^{\mathrm{s}}-\boldsymbol{v}^{\mathrm{f}}\right)
$$

Here, $\boldsymbol{\pi}^{\mathrm{f} / \mathrm{s}}$ is the diffusive body force arising from the frictional drag on fluid and solid, respectively. $K\left(=\frac{\mu}{k}, \quad \mu\right.$ dynamic viscosity $)$ is the constant diffusive drag coefficient and $\boldsymbol{v}^{\mathrm{f} / \mathrm{s}}$ is the velocity of fluid phase and solid phase, respectively. The continuity equation and the equations of motion are respectively

$$
\begin{aligned}
& \operatorname{div} \boldsymbol{v}^{\mathrm{f}}+\alpha \operatorname{div} \boldsymbol{v}^{\mathbf{s}}=0, \\
& \operatorname{div} \boldsymbol{\sigma}^{\mathrm{f} / \mathrm{s}}+\boldsymbol{\pi}^{\mathrm{f} / \mathrm{s}}=\boldsymbol{O} .
\end{aligned}
$$

The constitutive equations are given by

$$
\begin{array}{ll}
\text { solid phase: } & \boldsymbol{\sigma}^{\mathbf{s}}=-\alpha p \mathbf{I}+\lambda^{\mathbf{s}} \operatorname{tr} \boldsymbol{\varepsilon}+2 \mu^{\mathbf{s}} \boldsymbol{\varepsilon}, \\
\text { fluid phase: } & \boldsymbol{\sigma}^{\mathrm{f}}=-p \mathbf{I} .
\end{array}
$$

Ricken [68] and Ehlers [20] regarded soft tissues as fluid-saturated porous media and applied the theory of porous media (TPM). The medium is assumed to be composed of solid and fluid denoted by superscript $\mathbf{s}$ and $\mathbf{f}$, respectively. The constituents are averaged over a representative elementary volume occupied by the whole mixture. Each material point $\boldsymbol{X}^{\alpha}(\alpha=\mathbf{s}, \mathbf{f})$ of a constituent in reference configuration follows its own motion $\varphi^{\alpha}\left(\boldsymbol{X}^{\alpha}, t\right)$ and each spatial point $\boldsymbol{x}$ in the current configuration is simultaneously occupied by solid and fluid material points

$$
\boldsymbol{x}=\boldsymbol{\varphi}^{\alpha}\left(\boldsymbol{X}^{\alpha}, t\right) \quad \text { and } \quad \mathbf{F}^{\alpha}=\frac{\partial \boldsymbol{x}}{\partial \boldsymbol{X}^{\alpha}}
$$

where $\mathbf{F}^{\alpha}$ is the deformation gradient. Each constituent has its own velocity field $\dot{\boldsymbol{x}}^{\alpha}$, where $\{\bullet\}^{\alpha}$ denotes the material time derivative of $\{\bullet\}$ corresponding to the individual motion of the constituent $\alpha$.

The volume fractions $n^{\alpha}$ is defined as

$$
n^{\alpha} \stackrel{\text { def }}{=} \frac{d V^{\alpha}}{d V}=\frac{\rho^{\alpha}}{\rho^{\alpha R}}, \quad \sum_{\alpha}^{\mathrm{s,f}} d V^{\alpha}=d V,
$$


and the saturation condition is assumed as

$$
\sum_{\alpha}^{\mathrm{s}, \mathrm{f}} n^{\alpha}=1
$$

The balance of mass and linear momentum are respectively

$$
\begin{aligned}
& \left(\dot{\rho}^{\alpha}\right)^{\alpha}+\rho^{\alpha} \operatorname{div} \dot{\boldsymbol{x}}^{\alpha}=\hat{\rho}^{\alpha}, \quad \sum_{\alpha}^{\mathrm{s}, \mathrm{f}} \hat{\rho}^{\alpha}=0, \\
& \operatorname{div} \boldsymbol{\sigma}^{\alpha}+\rho^{\alpha} \mathbf{b}+\boldsymbol{\pi}^{\alpha}=\rho^{\alpha} \ddot{\boldsymbol{x}}^{\alpha}+\hat{\rho}^{\alpha} \dot{\boldsymbol{x}}^{\alpha}, \quad \sum_{\alpha}^{\mathbf{s , f}} \boldsymbol{\pi}^{\alpha}=\boldsymbol{O},
\end{aligned}
$$

where $\hat{\rho}^{\alpha}$ is the mass supply between the phases, $\boldsymbol{\sigma}^{\alpha}$ the partial Cauchy stress tensor, $\boldsymbol{\pi}^{\alpha}$ interaction forces, and $\rho^{\alpha} \mathbf{b}$ volume forces.

They assumed the partial Cauchy stress tensors

$$
\boldsymbol{\sigma}^{\alpha}=-n^{\alpha} p \mathbf{I}+\boldsymbol{\sigma}_{E}^{\alpha}, \quad \text { and } \quad \boldsymbol{\pi}^{\mathrm{f}}=p \operatorname{grad} n^{\mathrm{f}}+\boldsymbol{\pi}_{E}^{\mathrm{f}},
$$

where $p$ is the effective fluid pressure and $\boldsymbol{\sigma}_{E}^{\alpha}$ the effective stress governed by the solid deformation (usually $\boldsymbol{\sigma}_{E}^{\mathrm{f}} \approx 0$ ). $\boldsymbol{\pi}_{E}^{\mathrm{f}}$ is an interaction force due to the movement of fluid (friction between the solid and fluid).

Oomens and Campen [66] considered skin as a mixture of solid and fluid. The mixture assumptions and the balance of mass are the same as above: (4.1.21a), (4.1.21b), (4.1.21c) and (4.1.21d). The balance of linear momentum is

$$
\operatorname{div} \boldsymbol{\sigma}^{\alpha}+\rho^{\alpha} \mathbf{b}+\boldsymbol{\pi}^{\alpha}=\rho^{\alpha} \ddot{\boldsymbol{x}}^{\alpha}, \quad \sum_{\alpha}^{\mathrm{s}, \mathrm{f}} \boldsymbol{\pi}^{\alpha}=\boldsymbol{O},
$$

where the mass exchange between solid and fluid $\hat{\rho}^{\alpha}$ in Eq. (4.1.21e) is not considered.

They deduced the following restrictions on the constitutive equations

$$
\begin{aligned}
& \boldsymbol{\sigma}^{\mathbf{s}}=\rho^{\mathbf{s}} \frac{\partial \Psi^{\mathbf{s}}}{\partial \mathbf{F}}\left(\mathbf{F}^{\mathbf{s}}\right)^{\mathrm{T}}-n^{\mathbf{s}} p \mathbf{I}+\rho^{\mathbf{s}} \boldsymbol{v} \frac{\partial \Psi^{\mathbf{s}}}{\partial \boldsymbol{v}}, \\
& \boldsymbol{\sigma}^{\mathrm{f}}=-n^{\mathrm{f}} p \mathbf{I}-\left(\rho^{\mathrm{f}}\right)^{2} \frac{\partial \Psi^{\mathrm{f}}}{\partial \rho^{\mathrm{f}}} \mathbf{I}-\rho^{\mathrm{f}} \rho^{\mathbf{s}} \frac{\partial \Psi^{\mathbf{s}}}{\partial \rho^{\mathrm{f}}} \mathbf{I}-\rho^{\mathbf{s}} \boldsymbol{v} \frac{\partial \Psi^{\mathbf{s}}}{\partial \boldsymbol{v}}, \\
& \Psi^{\mathbf{s}}=\Psi^{\mathbf{s}}\left(\mathbf{F}^{\mathbf{s}}, \rho^{\mathbf{s}}, \boldsymbol{v}\right), \quad \Psi^{\mathrm{f}}=\Psi^{\mathrm{f}}\left(\rho^{\mathrm{f}}, \boldsymbol{v}\right) \quad \text { with } \quad \rho^{\mathbf{s}} \frac{\partial \Psi^{\mathbf{s}}}{\partial \boldsymbol{v}}+\rho^{\mathrm{f}} \frac{\partial \Psi^{\mathrm{f}}}{\partial \boldsymbol{v}}=\boldsymbol{O}, \\
& \boldsymbol{\pi}^{\mathbf{s}} \cdot \boldsymbol{v}+\left(\rho^{\mathbf{s}} \frac{\partial \Psi^{\mathbf{s}}}{\partial \rho^{\mathbf{f}}}+\frac{p}{\rho^{\mathrm{f} R}}\right)\left(\operatorname{grad} \rho^{\mathrm{f}}\right) \cdot \boldsymbol{v} \geq 0,
\end{aligned}
$$

where $\Psi^{\alpha}$ is the Helmholtz free energy, $\boldsymbol{v}\left(\stackrel{\text { def }}{=} \boldsymbol{v}^{\mathrm{f}}-\boldsymbol{v}^{\mathrm{s}}\right)$ the velocity of fluid relative to solid. 


\subsection{Biological behaviors of soft tissue}

The most outstanding features of a biological system are its growth and adaptation to its environment. Biological soft tissues also have the potential to grow and adapt itself to chemical and mechanical stimuli. In a review article [79], Taber distinguishes between growth, remodeling, and morphogenesis.

The phenomenon of growth can be regarded as a process of adding mass such as when hair grows or children grow up. Remodeling processes involve adaptation such as the remodeling of bone by Wolff's laws, stating that a bone will adapt its structure according to the loads it is placed under, and the thickening of the cardiac muscle in response to the growth of blood pressure. Such examples involve changes in material properties. Morphogenesis involves the initial generation of form in developmental biology.

In this section, general phenomena of growth and remodeling will be introduced with models suggested by others, as these are related to the present work.

\subsubsection{Growth}

If the material properties change during the growth, the constitutive relation has the form

$$
\boldsymbol{\sigma}=\boldsymbol{\sigma}\left(\mathbf{C}^{\ell \mathrm{e}}, \mathbf{C}^{\mathrm{g}}\right)
$$

where $\mathbf{C}^{\ell \mathbf{e}}=\left(\mathbf{F}^{\ell} \mathbf{F}^{\mathrm{e}}\right)^{\mathrm{T}} \mathbf{F}^{\ell} \mathbf{F}^{\mathrm{e}}$ and $\mathbf{C}^{\mathrm{g}}=\left(\mathbf{F}^{\mathrm{g}}\right)^{\mathrm{T}} \mathbf{F}^{\mathrm{g}}$.

The residual stress $\boldsymbol{\sigma}(t=0)=\boldsymbol{\sigma}_{0}$ due to volumetric growth and subsequent elastic deformation $\mathbf{F}^{\mathrm{e}} \mathbf{F}^{\mathrm{g}}$ can be obtained by choosing a form for the deformation field ensuring the compatibility of $\mathbf{F}=\mathbf{F}^{\mathrm{e}} \mathbf{F}^{\mathrm{g}}$ with inserting $\mathbf{F}^{\ell}=\mathbf{I}$ in Eq. (3.1.5) and then solving the boundary value problem (traction-free boundary condition). The stress $\boldsymbol{\sigma}(t \neq 0)$ in current configuration can be found with the given total deformation gradient $\mathbf{F}^{\ell} \mathbf{F}^{\mathrm{e}} \mathbf{F}^{\mathrm{g}}$ in Eq. (3.1.5) and boundary conditions (see Fig. 4.2). 


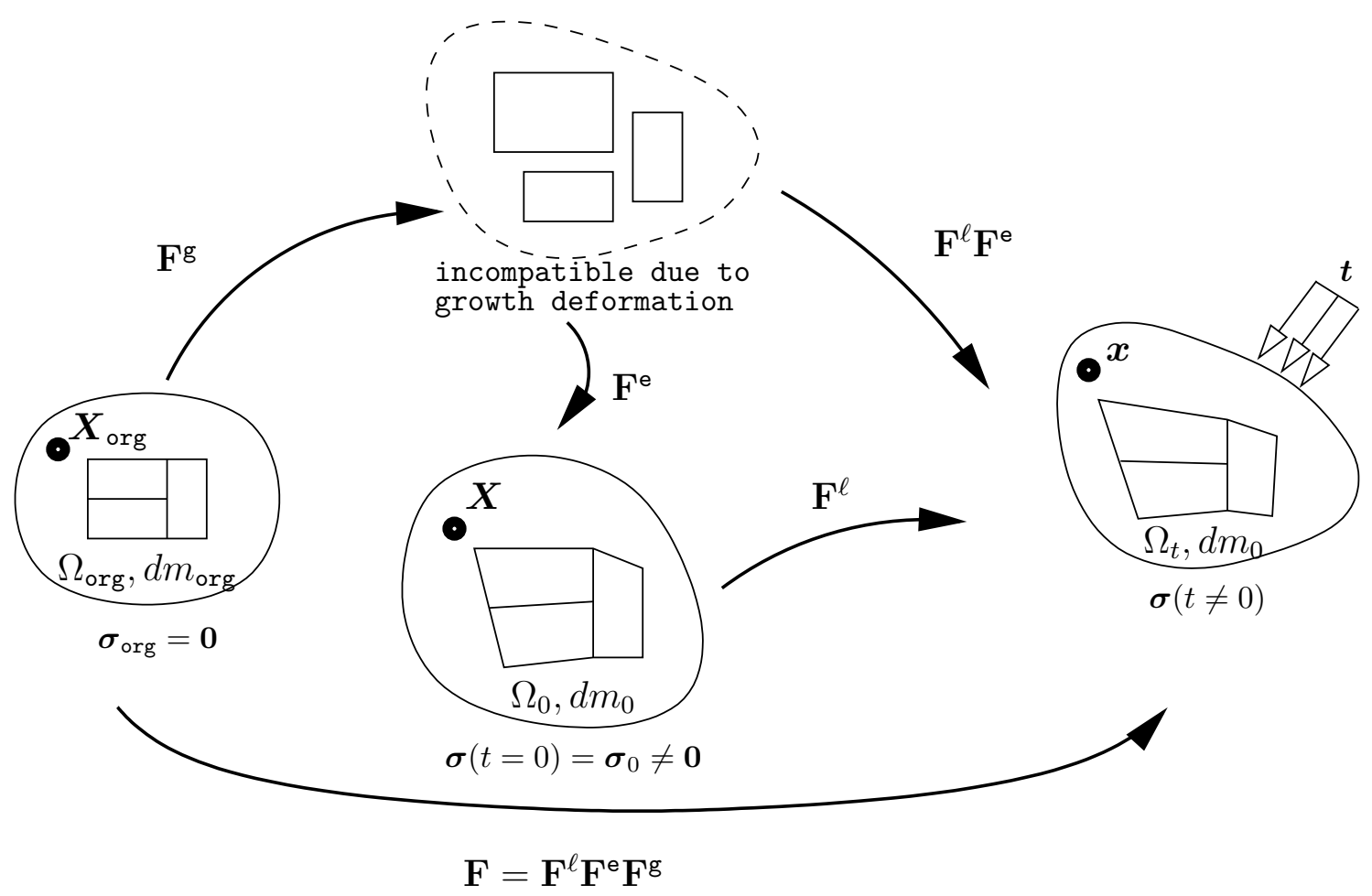

Figure 4.2: Configurations for a growing body (Modified from Rodriguez et al. [41]).

If a growth process is driven by stress, the stress-dependent growth can be expressed with

$$
\dot{\mathbf{F}}^{\mathrm{g}}=\hat{\mathbf{\mathrm { F }}}^{\mathrm{g}}(\boldsymbol{\sigma}),
$$

where a superposed dot denotes partial differentiation with respect to time $t$.

Fung suggested a growth equilibrium stress state $\boldsymbol{\sigma}_{\text {crit }}$ at which the growth rate would be zero $[26]:$

$$
\dot{\mathbf{F}}^{\mathrm{g}}=\hat{\mathbf{F}}^{\mathrm{g}}\left(\boldsymbol{\sigma}-\mathbf{R} \boldsymbol{\sigma}_{\text {crit }} \mathbf{R}^{\mathrm{T}}\right)
$$

where $\mathbf{R}$ is the rotation tensor from the growth equilibrium state to the loaded state. The above equation implies that the growth process has its own criterion that depends on stress.

If $\dot{\mathbf{F}}^{\mathrm{g}}$ and $\mathbf{F}^{\mathrm{g}}$ are known, the rate of growth tensor $\mathbf{D}^{\mathrm{g}}$ can be obtained as (Rodriguez et al., $[41])$

$$
\mathbf{D}^{\mathrm{g}}=\frac{1}{2}\left(\dot{\mathbf{F}}^{\mathrm{g}}\left(\mathbf{F}^{\mathrm{g}}\right)^{-1}+\left(\mathbf{F}^{\mathrm{g}}\right)^{-\mathrm{T}}\left(\dot{\mathbf{F}}^{\mathrm{g}}\right)^{\mathrm{T}}\right)
$$


where dot denotes partial differentiation with respect to time.

If we can set $\mathbf{R}^{\mathrm{g}}=\mathbf{I}$, the growth deformation gradient $\mathbf{F}^{\mathrm{g}}$ becomes growth stretch tensor $\mathbf{U}^{\mathrm{g}}$,

$$
\mathbf{F}^{\mathrm{g}}=\mathbf{R}^{\mathrm{g}} \mathbf{U}^{\mathrm{g}}=\mathbf{U}^{\mathrm{g}},
$$

which is simply computed from rate of growth stretch tensor,

$$
\mathbf{U}^{\mathrm{g}}=\int_{t_{0}}^{t} \dot{\mathbf{U}}^{\mathrm{g}} d t
$$

Then, the rate of growth tensor is given by (Elastic growth models, Goriely et al., [58, Chapter 1])

$$
\mathbf{D}^{\mathrm{g}}=\frac{1}{2}\left(\dot{\mathbf{U}}^{\mathrm{g}}\left(\mathbf{U}^{\mathrm{g}}\right)^{-1}+\left(\mathbf{U}^{\mathrm{g}}\right)^{-1} \dot{\mathbf{U}}^{\mathrm{g}}\right)
$$

The volumetric growth rate $\dot{V}$ can then be computed as ([73])

$$
\frac{1}{V} \frac{d V}{d t}=\operatorname{tr} \mathbf{D}_{\mathrm{g}}
$$

Whereas Fung assumed a critical stress for growth equilibrium, Cowin and van Buskirk [15] introduced a critical strain tensor for growth-equilibrium and described the surface velocity $v$ in the direction of the surface normal $\mathbf{n}$ at position $\boldsymbol{x}$ as

$$
v(\mathbf{n}, \boldsymbol{x}, t)=\mathcal{C}(\mathbf{n}, \boldsymbol{x}):\left[\varepsilon(\boldsymbol{x}, t)-\varepsilon_{\mathrm{crit}}(\boldsymbol{x})\right]=\mathcal{C}_{i j}\left(\varepsilon_{i j}-\varepsilon_{\mathrm{crit} i j}\right)
$$

where $\boldsymbol{\varepsilon}$ is the strain tensor, $\boldsymbol{\varepsilon}_{\text {crit }}$ is strain tensor at growth-equilibrium, and $\mathcal{C}$ is a second-order growth-rate coefficient tensor.

Hart et al. [71] suggested a three-dimensional finite-element model explaining surface growth. They dealt with internal remodeling, and derived the following expression for the rate of change of solid volume fraction $e$ of the material governed by

$$
\dot{e}=a_{0}+a_{1} e+a_{2} e^{2}+\left(\boldsymbol{A}^{0}+\boldsymbol{A}^{1} e\right): \boldsymbol{\varepsilon}=a_{0}+a_{1} e+a_{2} e^{2}+\left(A_{i j}^{0}+A_{i j}^{1} e\right) \varepsilon_{i j},
$$

where $a_{0}, a_{1}, a_{2}, \boldsymbol{A}^{0}$, and $\boldsymbol{A}^{1}$ are constants. With $e$ calculated at a given time step, the local apparent density of a bone tissue is given by

$$
\rho=\gamma\left(e_{0}+e\right)
$$


where $\gamma$ is the true solid density and $e_{0}$ a reference solid volume fraction.

The elastic modulus $E_{i}$ for orthotropic bone is updated according to

$$
E_{i}=c_{i} \rho^{3}
$$

where $c_{i}$ are parameters that are determined by experiments.

\subsubsection{Remodeling}

Cowin and Hegedus [16] regarded bone as a biphasic poroelastic material consisting solid and fluid phases. According to the alterations in loading conditions, the porosity of the bone changes as a consequence of chemical reactions exchanging mass between the two phases. These reactions are controlled by the local strain. Constitutive assumptions for isothermal adaptive elasticity were made on the basis of thermomechanical continuum theory so that

$$
\psi=\tilde{\psi}(\xi, \mathbf{F}), \quad c=\tilde{c}(\xi, \mathbf{F})
$$

where $\psi$ is the specific free-energy density satisfying $\frac{\partial \tilde{\psi}}{\partial \mathbf{F}}(\xi, \mathbf{I})=\mathbf{0}, c$ is the rate at which mass per unit volume is added or removed from the porous matrix structure, $\xi$ denotes the volume fraction of the matrix material in an unstrained reference state, and $\mathbf{F}$ is the deformation gradient.

In [32], $e$ was introduced as a measure of the change in solid volume fraction from a reference volume fraction $\xi_{0}$ as

$$
e=\xi-\xi_{0}
$$

and the following constitutive equations were derived for the case of small strains:

$$
\begin{aligned}
\boldsymbol{\sigma} & =\left(\xi_{0}+e\right) \mathbb{C}: \boldsymbol{\varepsilon}, \\
\dot{e} & =a(e)+\boldsymbol{A}(e): \boldsymbol{\varepsilon},
\end{aligned}
$$

where $\boldsymbol{\sigma}$ is the Cauchy stress, $\boldsymbol{\varepsilon}$ the strain tensor, $\mathbb{C}$ the stiffness tensor (elasticity tensor), and $a$ and $\boldsymbol{A}$ are respective scalar-valued and tensor-valued remodeling rate coefficients.

For small $e$, the above equations can be simplified as

$$
\begin{aligned}
\boldsymbol{\sigma} & =\left(\xi_{0} \mathbb{C}^{0}+e \mathbb{C}^{1}\right): \boldsymbol{\varepsilon}, \\
\dot{e} & =a_{0}+a_{1} e+a_{2} e^{2}+\left(\boldsymbol{A}^{0}+\boldsymbol{A}^{1} e\right): \boldsymbol{\varepsilon},
\end{aligned}
$$


where $\mathbb{C}^{0,1}, \boldsymbol{A}^{0,1}, a_{0}, a_{1}$, and $a_{2}$ are constants. When $\xi_{0}=1$ and $e=0,(4.2 .16 \mathrm{a})$ and (4.2.16b) reduce to Hooke's law $\boldsymbol{\sigma}=\mathbb{C}^{0}: \boldsymbol{\varepsilon}$ with no remodeling.

Cowin and associates separated internal and external remodeling. The internal remodeling is adaptation of elastic modulus and the external remodeling is defined as surface change (shape adaptation):

$$
\begin{aligned}
& \dot{E}=\boldsymbol{A}:\left(\varepsilon-\varepsilon_{\text {crit }}\right), \\
& \dot{X}=\boldsymbol{B}:\left(\varepsilon-\varepsilon_{\text {crit }}\right),
\end{aligned}
$$

where $E$ and $X$ are a local modulus of elasticity and a characteristic surface coordinate perpendicular to the surface, respectively. They have their own remodeling criteria and are assumed to be an equilibrium strain tensor $\varepsilon_{\text {crit }} . \boldsymbol{A}$ and $\boldsymbol{B}$ are second order tensors of internal and external remodeling, respectively.

Huiskes et al. [37] used the strain energy density $U=\frac{1}{2} \boldsymbol{\sigma}: \boldsymbol{\varepsilon}$ as a feed-back control variable. $\boldsymbol{\sigma}$ and $\varepsilon$ are the local stress and strain tensor.

$$
\begin{aligned}
& \dot{E}=C_{e}\left(U-U_{\text {crit }}\right), \\
& \dot{X}=C_{x}\left(U-U_{\text {crit }}\right),
\end{aligned}
$$

where $C_{e}$ and $C_{x}$ are scalar-valued internal and external remodeling coefficient, respectively. $U_{\text {crit }}$ is a site-specific homeostatic equilibrium strain energy density. Huiskes and associates introduced further a concept of threshold, i.e. the adaptive activity becomes lazy in some region of $U$. The remodeling can take place in positive (gain) or negative (loss) direction according to $U$.

\section{Isotropic remodeling}

Beaupré et al. [12] introduced tissue stress stimulus $\psi$ and tissue-level stimulus error $e$ :

$$
\begin{aligned}
& \psi=\left(\sum_{i=1}^{N} n_{i} \bar{\sigma}_{i}^{m}\right)^{1 / m} \\
& e=\psi-\psi_{\mathrm{AS}}
\end{aligned}
$$


where $N$ is number of load cases, $n_{i}$ number of cycles per day for the load case $i, \bar{\sigma}_{i}^{m}$ true stress, $m$ empirical weighting exponent, and $\psi_{\mathrm{AB}}$ the stress stimulus of attractor state that is determined experimentally.

The apposition/resorption rate and of the density $\dot{\rho}$ is a piecewise linear function of $\psi$ and has a dead/lazy zone where no density change takes place:

$$
\dot{\rho}= \begin{cases}c(e+w) & \text { for } e<-w \quad \text { (reposition), } \\ 0 & \text { for }-w \leq e \leq w \quad \text { (dead zone) } \\ c(e-w) & \text { for } w<e \quad \text { (apposition) }\end{cases}
$$

where $c$ is a remodeling constant obtained via experiment and $w$ is the half-width of normal activity region (dead zone), Fig. 4.3.

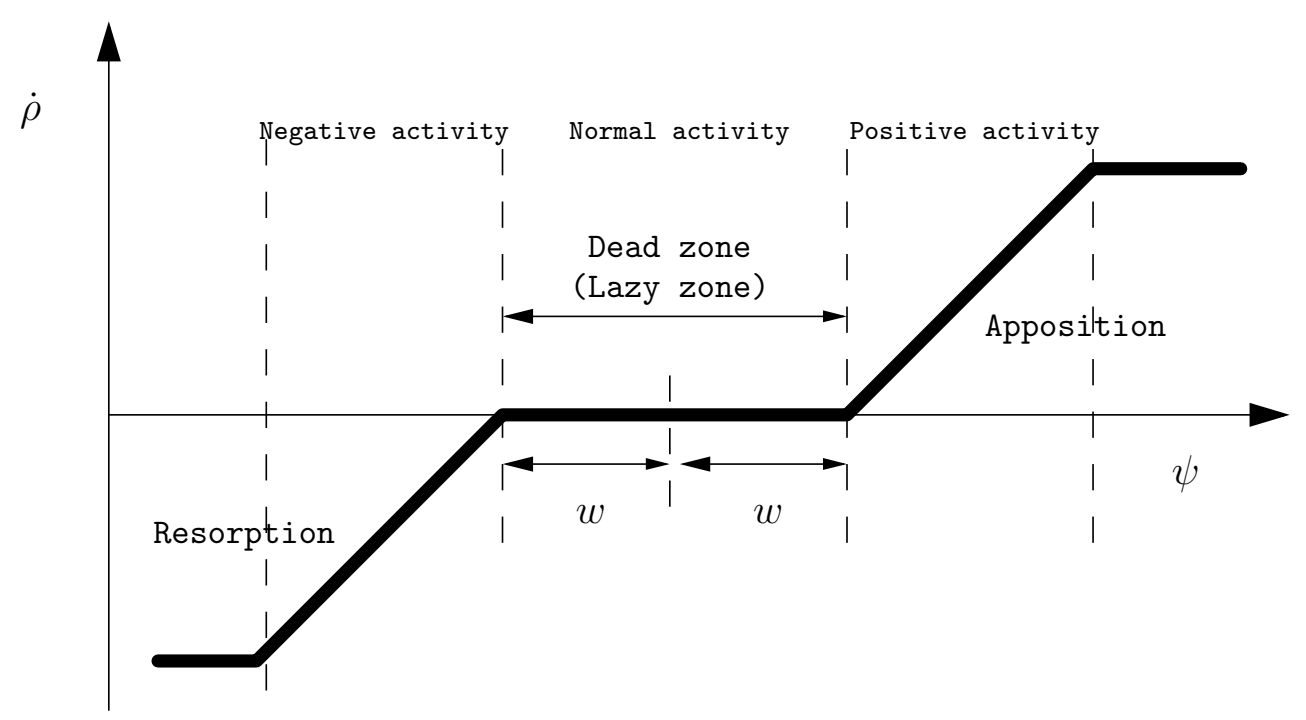

Figure 4.3: Adaptation rate depending on stimulus (Modified from Beaupré et al. [11]).

The elastic modulus $E$ can be expressed as a function of the density $\rho$

$$
E=b \rho^{\beta}
$$

Here, $b$ and $\beta$ are remodeling constants.

\section{Anisotropic remodeling}

Jacobs et al. [40] proposed a daily remodeling stimulus $\psi$ :

$$
\psi=K \frac{\sqrt{\varepsilon: \mathbb{A}: \varepsilon}}{\rho^{2-\beta / 2}}
$$


where $K$ is an empirical function of several constants, $\beta$ the exponent of the isotropic material model, $\mathbb{A}$ the material tensor, and $\varepsilon$ is the strain tensor.

$$
\dot{\mathbb{A}}=\left\{\begin{array}{cl}
-\gamma^{\mathrm{r}} \frac{\boldsymbol{\sigma} \otimes \boldsymbol{\sigma}}{\boldsymbol{\sigma}: \boldsymbol{\varepsilon}} & (\text { net resorption) } \\
\mathbb{0} & (\text { dead zone) } \\
\gamma^{\mathrm{a}} \frac{\boldsymbol{\sigma} \otimes \boldsymbol{\sigma}}{\boldsymbol{\sigma}: \boldsymbol{\varepsilon}} & \text { (net apposition) }
\end{array}\right.
$$

where $\boldsymbol{\sigma}$ is the Cauchy stress tensor and $\gamma^{\mathrm{r}}$ and $\gamma^{\mathrm{a}}$ are Lagrange multipliers of net resorption and net apposition, respectively. They proposed the density change:

$$
\dot{\rho}= \begin{cases}c(e+w) S_{v}(\rho) & \text { for } e<-w \quad \text { (reposition) } \\ 0 & \text { for }-w \leq e \leq w \quad \text { (dead zone) } \\ c(w-e) S_{v}(\rho) & \text { for } w<e \quad \text { (apposition) }\end{cases}
$$

where $c$ is a remodeling constant obtained via experiment and $w$ is the half-width of normal activity region (dead zone), $e$ is the tissue-level stimulus error defined in Eq. (4.2.19b), and $S_{v}(\rho)$ the surface area per unit tissue volume. 


\subsection{Proposed model: diffusive-remodeled materials}

The change of mass due to the synthesis of collagen type II is assumed to be negligible, as the solid fraction is less than $10 \%$ of the whole mass and the synthesized mass (transition from nutrition to solid) is still less than the solid part of the support material. Additionally, the change of fluid fraction is difficult to consider, because there is no boundary between the fluid inside and outside of a specimen. Therefore, we confine ourselves to a closed system with the assumption of no mass change during the remodeling process in a bioreactor.

Due to the fact that the cell-seeded collagen type II material is composed of fluid and solid, solid phase and fluid phase should be separately modeled. However, in order to provide a practical material parameter identification procedure, the following constitutive model is developed with the assumption of a monophasic material with solid behavior. The fluid behavior in the solid phase is taken into account with an evolution equation.

\subsubsection{Assumptions}

\section{Thermodynamic aspect}

Concerning the thermal properties of biological soft tissue, the fluid content of the tissue plays an important role. Like other soft biological tissues the cell-seeded condensed collagen gel contains much fluid. Thus, it is assumed that the material is highly thermally conductive and every point of the system has the same temparature $\Theta$ :

$$
\operatorname{Grad} \Theta=\boldsymbol{O}
$$

Proving that the temperature of the system remains constant, the process can be regarded as an isothermal process, Subsection 5.2.1:

$$
\dot{\Theta}=0
$$

Therefore, the Clausius-Duhem inequality (B.2.3e) in Appendix B.2 can be simplified:

$$
-\rho_{0} \dot{\Psi}+\mathbf{S}: \mathbf{D} \geq 0
$$

Using the identity $\mathbf{D}=\frac{1}{2} \dot{\mathbf{C}}$ the dissipation of the energy $\mathcal{D}$ from the Clausius-Duhem inequality 


$$
\mathcal{D}=\mathbf{S}: \frac{1}{2} \dot{\mathbf{C}}-\rho_{0} \dot{\Psi} \geq 0 .
$$

If the material is purely elastic as a special case, then the free energy (or strain energy in this case) depends only on the deformation:

$$
\Psi=\hat{\Psi}(\mathbf{C}) \quad \rightarrow \quad \dot{\Psi}=\frac{\partial \Psi}{\partial \mathbf{C}}: \dot{\mathbf{C}} .
$$

As the dissipation is zero in elastic deformation

$$
\mathcal{D}=\mathbf{S}: \frac{1}{2} \dot{\mathbf{C}}-\rho_{0} \frac{\partial \Psi}{\partial \mathbf{C}}: \dot{\mathbf{C}}=\left(\frac{1}{2} \mathbf{S}-\rho_{0} \frac{\partial \Psi}{\partial \mathbf{C}}\right): \dot{\mathbf{C}}=0,
$$

the second Piola-Kirchhoff stress tensor is obtained by

$$
\mathbf{S}=2 \rho_{0} \frac{\partial \Psi}{\partial \mathbf{C}} .
$$

\section{Mechanical aspect}

We assume that the solid part of the material is viscoelastic. The elasticity tensor and the viscosity tensor of a transversely isotropic solid material are generally derived in the following Section 4.3.2.

The fluid behavior of the material is considered with the concept of diffusion. Fluid diffuses through the pores of the solid part leading to the stress relaxation or creep response by the compression test.

Additionally it is assumed that the elasticity tensor changes due to the newly synthesized collagen type II (remodeling of elasticity tensor), Section 4.3.4.

\subsubsection{Viscoelastic solid model}

In the present Subsection, a general transversely isotropic viscohyperelastic constitutive law is derived following mainly Limbert and Middleton [51], that is applicable to many biological 
fiber-reinforced tissues such as articular cartilage, ligament and artery.

It is assumed that the elastic part of the solid is hyperelastic (Green elastic, a special case of a Cauchy elastic material) and the stress-strain relationship can be derived from a strain energy density function $\Psi^{\mathrm{e}}$.

Due to the additional viscous deformation, the free energy is composed of an elastic part and a viscous part:

$$
\Psi(\mathbf{C}, \dot{\mathbf{C}})=\Psi^{\mathrm{e}}(\mathbf{C})+\Psi^{\mathrm{v}}(\mathbf{C}, \dot{\mathbf{C}})
$$

The internal dissipation (or local entropy production) [51] is

$$
\mathcal{D}_{\text {int }}=\mathbf{S}: \frac{1}{2} \dot{\mathbf{C}}-\rho_{0} \dot{\Psi}^{\mathrm{e}} \geq 0 \text {. }
$$

The derivation of $\Psi^{\mathrm{e}}$ with respect to time becomes

$$
\mathcal{D}_{\text {int }}=\mathbf{S}: \frac{1}{2} \dot{\mathbf{C}}-\rho_{0} \frac{\partial \Psi^{\mathrm{e}}}{\partial \mathbf{C}}: \dot{\mathbf{C}}=\left(\frac{1}{2} \mathbf{S}-\rho_{0} \frac{\partial \Psi^{\mathrm{e}}}{\partial \mathbf{C}}\right): \dot{\mathbf{C}} \geq 0 .
$$

It is assumed that viscous effects produce the local entropy. The derivation of $\Psi^{\mathrm{v}}$ with respect to $\dot{\mathbf{C}}$ reads

$$
\mathcal{D}_{\text {int }}=\rho_{0} \frac{\partial \Psi^{\mathrm{v}}}{\partial \dot{\mathbf{C}}}: \dot{\mathbf{C}} \geq 0
$$

where $\mathbf{C}$ is not a variable, but a parameter in the definition of $\Psi^{\mathrm{v}}$, which means that coupling between elastic and viscous effects can be separated from each other. From the assumption and the above inequality, we obtain

$$
\left(\frac{1}{2} \mathbf{S}-\rho_{0} \frac{\partial \Psi^{\mathrm{e}}}{\partial \mathbf{C}}\right): \dot{\mathbf{C}}=\rho_{0} \frac{\partial \Psi^{\mathrm{v}}}{\partial \dot{\mathbf{C}}}: \dot{\mathbf{C}} \quad \rightarrow \quad \mathbf{S}=2 \rho_{0}\left(\frac{\partial \Psi^{\mathrm{e}}}{\partial \mathbf{C}}+\frac{\partial \Psi^{\mathrm{v}}}{\partial \dot{\mathbf{C}}}\right)
$$

Now we define the elastic and viscous 2nd Piola-Kirchhoff stress tensor:

$$
\mathbf{S}^{\mathrm{e}} \stackrel{\text { def }}{=} 2 \rho_{0} \frac{\partial \Psi^{\mathrm{e}}}{\partial \mathbf{C}} \quad \text { and } \quad \mathbf{S}^{\mathbf{v}} \stackrel{\text { def }}{=} 2 \rho_{0} \frac{\partial \Psi^{\mathrm{v}}}{\partial \dot{\mathbf{C}}} \text {. }
$$

In order to form the irreducible integrity bases of the tensors $\mathbf{C}$ for the elastic behavior, $\dot{\mathbf{C}}$ for viscous behavior, and the structure tensor $\mathbf{N}_{0}\left(\stackrel{\text { def }}{=} \mathbf{n}_{0} \otimes \mathbf{n}_{0}\right.$, for the transversely isotropic response) a set of 17 invariants $\hat{I}_{i}$ are needed, [14]:

$$
\begin{aligned}
\Psi\left(\mathbf{C}, \dot{\mathbf{C}}, \mathbf{N}_{0}\right) & =\hat{\Psi}\left[\hat{I}_{i}\left(\mathbf{C}, \dot{\mathbf{C}}, \mathbf{N}_{0}\right)_{i=1, \cdots 17}\right] \\
& =\hat{\Psi}^{\mathrm{e}}\left[I_{\alpha}\left(\mathbf{C}, \mathbf{N}_{0}\right)_{\alpha=1, \cdots 5}\right]+\hat{\Psi}^{\mathrm{v}}\left[J_{\beta}\left(\mathbf{C}, \dot{\mathbf{C}}, \mathbf{N}_{0}\right)_{\beta=1, \cdots 12}\right]
\end{aligned}
$$


This free energy function is objective as it is described as a function of $\mathbf{C}, \dot{\mathbf{C}}$, and $\mathbf{N}_{0}$, Section 4.3.2.1.

The strain invariants $I_{\alpha}(\alpha=1, \cdots 5)$ are

$$
\begin{aligned}
& I_{1}=\operatorname{tr} \mathbf{C}, \\
& I_{2}=\frac{1}{2}\left[(\operatorname{tr} \mathbf{C})^{2}-\operatorname{tr} \mathbf{C}^{2}\right], \\
& I_{4}=\operatorname{det} \mathbf{C}=\operatorname{det}\left(\mathbf{F}^{\mathrm{T}} \mathbf{F}\right)=\operatorname{det} \mathbf{F}^{\mathrm{T}} \operatorname{det} \mathbf{F}=J^{2}, \\
& I_{5}=\mathbf{N}_{0}: \mathbf{C}^{2}=\left(\mathbf{n}_{0} \otimes \mathbf{n}_{0}\right): \mathbf{C}^{2}=\mathbf{n}_{0} \mathbf{C}^{2} \mathbf{n}_{0},
\end{aligned}
$$

and the invariants of strain rate $J_{\beta}(\beta=1, \cdots 12)$ are

$$
\begin{array}{lll}
J_{1}=\operatorname{tr} \dot{\mathbf{C}}, & J_{2}=\frac{1}{2} \operatorname{tr} \dot{\mathbf{C}}^{2}, & J_{3}=\operatorname{det} \dot{\mathbf{C}}, \\
J_{4}=\mathbf{N}_{0}: \dot{\mathbf{C}}, & J_{5}=\mathbf{N}_{0}: \dot{\mathbf{C}}^{2}, & \\
J_{6}=\operatorname{tr}(\mathbf{C} \dot{\mathbf{C}}), & J_{7}=\operatorname{tr}\left(\mathbf{C}^{2}\right), & J_{8}=\operatorname{tr}\left(\mathbf{C}^{2} \dot{\mathbf{C}}\right), \quad J_{9}=\operatorname{tr}\left(\mathbf{C}^{2} \dot{\mathbf{C}}^{2}\right), \\
J_{10}=\operatorname{tr}\left(\mathbf{N}_{0} \mathbf{C} \dot{\mathbf{C}}\right), & J_{11}=\operatorname{tr}\left(\mathbf{N}_{0} \mathbf{C}^{2}\right), & J_{12}=\operatorname{tr}\left(\mathbf{N}_{0} \mathbf{C}^{2} \dot{\mathbf{C}}\right) .
\end{array}
$$

The invariants $I_{\alpha}(\alpha=1,2,3)$ and $J_{\beta}(\beta=1,2,3,6,7,8,9)$ describe the isotropic response of the material and $I_{\alpha}(\alpha=4,5)$ and $J_{\beta}(\beta=4,5,10,11,12)$ the transversely isotropic response. 


\subsubsection{Objectivity of the constitutive equation}

In order to check the objectivity of the constitutive equation (4.3.12), i.e., to know whether the constitutive equation is invariant under change of frame of reference, the arguments of $\hat{\Psi}\left(\mathbf{C}, \dot{\mathbf{C}}, \mathbf{N}_{0}\right)$ should be analyzed.

Denoting new kinematic quantities registered by a new observer moving and rotating with respect to the previous one by a superscript star, we obtain first for the deformation gradient and its material time derivative:

$$
\begin{aligned}
& \mathbf{F}^{*}=\mathbf{Q F}, \quad \dot{\mathbf{F}}^{*}=\dot{\mathbf{Q}} \mathbf{F}+\mathbf{Q} \dot{\mathbf{F}} \quad \text { and } \\
& \mathbf{C}^{*}=\mathbf{F}^{* \mathrm{~T}} \mathbf{F}^{*}=(\mathbf{Q F})^{\mathrm{T}} \mathbf{Q F}=\mathbf{F}^{\mathrm{T}} \mathbf{Q}^{\mathrm{T}} \mathbf{Q F}=\mathbf{F}^{\mathrm{T}} \mathbf{F}=\mathbf{C},
\end{aligned}
$$

the right Cauchy-Green tensor $\mathbf{C}$ is proved to be invariant.

The material time derivative of the right Cauchy-Green tensor gives

$$
\begin{aligned}
\dot{\mathbf{C}}^{*} & =\left(\mathbf{F}^{* \mathrm{~T}} \mathbf{F}^{*}\right)^{\cdot}=\dot{\mathbf{F}}^{* \mathrm{~T}} \mathbf{F}^{*}+\mathbf{F}^{* \mathrm{~T}} \dot{\mathbf{F}}^{*}=(\dot{\mathbf{Q}} \mathbf{F}+\mathbf{Q} \mathbf{F})^{\mathrm{T}} \mathbf{Q} \mathbf{F}+\mathbf{F}^{\mathrm{T}} \mathbf{Q}^{\mathrm{T}}(\dot{\mathbf{Q}} \mathbf{F}+\mathbf{Q} \dot{\mathbf{F}}) \\
& =\left(\mathbf{F}^{\mathrm{T}} \dot{\mathbf{Q}}^{\mathrm{T}}+\dot{\mathbf{F}}^{\mathrm{T}} \mathbf{Q}^{\mathrm{T}}\right) \mathbf{Q F}+\mathbf{F}^{\mathrm{T}} \mathbf{Q}^{\mathrm{T}}(\dot{\mathbf{Q}} \mathbf{F}+\mathbf{Q} \dot{\mathbf{F}}) \\
& =\mathbf{F}^{\mathrm{T}} \dot{\mathbf{Q}}^{\mathrm{T}} \mathbf{Q} \mathbf{F}+\dot{\mathbf{F}}^{\mathrm{T}} \mathbf{Q}^{\mathrm{T}} \mathbf{Q} \mathbf{F}+\mathbf{F}^{\mathrm{T}} \mathbf{Q}^{\mathrm{T}} \dot{\mathbf{Q}} \mathbf{F}+\mathbf{F}^{\mathrm{T}} \mathbf{Q}^{\mathrm{T}} \mathbf{Q} \dot{\mathbf{F}} \\
& =\mathbf{F}^{\mathrm{T}} \dot{\mathbf{Q}}^{\mathrm{T}} \mathbf{Q} \mathbf{F}+\dot{\mathbf{F}}^{\mathrm{T}} \mathbf{F}+\mathbf{F}^{\mathrm{T}} \mathbf{Q}^{\mathrm{T}} \dot{\mathbf{Q}} \mathbf{F}+\mathbf{F}^{\mathrm{T}} \dot{\mathbf{F}} \\
& =\mathbf{F}^{\mathrm{T}} \dot{\mathbf{Q}}^{\mathrm{T}} \mathbf{Q} \mathbf{F}+\dot{\mathbf{F}}^{\mathrm{T}} \mathbf{F}+\mathbf{F}^{\mathrm{T}} \mathbf{Q}^{\mathrm{T}} \dot{\mathbf{Q}} \mathbf{F}+\mathbf{F}^{\mathrm{T}} \dot{\mathbf{F}} \\
& =\dot{\mathbf{F}}^{\mathrm{T}} \mathbf{F}+\mathbf{F}^{\mathrm{T}} \dot{\mathbf{F}}+\mathbf{F}^{\mathrm{T}}\left(\dot{\mathbf{Q}}^{\mathrm{T}} \mathbf{Q}+\mathbf{Q}^{\mathrm{T}} \dot{\mathbf{Q}}\right) \mathbf{F} \\
& =\dot{\mathbf{F}}^{\mathrm{T}} \mathbf{F}+\mathbf{F}^{\mathrm{T}} \dot{\mathbf{F}}+\mathbf{F}^{\mathrm{T}}\left(\mathbf{Q}^{\mathrm{T}} \mathbf{Q}\right)^{\mathbf{F}} \\
& =\dot{\mathbf{F}}^{\mathrm{T}} \mathbf{F}+\mathbf{F}^{\mathrm{T}} \dot{\mathbf{F}} \\
& =\left(\mathbf{F}^{\mathrm{T}} \mathbf{F}\right)^{\cdot}=\dot{\mathbf{C}}
\end{aligned}
$$

and it is invariant.

The structure tensor $\mathbf{N}_{0}$ shows frame-indifference:

$$
\mathbf{N}_{0}^{*}=\mathbf{n}_{0}^{*} \otimes \mathbf{n}_{0}^{*}=\mathbf{Q n}_{0} \otimes \mathbf{Q n}_{0}=\mathbf{Q n}_{0} \otimes \mathbf{n}_{0} \mathbf{Q}^{\mathrm{T}}=\mathbf{Q N}_{0} \mathbf{Q}^{\mathrm{T}}
$$

The free energy function $\Psi$ is objective, as it is described as a function of $\mathbf{C}, \dot{\mathbf{C}}$, and $\mathbf{N}_{0}$ that are either invariant or frame-indifferent . 


\subsubsection{Elastic stress tensor and viscous stress tensor}

Using the following relations:

$$
\begin{aligned}
\frac{\partial \operatorname{tr} \mathbf{A}}{\partial \mathbf{A}}: \mathbf{X} & =\left.\frac{d}{d t}[\operatorname{tr}(\mathbf{A}+t \mathbf{X})]\right|_{t=0}=\left.\frac{d}{d t}[(\mathbf{A}+t \mathbf{X}): \mathbf{I}]\right|_{t=0}=\left.\frac{d}{d t}(\mathbf{A}+t \mathbf{X})\right|_{t=0}: \mathbf{I} \\
& =\mathbf{X}: \mathbf{I}=\mathbf{I}: \mathbf{X} \rightarrow \frac{\partial \operatorname{tr} \mathbf{A}}{\partial \mathbf{A}}=\mathbf{I} \\
\frac{\partial \operatorname{tr} \mathbf{A}^{2}}{\partial \mathbf{A}}= & \frac{\partial\left(\mathbf{A}: \mathbf{A}^{\mathrm{T}}\right)}{\partial \mathbf{A}}=\frac{\partial(\mathbf{A}: \mathbf{A})}{\partial \mathbf{A}}=2 \mathbf{A}: \mathbf{A}_{, \mathbf{A}}=2 \mathbf{A}: \mathbb{I}=2 \mathbf{A} \\
\frac{\partial \operatorname{tr} \mathbf{A}^{3}}{\partial \mathbf{A}}= & \frac{\partial\left(\mathbf{A}^{2}: \mathbf{A}^{\mathrm{T}}\right)}{\partial \mathbf{A}}=\frac{\partial\left(\mathbf{A}^{2}: \mathbf{A}\right)}{\partial \mathbf{A}}=\mathbf{A}: \mathbf{A}_{, \mathbf{A}}^{2}+\mathbf{A}^{2}: \mathbf{A}_{, \mathbf{A}} \\
= & \mathbf{A}:\left(\mathbf{A}, \mathbf{A} \mathbf{A}+\mathbf{A} \mathbf{A}_{, \mathbf{A}}\right)+\mathbf{A}^{2}: \mathbb{I}=\mathbf{A}:(\mathbb{I} \mathbf{A}+\mathbf{A} \mathbb{I})+\mathbf{A}^{2} \\
= & \mathbf{A}:[(\mathbf{I} \otimes \mathbf{I}) \mathbf{A}+\mathbf{A}(\mathbf{I} \otimes \mathbf{I})]+\mathbf{A}^{2}=\mathbf{A}:[\mathbf{I} \otimes \mathbf{A}+\mathbf{A} \otimes \mathbf{I}]+\mathbf{A}^{2} \\
= & \mathbf{A}: \mathbf{I} \otimes \mathbf{A}+\mathbf{A}: \mathbf{A} \otimes \mathbf{I}+\mathbf{A}^{2}=\mathbf{I}^{\mathrm{T}} \mathbf{A} \mathbf{A}^{\mathrm{T}}+\mathbf{A}^{\mathrm{T}} \mathbf{A} \mathbf{I}^{\mathrm{T}}+\mathbf{A}^{2} \\
= & 3 \mathbf{A}^{2}
\end{aligned}
$$

which can be also derived from the Eq. (A.3.4), the first derivatives of the invariants with respect to $\mathbf{C}$ are obtained:

$$
\begin{aligned}
\frac{\partial I_{1}}{\partial \mathbf{C}} & =\frac{\partial \operatorname{tr} \mathbf{C}}{\partial \mathbf{C}}=\mathbf{I} \\
\frac{\partial I_{2}}{\partial \mathbf{C}} & =\frac{1}{2} \frac{\partial}{\partial \mathbf{C}}\left[(\operatorname{tr} \mathbf{C})^{2}-\operatorname{tr} \mathbf{C}^{2}\right]=\frac{1}{2}\left[2 \operatorname{tr} \mathbf{C} \frac{\partial \operatorname{tr} \mathbf{C}}{\partial \mathbf{C}}-2 \mathbf{C}\right]=\operatorname{tr} \mathbf{C I}-\mathbf{C}=I_{1} \mathbf{I}-\mathbf{C} \\
\frac{\partial I_{3}}{\partial \mathbf{C}} & =\frac{1}{3} \frac{\partial}{\partial \mathbf{C}}\left[\operatorname{tr} \mathbf{C}^{3}-\frac{3}{2} \operatorname{tr} \mathbf{C}^{2} \operatorname{tr} \mathbf{C}+\frac{1}{2}(\operatorname{tr} \mathbf{C})^{3}\right] \\
& =\frac{1}{3}\left[3 \mathbf{C}^{2}-\frac{3}{2}\left(2 \mathbf{C} \operatorname{tr} \mathbf{C}+\operatorname{tr} \mathbf{C}^{2} \mathbf{I}\right)+\frac{3}{2}(\operatorname{tr} \mathbf{C})^{2} \mathbf{I}\right] \\
& =\mathbf{C}^{2}-\mathbf{C} \operatorname{tr} \mathbf{C}-\frac{1}{2} \operatorname{tr} \mathbf{C}^{2} \mathbf{I}+\frac{1}{2}(\operatorname{tr} \mathbf{C})^{2} \mathbf{I}=I_{3} \mathbf{C}^{-1}
\end{aligned}
$$




$$
\begin{aligned}
\frac{\partial I_{4}}{\partial \mathbf{C}} & =\left(\mathbf{N}_{0}: \mathbf{C}\right)_{, \mathbf{C}}=\mathbf{N}_{0}: \mathbf{C}_{, \mathbf{C}}+\mathbf{C}: \mathbf{N}_{0, \mathbf{C}}=\mathbf{N}_{0}: \mathbb{I}+\mathbf{C}: \mathbb{0}=\mathbf{N}_{0}+\mathbf{0}=\mathbf{N}_{0} \\
\frac{\partial I_{5}}{\partial \mathbf{C}} & =\left(\mathbf{N}_{0}: \mathbf{C}^{2}\right)_{, \mathbf{C}}=\mathbf{N}_{0}: \mathbf{C}_{, \mathbf{C}}^{2}+\mathbf{C}^{2}: \mathbf{N}_{0, \mathbf{C}}=\mathbf{N}_{0}:(\mathbb{I} \mathbf{C}+\mathbf{C I})+\mathbf{C}^{2}: \mathbb{0} \\
& =\mathbf{N}_{0}:[(\mathbf{I} \otimes \mathbf{I}) \mathbf{C}+\mathbf{C}(\mathbf{I} \otimes \mathbf{I})]+\mathbf{0}=\mathbf{N}_{0}:[\mathbf{I} \otimes(\mathbf{I C})+(\mathbf{C I}) \otimes \mathbf{I}] \\
& =\mathbf{N}_{0}:[\mathbf{I} \otimes \mathbf{C}+\mathbf{C} \otimes \mathbf{I}]=\mathbf{I}^{\mathrm{T}} \mathbf{N}_{0} \mathbf{C}^{\mathrm{T}}+\mathbf{C}^{\mathrm{T}} \mathbf{N}_{0} \mathbf{I}^{\mathrm{T}}=\mathbf{N}_{0} \mathbf{C}+\mathbf{C N}_{0} \\
& =\mathbf{n}_{0} \otimes \mathbf{n}_{0} \mathbf{C}+\mathbf{C n}_{0} \otimes \mathbf{n}_{0}=\mathbf{n}_{0} \otimes \mathbf{C n}_{0}+\mathbf{n}_{0} \mathbf{C} \otimes \mathbf{n}_{0} \stackrel{\text { def }}{=} \mathbf{\Upsilon}_{\mathbf{n}_{0} \mathbf{C}},
\end{aligned}
$$

where the derivation of the structural tensor $\mathbf{N}_{0, \mathbf{C}}=\mathbb{0}$.

The first derivative of the strain rate invariants with respect to $\dot{\mathbf{C}}$ are

$$
\begin{aligned}
\frac{\partial J_{1}}{\partial \dot{\mathbf{C}}} & =\frac{\partial \operatorname{tr} \dot{\mathbf{C}}}{\partial \dot{\mathbf{C}}}=\mathbf{I} \\
\frac{\partial J_{2}}{\partial \dot{\mathbf{C}}} & =\frac{1}{2} \frac{\partial}{\partial \dot{\mathbf{C}}}\left(\mathbf{I}: \dot{\mathbf{C}}^{2}\right)=\frac{1}{2}\left(\dot{\mathbf{C}}^{2}: \mathbf{I}_{, \dot{\mathbf{C}}}+\mathbf{I}: \dot{\mathbf{C}}_{, \dot{\mathbf{C}}}^{2}\right)=\frac{1}{2}\left(\mathbf{I}: \dot{\mathbf{C}}_{, \dot{\mathbf{C}}}^{2}\right)=\frac{1}{2}[\mathbf{I}:(\mathbf{I} \otimes \dot{\mathbf{C}}+\dot{\mathbf{C}} \otimes \mathbf{I})] \\
& =\frac{1}{2}\left(\mathbf{I}^{\mathrm{T}} \mathbf{I}^{\mathrm{T}}+\dot{\mathbf{C}}^{\mathrm{T}} \mathbf{I I}^{\mathrm{T}}\right)=\dot{\mathbf{C}}^{\mathrm{T}}=\dot{\mathbf{C}} \\
\frac{\partial J_{3}}{\partial \dot{\mathbf{C}}} & =\frac{1}{3} \frac{\partial}{\partial \dot{\mathbf{C}}}\left[\operatorname{tr} \dot{\mathbf{C}}^{3}-\frac{3}{2} \operatorname{tr} \dot{\mathbf{C}}^{2} \operatorname{tr} \dot{\mathbf{C}}+\frac{1}{2}(\operatorname{tr} \dot{\mathbf{C}})^{3}\right] \\
& =\frac{1}{3}\left[3 \dot{\mathbf{C}}^{2}-\frac{3}{2}\left(2 \dot{\mathbf{C}}^{\operatorname{tr}} \dot{\mathbf{C}}+\operatorname{tr} \dot{\mathbf{C}}^{2} \mathbf{I}\right)+\frac{3}{2}(\operatorname{tr} \dot{\mathbf{C}})^{2} \mathbf{I}\right] \\
& =\dot{\mathbf{C}}^{2}-\dot{\mathbf{C}} \operatorname{tr} \dot{\mathbf{C}}-\frac{1}{2} \operatorname{tr} \dot{\mathbf{C}}^{2} \mathbf{I}+\frac{1}{2}(\operatorname{tr} \dot{\mathbf{C}})^{2} \mathbf{I}=J_{3} \dot{\mathbf{C}}^{-1} \\
\frac{\partial J_{4}}{\partial \dot{\mathbf{C}}} & =\left(\mathbf{N}_{0}: \dot{\mathbf{C}}\right)_{, \dot{\mathbf{C}}}=\mathbf{N}_{0}: \dot{\mathbf{C}}_{, \dot{\mathbf{C}}}+\dot{\mathbf{C}}: \mathbf{N}_{0, \dot{\mathbf{C}}}=\mathbf{N}_{0}: \mathbb{I}+\mathbf{0}=\mathbf{N}_{0}
\end{aligned}
$$




$$
\begin{aligned}
\frac{\partial J_{5}}{\partial \dot{\mathbf{C}}} & =\left(\mathbf{N}_{0}: \dot{\mathbf{C}}^{2}\right)_{, \dot{\mathbf{C}}}=\mathbf{N}_{0}: \dot{\mathbf{C}}_{, \dot{\mathbf{C}}}^{2}+\dot{\mathbf{C}}^{2}: \mathbf{N}_{0, \dot{\mathbf{C}}}=\mathbf{N}_{0}:(\mathbf{I} \otimes \dot{\mathbf{C}}+\dot{\mathbf{C}} \otimes \mathbf{I})+\mathbf{0} \\
& =\mathbf{I}^{\mathrm{T}} \mathbf{N}_{0} \dot{\mathbf{C}}^{\mathrm{T}}+\dot{\mathbf{C}}^{\mathrm{T}} \mathbf{N}_{0} \mathbf{I}^{\mathrm{T}}=\mathbf{N}_{0} \dot{\mathbf{C}}+\dot{\mathbf{C}} \mathbf{N}_{0}=\mathbf{n}_{0} \otimes \mathbf{n}_{0} \dot{\mathbf{C}}+\dot{\mathbf{C}} \mathbf{n}_{0} \otimes \mathbf{n}_{0} \\
& =\mathbf{n}_{0} \otimes \dot{\mathbf{C}} \mathbf{n}_{0}+\mathbf{n}_{0} \dot{\mathbf{C}} \otimes \mathbf{n}_{0} \stackrel{\text { def }}{=} \mathbf{\Upsilon}_{\mathbf{n}_{0} \dot{\mathbf{C}}},
\end{aligned}
$$

$$
\begin{aligned}
\frac{\partial J_{6}}{\partial \dot{\mathbf{C}}} & =(\mathbf{I}: \mathbf{C C})_{, \dot{\mathbf{C}}}=\mathbb{O}: \mathbf{C} \dot{\mathbf{C}}+\mathbf{I}:(\mathbf{C} \dot{\mathbf{C}})_{, \dot{\mathbf{C}}}=\mathbf{0}+\mathbf{I}:(\mathbb{D} \dot{\mathbf{C}}+\mathbf{C I})=\mathbf{I}:[\mathbf{C}(\mathbf{I} \otimes \mathbf{I})] \\
& =\mathbf{I}:[(\mathbf{C I}) \otimes \mathbf{I}]=\mathbf{I}:(\mathbf{C} \otimes \mathbf{I})=\mathbf{C}^{\mathrm{T}} \mathbf{I I}^{\mathrm{T}}=\mathbf{C}^{\mathrm{T}}=\mathbf{C},
\end{aligned}
$$$$
\frac{\partial J_{7}}{\partial \dot{\mathbf{C}}}=\left(\mathbf{I}: \mathbf{C} \dot{\mathbf{C}}^{2}\right)_{, \dot{\mathbf{C}}}=\mathbf{C}^{2}: \mathbf{I}_{, \dot{\mathbf{C}}}+\mathbf{I}:\left(\mathbf{C}^{2}\right)_{, \dot{\mathbf{C}}}=\mathbf{C} \dot{\mathbf{C}}^{2}: \mathbb{0}+\mathbf{I}:\left(\mathbf{C}_{, \dot{\mathbf{C}}} \dot{\mathbf{C}}^{2}+\mathbf{C} \dot{\mathbf{C}}_{, \dot{\mathbf{C}}}^{2}\right)
$$$$
=\mathbf{0}+\mathbf{I}:[0 \dot{\mathbf{C}}+\mathbf{C}(\mathbf{I} \otimes \dot{\mathbf{C}}+\dot{\mathbf{C}} \otimes \mathbf{I})]=\mathbf{I}: \mathbf{C}(\mathbf{I} \otimes \dot{\mathbf{C}}+\dot{\mathbf{C}} \otimes \mathbf{I})
$$$$
=\mathbf{I}:(\mathbf{C I} \otimes \dot{\mathbf{C}}+\mathbf{C} \dot{\mathbf{C}} \otimes \mathbf{I})=\mathbf{I}:(\mathbf{C} \otimes \dot{\mathbf{C}}+\mathbf{C} \dot{\mathbf{C}} \otimes \mathbf{I})=\mathbf{I}: \mathbf{C} \otimes \dot{\mathbf{C}}+\mathbf{I}: \mathbf{C} \dot{\mathbf{C}} \otimes \mathbf{I}
$$$$
=\mathbf{C}^{\mathrm{T}} \mathbf{I} \dot{\mathbf{C}}^{\mathrm{T}}+(\mathbf{C} \dot{\mathbf{C}})^{\mathrm{T}} \mathbf{I} \mathbf{I}^{\mathrm{T}}=\mathbf{C}^{\mathrm{T}} \dot{\mathbf{C}}^{\mathrm{T}}+(\mathbf{C} \dot{\mathbf{C}})^{\mathrm{T}}=\mathbf{C} \dot{\mathbf{C}}+\dot{\mathbf{C}} \mathbf{C},
$$

$$
\begin{aligned}
\frac{\partial J_{8}}{\partial \dot{\mathbf{C}}} & =\left(\mathbf{I}: \mathbf{C}^{2} \dot{\mathbf{C}}\right)_{, \dot{\mathbf{C}}}=\mathbf{C}^{2} \dot{\mathbf{C}}: \mathbf{I}_{, \dot{\mathbf{C}}}+\mathbf{I}:\left(\mathbf{C}^{2} \dot{\mathbf{C}}\right)_{, \dot{\mathbf{C}}}=\mathbf{C}^{2} \dot{\mathbf{C}}: \mathbb{0}+\mathbf{I}:\left(\mathbf{C}_{, \dot{\mathbf{C}}}^{2} \dot{\mathbf{C}}+\mathbf{C}^{2} \dot{\mathbf{C}}_{, \dot{\mathbf{C}}}\right) \\
& =\mathbf{0}+\mathbf{I}:\left(\mathbb{0} \dot{\mathbf{C}}+\mathbf{C}^{2} \mathbf{I}\right)=\mathbf{0}+\mathbf{I}: \mathbb{0}+\mathbf{I}: \mathbf{C}^{2} \mathbb{I}=\mathbf{0}+\mathbf{0}+\mathbf{I}: \mathbf{C}^{2} \otimes \mathbf{I} \\
& =\left(\mathbf{C}^{2}\right)^{\mathrm{T}} \mathbf{I I}^{\mathrm{T}}=\mathbf{C}^{2}
\end{aligned}
$$$$
\begin{aligned}
\frac{\partial J_{9}}{\partial \dot{\mathbf{C}}} & =\left(\mathbf{I}: \mathbf{C}^{2} \dot{\mathbf{C}}^{2}\right)_{, \dot{\mathbf{C}}}=\mathbf{C}^{2} \dot{\mathbf{C}}^{2}: \mathbf{I}_{, \dot{\mathbf{C}}}+\mathbf{I}:\left(\mathbf{C}^{2} \dot{\mathbf{C}}^{2}\right)_{, \dot{\mathbf{C}}}=\mathbf{C}^{2} \dot{\mathbf{C}}^{2}: \mathbb{0}+\mathbf{I}:\left(\mathbf{C}_{, \dot{\mathbf{C}}}^{2} \dot{\mathbf{C}}^{2}+\mathbf{C}^{2} \dot{\mathbf{C}}_{, \dot{\mathbf{C}}}^{2}\right) \\
& =\mathbf{0}+\mathbf{I}:\left[\mathbb{0} \dot{\mathbf{C}}^{2}+\mathbf{C}^{2}(\mathbf{I} \otimes \dot{\mathbf{C}}+\dot{\mathbf{C}} \otimes \mathbf{I})\right]=\mathbf{0}+\mathbf{I}:\left(\mathbb{0}+\mathbf{C}^{2} \mathbf{I} \otimes \dot{\mathbf{C}}+\mathbf{C}^{2} \dot{\mathbf{C}} \otimes \mathbf{I}\right) \\
& =\mathbf{0}+\mathbf{I}: \mathbf{C}^{2} \otimes \dot{\mathbf{C}}+\mathbf{I}: \mathbf{C}^{2} \dot{\mathbf{C}} \otimes \mathbf{I}=\mathbf{0}+\left(\mathbf{C}^{2}\right)^{\mathrm{T}} \mathbf{I} \dot{\mathbf{C}}^{\mathrm{T}}+\left(\mathbf{C}^{2} \dot{\mathbf{C}}\right)^{\mathrm{T}} \mathbf{I I}^{\mathrm{T}} \\
& =\mathbf{0}+\mathbf{C}^{2} \mathbf{I} \dot{\mathbf{C}}+\dot{\mathbf{C}}^{\mathrm{T}}\left(\mathbf{C}^{2}\right)^{\mathrm{T}}=\mathbf{C}^{2} \dot{\mathbf{C}}+\dot{\mathbf{C}}^{2},
\end{aligned}
$$ 


$$
\begin{aligned}
\frac{\partial J_{10}}{\partial \dot{\mathbf{C}}} & =\left(\mathbf{I}: \mathbf{N}_{0} \mathbf{C} \dot{\mathbf{C}}\right)_{, \dot{\mathbf{C}}}=\mathbf{N}_{0} \mathbf{C} \dot{\mathbf{C}}: \mathbf{I}_{\dot{\mathbf{C}}}+\mathbf{I}:\left(\mathbf{N}_{0, \dot{\mathbf{C}}} \mathbf{C} \dot{\mathbf{C}}+\mathbf{N}_{0} \mathbf{C}_{, \dot{\mathbf{C}}} \dot{\mathbf{C}}+\mathbf{N}_{0} \mathbf{C} \dot{\mathbf{C}}_{, \dot{\mathbf{C}}}\right) \\
& =\mathbf{0}+\mathbf{I}:\left(\mathbb{0}+\mathbb{0}+\mathbf{N}_{0} \mathbf{C I}\right)=\mathbf{0}+\mathbf{I}: \mathbf{N}_{0} \mathbf{C} \otimes \mathbf{I}=\left(\mathbf{N}_{0} \mathbf{C}\right)^{\mathrm{T}} \mathbf{I I}^{\mathrm{T}} \\
& =\left(\mathbf{N}_{0} \mathbf{C}\right)^{\mathrm{T}}=\mathbf{C}^{\mathrm{T}} \mathbf{N}_{0}^{\mathrm{T}}=\mathbf{C N}_{0}
\end{aligned}
$$$$
\frac{\partial J_{11}}{\partial \dot{\mathbf{C}}}=\left(\mathbf{I}: \mathbf{N}_{0} \mathbf{C} \dot{\mathbf{C}}^{2}\right)_{, \dot{\mathbf{C}}}=\mathbf{I}:\left(\mathbf{N}_{0} \mathbf{C} \dot{\mathbf{C}}_{, \dot{\mathbf{C}}}^{2}\right)=\mathbf{I}: \mathbf{N}_{0} \mathbf{C}(\mathbf{I} \otimes \dot{\mathbf{C}}+\dot{\mathbf{C}} \otimes \mathbf{I})
$$$$
=\mathbf{I}:\left(\mathbf{N}_{0} \mathbf{C I} \otimes \dot{\mathbf{C}}+\mathbf{N}_{0} \mathbf{C} \dot{\mathbf{C}} \otimes \mathbf{I}\right)=\mathbf{I}: \mathbf{N}_{0} \mathbf{C} \otimes \dot{\mathbf{C}}+\mathbf{I}: \mathbf{N}_{0} \mathbf{C} \dot{\mathbf{C}} \otimes \mathbf{I}
$$$$
=\left(\mathbf{N}_{0} \mathbf{C}\right)^{\mathrm{T}} \mathbf{I} \dot{\mathbf{C}}^{\mathrm{T}}+\left(\mathbf{N}_{0} \mathbf{C} \dot{\mathbf{C}}\right)^{\mathrm{T}} \mathbf{I I}^{\mathrm{T}}=\mathbf{C}^{\mathrm{T}} \mathbf{N}_{0}^{\mathrm{T}} \dot{\mathbf{C}}^{\mathrm{T}}+\dot{\mathbf{C}}^{\mathrm{T}} \mathbf{C}^{\mathrm{T}} \mathbf{N}_{0}^{\mathrm{T}}=\mathbf{C N}_{0} \dot{\mathbf{C}}+\dot{\mathbf{C}} \mathbf{C} \mathbf{N}_{0}
$$

$$
\begin{aligned}
\frac{\partial J_{12}}{\partial \dot{\mathbf{C}}} & =\left(\mathbf{I}: \mathbf{N}_{0} \mathbf{C}^{2} \dot{\mathbf{C}}\right)_{, \dot{\mathbf{C}}}=\mathbf{I}:\left(\mathbf{N}_{0} \mathbf{C}^{2} \dot{\mathbf{C}}_{, \dot{\mathbf{C}}}\right)=\mathbf{I}:\left(\mathbf{N}_{0} \mathbf{C}^{2} \mathbf{I}\right)=\mathbf{I}:\left(\mathbf{N}_{0} \mathbf{C}^{2} \otimes \mathbf{I}\right) \\
& =\left(\mathbf{N}_{0} \mathbf{C}^{2}\right)^{\mathrm{T}} \mathbf{I} \mathbf{I}^{\mathrm{T}}=\left(\mathbf{C}^{2}\right)^{\mathrm{T}} \mathbf{N}_{0}^{\mathrm{T}}=\mathbf{C}^{2} \mathbf{N}_{0} .
\end{aligned}
$$


With the following notations

$$
\Psi_{\alpha}^{\mathrm{e}} \stackrel{\text { def }}{=} \frac{\partial \Psi^{\mathrm{e}}}{\partial I_{\alpha}} \quad \text { and } \quad \Psi_{\beta}^{\mathrm{v}} \stackrel{\text { def }}{=} \frac{\partial \Psi^{\mathrm{v}}}{\partial J_{\beta}},
$$

the elastic and viscous 2nd Piola-Kirchhoff stress can be written in a simplified manner.

The elastic 2nd Piola-Kirchhoff stress is

$$
\begin{aligned}
\mathbf{S}^{\mathrm{e}} & =2 \rho_{0} \frac{\partial \Psi^{\mathrm{e}}}{\partial \mathbf{C}}=2 \rho_{0} \sum_{\alpha=1}^{5}\left(\frac{\partial \Psi^{\mathrm{e}}}{\partial I_{\alpha}} \frac{\partial I_{\alpha}}{\partial \mathbf{C}}\right)=2 \rho_{0} \sum_{\alpha=1}^{5}\left(\Psi_{\alpha}^{\mathrm{e}} I_{\alpha, \mathbf{C}}\right) \\
& =2 \rho_{0}\left(\Psi_{1}^{\mathrm{e}} I_{1, \mathbf{C}}+\Psi_{2}^{\mathrm{e}} I_{2, \mathbf{C}}+\Psi_{3}^{\mathrm{e}} I_{3, \mathbf{C}}+\Psi_{4}^{\mathrm{e}} I_{4, \mathbf{C}}+\Psi_{5}^{\mathrm{e}} I_{5, \mathbf{C}}\right) \\
& =2 \rho_{0}\left[\Psi_{1}^{\mathrm{e}} \mathbf{I}+\Psi_{2}^{\mathrm{e}}(\operatorname{tr} \mathbf{C I}-\mathbf{C})+\Psi_{3}^{\mathrm{e}} I_{3} \mathbf{C}^{-1}+\Psi_{4}^{\mathrm{e}} \mathbf{N}_{0}+\Psi_{5}^{\mathrm{e}} \Upsilon_{\mathbf{n}_{0} \mathbf{C}}\right] \\
& =2 \rho_{0}\left[\left(\Psi_{1}^{\mathrm{e}}+\Psi_{2}^{\mathrm{e}} I_{1}\right) \mathbf{I}-\Psi_{2}^{\mathrm{e}} \mathbf{C}+\Psi_{3}^{\mathrm{e}} I_{3} \mathbf{C}^{-1}+\Psi_{4}^{\mathrm{e}} \mathbf{N}_{0}+\Psi_{5}^{\mathrm{e}} \boldsymbol{\Upsilon}_{\mathbf{n}_{0} \mathbf{C}}\right]
\end{aligned}
$$

The viscous 2nd Piola-Kirchhoff stress is

$$
\begin{aligned}
\mathbf{S}^{\mathrm{v}}=2 \rho_{0} \frac{\partial \Psi^{\mathrm{v}}}{\partial \dot{\mathbf{C}}}=2 \rho_{0} \sum_{\beta=1}^{12}\left(\frac{\partial \Psi^{\mathrm{v}}}{\partial J_{\beta}} \frac{\partial J_{\beta}}{\partial \dot{\mathbf{C}}}\right)=2 \rho_{0} \sum_{\beta=1}^{12}\left(\Psi_{\beta}^{\mathrm{v}} J_{\beta, \dot{\mathbf{C}}}\right) \\
=2 \rho_{0}\left[\Psi_{1}^{\mathrm{v}} J_{1, \dot{\mathbf{C}}}+\Psi_{2}^{\mathrm{v}} J_{2, \dot{\mathbf{C}}}+\Psi_{3}^{\mathrm{v}} J_{3, \dot{\mathbf{C}}}+\Psi_{4}^{\mathrm{v}} J_{4, \dot{\mathbf{C}}}+\Psi_{5}^{\mathrm{v}} J_{5, \dot{\mathbf{C}}}+\Psi_{6}^{\mathrm{v}} J_{6, \dot{\mathbf{C}}}\right. \\
\left.\quad+\Psi_{7}^{\mathrm{v}} J_{7, \dot{\mathbf{C}}}+\Psi_{8}^{\mathrm{v}} J_{8, \dot{\mathbf{C}}}+\Psi_{9}^{\mathrm{v}} J_{9, \dot{\mathbf{C}}}+\Psi_{10}^{\mathrm{v}} J_{10, \dot{\mathbf{C}}}+\Psi_{11}^{\mathrm{v}} J_{11, \dot{\mathbf{C}}}+\Psi_{12}^{\mathrm{v}} J_{12, \dot{\mathbf{C}}}\right] \\
=2 \rho_{0}\left[\Psi_{1}^{\mathrm{v}} \mathbf{I}+\Psi_{2}^{\mathrm{v}} \dot{\mathbf{C}}+\Psi_{3}^{\mathrm{v}} J_{3} \dot{\mathbf{C}}^{-1}+\Psi_{4}^{\mathrm{v}} \mathbf{N}_{0}+\Psi_{5}^{\mathrm{v}} \boldsymbol{\Upsilon}_{\mathbf{n}_{0} \dot{\mathbf{C}}}+\Psi_{6}^{\mathrm{v}} \mathbf{C}+\Psi_{7}^{\mathrm{v}}(\mathbf{C} \dot{\mathbf{C}}+\dot{\mathbf{C}} \mathbf{C})\right. \\
\left.+\Psi_{8}^{\mathrm{v}} \mathbf{C}^{2}+\Psi_{9}^{\mathrm{v}}\left(\mathbf{C}^{2} \dot{\mathbf{C}}+\dot{\mathbf{C}} \mathbf{C}^{2}\right)+\Psi_{10}^{\mathrm{v}} \mathbf{C N}_{0}+\Psi_{11}^{\mathrm{v}}\left(\mathbf{C N}_{0} \dot{\mathbf{C}}+\dot{\mathbf{C}} \mathbf{C N} \mathbf{N}_{0}\right)+\Psi_{12}^{\mathrm{v}} \mathbf{C}^{2} \mathbf{N}_{0}\right] .
\end{aligned}
$$




\subsubsection{Elasticity tensor and viscosity tensor}

The response of the material to the applied strain and strain rate can be specified by the elasticity tensor and the viscosity tensor, respectively. The elasticity tensor $\mathbb{A}^{\mathbf{e}}$ and viscosity tensor $\mathbb{A}^{\mathbf{v}}$ are defined as

$$
\begin{gathered}
\mathbf{S}=\mathbf{S}^{\mathrm{e}}+\mathbf{S}^{\mathrm{v}} \stackrel{\text { def }}{=} \mathbb{A}^{\mathrm{e}}: \mathbf{C}+\mathbb{A}^{\mathrm{v}}: \dot{\mathbf{C}} \\
\text { where } \quad \mathbf{S}^{\mathrm{e}} \stackrel{\text { def }}{=} \mathbb{A}^{\mathrm{e}}: \mathbf{C} \text { and } \mathbf{S}^{\mathrm{v}} \stackrel{\text { def }}{=} \mathbb{A}^{\mathrm{v}}: \dot{\mathbf{C}} .
\end{gathered}
$$

By derivation of $\mathbf{S}^{\mathbf{e}}$ and $\mathbf{S}^{\mathbf{v}}$ with respect to $\mathbf{C}$ and $\dot{\mathbf{C}}$, respectively we obtain the material tensors:

$$
\frac{\partial \mathbf{S}^{\mathrm{e}}}{\partial \mathbf{C}}=\mathbb{A}_{, \mathbf{C}}^{\mathrm{e}}: \mathbf{C}+\mathbb{A}^{\mathrm{e}}: \mathbf{C}_{, \mathbf{C}}=\mathbb{A}_{, \mathbf{C}}^{\mathrm{e}}: \mathbf{C}+\mathbb{A}^{\mathrm{e}}: \mathbb{I}=\mathbb{A}_{, \mathbf{C}}^{\mathrm{e}}: \mathbf{C}+\mathbb{A}^{\mathrm{e}}
$$

where $\mathbb{A}^{\mathrm{e}}$ is the elasticity tensor and $\mathbb{A}_{, \mathbf{C}}^{\mathrm{e}}$ : $\mathbf{C}$ is the modified elasticity tensor due to mass change (growth) depending on the deformation in the case of an open system.

$$
\frac{\partial \mathbf{S}^{\mathrm{v}}}{\partial \dot{\mathbf{C}}}=\mathbb{A}_{, \dot{\mathbf{C}}}^{\mathrm{v}}: \dot{\mathbf{C}}+\mathbb{A}^{\mathrm{v}}: \dot{\mathbf{C}}_{, \dot{\mathrm{C}}}=\mathbb{A}_{, \dot{\mathbf{C}}}^{\mathrm{v}}: \dot{\mathbf{C}}+\mathbb{A}^{\mathrm{v}}: \mathbb{I}=\mathbb{A}_{, \dot{\mathbf{C}}}^{\mathrm{v}}: \dot{\mathbf{C}}+\mathbb{A}^{\mathrm{v}}
$$

where $\mathbb{A}^{\mathrm{v}}$ is the viscous tensor and $\mathbb{A}_{, \dot{\mathbf{C}}}^{\mathrm{v}}: \dot{\mathbf{C}}$ is the modified viscous tensor due to mass change (growth) depending on the deformation rate in the case of an open system.

On the other hand, the derivation of $\mathbf{S}^{\mathbf{e}}$ and $\mathbf{S}^{\mathbf{v}}$ in (4.3.10) with respect to $\mathbf{C}$ and $\dot{\mathbf{C}}$, respectively

$$
\begin{aligned}
\frac{\partial \mathbf{S}^{\mathbf{e}}}{\partial \mathbf{C}} & =2 \frac{\partial \rho_{0}}{\partial \mathbf{C}} \otimes \frac{\partial \Psi^{\mathrm{e}}}{\partial \mathbf{C}}+2 \rho_{0} \frac{\partial^{2} \Psi^{\mathrm{e}}}{\partial \mathbf{C}^{2}}, \\
\frac{\partial \mathbf{S}^{\mathrm{v}}}{\partial \dot{\mathbf{C}}} & =2 \frac{\partial \rho_{0}}{\partial \dot{\mathbf{C}}} \otimes \frac{\partial \Psi^{\mathrm{v}}}{\partial \dot{\mathbf{C}}}+2 \rho_{0} \frac{\partial^{2} \Psi^{\mathrm{v}}}{\partial \dot{\mathbf{C}}^{2}} .
\end{aligned}
$$

With comparing (4.3.38) and (4.3.40) we can deduce

$$
\mathbb{A}_{, \mathbf{C}}^{\mathrm{e}}: \mathbf{C} \stackrel{\text { def }}{=} \mathbb{A}^{\mathrm{meg}}=2 \frac{\partial \rho_{0}}{\partial \mathbf{C}} \otimes \frac{\partial \Psi^{\mathrm{e}}}{\partial \mathbf{C}} \quad \text { and } \quad \mathbb{A}^{\mathrm{e}}=2 \rho_{0} \frac{\partial^{2} \Psi^{\mathrm{e}}}{\partial \mathbf{C}^{2}}
$$

The fourth-order tensor $\mathbb{A}^{\text {meg }}$ is the modified elasticity tensor due to the mass change. 
With considering (3.2.5) and assuming that the mass source $\Pi^{\mathrm{S}}$ is activated by the elastic strain energy $\Psi^{\mathrm{e}}$, the term $2 \partial \rho_{0} / \partial \mathbf{C}$ can be written as

$$
2 \frac{\partial \rho_{0}}{\partial \mathbf{C}}=\frac{\partial \rho_{0}}{\partial \rho_{0}^{\mathrm{S}}} \cdot 2 \frac{\partial \rho_{0}^{\mathbf{S}}}{\partial \mathbf{C}}=\frac{\partial \rho_{0}}{\partial \rho_{0}^{\mathrm{S}}} \cdot 2 \int \frac{\partial \Pi^{\mathrm{S}}}{\partial \mathbf{C}} d t=\frac{\partial \rho_{0}}{\partial \rho_{0}^{\mathrm{S}}} \cdot 2 \int \frac{\partial \Pi^{\mathrm{S}}}{\partial \Psi^{\mathrm{e}}} \frac{\partial \Psi^{\mathrm{e}}}{\partial \mathbf{C}} d t
$$

Here, $\rho_{0}^{\mathrm{S}}$ is the solid mass density that can grow in a bioreactor.

If we introdue a scalar $\lambda^{\mathrm{Se}}$ defined as $\partial \Pi^{\mathrm{S}} / \partial \Psi^{\mathrm{e}}$ and consider (4.3.10), we obtain a second order tensor $\mathbf{A}^{\mathrm{e}}$

$$
\rightarrow \quad 2 \frac{\partial \rho_{0}^{\mathrm{S}}}{\partial \mathbf{C}}=2 \int \frac{\partial \Pi^{\mathrm{S}}}{\partial \Psi^{\mathrm{e}}} \frac{\partial \Psi^{\mathrm{e}}}{\partial \mathbf{C}} d t=\int \lambda^{\mathrm{Se}} \frac{\mathbf{S}^{\mathrm{e}}}{\rho_{0}} d t \stackrel{\text { def }}{=} \mathbf{A}^{\mathrm{e}}
$$

The physical meaning of $\lambda^{\text {Se }}$ is the change in mass source depending on strain energy (stimulation) change, in Yi et al. $[89,90]$. The second order tensor $\mathbf{A}^{\mathrm{e}}$ is the modified solid mass density depending on the deformation.

The modified elasticity tensor can be expressed by

$$
\mathbb{A}^{\mathrm{meg}}=\hat{\mathbb{A}}^{\mathrm{meg}}\left(\rho_{0}, \Psi^{\mathrm{e}}\right)=\frac{\partial \rho_{0}}{\partial \rho_{0}^{\mathrm{S}}} \mathbf{A}^{\mathrm{e}} \otimes \frac{\partial \Psi^{\mathrm{e}}}{\partial \mathbf{C}}
$$

In the present work, the mass source is assumed not to change in the bioreactor process (in other words, not activated due to deformation):

$$
\frac{\partial \Pi^{\mathrm{S}}}{\partial \Psi^{\mathrm{e}}}=0 \quad \rightarrow \quad \lambda^{\mathrm{Se}}=0 \quad \rightarrow \quad \mathbf{A}^{\mathrm{e}}=\mathbf{0} \quad \rightarrow \quad \mathrm{A}^{\mathrm{meg}}=\mathbb{0} .
$$

There is no modified elasticity tensor resulting from mass change.

In the same way, we can deduce the following equations by comparing (4.3.39) and (4.3.41):

$$
\mathbb{A}_{, \dot{\mathbf{C}}}^{\mathrm{v}}: \dot{\mathbf{C}} \stackrel{\text { def }}{=} \mathbb{A}^{\mathrm{mvg}}=2 \frac{\partial \rho_{0}}{\partial \dot{\mathbf{C}}} \otimes \frac{\partial \Psi^{\mathrm{v}}}{\partial \dot{\mathbf{C}}} \quad \text { and } \quad \mathbb{A}^{\mathrm{v}}=2 \rho_{0} \frac{\partial^{2} \Psi^{\mathrm{v}}}{\partial \dot{\mathbf{C}}^{2}}
$$

The fourth-order tensor $\mathbb{A}^{\mathrm{mvg}}$ is the modified viscosity tensor due to the mass change. 
By considering (3.2.5) and assuming that the mass source $\Pi^{\mathrm{S}}$ is activated by viscous potential, $\Psi^{\mathrm{v}}$, the term $2 \partial \rho_{0} / \partial \dot{\mathbf{C}}$ can be written as

$$
2 \frac{\partial \rho_{0}}{\partial \dot{\mathbf{C}}}=\frac{\partial \rho_{0}}{\partial \rho_{0}^{\mathbf{S}}} \cdot 2 \frac{\partial \rho_{0}^{\mathbf{S}}}{\partial \dot{\mathbf{C}}}=\frac{\partial \rho_{0}}{\partial \rho_{0}^{\mathbf{S}}} \cdot 2 \int \frac{\partial \Pi^{\mathrm{S}}}{\partial \dot{\mathbf{C}}} d t=\frac{\partial \rho_{0}}{\partial \rho_{0}^{\mathrm{S}}} \cdot 2 \int \frac{\partial \Pi^{\mathrm{S}}}{\partial \Psi^{\mathrm{v}}} \frac{\partial \Psi^{\mathrm{v}}}{\partial \dot{\mathbf{C}}} d t
$$

If we introdue a scalar $\lambda^{\mathrm{Sv}}$ defined as $\partial \Pi^{\mathrm{S}} / \partial \Psi^{\mathrm{v}}$ and consider (4.3.10), we obtain a second order tensor

$$
\rightarrow \quad 2 \frac{\partial \rho_{0}^{\mathrm{S}}}{\partial \dot{\mathbf{C}}}=2 \int \frac{\partial \Pi^{\mathrm{S}}}{\partial \Psi^{\mathrm{v}}} \frac{\partial \Psi^{\mathrm{v}}}{\partial \dot{\mathbf{C}}} d t=\int \lambda^{\mathrm{Sv}} \frac{\mathbf{S}^{\mathrm{v}}}{2 \rho_{0}} d t \stackrel{\text { def }}{=} \mathbf{A}^{\mathrm{v}}
$$

The physical meaning of $\lambda^{\mathrm{Sv}}$ is the change in mass source dependent on the viscous energy change. The second order tensor $\mathbf{A}^{\mathrm{v}}$ means a modified solid mass density dependent on the deformation rate.

The modified viscosity tensor can be expressed by

$$
\mathbb{A}^{\mathrm{mvg}}=\hat{\mathbb{A}}^{\mathrm{mvg}}\left(\rho_{0}, \Psi^{\mathrm{v}}\right)=\frac{\partial \rho_{0}}{\partial \rho_{0}^{\mathrm{s}}} \mathbf{A}^{\mathrm{v}} \otimes \frac{\partial \Psi^{\mathrm{v}}}{\partial \dot{\mathbf{C}}}
$$

As the solid mass is assumed not to be activated dependent on deformation rate in a bioreactor process, then

$$
\frac{\partial \Pi^{\mathrm{S}}}{\partial \Psi^{\mathrm{v}}}=0 \quad \rightarrow \quad \lambda^{\mathrm{Sv}}=0 \quad \rightarrow \quad \mathbf{A}^{\mathrm{v}}=\mathbf{0} \quad \rightarrow \quad \mathbb{A}^{\mathrm{mvg}}=\mathbb{0}
$$

There is no modification of the viscosity tensor in consequence of the mass change in the bioreactor process.

For the sake of convenience, we define the following symbols for the derivations of the free energies with respect to its invariants:

$$
\Psi_{\alpha \beta}^{\mathrm{e}}=\frac{\partial^{2} \Psi^{\mathrm{e}}}{\partial I_{\alpha} \partial I_{\beta}}, \quad \Psi_{\alpha \beta}^{\mathrm{v}}=\frac{\partial^{2} \Psi^{\mathrm{v}}}{\partial J_{\alpha} \partial J_{\beta}} .
$$

In our case of constant mass, a closed system, we have $\mathbb{A}^{\text {meg }}=\mathbb{A}^{\text {mvg }}=\mathbb{O}$ and the elasticity tensor $\mathbb{A}^{\mathrm{e}}$ and viscosity tensor $\mathbb{A}^{\mathrm{v}}$ can be calculated as follows. 
Elasticity tensor $\mathbb{A}^{e}$ :

$$
\begin{aligned}
\mathbb{A}^{\mathrm{e}} & =2 \rho_{0} \frac{\partial^{2} \Psi^{\mathrm{e}}}{\partial \mathbf{C}^{2}}=\frac{\partial \mathbf{S}^{\mathrm{e}}}{\partial \mathbf{C}}=2 \rho_{0} \frac{\partial}{\partial \mathbf{C}} \sum_{\alpha=1}^{5}\left(\Psi_{\alpha}^{\mathrm{e}} I_{\alpha, \mathbf{C}}\right) \\
& =2 \rho_{0} \frac{\partial}{\partial \mathbf{C}}\left[\left(\Psi_{1}^{\mathrm{e}}+\Psi_{2}^{\mathrm{e}} I_{1}\right) \mathbf{I}-\Psi_{2}^{\mathrm{e}} \mathbf{C}+\Psi_{3}^{\mathrm{e}} I_{3} \mathbf{C}^{-1}+\Psi_{4}^{\mathrm{e}} \mathbf{N}_{0}+\Psi_{5}^{\mathrm{e}} \boldsymbol{\Upsilon}_{\mathbf{n}_{0} \mathbf{C}}\right] \\
\frac{\mathbb{A}^{\mathrm{e}}}{2 \rho_{0}}= & \mathbf{I} \odot\left(\Psi_{1}^{\mathrm{e}}+\Psi_{2}^{\mathrm{e}} I_{1}\right)_{, \mathbf{C}}+\left(\Psi_{1}^{\mathrm{e}}+\Psi_{2}^{\mathrm{e}} I_{1}\right) \mathbf{I}_{, \mathbf{C}}-\mathbf{C} \odot \Psi_{2, \mathbf{C}}^{\mathrm{e}}-\Psi_{2}^{\mathrm{e}} \mathbf{C}_{, \mathbf{C}} \\
+ & \mathbf{C}^{-1} \odot\left(\Psi_{3}^{\mathrm{e}} I_{3}\right)_{, \mathbf{C}}+\Psi_{3}^{\mathrm{e}} I_{3} \mathbf{C}_{, \mathbf{C}}^{-1}+\mathbf{N}_{0} \odot \Psi_{4, \mathbf{C}}^{\mathrm{e}}+\Psi_{4}^{\mathrm{e}} \mathbf{N}_{0, \mathbf{C}}+\boldsymbol{\Upsilon}_{\mathbf{n}_{0} \mathbf{C}} \odot \Psi_{5, \mathbf{C}}^{\mathrm{e}}+\Psi_{5}^{\mathrm{e}} \boldsymbol{\Upsilon}_{\mathbf{n}_{0} \mathbf{C}, \mathbf{C}}
\end{aligned}
$$

With the identities (A.3.10), (A.3.11), and (A.3.12), we obtain

$$
\begin{aligned}
& \mathbf{N}_{0, \mathbf{C}}=\mathbb{O} \\
& \Upsilon_{\mathbf{n}_{0} \mathbf{C}} \stackrel{\text { def }}{=} \mathbf{N}_{0} \mathbf{C}+\mathbf{C N}_{0} \\
& \Upsilon_{\mathbf{n}_{0} \mathbf{C}, \mathbf{C}}=\left(\mathbf{N}_{0} \mathbf{C}+\mathbf{C N}_{0}\right)_{, \mathbf{C}}=\mathbf{N}_{0} \mathbf{C}_{, \mathbf{C}}+\mathbf{C}_{\mathbf{C}_{\mathbf{C}}} \mathbf{N}_{0}=\mathbf{N}_{0} \mathbf{I}+\mathbb{I N}_{0}=\mathbf{N}_{0} \otimes \mathbf{I}+\mathbf{I} \otimes \mathbf{N}_{0} \text { and } \\
& \Psi_{\omega, \mathbf{C}}^{\mathrm{e}}=\sum_{\alpha=1}^{5}\left(\Psi_{\omega \alpha}^{\mathrm{e}} I_{\alpha, \mathbf{C}}\right), \quad \text { where } \omega=1, \cdots 5 \text {. } \\
& \frac{\mathbb{A}^{\mathrm{e}}}{2 \rho_{0}}=\mathbf{I} \odot\left(\Psi_{1}^{\mathrm{e}}+\Psi_{2}^{\mathrm{e}} I_{1}\right)_{, \mathbf{C}}-\mathbf{C} \odot \Psi_{2, \mathbf{C}}^{\mathrm{e}}-\Psi_{2}^{\mathrm{e}} \mathrm{I} \\
& +\mathbf{C}^{-1} \odot\left(\Psi_{3}^{\mathrm{e}} I_{3}\right)_{, \mathbf{C}}+\Psi_{3}^{\mathrm{e}} I_{3}\left(-\mathbf{C}^{-1} \otimes \mathbf{C}^{-1}\right)+\mathbf{N}_{0} \odot \Psi_{4, \mathbf{C}}^{\mathrm{e}} \\
& +\Upsilon_{\mathbf{n}_{0} \mathbf{C}} \odot \Psi_{5, \mathbf{C}}^{\mathrm{e}}+\Psi_{5}^{\mathrm{e}}\left(\mathbf{N}_{0} \otimes \mathbf{I}+\mathbf{I} \otimes \mathbf{N}_{0}\right) \\
& =\mathbf{I} \odot\left[\sum_{\alpha=1}^{5} \Psi_{1 \alpha}^{\mathrm{e}} I_{\alpha, \mathbf{C}}+\left(\sum_{\alpha=1}^{5} \Psi_{2 \alpha}^{\mathrm{e}} I_{\alpha, \mathbf{C}}\right) I_{1}+\Psi_{2}^{\mathrm{e}} I_{1, \mathbf{C}}\right]-\mathbf{C} \odot \sum_{\alpha=1}^{5} \Psi_{2 \alpha}^{\mathrm{e}} I_{\alpha, \mathbf{C}}-\Psi_{2}^{\mathrm{e}} \mathrm{I} \\
& +\mathbf{C}^{-1} \odot\left[\left(\sum_{\alpha=1}^{5} \Psi_{3 \alpha}^{\mathrm{e}} I_{\alpha, \mathbf{C}}\right) I_{3}+\Psi_{3}^{\mathrm{e}} I_{3, \mathbf{C}}\right]-\Psi_{3}^{\mathrm{e}} I_{3} \mathbf{C}^{-1} \otimes \mathbf{C}^{-1} \\
& +\mathbf{N}_{0} \odot \sum_{\alpha=1}^{5} \Psi_{4 \alpha}^{\mathrm{e}} I_{\alpha, \mathbf{C}} \\
& +\Upsilon_{\mathbf{n}_{0} \mathbf{C}} \odot \sum_{\alpha=1}^{5} \Psi_{5 \alpha}^{\mathrm{e}} I_{\alpha, \mathbf{C}}+\Psi_{5}^{\mathrm{e}}\left(\mathbf{N}_{0} \otimes \mathbf{I}+\mathbf{I} \otimes \mathbf{N}_{0}\right)
\end{aligned}
$$




$$
\begin{aligned}
& \rightarrow \quad \frac{\mathbb{A}^{\mathrm{e}}}{2 \rho_{0}}=\mathbf{I} \odot\left[\Psi_{11}^{\mathrm{e}} I_{1, \mathbf{C}}+\Psi_{12}^{\mathrm{e}} I_{2, \mathbf{C}}+\Psi_{13}^{\mathrm{e}} I_{3, \mathbf{C}}+\Psi_{14}^{\mathrm{e}} I_{4, \mathbf{C}}+\Psi_{15}^{\mathrm{e}} I_{5, \mathbf{C}}\right. \\
& \left.+\left(\Psi_{21}^{\mathrm{e}} I_{1, \mathbf{C}}+\Psi_{22}^{\mathrm{e}} I_{2, \mathbf{C}}+\Psi_{23}^{\mathrm{e}} I_{3, \mathbf{C}}+\Psi_{24}^{\mathrm{e}} I_{4, \mathbf{C}}+\Psi_{25}^{\mathrm{e}} I_{5, \mathbf{C}}\right) I_{1}+\Psi_{2}^{\mathrm{e}} I_{1, \mathbf{C}}\right] \\
& -\mathbf{C} \odot\left(\Psi_{21}^{\mathrm{e}} I_{1, \mathbf{C}}+\Psi_{22}^{\mathrm{e}} I_{2, \mathbf{C}}+\Psi_{23}^{\mathrm{e}} I_{3, \mathbf{C}}+\Psi_{24}^{\mathrm{e}} I_{4, \mathbf{C}}+\Psi_{25}^{\mathrm{e}} I_{5, \mathbf{C}}\right)-\Psi_{2}^{\mathrm{e}} \mathrm{I} \\
& +\mathbf{C}^{-1} \odot\left[\left(\Psi_{31}^{\mathrm{e}} I_{1, \mathbf{C}}+\Psi_{32}^{\mathrm{e}} I_{2, \mathbf{C}}+\Psi_{33}^{\mathrm{e}} I_{3, \mathbf{C}}+\Psi_{34}^{\mathrm{e}} I_{4, \mathbf{C}}+\Psi_{35}^{\mathrm{e}} I_{5, \mathbf{C}}\right) I_{3}+\Psi_{3}^{\mathrm{e}} I_{3, \mathbf{C}}\right] \\
& -\Psi_{3}^{\mathrm{e}} I_{3} \mathbf{C}^{-1} \otimes \mathbf{C}^{-1}+\mathbf{N}_{0} \odot\left(\Psi_{41}^{\mathrm{e}} I_{1, \mathbf{C}}+\Psi_{42}^{\mathrm{e}} I_{2, \mathbf{C}}+\Psi_{43}^{\mathrm{e}} I_{3, \mathbf{C}}+\Psi_{44}^{\mathrm{e}} I_{4, \mathbf{C}}+\Psi_{45}^{\mathrm{e}} I_{5, \mathbf{C}}\right) \\
& +\Upsilon_{\mathbf{n}_{0} \mathbf{C}} \odot\left(\Psi_{51}^{\mathrm{e}} I_{1, \mathbf{C}}+\Psi_{52}^{\mathrm{e}} I_{2, \mathbf{C}}+\Psi_{53}^{\mathrm{e}} I_{3, \mathbf{C}}+\Psi_{54}^{\mathrm{e}} I_{4, \mathbf{C}}+\Psi_{55}^{\mathrm{e}} I_{5, \mathbf{C}}\right)+\Psi_{5}^{\mathrm{e}}\left(\mathbf{N}_{0} \otimes \mathbf{I}+\mathbf{I} \otimes \mathbf{N}_{0}\right) \\
& =\mathbf{I} \odot\left[\left(\Psi_{11}^{\mathrm{e}}+\Psi_{21}^{\mathrm{e}} I_{1}+\Psi_{2}^{\mathrm{e}}\right) I_{1, \mathbf{C}}+\left(\Psi_{12}^{\mathrm{e}}+\Psi_{22}^{\mathrm{e}} I_{1}\right) I_{2, \mathbf{C}}\right. \\
& \left.+\left(\Psi_{13}^{\mathrm{e}}+\Psi_{23}^{\mathrm{e}} I_{1}\right) I_{3, \mathbf{C}}+\left(\Psi_{14}^{\mathrm{e}}+\Psi_{24}^{\mathrm{e}} I_{1}\right) I_{4, \mathbf{C}}+\left(\Psi_{15}^{\mathrm{e}}+\Psi_{25}^{\mathrm{e}} I_{1}\right) I_{5, \mathbf{C}}\right] \\
& -\mathbf{C} \odot\left(\Psi_{21}^{\mathrm{e}} I_{1, \mathbf{C}}+\Psi_{22}^{\mathrm{e}} I_{2, \mathbf{C}}+\Psi_{23}^{\mathrm{e}} I_{3, \mathbf{C}}+\Psi_{24}^{\mathrm{e}} I_{4, \mathbf{C}}+\Psi_{25}^{\mathrm{e}} I_{5, \mathbf{C}}\right) \\
& +\mathbf{C}^{-1} \odot I_{3}\left[\Psi_{31}^{\mathrm{e}} I_{1, \mathbf{C}}+\Psi_{32}^{\mathrm{e}} I_{2, \mathbf{C}}+\left(\Psi_{33}^{\mathrm{e}}+\frac{\Psi_{3}^{\mathrm{e}}}{I_{3}}\right) I_{3, \mathbf{C}}+\Psi_{34}^{\mathrm{e}} I_{4, \mathbf{C}}+\Psi_{35}^{\mathrm{e}} I_{5, \mathbf{C}}\right] \\
& +\mathbf{N}_{0} \odot\left(\Psi_{41}^{\mathrm{e}} I_{1, \mathbf{C}}+\Psi_{42}^{\mathrm{e}} I_{2, \mathbf{C}}+\Psi_{43}^{\mathrm{e}} I_{3, \mathbf{C}}+\Psi_{44}^{\mathrm{e}} I_{4, \mathbf{C}}+\Psi_{45}^{\mathrm{e}} I_{5, \mathbf{C}}\right) \\
& +\Upsilon_{\mathbf{n}_{0} \mathbf{C}} \odot\left(\Psi_{51}^{\mathrm{e}} I_{1, \mathbf{C}}+\Psi_{52}^{\mathrm{e}} I_{2, \mathbf{C}}+\Psi_{53}^{\mathrm{e}} I_{3, \mathbf{C}}+\Psi_{54}^{\mathrm{e}} I_{4, \mathbf{C}}+\Psi_{55}^{\mathrm{e}} I_{5, \mathbf{C}}\right) \\
& -\Psi_{2}^{\mathrm{e}} \mathrm{I}-\Psi_{3}^{\mathrm{e}} I_{3} \mathbf{C}^{-1} \otimes \mathbf{C}^{-1}+\Psi_{5}^{\mathrm{e}}\left(\mathbf{N}_{0} \otimes \mathbf{I}+\mathbf{I} \otimes \mathbf{N}_{0}\right) \\
& =\mathbf{I} \odot\left[\left(\Psi_{11}^{\mathrm{e}}+\Psi_{21}^{\mathrm{e}} I_{1}+\Psi_{2}^{\mathrm{e}}\right) \mathbf{I}+\left(\Psi_{12}^{\mathrm{e}}+\Psi_{22}^{\mathrm{e}} I_{1}\right)(\operatorname{tr} \mathbf{C I}-\mathbf{C})\right. \\
& \left.+\left(\Psi_{13}^{\mathrm{e}}+\Psi_{23}^{\mathrm{e}} I_{1}\right) I_{3} \mathbf{C}^{-1}+\left(\Psi_{14}^{\mathrm{e}}+\Psi_{24}^{\mathrm{e}} I_{1}\right) \mathbf{N}_{0}+\left(\Psi_{15}^{\mathrm{e}}+\Psi_{25}^{\mathrm{e}} I_{1}\right) \Upsilon_{\mathbf{n}_{0} \mathbf{C}}\right] \\
& -\mathbf{C} \odot\left[\Psi_{21}^{\mathrm{e}} \mathbf{I}+\Psi_{22}^{\mathrm{e}}(\operatorname{tr} \mathbf{C I}-\mathbf{C})+\Psi_{23}^{\mathrm{e}} I_{3} \mathbf{C}^{-1}+\Psi_{24}^{\mathrm{e}} \mathbf{N}_{0}+\Psi_{25}^{\mathrm{e}} \Upsilon_{\mathbf{n}_{0} \mathbf{C}}\right] \\
& +\mathbf{C}^{-1} \odot I_{3}\left[\Psi_{31}^{\mathrm{e}} \mathbf{I}+\Psi_{32}^{\mathrm{e}}(\operatorname{tr} \mathbf{C I}-\mathbf{C})+\left(\Psi_{33}^{\mathrm{e}}+\frac{\Psi_{3}^{\mathrm{e}}}{I_{3}}\right) I_{3} \mathbf{C}^{-1}+\Psi_{34}^{\mathrm{e}} \mathbf{N}_{0}+\Psi_{35}^{\mathrm{e}} \boldsymbol{\Upsilon}_{\mathbf{n}_{0} \mathbf{C}}\right] \\
& +\mathbf{N}_{0} \odot\left[\Psi_{41}^{\mathrm{e}} \mathbf{I}+\Psi_{42}^{\mathrm{e}}(\operatorname{tr} \mathbf{C I}-\mathbf{C})+\Psi_{43}^{\mathrm{e}} I_{3} \mathbf{C}^{-1}+\Psi_{44}^{\mathrm{e}} \mathbf{N}_{0}+\Psi_{45}^{\mathrm{e}} \boldsymbol{\Upsilon}_{\mathbf{n}_{0} \mathbf{C}}\right] \\
& +\Upsilon_{\mathbf{n}_{0} \mathbf{C}} \odot\left[\Psi_{51}^{\mathrm{e}} \mathbf{I}+\Psi_{52}^{\mathrm{e}}(\operatorname{tr} \mathbf{C I}-\mathbf{C})+\Psi_{53}^{\mathrm{e}} I_{3} \mathbf{C}^{-1}+\Psi_{54}^{\mathrm{e}} \mathbf{N}_{0}+\Psi_{55}^{\mathrm{e}} \Upsilon_{\mathbf{n}_{0} \mathbf{C}}\right] \\
& -\Psi_{2}^{\mathrm{e}} \mathrm{I}-\Psi_{3}^{\mathrm{e}} I_{3} \mathbf{C}^{-1} \otimes \mathbf{C}^{-1}+\Psi_{5}^{\mathrm{e}}\left(\mathbf{N}_{0} \otimes \mathbf{I}+\mathbf{I} \otimes \mathbf{N}_{0}\right)
\end{aligned}
$$




$$
\begin{aligned}
& \rightarrow \frac{\mathbb{A}^{\mathrm{e}}}{2 \rho_{0}}=\mathbf{I} \odot\left[\left(\Psi_{11}^{\mathrm{e}}+\Psi_{21}^{\mathrm{e}} I_{1}+\Psi_{2}^{\mathrm{e}}+\left(\Psi_{12}^{\mathrm{e}}+\Psi_{22}^{\mathrm{e}} I_{1}\right) \operatorname{tr} \mathbf{C}\right) \mathbf{I}-\left(\Psi_{12}^{\mathrm{e}}+\Psi_{22}^{\mathrm{e}} I_{1}\right) \mathbf{C}\right. \\
& \left.+\left(\Psi_{13}^{\mathrm{e}}+\Psi_{23}^{\mathrm{e}} I_{1}\right) I_{3} \mathbf{C}^{-1}+\left(\Psi_{14}^{\mathrm{e}}+\Psi_{24}^{\mathrm{e}} I_{1}\right) \mathbf{N}_{0}+\left(\Psi_{15}^{\mathrm{e}}+\Psi_{25}^{\mathrm{e}} I_{1}\right) \Upsilon_{\mathbf{n}_{0} \mathbf{C}}\right] \\
& -\mathbf{C} \odot\left[\left(\Psi_{21}^{\mathrm{e}}+\Psi_{22}^{\mathrm{e}} \operatorname{tr} \mathbf{C}\right) \mathbf{I}-\Psi_{22}^{\mathrm{e}} \mathbf{C}+\Psi_{23}^{\mathrm{e}} I_{3} \mathbf{C}^{-1}+\Psi_{24}^{\mathrm{e}} \mathbf{N}_{0}+\Psi_{25}^{\mathrm{e}} \Upsilon_{\mathbf{n}_{0} \mathbf{C}}\right] \\
& +\mathbf{C}^{-1} \odot I_{3}\left[\left(\Psi_{31}^{\mathrm{e}}+\Psi_{32}^{\mathrm{e}} \operatorname{tr} \mathbf{C}\right) \mathbf{I}-\Psi_{32}^{\mathrm{e}} \mathbf{C}+\left(\Psi_{33}^{\mathrm{e}} I_{3}+\Psi_{3}^{\mathrm{e}}\right) \mathbf{C}^{-1}+\Psi_{34}^{\mathrm{e}} \mathbf{N}_{0}+\Psi_{35}^{\mathrm{e}} \boldsymbol{\Upsilon}_{\mathbf{n}_{0} \mathbf{C}}\right] \\
& +\mathbf{N}_{0} \odot\left[\left(\Psi_{41}^{\mathrm{e}}+\Psi_{42}^{\mathrm{e}} \operatorname{tr} \mathbf{C}\right) \mathbf{I}-\Psi_{42}^{\mathrm{e}} \mathbf{C}+\Psi_{43}^{\mathrm{e}} I_{3} \mathbf{C}^{-1}+\Psi_{44}^{\mathrm{e}} \mathbf{N}_{0}+\Psi_{45}^{\mathrm{e}} \Upsilon_{\mathbf{n}_{0} \mathbf{C}}\right] \\
& +\Upsilon_{\mathbf{n}_{0} \mathbf{C}} \odot\left[\left(\Psi_{51}^{\mathrm{e}}+\Psi_{52}^{\mathrm{e}} \operatorname{tr} \mathbf{C}\right) \mathbf{I}-\Psi_{52}^{\mathrm{e}} \mathbf{C}+\Psi_{53}^{\mathrm{e}} I_{3} \mathbf{C}^{-1}+\Psi_{54}^{\mathrm{e}} \mathbf{N}_{0}+\Psi_{55}^{\mathrm{e}} \Upsilon_{\mathbf{n}_{0} \mathbf{C}}\right] \\
& -\Psi_{2}^{\mathrm{e}} \mathrm{I}-\Psi_{3}^{\mathrm{e}} I_{3} \mathbf{C}^{-1} \otimes \mathbf{C}^{-1}+\Psi_{5}^{\mathrm{e}}\left(\mathbf{N}_{0} \otimes \mathbf{I}+\mathbf{I} \otimes \mathbf{N}_{0}\right)
\end{aligned}
$$

With the identities $\operatorname{tr} \mathbf{C}=I_{1}, \Psi_{\alpha \beta}^{\mathrm{e}}=\Psi_{\beta \alpha}^{\mathrm{e}}$, it can be simplified

$$
\begin{aligned}
& \frac{\mathbb{A}^{\mathrm{e}}}{2 \rho_{0}}=\mathbf{I} \odot\left[\left(\Psi_{11}^{\mathrm{e}}+2 \Psi_{12}^{\mathrm{e}} I_{1}+\Psi_{2}^{\mathrm{e}}+\Psi_{22}^{\mathrm{e}} I_{1}^{2}\right) \mathbf{I}-\left(\Psi_{12}^{\mathrm{e}}+\Psi_{22}^{\mathrm{e}} I_{1}\right) \mathbf{C}\right. \\
& \left.+\left(\Psi_{13}^{\mathrm{e}}+\Psi_{23}^{\mathrm{e}} I_{1}\right) I_{3} \mathbf{C}^{-1}+\left(\Psi_{14}^{\mathrm{e}}+\Psi_{24}^{\mathrm{e}} I_{1}\right) \mathbf{N}_{0}+\left(\Psi_{15}^{\mathrm{e}}+\Psi_{25}^{\mathrm{e}} I_{1}\right) \Upsilon_{\mathbf{n}_{0} \mathbf{C}}\right] \\
& -\mathbf{C} \odot\left[\left(\Psi_{12}^{\mathrm{e}}+\Psi_{22}^{\mathrm{e}} I_{1}\right) \mathbf{I}-\Psi_{22}^{\mathrm{e}} \mathbf{C}+\Psi_{23}^{\mathrm{e}} I_{3} \mathbf{C}^{-1}+\Psi_{24}^{\mathrm{e}} \mathbf{N}_{0}+\Psi_{25}^{\mathrm{e}} \mathbf{\Upsilon}_{\mathbf{n}_{0}} \mathbf{C}\right] \\
& +\mathbf{C}^{-1} \odot I_{3}\left[\left(\Psi_{13}^{\mathrm{e}}+\Psi_{23}^{\mathrm{e}} I_{1}\right) \mathbf{I}-\Psi_{23}^{\mathrm{e}} \mathbf{C}+\left(\Psi_{33}^{\mathrm{e}} I_{3}+\Psi_{3}^{\mathrm{e}}\right) \mathbf{C}^{-1}+\Psi_{34}^{\mathrm{e}} \mathbf{N}_{0}+\Psi_{35}^{\mathrm{e}} \Upsilon_{\mathbf{n}_{0} \mathbf{C}}\right] \\
& +\mathbf{N}_{0} \odot\left[\left(\Psi_{14}^{\mathrm{e}}+\Psi_{24}^{\mathrm{e}} I_{1}\right) \mathbf{I}-\Psi_{24}^{\mathrm{e}} \mathbf{C}+\Psi_{34}^{\mathrm{e}} I_{3} \mathbf{C}^{-1}+\Psi_{44}^{\mathrm{e}} \mathbf{N}_{0}+\Psi_{45}^{\mathrm{e}} \Upsilon_{\mathbf{n}_{0} \mathbf{C}}\right] \\
& +\Upsilon_{\mathbf{n}_{0} \mathbf{C}} \odot\left[\left(\Psi_{15}^{\mathrm{e}}+\Psi_{25}^{\mathrm{e}} I_{1}\right) \mathbf{I}-\Psi_{25}^{\mathrm{e}} \mathbf{C}+\Psi_{35}^{\mathrm{e}} I_{3} \mathbf{C}^{-1}+\Psi_{45}^{\mathrm{e}} \mathbf{N}_{0}+\Psi_{55}^{\mathrm{e}} \Upsilon_{\mathbf{n}_{0} \mathbf{C}}\right] \\
& -\Psi_{2}^{\mathrm{e}} \mathrm{I}-\Psi_{3}^{\mathrm{e}} I_{3} \mathbf{C}^{-1} \otimes \mathbf{C}^{-1}+\Psi_{5}^{\mathrm{e}}\left(\mathbf{N}_{0} \otimes \mathbf{I}+\mathbf{I} \otimes \mathbf{N}_{0}\right)
\end{aligned}
$$


Viscosity tensor $\mathbb{A}^{\mathrm{v}}$ :

$$
\begin{aligned}
& \mathbb{A}^{\mathrm{v}}=2 \rho_{0} \frac{\partial^{2} \Psi^{\mathrm{v}}}{\partial \dot{\mathbf{C}}^{2}}=\frac{\partial \mathbf{S}^{\mathrm{v}}}{\partial \dot{\mathbf{C}}}=2 \rho_{0} \frac{\partial}{\partial \dot{\mathbf{C}}} \sum_{\beta=1}^{12}\left(\Psi_{\beta}^{\mathrm{v}} J_{\beta, \dot{\mathbf{C}}}\right) \\
& =2 \rho_{0} \frac{\partial}{\partial \dot{\mathbf{C}}}\left[\Psi_{1}^{\mathrm{v}} \mathbf{I}+\Psi_{2}^{\mathrm{v}} \dot{\mathbf{C}}+\Psi_{3}^{\mathrm{v}} J_{3} \dot{\mathbf{C}}^{-1}+\Psi_{4}^{\mathrm{v}} \mathbf{N}_{0}+\Psi_{5}^{\mathrm{v}} \boldsymbol{\Upsilon}_{\mathbf{n}_{0} \dot{\mathbf{C}}}+\Psi_{6}^{\mathrm{v}} \mathbf{C}+\Psi_{7}^{\mathrm{v}}(\mathbf{C} \dot{\mathbf{C}}+\dot{\mathbf{C}} \mathbf{C})\right. \\
& \left.+\Psi_{8}^{\mathrm{v}} \mathbf{C}^{2}+\Psi_{9}^{\mathrm{v}}\left(\mathbf{C}^{2} \dot{\mathbf{C}}+\dot{\mathbf{C}} \mathbf{C}^{2}\right)+\Psi_{10}^{\mathrm{v}} \mathbf{C N}_{0}+\Psi_{11}^{\mathrm{v}}\left(\mathbf{C N}_{0} \dot{\mathbf{C}}+\dot{\mathbf{C}} \mathbf{C N} \mathbf{N}_{0}\right)+\Psi_{12}^{\mathrm{v}} \mathbf{C}^{2} \mathbf{N}_{0}\right] \\
& \frac{\mathbb{A}^{\mathrm{v}}}{2 \rho_{0}}=\frac{\partial}{\partial \dot{\mathbf{C}}}\left[\Psi_{1}^{\mathrm{v}} \mathbf{I}+\Psi_{2}^{\mathrm{v}} \dot{\mathbf{C}}+\Psi_{3}^{\mathrm{v}} J_{3} \dot{\mathbf{C}}^{-1}+\Psi_{4}^{\mathrm{v}} \mathbf{N}_{0}+\Psi_{5}^{\mathrm{v}} \boldsymbol{\Upsilon}_{\mathbf{n}_{0} \dot{\mathbf{C}}}+\Psi_{6}^{\mathrm{v}} \mathbf{C}+\Psi_{7}^{\mathrm{v}}(\mathbf{C} \dot{\mathbf{C}}+\dot{\mathbf{C}} \mathbf{C})\right. \\
& \left.+\Psi_{8}^{\mathrm{v}} \mathbf{C}^{2}+\Psi_{9}^{\mathrm{v}}\left(\mathbf{C}^{2} \dot{\mathbf{C}}+\dot{\mathbf{C}} \mathbf{C}^{2}\right)+\Psi_{10}^{\mathrm{v}} \mathbf{C N}_{0}+\Psi_{11}^{\mathrm{v}}\left(\mathbf{C N}_{0} \dot{\mathbf{C}}+\dot{\mathbf{C}} \mathbf{C N}_{0}\right)+\Psi_{12}^{\mathrm{v}} \mathbf{C}^{2} \mathbf{N}_{0}\right] \\
& =\mathbf{I} \odot \Psi_{1, \dot{\mathbf{C}}}^{\mathrm{v}}+\Psi_{1, \dot{\mathbf{C}}}^{\mathrm{v}}+\dot{\mathbf{C}} \odot \Psi_{2, \dot{\mathbf{C}}}^{\mathrm{v}}+\Psi_{2}^{\mathrm{v}} \dot{\mathbf{C}}_{, \dot{\mathbf{C}}}+\dot{\mathbf{C}}^{-1} \odot\left(\Psi_{3}^{\mathrm{v}} J_{3}\right)_{, \dot{\mathbf{C}}}+\Psi_{3}^{\mathrm{v}} J_{3} \dot{\mathbf{C}}_{, \dot{\mathbf{C}}}^{-1} \\
& +\mathbf{N}_{0} \odot \Psi_{4, \dot{\mathbf{C}}}^{\mathrm{v}}+\Psi_{4}^{\mathrm{v}} \mathbf{N}_{0, \dot{\mathbf{C}}}+\Upsilon_{\mathbf{n}_{0} \dot{\mathbf{C}}} \odot \Psi_{5, \dot{\mathbf{C}}}^{\mathrm{v}}+\Psi_{5}^{\mathrm{v}} \Upsilon_{\mathbf{n}_{0} \dot{\mathbf{C}}, \dot{\mathbf{C}}}+\mathbf{C} \odot \Psi_{6, \dot{\mathbf{C}}}^{\mathrm{v}}+\Psi_{6}^{\mathrm{v}} \mathbf{C}_{, \dot{\mathbf{C}}} \\
& +(\mathbf{C} \dot{\mathbf{C}}+\dot{\mathbf{C C}}) \odot \Psi_{7, \mathbf{C}}^{\mathrm{v}}+\Psi_{7}^{\mathrm{v}}(\mathbf{C} \dot{\mathbf{C}}+\dot{\mathbf{C C}})_{, \dot{\mathbf{C}}}+\mathbf{C}^{2} \odot \Psi_{8, \dot{\mathbf{C}}}^{\mathrm{v}}+\Psi_{8}^{\mathrm{v}} \mathbf{C}_{, \dot{\mathbf{C}}}^{2} \\
& +\left(\mathbf{C}^{2} \dot{\mathbf{C}}+\dot{\mathbf{C}} \mathbf{C}^{2}\right) \odot \Psi_{9, \dot{\mathbf{C}}}^{\mathrm{v}}+\Psi_{9}^{\mathrm{v}}\left(\mathbf{C}^{2} \dot{\mathbf{C}}+\dot{\mathbf{C}} \mathbf{C}^{2}\right)_{, \dot{\mathbf{C}}}+\mathbf{C N}_{0} \odot \Psi_{10, \dot{\mathbf{C}}}^{\mathrm{v}}+\Psi_{10}^{\mathrm{v}}\left(\mathbf{C N}_{0}\right)_{, \dot{\mathbf{C}}} \\
& +\left(\mathbf{C N}_{0} \dot{\mathbf{C}}+\dot{\mathbf{C}} \mathbf{C N}_{0}\right) \odot \Psi_{11, \dot{\mathbf{C}}}^{\mathrm{v}}+\Psi_{11}^{\mathrm{v}}\left(\mathbf{C N}_{0} \dot{\mathbf{C}}+\dot{\mathbf{C}} \mathbf{C N}_{0}\right)_{, \dot{\mathbf{C}}} \\
& +\mathbf{C}^{2} \mathbf{N}_{0} \odot \Psi_{12, \dot{\mathbf{C}}}^{\mathrm{v}}+\Psi_{12}^{\mathrm{v}}\left(\mathbf{C}^{2} \mathbf{N}_{0}\right)_{, \dot{\mathbf{C}}}
\end{aligned}
$$


With the identities (A.3.10), (A.3.11) (A.3.12) we obtain

$$
\begin{aligned}
& \mathbf{N}_{0, \dot{\mathbf{C}}}=\mathbb{0}, \\
& \mathbf{\Upsilon}_{\mathbf{n}_{0} \dot{\mathbf{C}}, \dot{\mathbf{C}}}=\left(\mathbf{N}_{0} \dot{\mathbf{C}}+\dot{\mathbf{C}}_{0}\right)_{, \dot{\mathbf{C}}}=\mathbf{N}_{0} \dot{\mathbf{C}}_{, \dot{\mathbf{C}}}+\dot{\mathbf{C}}_{, \dot{\mathbf{C}}} \mathbf{N}_{0}=\mathbf{N}_{0} \mathbf{I}+\mathbb{I N}_{0}=\mathbf{N}_{0} \otimes \mathbf{I}+\mathbf{I} \otimes \mathbf{N}_{0}, \\
& (\mathbf{C} \dot{\mathbf{C}}+\dot{\mathbf{C}} \mathbf{C})_{, \dot{\mathbf{C}}}=\mathbf{C} \dot{\mathbf{C}}_{, \dot{\mathbf{C}}}+\dot{\mathbf{C}}_{, \dot{\mathbf{C}}} \mathbf{C}=\mathbf{C I}+\mathbf{I} \mathbf{C}=\mathbf{C} \otimes \mathbf{I}+\mathbf{I} \otimes \mathbf{C},\left(\mathbf{C} \dot{\mathbf{C}}+\dot{\mathbf{C}} \mathbf{C}^{2}\right)_{, \dot{\mathbf{C}}}=\mathbf{C}^{2} \otimes \mathbf{I}+\mathbf{I} \otimes \mathbf{C}^{2}, \\
& \left(\mathbf{C N}_{0}\right)_{, \dot{\mathbf{C}}}=\left(\mathbf{C}^{2} \mathbf{N}_{0}\right)_{, \dot{\mathbf{C}}}=\mathbb{O},\left(\mathbf{C N}_{0} \dot{\mathbf{C}}+\dot{\mathbf{C C N}}_{0}\right)_{, \dot{\mathbf{C}}}=\mathbf{C N}_{0} \otimes \mathbf{I}+\mathbf{I} \otimes \mathbf{C N}_{0} \quad \text { and } \\
& \Psi_{\omega, \dot{\mathbf{C}}}^{\mathrm{v}} \stackrel{\text { def }}{=} \Delta_{\omega}^{\mathrm{v}}=\sum_{\beta=1}^{12}\left(\Psi_{\omega \beta}^{\mathrm{v}} J_{\beta, \dot{\mathbf{C}}}\right) \quad \text { where } \quad \omega=1, \cdots 12 .
\end{aligned}
$$

With these, we obtain $\frac{\mathbb{A}^{\mathbf{v}}}{2 \rho_{0}}$ :

$$
\begin{aligned}
& \frac{\mathbb{A}^{\mathrm{v}}}{2 \rho_{0}}=\mathbf{I} \odot \Psi_{1, \dot{\mathbf{C}}}^{\mathrm{v}}+\dot{\mathbf{C}} \odot \Psi_{2, \dot{\mathbf{C}}}^{\mathrm{v}}+\Psi_{2}^{\mathrm{v}} \mathbb{I}+\dot{\mathbf{C}}^{-1} \odot\left(\Psi_{3}^{\mathrm{v}} J_{3}\right)_{, \dot{\mathbf{C}}}+\Psi_{3}^{\mathrm{v}} J_{3}\left(-\dot{\mathbf{C}}^{-1} \otimes \dot{\mathbf{C}}^{-1}\right) \\
& +\mathbf{N}_{0} \odot \Psi_{4, \dot{\mathbf{C}}}^{\mathrm{v}}+\boldsymbol{\Upsilon}_{\mathbf{n}_{0} \dot{\mathbf{C}}} \odot \Psi_{5, \dot{\mathbf{C}}}^{\mathrm{v}}+\Psi_{5}^{\mathrm{v}}\left(\mathbf{N}_{0} \otimes \mathbf{I}+\mathbf{I} \otimes \mathbf{N}_{0}\right)+\mathbf{C} \odot \Psi_{6, \dot{\mathbf{C}}}^{\mathrm{v}} \\
& +(\mathbf{C} \dot{\mathbf{C}}+\dot{\mathbf{C C}}) \odot \Psi_{7, \mathbf{C}}^{\mathrm{v}}+\Psi_{7}^{\mathrm{v}}(\mathbf{C} \otimes \mathbf{I}+\mathbf{I} \otimes \mathbf{C})+\mathbf{C}^{2} \odot \Psi_{8, \dot{\mathbf{C}}}^{\mathrm{v}} \\
& +\left(\mathbf{C}^{2} \dot{\mathbf{C}}+\dot{\mathbf{C}} \mathbf{C}^{2}\right) \odot \Psi_{9, \dot{\mathbf{C}}}^{\mathrm{v}}+\Psi_{9}^{\mathrm{v}}\left(\mathbf{C}^{2} \otimes \mathbf{I}+\mathbf{I} \otimes \mathbf{C}^{2}\right)+\mathbf{C N}_{0} \odot \Psi_{10, \dot{\mathbf{C}}}^{\mathrm{v}} \\
& +\left(\mathbf{C N}_{0} \dot{\mathbf{C}}+\dot{\mathbf{C}} \mathbf{C N}_{0}\right) \odot \Psi_{11, \dot{\mathbf{C}}}^{\mathrm{v}}+\Psi_{11}^{\mathrm{v}}\left(\mathbf{C N}_{0} \otimes \mathbf{I}+\mathbf{I} \otimes \mathbf{C N}_{0}\right)+\mathbf{C}^{2} \mathbf{N}_{0} \odot \Psi_{12, \dot{\mathbf{C}}}^{\mathrm{v}} \\
& =\mathbf{I} \odot \Delta_{1}^{\mathrm{v}}+\dot{\mathbf{C}} \odot \Delta_{2}^{\mathrm{v}}+\Psi_{2}^{\mathrm{v}} \mathrm{I}+\dot{\mathbf{C}}^{-1} \odot\left(\Delta_{3}^{\mathrm{v}} J_{3}+\Psi_{3}^{\mathrm{v}} J_{3, \dot{\mathbf{C}}}\right)+\Psi_{3}^{\mathrm{v}} J_{3}\left(-\dot{\mathbf{C}}^{-1} \otimes \dot{\mathbf{C}}^{-1}\right) \\
& +\mathbf{N}_{0} \odot \Delta_{4}^{\mathrm{v}}+\mathbf{\Upsilon}_{\mathbf{n}_{0} \dot{\mathbf{C}}} \odot \Delta_{5}^{\mathrm{v}}+\Psi_{5}^{\mathrm{v}}\left(\mathbf{N}_{0} \otimes \mathbf{I}+\mathbf{I} \otimes \mathbf{N}_{0}\right)+\mathbf{C} \odot \Delta_{6}^{\mathrm{v}} \\
& +(\mathbf{C} \dot{\mathbf{C}}+\dot{\mathbf{C}} \mathbf{C}) \odot \Delta_{7}^{\mathrm{v}}+\Psi_{7}^{\mathrm{v}}(\mathbf{C} \otimes \mathbf{I}+\mathbf{I} \otimes \mathbf{C})+\mathbf{C}^{2} \odot \Delta_{8}^{\mathrm{v}}+\left(\mathbf{C}^{2} \dot{\mathbf{C}}+\dot{\mathbf{C C}}^{2}\right) \odot \Delta_{9}^{\mathrm{v}} \\
& +\Psi_{9}^{\mathrm{v}}\left(\mathbf{C}^{2} \otimes \mathbf{I}+\mathbf{I} \otimes \mathbf{C}^{2}\right)+\mathbf{C N}_{0} \odot \Delta_{10}^{\mathrm{v}}+\left(\mathbf{C N}_{0} \dot{\mathbf{C}}+\dot{\mathbf{C C N}}_{0}\right) \odot \Delta_{11}^{\mathrm{v}} \\
& +\Psi_{11}^{\mathrm{v}}\left(\mathbf{C N}_{0} \otimes \mathbf{I}+\mathbf{I} \otimes \mathbf{C N}_{0}\right)+\mathbf{C}^{2} \mathbf{N}_{0} \odot \Delta_{12}^{\mathrm{v}}
\end{aligned}
$$




\subsubsection{Simplifications}

In order to define a constitutive law of our material, the corresponding Helmholtz free energy $\Psi$ is postulated with the following simplifications.

- Simplification 1: The diffusion of fluid is regarded as a determining factor for stressrelaxation phenomena of the material. Due to the limited experimental condition and many constants in the viscosity tensor, the viscous phenomenon cannot be identified separately from the diffusion. The contribution of the solid viscosity $\mathbb{A}^{\mathrm{v}}$ to the stressrelaxation is assumed to be small compared to that of the fluid diffusion and therefore neglected $\left(\Psi^{\mathrm{v}} \approx 0\right)$. Thus, the free energy (4.3.12) is reduced to

$$
\Psi=\hat{\Psi}^{\mathrm{e}}\left(\boldsymbol{\epsilon}, \mathbf{N}_{0}\right)
$$

If the solid fraction would be bigger, then the viscosity of the solid should be superposed.

- Simplification 2: Providing a stress free undistorted initial configuration, i.e. $\boldsymbol{\sigma}(\boldsymbol{\epsilon}=$ $\mathbf{0})=\mathbf{0}$, the free energy function for the transversely isotropic material is postulated:

$$
\hat{\Psi}^{\mathrm{e}}\left(I_{1}, I_{2}, I_{3}, I_{4}, I_{5}\right)=\frac{\lambda}{2} I_{1}^{2}+G_{p}\left(I_{1}^{2}-2 I_{2}\right)+\alpha I_{4} I_{1}+2\left(G_{t}-G_{p}\right) I_{5}+\frac{\beta}{2} I_{4}^{2} .
$$

Its derivative with respect to $\boldsymbol{\epsilon}$ yields the stress tensor $\boldsymbol{\sigma}$ :

$$
\begin{aligned}
\boldsymbol{\sigma} & =\frac{\partial \hat{\Psi}^{\mathrm{e}}}{\partial \boldsymbol{\epsilon}} \\
& =\lambda I_{1} \mathbf{I}+2 G_{p} \boldsymbol{\epsilon}+\alpha\left(I_{1} \mathbf{N}_{0}+I_{4} \mathbf{I}\right)+2\left(G_{t}-G_{p}\right)\left(\mathbf{N}_{0} \boldsymbol{\epsilon}+\boldsymbol{\epsilon} \mathbf{N}_{0}\right)+\beta I_{4} \mathbf{N}_{0}
\end{aligned}
$$

and the fourth-order elasticity tensor $\mathbb{A}^{\mathrm{e}}$ is

$$
\begin{aligned}
\mathbb{A}^{\mathbf{e}} & =\frac{\partial^{2} \hat{\Psi}^{\mathrm{e}}}{\partial \boldsymbol{\epsilon}^{2}} \\
& =\lambda \mathbf{I} \otimes \mathbf{I}+2 G_{p} \mathbb{I}+\alpha\left(\mathbf{I} \otimes \mathbf{N}_{0}+\mathbf{N}_{0} \otimes \mathbf{I}\right)+2\left(G_{t}-G_{p}\right)\left(\mathbf{N}_{0} \mathbb{I}+\mathbb{I N}_{0}\right)+\beta \mathbf{N}_{0} \otimes \mathbf{N}_{0}
\end{aligned}
$$

In Voigt notation it can be expressed by a $6 \times 6$ stiffness matrix for the preferred $X_{2^{-}}$ 
direction:

$$
\mathbb{C}^{\mathbf{e}}=\left[\begin{array}{cccccc}
\lambda+2 G_{p} & \lambda+\alpha & \lambda & 0 & 0 & 0 \\
\lambda+\alpha & \lambda+2 \alpha+\beta+4 G_{t}-2 G_{p} & \lambda+\alpha & 0 & 0 & 0 \\
\lambda & \lambda+\alpha & \lambda+2 G_{p} & 0 & 0 & 0 \\
0 & 0 & 0 & G_{t} & 0 & 0 \\
0 & 0 & 0 & 0 & G_{p} & 0 \\
0 & 0 & 0 & 0 & 0 & G_{t}
\end{array}\right] .
$$

- Simplification 3: In the initial condition, where the material contains no collagen type II fibers, the material is assumed to be isotropic $\left(\mathbf{N}_{0}=\mathbf{0}, \quad I_{4}=I_{5}=0\right)$. Owing to the synthesization of fibers, a preferred direction of the material $\left(\mathbf{n}_{0} \neq \boldsymbol{O}\right)$ appears and the material becomes transversely isotropic. The anisotropy of the material is regarded as a result of a remodeling effect.

- Simplification 4: Only positive activity of cells, i.e. increase of stiffness resulting from synthesization of collagen type II fibers is considered. Negative activity of cells, i.e. stiffness decrease (resorption, degradation of material) or normal activity (dead zone or lazy zone) are not included, Fig. 4.3.

\subsubsection{Diffusive-remodeled materials: IAM model}

The stress is assumed to evolve in two different time scales, a short time scale and a long time scale.

In a short time-scale (the time scale is seconds) the stress evolves depending on its actual stress and deformation. Thereby, the behavior of fluid plays an important role. Due to the squashed fluid, the stress decreases under constant compressive strain (stress relaxation) and is defined as diffusive stress.

Due to the material (collagen type-II) newly synthesized in a bioreactor the mechanical properties are modified (i.e. remodeling of solid part) in a long time-scale (the time scale is weeks). The remodeling effect causes an additional stress named remodelled stress. We assume that only the elasticity tensor evolves due to the collagen type-II and that its synthesization depends on the actual elasticity tensor and the free energy density. 


\section{Diffusive stress (in short time-scale)}

The fluid-saturated biphasic material shows relaxation of stresses in compression tests which results from the diffusion of the fluid through pores of the solid structure. It is assumed that the stress evolves in short time scale depending on the actual stress and the deformation:

$$
\dot{\mathbf{S}}^{\mathrm{d}}=\hat{\dot{\mathbf{S}}}^{\mathrm{d}}(\mathbf{S}, \mathbf{C})
$$

Stoffel et al. [76] proposed that rate of diffusive stress is proportional to the stress:

$$
\begin{aligned}
\dot{\mathbf{S}}^{\mathrm{d}} & =-D \mathbf{S}, \\
D & =\hat{D}(\mathbf{C}),
\end{aligned}
$$

where $D$ is a diffusion parameter that depends on the deformation and gives a relation between the viscous effect and the fluid motion in consequence of deformation.

If the stress is expressed in the Eulerian description, then the evolution equation is

$$
\dot{\boldsymbol{\sigma}}^{\mathrm{d}}=\operatorname{sph} \dot{\boldsymbol{\sigma}}^{\mathrm{d}}+\operatorname{dev} \dot{\boldsymbol{\sigma}}^{\mathrm{d}}=\hat{\boldsymbol{\sigma}}^{\mathrm{d}}(\boldsymbol{\sigma}, \boldsymbol{\varepsilon})=-D(\boldsymbol{\varepsilon}) \boldsymbol{\sigma}=-D(\operatorname{sph} \boldsymbol{\sigma}+\operatorname{dev} \boldsymbol{\sigma})
$$

where sph $\boldsymbol{\sigma}=\frac{1}{3} \operatorname{tr} \boldsymbol{\sigma} \mathbf{I}$ denotes the spherical part and $\operatorname{dev} \boldsymbol{\sigma}$ the deviatoric part of $\boldsymbol{\sigma}$. Moreover, we assume that the normal stress is only related with the diffusion:

$$
\dot{\boldsymbol{\sigma}}^{\mathrm{d}}=\operatorname{sph} \dot{\boldsymbol{\sigma}}^{\mathrm{d}}=-D \operatorname{sph} \boldsymbol{\sigma} \quad \text { and } \quad \operatorname{dev} \dot{\boldsymbol{\sigma}}^{\mathrm{d}}=-D \operatorname{dev} \boldsymbol{\sigma}=\mathbf{0}
$$

If the inertial force in the balance of linear momentum (B.2.3b) is neglected, the balance of linear momentum becomes

$$
\boldsymbol{O}=\operatorname{div} \boldsymbol{\sigma}+\rho \mathbf{b}
$$

Here, the stress and the body force can be split into an elastic and a diffusive part:

$$
\boldsymbol{O}=\operatorname{div} \boldsymbol{\sigma}+\rho \mathbf{b} \stackrel{\text { def }}{=} \operatorname{div} \boldsymbol{\sigma}+\boldsymbol{\pi}=\operatorname{div}\left(\boldsymbol{\sigma}^{\mathrm{e}}+\boldsymbol{\sigma}^{\mathrm{d}}\right)-\boldsymbol{\pi}^{\mathrm{e}}-\boldsymbol{\pi}^{\mathrm{d}}
$$

where $\boldsymbol{\pi}^{\mathrm{e}}$ and $\boldsymbol{\pi}^{\mathrm{d}}$ are the elastic and diffusive part of the volumetric force, respectively.

The elastic and diffusive volumetric forces are assumed to be separated from each other and 
the diffusive volumetric force, $\boldsymbol{\pi}^{\mathrm{d}}$, induced by the diffusion of fluid through the pores explains the stress relaxation curve by compression test:

$$
\begin{aligned}
\boldsymbol{\pi}^{\mathrm{d}} & =\operatorname{div} \boldsymbol{\sigma}^{\mathrm{d}}=\operatorname{div} \int \dot{\boldsymbol{\sigma}}^{\mathrm{d}} d t=-\operatorname{div} \int(D \operatorname{sph} \boldsymbol{\sigma}) d t=-\int \operatorname{div}(D \operatorname{sph} \boldsymbol{\sigma}) d t \\
& =-\int(\operatorname{grad} D \operatorname{sph} \boldsymbol{\sigma}+D \operatorname{div} \operatorname{sph} \boldsymbol{\sigma}) d t .
\end{aligned}
$$

With the further assumption that the material is homogenous with respect to the diffusion parameter $D, \operatorname{grad} D=\boldsymbol{O}$, the above equation becomes

$$
\begin{aligned}
\boldsymbol{\pi}^{\mathrm{d}} & =-\int D \operatorname{div} \operatorname{sph} \boldsymbol{\sigma} d t=-\int D \operatorname{div}\left(\frac{1}{3} \operatorname{tr} \boldsymbol{\sigma} \mathbf{I}\right) d t=-\frac{1}{3} \int D \operatorname{div}(\operatorname{tr} \boldsymbol{\sigma} \mathbf{I}) d t \\
& =-\frac{1}{3} \int D(\mathbf{I} \operatorname{grad} \operatorname{tr} \boldsymbol{\sigma}+\operatorname{tr} \boldsymbol{\sigma} \operatorname{div} \mathbf{I}) d t=-\frac{1}{3} \int D \operatorname{grad} \operatorname{tr} \boldsymbol{\sigma} d t \mathbf{I} \\
& =-\frac{1}{3} \int D \operatorname{grad} \operatorname{tr} \boldsymbol{\sigma} d t=-\frac{1}{3} \int D \operatorname{grad} \operatorname{tr}\left(\boldsymbol{\sigma}^{\mathbf{s}}+\boldsymbol{\sigma}^{\mathbf{f}}\right) d t .
\end{aligned}
$$

Here, $\boldsymbol{\sigma}^{\mathbf{s}}$ is the stress of the solid and $\boldsymbol{\sigma}^{\mathrm{f}}$ the stress of the fluid.

With the assumption that the fluid stress in the deformed configuration is the hydrostatic pressure $p$ :

$$
\boldsymbol{\sigma}^{\mathrm{f}}=-p \mathbf{I}
$$

the diffusive volumetric force due to fluid $\boldsymbol{\pi}^{\mathrm{df}}$ can be estimated:

$$
\begin{aligned}
\boldsymbol{\pi}^{\mathrm{df}} & =-\frac{1}{3} \int D \operatorname{grad} \operatorname{tr} \boldsymbol{\sigma}^{\mathrm{f}} d t=-\frac{1}{3} \int D \operatorname{grad}\left(\boldsymbol{\sigma}^{\mathrm{f}}: \mathbf{I}\right) d t \\
& =\frac{1}{3} \int D \operatorname{grad}(p \mathbf{I}: \mathbf{I}) d t=\int D \operatorname{grad} p d t
\end{aligned}
$$

In case of the unconfined compression test (compression in $x_{2}$-direction, in $x_{1}-$ and $x_{3}-$ directions are not confined, Fig. 5.14 ), the volumetric force reads

$$
\boldsymbol{\pi}^{\mathrm{df}}=\pi_{i}^{\mathrm{df}} \boldsymbol{e}_{i}=\int D p_{, i} d t \boldsymbol{e}_{i}
$$

and the force in compression direction due to the diffusion is

$$
\pi_{2}^{\mathrm{df}}=\int D \frac{\partial p}{\partial x_{2}} d t
$$


Especially, $D$ is assumed to be a function of a volumetric strain $\varepsilon_{\mathrm{V}}\left(=\varepsilon_{i i}=\varepsilon_{11}+\varepsilon_{22}+\varepsilon_{33}\right)$ in order to describe deformation-dependent diffusion:

$$
D=\hat{D}\left(\varepsilon_{\mathrm{v}}\right)=D_{0}+D_{1} \varepsilon_{\mathrm{V}}
$$

where $D_{0}$ and $D_{1}$ are material parameters describing the relation of volume change and the stress relaxation over time during the compression test.

\section{Remodeled stress (in long time-scale)}

The newly synthesized material (collagen type-II) in the bioreactor changes the material properties in a long time scale, and it is postulated that only the elasticity tensor $\mathbb{A}^{e}$ changes as a function of the actual elasticity and the free energy density, and it has its own critical value:

$$
\dot{\mathbb{A}}^{\mathrm{e}}=\hat{\dot{\mathbb{A}}}^{\mathrm{e}}\left(\mathbb{A}^{\mathrm{e}}, \Psi\right), \quad \text { where } \quad \mathbb{A}_{0}^{\mathrm{e}} \leq \mathbb{A}^{\mathrm{e}} \leq \mathbb{A}_{\text {crit }}^{\mathrm{e}} .
$$

where $\mathbb{A}_{0}^{\mathrm{e}}$ is an initial elasticity and $\mathbb{A}_{\text {crit }}^{\mathrm{e}}$ is its critical value.

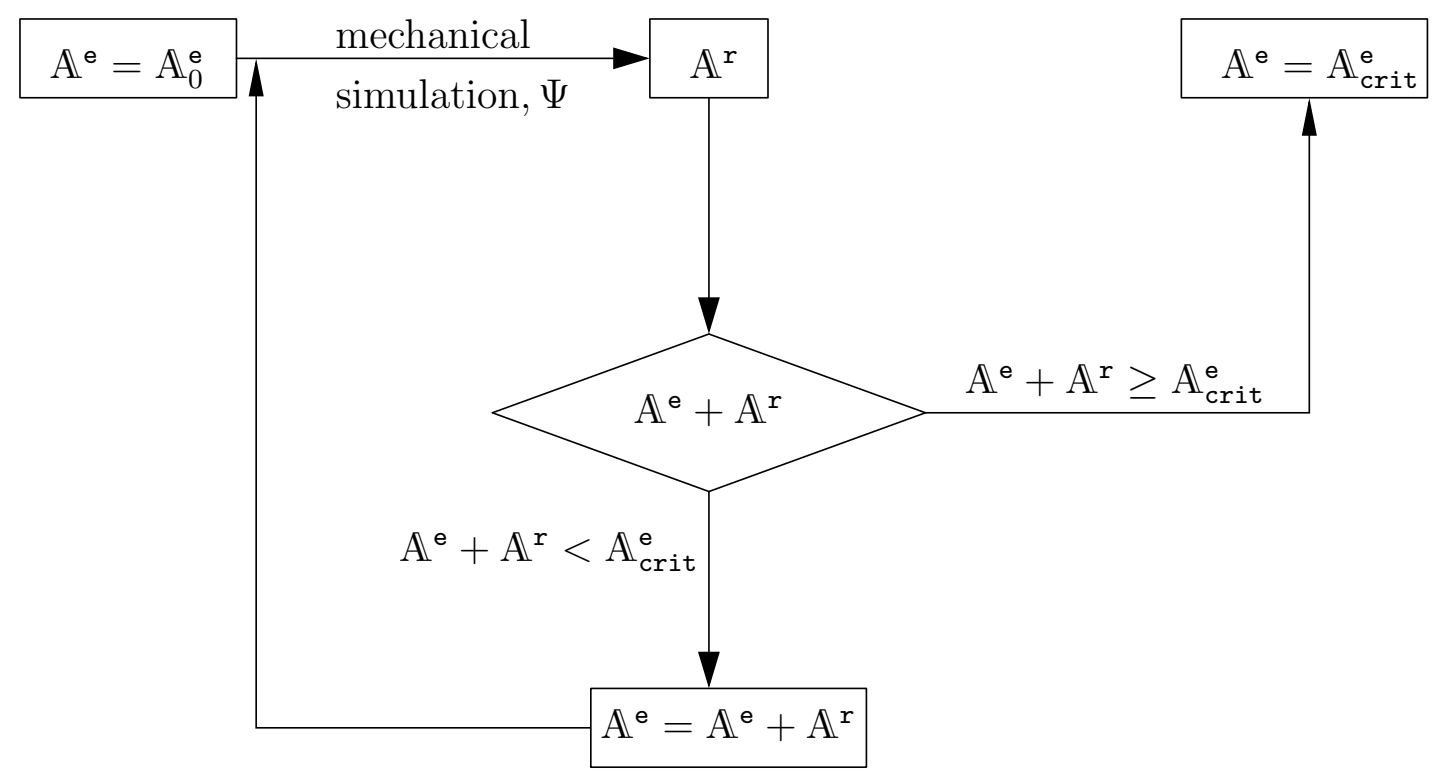

Figure 4.4: Flowchart of elasticity remodeling

Further, the following evolution law of the stiffness is proposed:

$$
\dot{\mathbb{A}}^{\mathrm{e}}=k \sqrt[n]{\Psi}\left(\mathbb{A}_{\text {crit }}^{\mathrm{e}}-\mathbb{A}^{\mathrm{e}}\right), \quad \Psi=\hat{\Psi}(\mathbf{C})
$$


where $k$ and $n$ are remodeling parameters describing the remodeling velocity, [77] and [87].

The physical meaning of the Eq. (4.3.75) is that the stiffness tensor evolves due to the mechanical stimulus up to a critical value that is obtained at the end of stimulation in a bioreactor.

\subsubsection{Summary of the IAM model}

From Section 4.3.3 and Section 4.3.4, we propose the following model for the cell-seeded condensed collagen gel material on the basis of the diffusive-remodelled elastic phenomenon.

The total stress rate is

$$
\dot{\boldsymbol{\sigma}}=\dot{\boldsymbol{\sigma}}^{\mathrm{e}}+\dot{\boldsymbol{\sigma}}^{\mathrm{d}}
$$

Its elastic part is

$$
\sigma^{\mathrm{e}}=\mathbb{A}^{\mathrm{e}}: \boldsymbol{\epsilon}
$$

The rate of elastic stress is

$$
\dot{\sigma}^{\mathrm{e}}=\frac{\cdot}{\mathbb{A}^{\mathrm{e}}: \boldsymbol{\epsilon}}=\dot{\mathbb{A}}^{\mathrm{e}}: \boldsymbol{\epsilon}+\mathbb{A}^{\mathrm{e}}: \dot{\boldsymbol{\epsilon}} \approx \mathbb{A}^{\mathrm{e}}: \dot{\boldsymbol{\epsilon}}
$$

where $\dot{\mathbb{A}}^{\mathrm{e}} \approx \mathbb{O}$ in short time-scale. $\mathbb{A}^{\mathrm{e}}$ evolves in long time-scale according to Eq. (4.3.75).

The stress evolves in short time-scale (compression test) as a function of the volumetric strain.

From Eqs. (4.3.64) and (4.3.73), the following stress is added

$$
\dot{\boldsymbol{\sigma}}^{\mathrm{d}}=-\frac{D_{0}+D_{1} \varepsilon_{\mathrm{v}}}{3} \sigma_{i i} \mathbf{I}
$$

which explains the stress relaxation due to fluid behavior. 


\subsubsection{Comparison of KLM model and IAM model}

Kuei, Lai, and Mow (KLM) regarded the interaction between the solid and fluid phase as a determining factor for the mechanical behavior of a biphasic tissue. Thus, the permeability plays an important role, how much fluid flow in and out of the porous material according to external force.

They modeled articular cartilage as a homogeneous binary mixture of an incompressible, isotropic, linearly elastic solid and an incompressible, inviscid fluid (KLM model) [55].

The constitutive equations are given by

$$
\begin{array}{ll}
\text { solid phase: } & \boldsymbol{\sigma}^{\mathbf{s}}=-\alpha p \mathbf{I}+\lambda^{\mathbf{s}} \operatorname{tr} \boldsymbol{\varepsilon}+2 \mu^{\mathbf{s}} \boldsymbol{\varepsilon}, \\
\text { fluid phase: } & \boldsymbol{\sigma}^{\mathrm{f}}=-p \mathbf{I},
\end{array}
$$

where superscript $\mathrm{s}$ stands for the solid phase and $\mathrm{f}$ for fluid. $\boldsymbol{\sigma}$ and $\boldsymbol{\varepsilon}$ are the stress and strain tensor, respectively. $p$ is the pressure, $\alpha$ is the solid content defined by the ratio of solid volume to fluid volume $\left(\alpha \stackrel{\text { def }}{=} V^{\mathbf{s}} / V^{\mathbf{f}}\right), \lambda^{\mathbf{s}}$ and $\mu^{\mathbf{s}}$ are Lamé constants for solid phase.

The balance of linear momentum (B.2.3b) is

$$
\begin{array}{ll}
\text { solid phase: } & \rho^{\mathbf{s}} D_{t}^{\mathbf{s}} \boldsymbol{v}^{\mathbf{s}}=\operatorname{div} \boldsymbol{\sigma}^{\mathbf{s}}+\rho^{\mathbf{s}} \mathbf{b}^{\mathbf{s}}, \\
\text { fluid phase: } & \rho^{\mathrm{f}} \mathrm{D}_{t}^{\mathrm{f}} \boldsymbol{v}^{\mathrm{f}}=\operatorname{div} \boldsymbol{\sigma}^{\mathrm{f}}+\rho^{\mathrm{f}} \mathbf{b}^{\mathrm{f}},
\end{array}
$$

where $\boldsymbol{v}^{\mathbf{s}} / \mathbf{f}$ is velocity of the solid/fluid phase and $D_{t}^{\mathbf{s} / \mathbf{f}}$ is the material time derivative with respect to solid/fluid phase. Under quasi-static conditions, the inertial force can be neglected:

$$
\begin{aligned}
& \text { solid phase: } \rightarrow \boldsymbol{O}=\operatorname{div} \boldsymbol{\sigma}^{\mathrm{s}}+\rho^{\mathrm{s}} \mathbf{b}^{\mathrm{s}}=\operatorname{div} \boldsymbol{\sigma}^{\mathrm{s}}-\boldsymbol{\pi} \\
& \text { fluid phase: } \rightarrow \boldsymbol{O}=\operatorname{div} \boldsymbol{\sigma}^{\mathrm{f}}+\rho^{\mathrm{f}} \mathbf{b}^{\mathrm{f}}=\operatorname{div} \boldsymbol{\sigma}^{\mathrm{f}}+\boldsymbol{\pi},
\end{aligned}
$$

where the force interacting between the two phases is assumed to be the volumetric force $\boldsymbol{\pi}$ which is the diffusive body force. It can be derived from Darcy's law as follows.

The Darcy's law states the flow rate $\mathbf{Q}\left(\mathrm{m}^{3} / \mathrm{s}\right)$ through a porous solid is proportional to the permeability $k\left(\mathrm{~m}^{2}\right)$, the gradient of the pressure $P$ applied to the body and the area $A$, and inversely proportional to the dynamic viscosity $\mu(\mathrm{Pa} \cdot \mathrm{s})$ :

$$
\mathbf{Q}=-\frac{k A}{\mu} \operatorname{grad} P=-\frac{A}{K} \operatorname{grad} P \quad \text { or } \quad \mathbf{q}=\frac{\mathbf{Q}}{A}=-\frac{k}{\mu} \operatorname{grad} P=-\frac{1}{K} \operatorname{grad} P,
$$


where $\mathbf{q}(\mathrm{m} / \mathrm{s})$ is the flux of discharge per unit area and $K\left(=\frac{\mu}{k}, \mathrm{Ns}_{\mathrm{m}} \mathrm{m}^{4}\right)$ is the constant diffusive drag coefficient.

The formula above can be adjusted to the model. The flow rate per unit area $\mathbf{q}(=\mathbf{Q} / A)$ is the velocity of fluid relative to solid:

$$
\mathbf{q}=\boldsymbol{v}^{\mathrm{fs}}=\boldsymbol{v}^{\mathrm{f}}-\boldsymbol{v}^{\mathrm{s}}=-\frac{\operatorname{grad} p}{K} \rightarrow K\left(\boldsymbol{v}^{\mathrm{s}}-\boldsymbol{v}^{\mathrm{f}}\right)=\operatorname{grad} p
$$

where $p$ is the hydrostatic pressure.

If we define $\operatorname{grad} p$ as a force per volume $\boldsymbol{\pi}$ :

$$
\boldsymbol{\pi} \stackrel{\text { def }}{=} \operatorname{grad} p=K\left(\boldsymbol{v}^{\mathbf{s}}-\boldsymbol{v}^{\mathbf{f}}\right)
$$

it has a physical meaning that a force per volume depends on the permeability $k$, the dynamic viscosity $\mu$ and the relative motion of fluid $\boldsymbol{v}^{\mathrm{fs}}$. For example, the more volumetric force is caused with a bigger dynamic viscosity or with a bigger relative velocity of fluid or a smaller permeability. On the contrary, the higher porosity means the higher permeability and the smaller diffusive drag coefficient and the diffusive force due to fluid behavior becomes smaller.

The continuity equations are

$$
\begin{array}{ll}
\text { solid phase: } & D_{t} \rho^{\mathbf{s}}+\rho^{\mathbf{s}} \operatorname{div} \boldsymbol{v}^{\mathbf{s}}=0, \\
\text { fluid phase: } & \mathrm{D}_{t} \rho^{\mathrm{f}}+\rho^{\mathrm{f}} \operatorname{div} \boldsymbol{v}^{\mathrm{f}}=0 .
\end{array}
$$

With an assumption of incompressibility of solid and fluid phase $\left(D_{t} \rho^{\mathrm{s} / \mathrm{f}}=0\right)$ it can be simplified:

$$
\begin{array}{ll}
\text { solid phase: } \rightarrow \operatorname{div} \boldsymbol{v}^{\mathrm{s}}=0, \\
\text { fluid phase: } \rightarrow \operatorname{div} \boldsymbol{v}^{\mathrm{f}}=0 .
\end{array}
$$

In consideration of the solid content $\alpha$, the following continuity equation governing the binary mixture of an incompressible solid and an incompressible fluid is obtained:

$$
\operatorname{div} \boldsymbol{v}^{\mathrm{f}}+\alpha \operatorname{div} \boldsymbol{v}^{\mathbf{s}}=0
$$


In terms of body force, we can compare the KLM model and the IAM model proposed in Section 4.3.4.

The body force $\boldsymbol{\pi}$ of the KLM model, Eq. (4.3.85), is a volumetric force that is related with the fluid flow. In the IAM model, the fluid flow is considered in a volumetric strain $\varepsilon_{\mathrm{v}}$ on which the diffusion parameter $D$ depends. Therefore, the diffusive volumetric force due to fluid $\boldsymbol{\pi}^{\mathrm{df}}$ of the IAM model, Eq. (4.3.70), corresponds to the body force $\boldsymbol{\pi}$ of the KLM model:

$$
\boldsymbol{\pi}=K\left(\boldsymbol{v}^{\mathrm{s}}-\boldsymbol{v}^{\mathrm{f}}\right) \stackrel{!}{=} \boldsymbol{\pi}^{\mathrm{df}}=\int\left(D_{0}+D_{1} \varepsilon_{\mathrm{V}}\right) \operatorname{grad} p d t
$$

Let us think a special case that there is no relative motion of fluid with respect to solid $\left(\boldsymbol{v}^{\mathrm{f}}=\boldsymbol{v}^{\mathrm{s}}\right)$. The KLM model gives, there is no body force $(\boldsymbol{\pi}=\boldsymbol{O})$ and the IAM model also gives $\boldsymbol{\pi}^{\mathrm{df}}=\boldsymbol{O}$, as no relative motion of fluid means $\operatorname{grad} p=\boldsymbol{O}$. 


\section{Chapter 5}

\section{Experiments}

\subsection{Cell-seeded condensed collagen gel}

The preparation of specimens explained in this section and the histologies described in the next section were carried out in the Department for Orthopaedics and Trauma Surgery, University Hospital Aachen.

Collagen type I matrix system is important as a cartilage repair material.

Most of the established matrix systems [61] use collagen type I of bovine origin seeded in high cell densities. In contrast, we use collagen type I gel made of rat tail collagen (CaReS, Arthro Kinetics, Esslingen, Germany) for the cultivation of human chondrocytes in low cell densities [31].

After seeding the collagen type I gel with human chondrocytes with a cell density of ca. 2 . $10^{5}$ cells $/ \mathrm{ml}$, the collagen is condensed by weights (between $2-4 \mathrm{~kg}$ ) in a compression chamber for $6-12$ hrs, Fig. 5.1. When the cell-seeded collagen type I gel is completely consolidated, the specimens are manufactured with stamping of a circular cutting blade. 


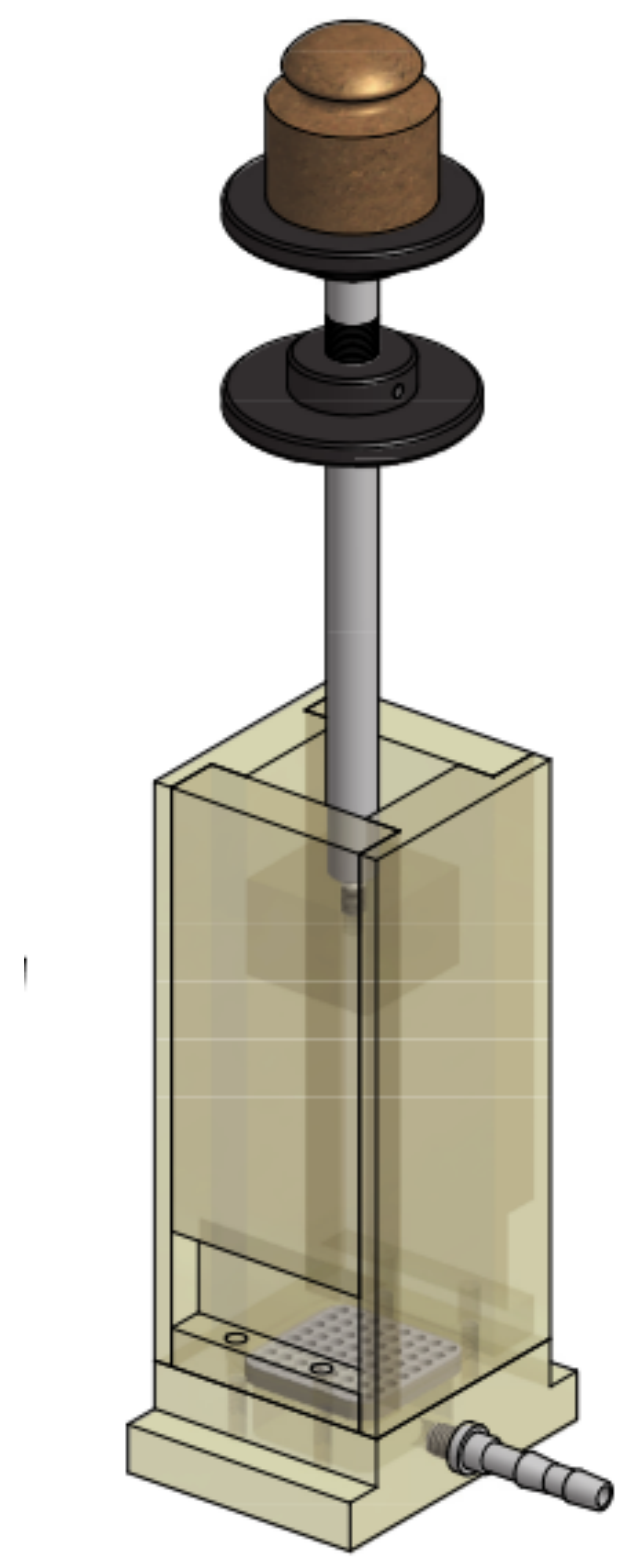

Figure 5.1: Chamber for cell-seeded condensed collagen type I matrix, [63] 
Due to the restrictions imposed by the bioreactor, the diameter of a specimen is $10 \mathrm{~mm}$ and the thickness $2-3 \mathrm{~mm}$.

Figure 5.2 shows photos of specimens from the cell-seeded condensed collagen gel type I matrix, a saturated and a dried one. The solid state possesses less than $10 \%$ of the whole weight and the fluid possesses more than $90 \%$.

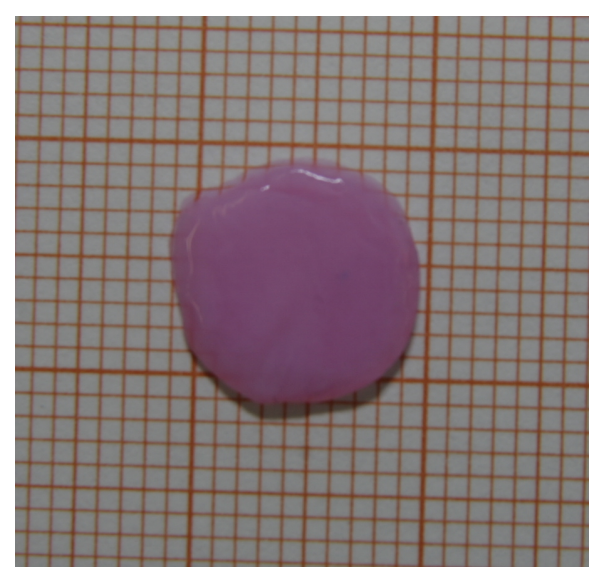

(a) Saturated (210 mg)

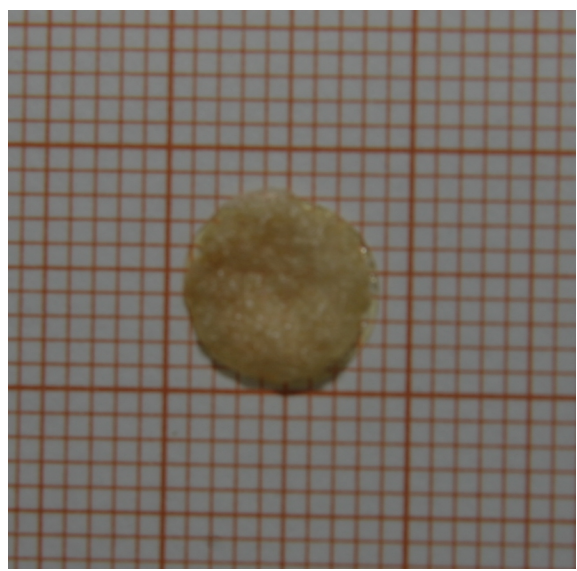

(b) Dried (16 mg)

Figure 5.2: Cell-seeded condensed collagen type I matrix 


\subsection{Bioreactor}

\subsubsection{Bioreactor system}

Bioreactors are developed in order to stimulate cell-seeded condensed collagen gel type I mechanically [75].

Our bioreactor is composed of four parts, see Fig. 5.3: a support with loading device (Part 1), a cultivation cylinder (Part 2), a rod for load application (Part 3) and a loading device (Part 4). Before running a bioreactor, all the parts are sterilized [78].

The cell-seeded specimens as presented in Section 5.1 are put on a slot in a cylindrical chamber and soaked in nutrient medium under sterile condition (Part 2). After the cylinder is screwed on, the bioreactor system is put together and ready to run in non-sterile conditions. Gas exchange is also possible through two membrane ventilators (the blue parts in the Fig. 5.4).

Rotation of a motor of the loading device (Part 4) drives the vertical rod (Part 3) up and down periodically with a frequency of ca. $1 / 3 \mathrm{~Hz}$ via an excentric. The periodic moving rod compresses the specimen by ca. one third of its thickness. In order to prevent germs intrusion, a rubber coat covers the guideway between rod and cylinder chamber (Part 3). During the mechanical stimulation, the nutrient medium is squeezed out when compressed and soaked in when released, which makes cells more active than the case of not-mechanically stimulated cells.

The vertical forces are measured directly by the load cell (Part 1) which is fixed under the specimen. The bioreactor runs in an incubator at a constant temperature of $37^{\circ} \mathrm{C}$ for four weeks, which gives an acceptable explanation for the assumption of isothermal process in Subsection 4.3.1. 


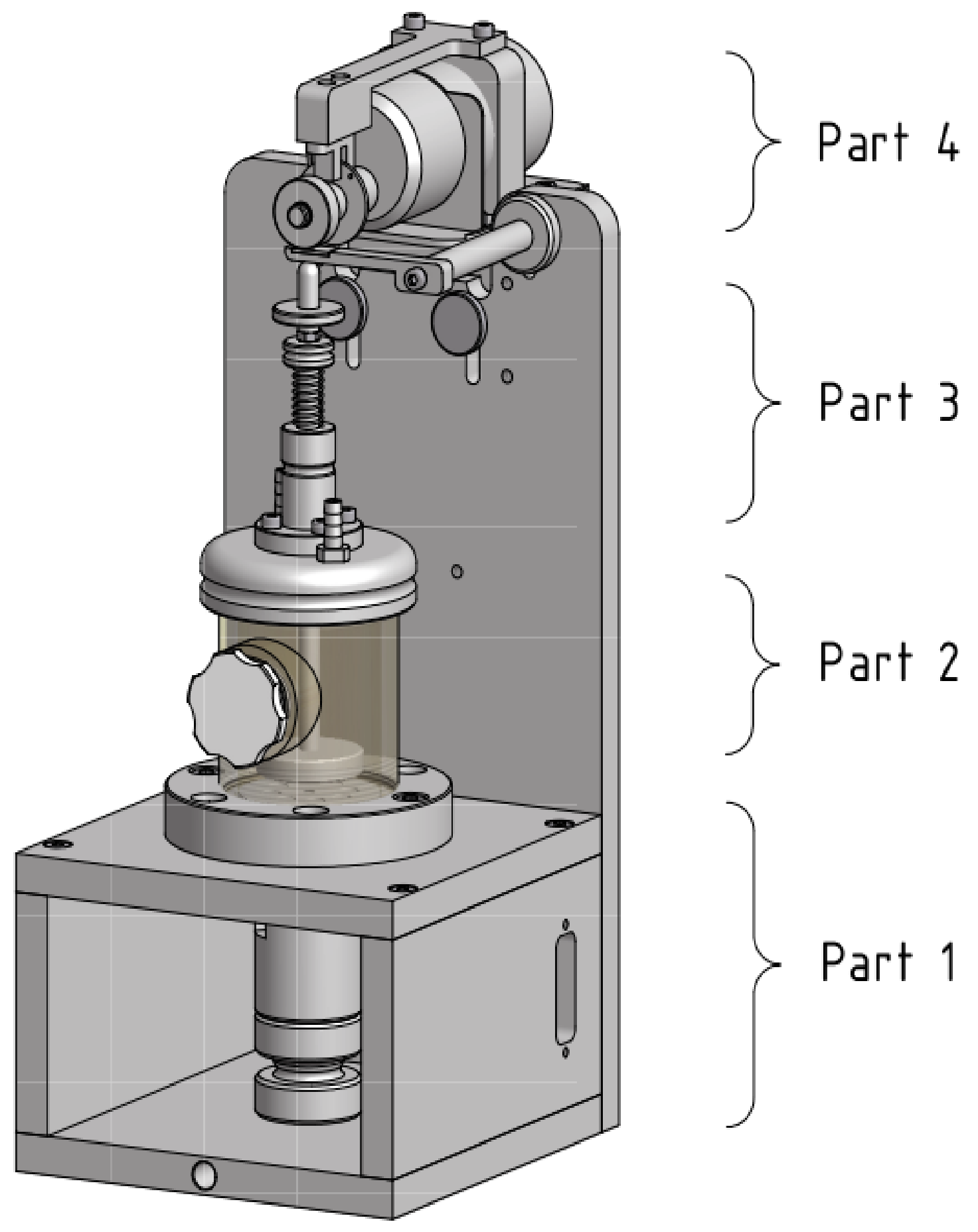

Figure 5.3: Drawing of a bioreactor 


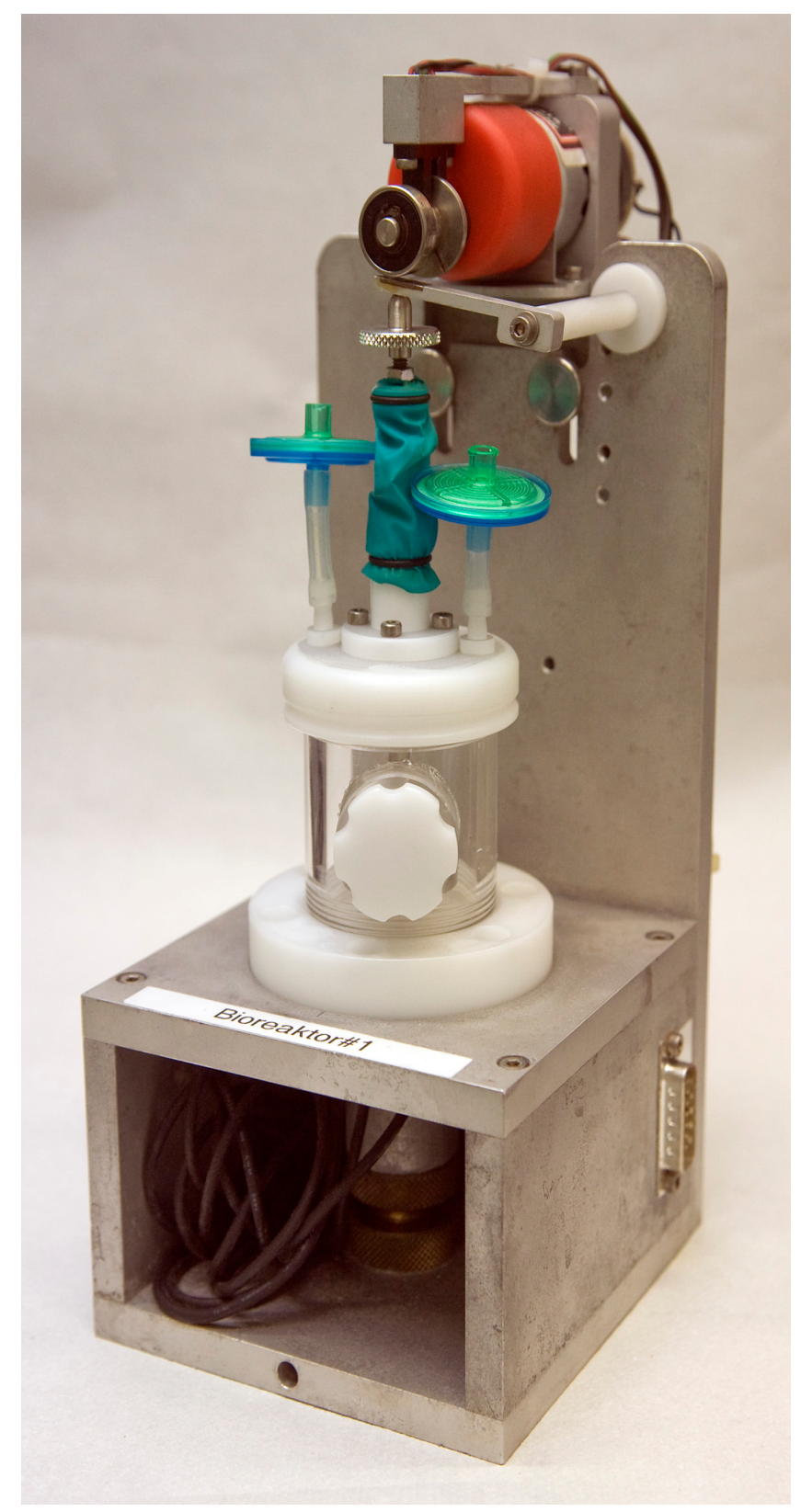

Figure 5.4: A photo of a bioreactor 
The following histological sections Fig. 5.5 show the efficiency of the bioreactor.

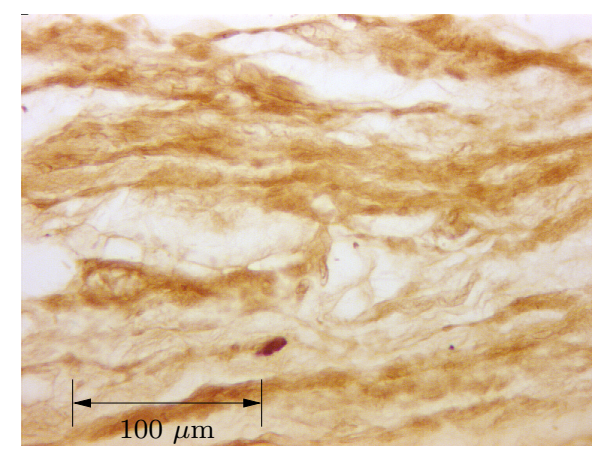

(a) Stimulated

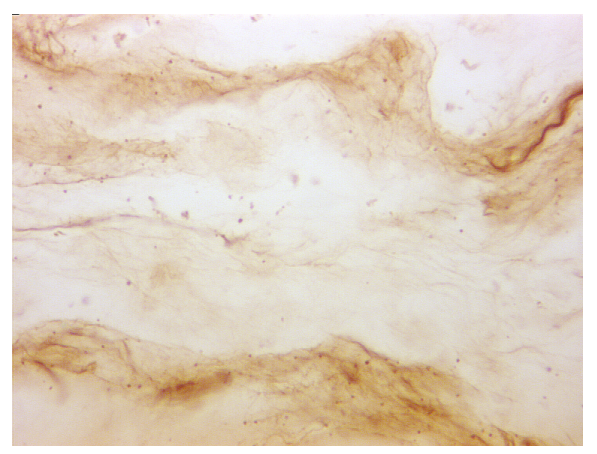

(b) Not-stimulated

Figure 5.5: Comparison of mechanically stimulated and not-stimulated specimens

The histology of the specimen stimulated in a bioreactor for four weeks shows more synthesized collagen type II fiber than that of not-stimulated specimen. Its fiber layers are discernible, more and thicker. On the other hand, the specimen not mechanically stimulated but supplied only with nutrition synthesize collagen type II fiber less than that and its layers are indistinguishable. A quantitative comparison can be made only through copression tests, Section 5.4. 


\subsubsection{Phenomena occuring in a bioreactor}

During running the bioreactor, the seeded cells (chondrocytes in collagen type I) consume the nutrient and synthesize collagen type II fibers which enhance the mechanical properties of the tissue, as Fig. 5.6 illustrates.

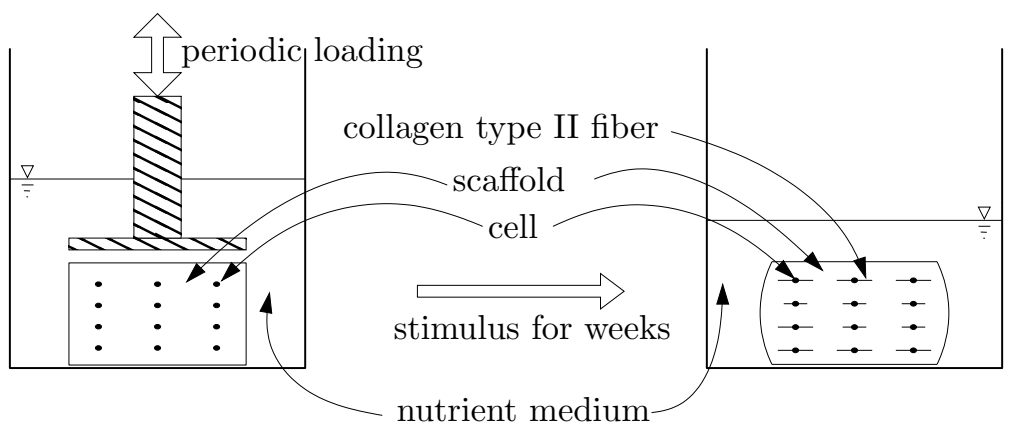

Stimulation
After stimulation

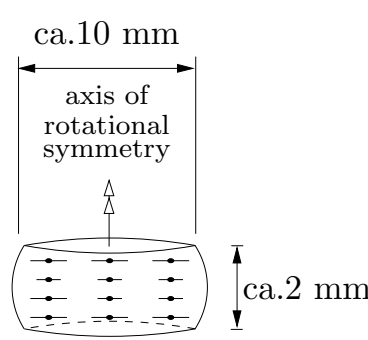

Cylindrical specemen with collagen type II

Figure 5.6: Sketch of a bioreactor

Before the stimulation in a bioreactor, a specimen contains cells and collagen type I, but after four weeks of mechanical stimulation, newly synthesized collagen type II fibers are also observed. The more synthesized collagen type II fibers appear the stiffer the specimen become.

The following photos are histologies of the specimen photographed by Karsten Gavénis [31], [61].

In Fig. 5.7, the dark brown layers are newly synthesized collagen type II fibers. Their direction is perpendicular to the loading direction and they form layers.

By comparison, the histologies of the specimen (see Fig. 5.7 (b)) supplied with nutrition, but not mechanically stimulated, do not show discernible layers. The initial state containing only chondrocytes and collagen type I shows faint histologies, Fig. 5.7 (a).

These comparisons are only eye measure and can be quantitatively measured through compression test, Section 5.4. 

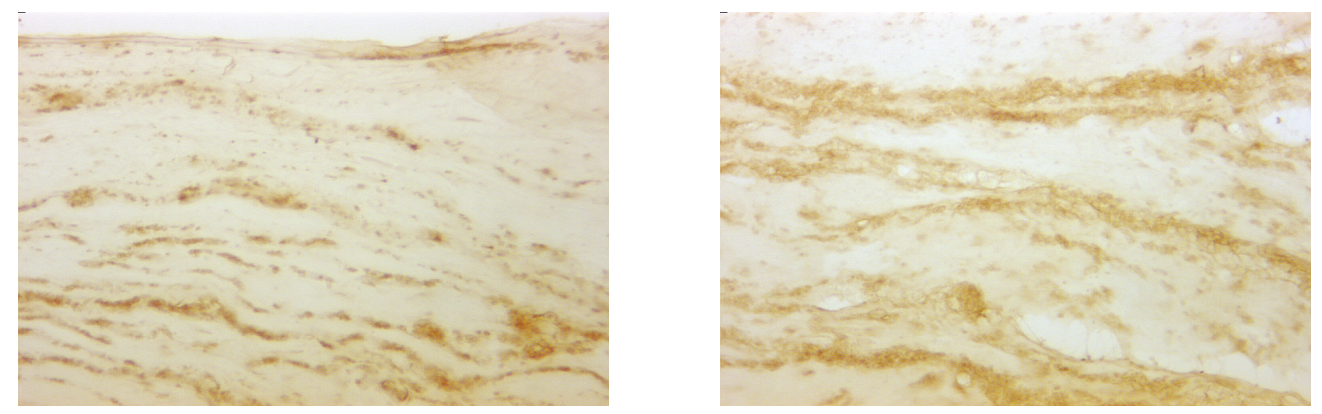

(a) Initial state
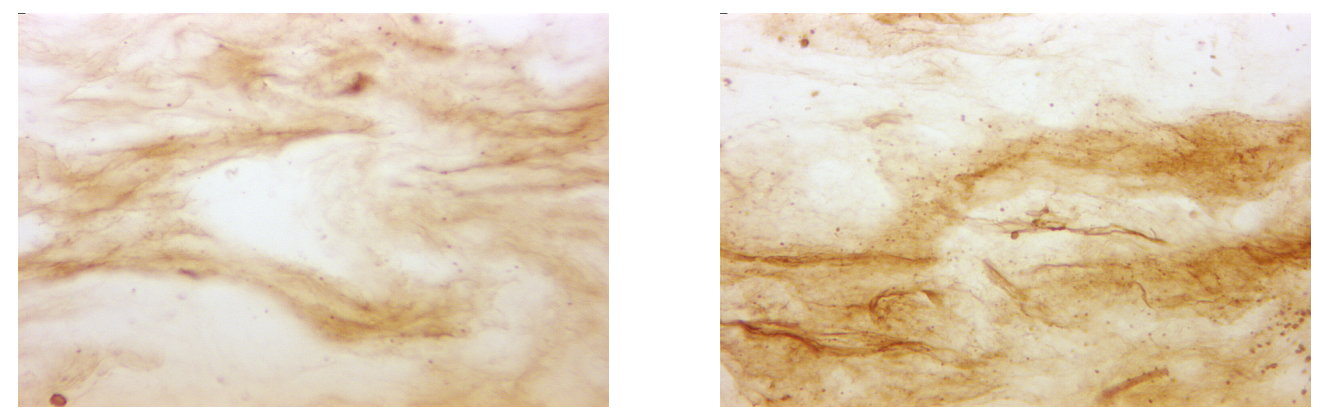

(b) After 4 weeks in nutrient medium without stimulation

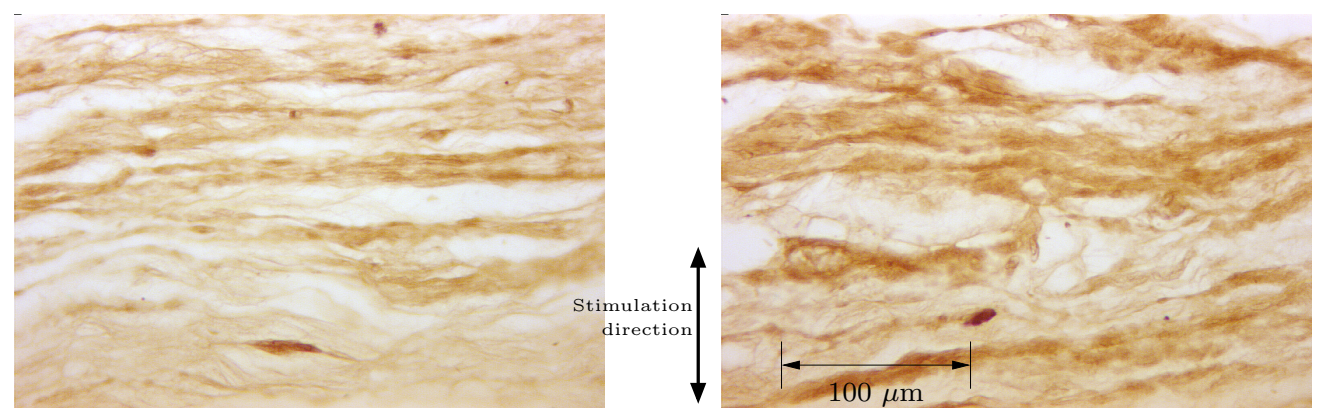

(c) After 4 week-bioreactor stimulation

Figure 5.7: Histologies after 4 weeks stimulation in a bioreactor 


\subsubsection{Signals from bioreactors}

Figure 5.8 illustrates signals from the bioreactors. The upper green line and the lower red line respectively represent the rod rotations (stimulations) and the signals from the load cell which measures the reaction forces of the specimens according to the stimulation. Even though the compression depth of specimen cannot be measured, we can see whether the specimens are correctly stimulated, by checking whether the reaction forces correspond to the stimulus.
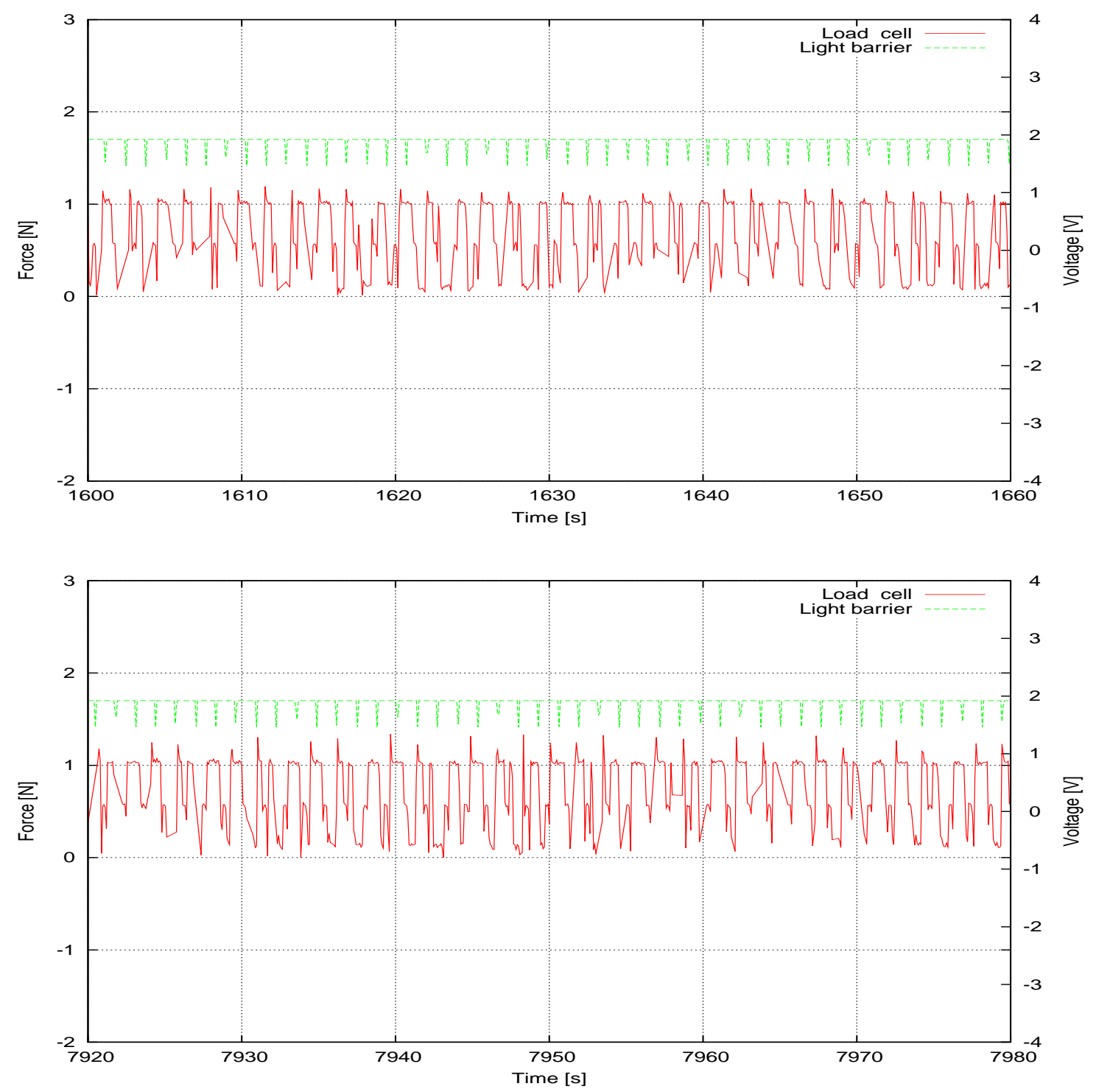

Figure 5.8: Signals from a bioreactor, at first day

The above two figures in Fig. 5.8 were recorded at the first day of a bioreactor stimulation. Even though the cell-seeded condensed collagen gel was already condensed in a compression 
chamber, the material looks instable at the beginning of bioreactor stimulation and it becomes thinner.

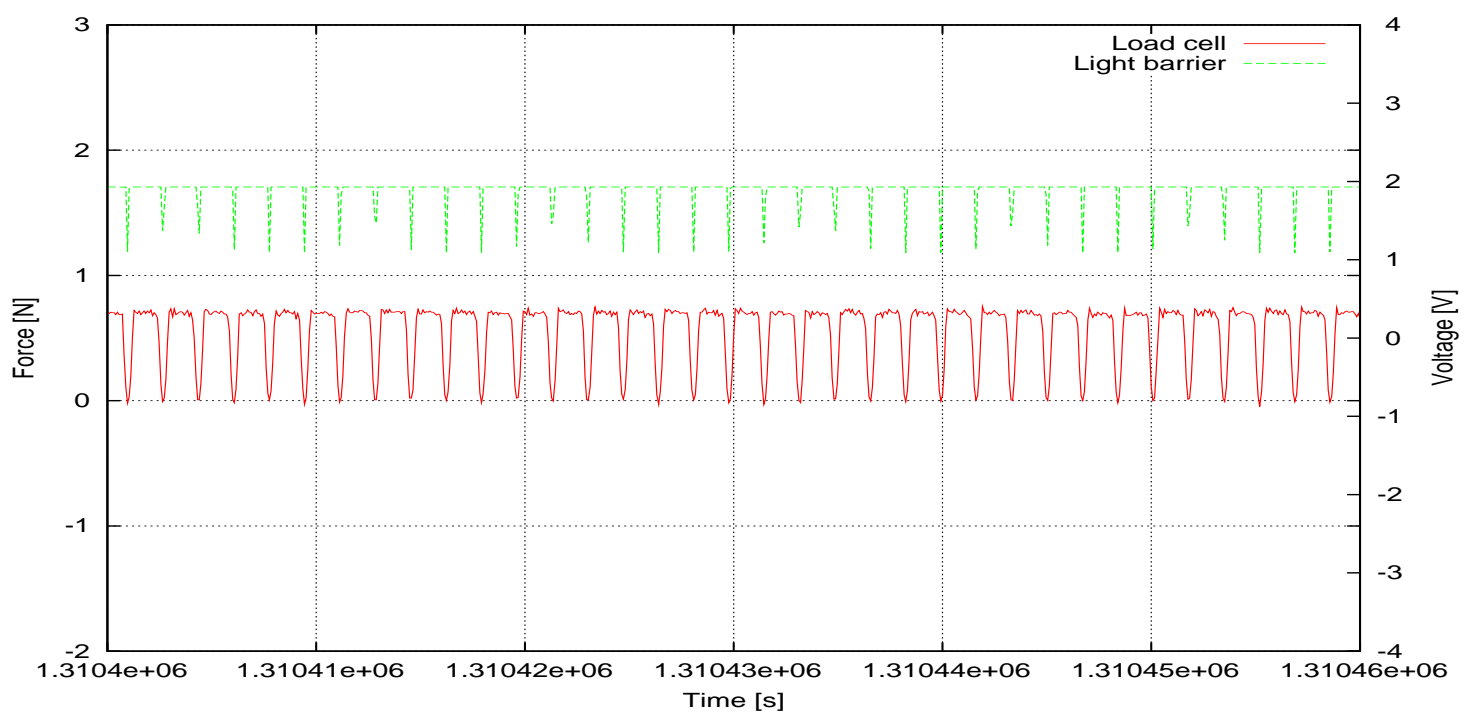

Figure 5.9: Signals from a bioreactor, at sixteenth day

The force signal recorded at the sixteenth day, shown in Fig. 5.9, looks stable compared to the ones recorded at the first day. But the periodic stimulation (compression) in a bioreactor condense the specimens, which leads to decrease of the thickness.

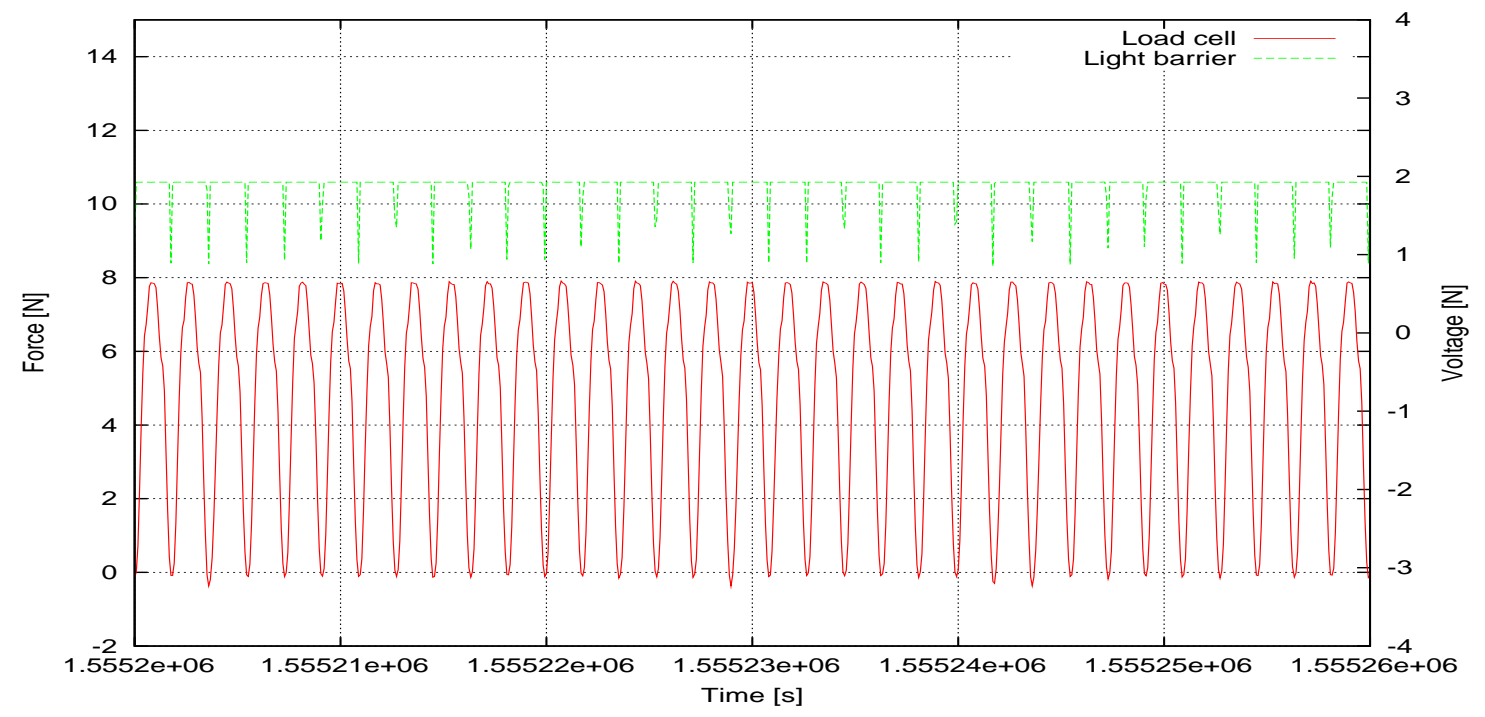

Figure 5.10: Signals from a bioreactor, at nineteenth day

As the thickness of the specimen becomes smaller, the reaction force signal becomes weak, which results in insufficient stimulation. Therefore, the rod should be pulled downward several 
times during the bioreactor runs in order to stimulate adequately. As the adjustment of the rod position is carried out manually - a rule of thumb, it is very difficult to keep a constant stimulation (force). Figure 5.10 shows the signals after adjusting the rod position. The bigger force on the nineteenth day than that at sixteenth day, Fig. 5.9, doesn't mean that the stiffness of the specimen increases but the origin of the rod is lowered.

As the signals from bioreactors are not sufficient for stating material properties, mechanical tests should be performed, Section 5.3. If we perform mechanical test every week, we can follow changes of material properties (remodeling effects) that are arisen due to cell activity. 


\subsection{Mechanical tests for soft tissues}

Mechanical properties of soft tissues can be determined by tension, pure shear, compression, bending, and torsion tests. Among them the first three tests are the most relevant tests, Gao et al. [27] and Miller [57].

Uniaxial tension test is very popular for elastomer. The specimen must be much longer in the direction of stretching than in the width and thickness dimensions, left Fig. 5.11. There should be no lateral constraint to specimen thinning.

Pure shear experiment looks like a very wide uniaxial tension test, middle Fig. 5.11. While a specimen is stretched in its longitudinal direction in the case of an uniaxial tensile test, a specimen is loaded in its lateral direction in the pure shear test. Then, a state of pure shear exists in the specimen at a 45 degree angle to the stretching direction. The most significant aspect of the specimen is that it is much shorter in the direction of stretching than the width. The specimen is perfectly constrained in the lateral direction such that all specimen thickening occurs in the thickness direction. There is no rigid body rotation of the overall material element as it deforms but the axial compression of the grips induces only the shearing motion.

Compression test, right Fig. 5.11, is widely used for soft tissue such as articular cartilage and meniscus, for they are mainly compressed during walking motion in vivo.

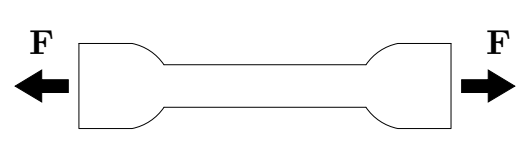

Tension test specimen

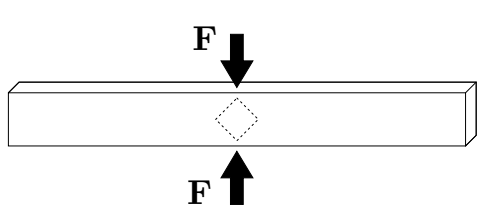

Pure shear test specimen

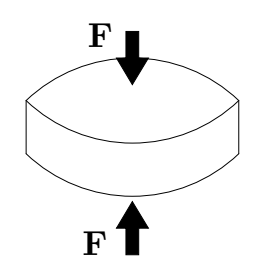

Compression test specimen

Figure 5.11: Specimens for uniaxial tension test and compression test 
There are three kinds of compression test: unconfined compression, confined compression, and indentation test.

In unconfined compression test, left Fig. 5.12, a tissue specimen is compressed between impermeable plate and impermeable stamp to a predefined stress or strain. Due to the impermeability boundary condition, interstitial fluid flows out only in lateral direction.

In confined compression test, middle Fig. 5.12, a soft tissue specimen is placed in a impermeable chamber and compressed with a permeable stamp. Due to the boundary condition of impermeable plate, the interstitial fluid flows into pores of the stamp. In both unconfined and confined tests, tissue specimen should be cut out.

But in indentation test, right Fig. 5.12, a tissue specimen does not need to be cut out. It is compressed with a plane- or spherical-ended cylindrical indenter whose cross section area is smaller than that of tissue. In the test, the interstitial fluid flows out in any direction except for the case of impermeable indenter. The test can be particularly used for tissue in vivo, i.e. in surgery.

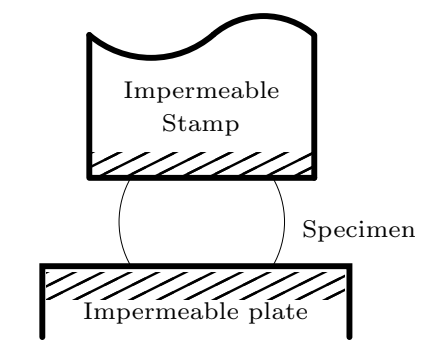

Unconfined compression test

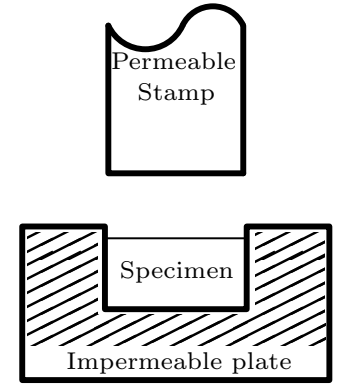

Confined compression test

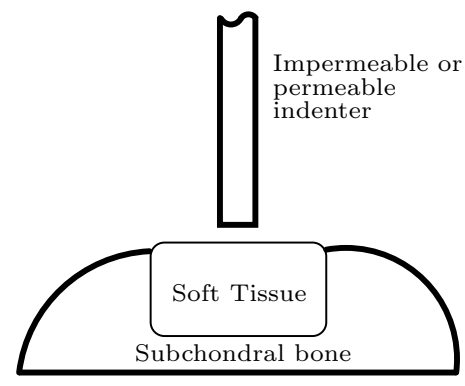

Indentation test

Figure 5.12: Various compression tests 
The most common compression tests of a viscoelastic material are stress relaxation tests and creep tests.

In stress-relaxation tests, a predefined compressive strain is applied and the corresponding stress is measured as a a function of time, left Fig. 5.13. With the relation between the constant increasing strain $(0-A)$ and the corresponding stress $\left(0-A^{\prime}\right)$, Young's modulus is obtained. If the strain is held constant $(A-B)$, the stress decreases with time $\left(A^{\prime}-B^{\prime}\right.$, stress relaxation).

In creep test, a predefined compressive stress is applied and the corresponding strain is measured as a a function of time, right Fig. 5.13. With the relation between the constant increasing stress $(0-C)$ and the corresponding strain $\left(0-C^{\prime}\right)$, Young's modulus is obtained. Holding the stress constant $(C-D)$, the strain increases with time $\left(C^{\prime}-D^{\prime}\right.$, creep).

Stress relaxation tests and creep tests in case of tension has the same principle as in the compression test. A specimen is stretched and the force is traction.

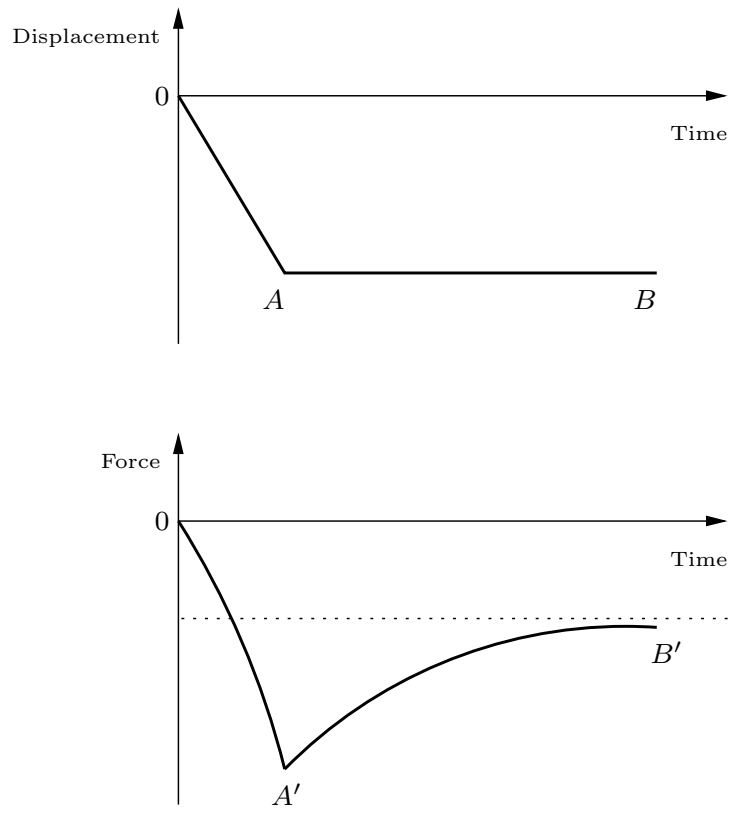

Relaxation
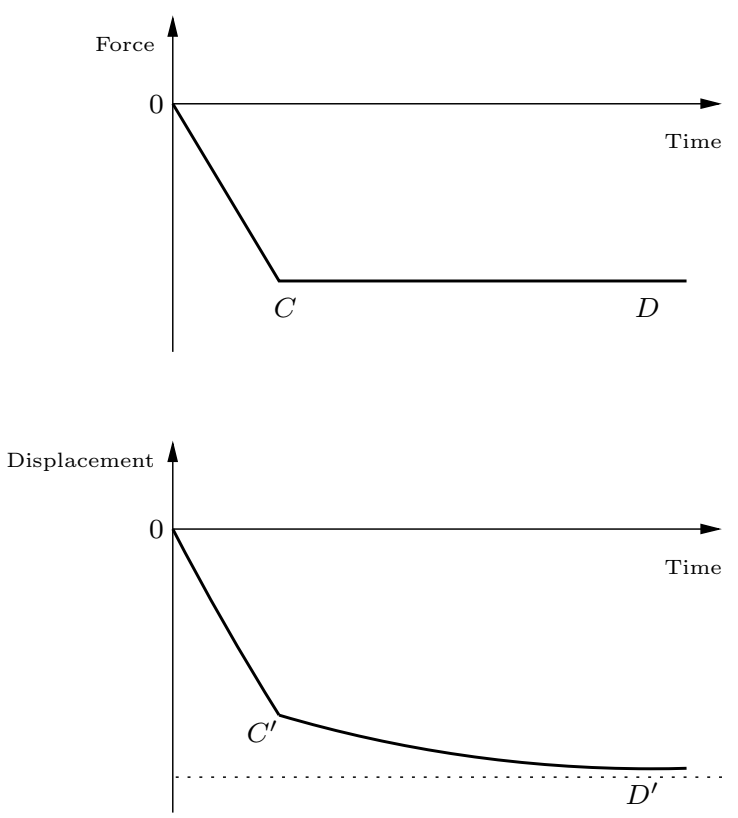

Creep

Figure 5.13: Relaxation and creep 


\subsection{Compression test}

\subsubsection{Compression testing}

As the given geometry of specimen, Section 5.1 (suitable only for compression test), a compression test is performed. Among them an unconfined compression test is carried out for material properties testing. Figure 5.14 shows a compression test on a MTS material testing machine.
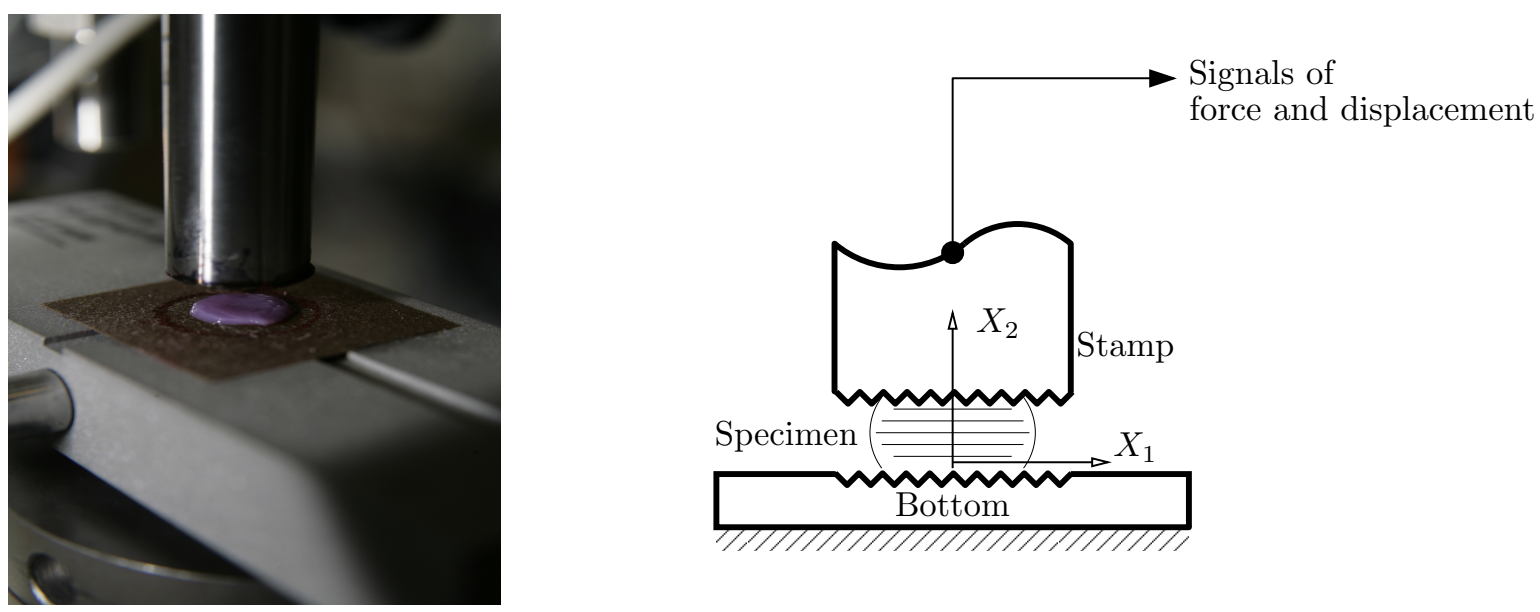

Figure 5.14: Compression test

The specimen is laid on a sandpaper and compressed with a stamp whose end is also covered with a sandpaper. Due to the sandpaper the specimen is compressed without sliding and the fluid of the specimen is squeezed only in directions perpendicular to $X_{2}$ (undrained in $X_{2}$-direction). In $X_{1}$ and $X_{3}$ directions the specimen is unconstrained.

In order to measure the Young's modulus, the specimen is compressed by a third of its thickness at a given velocity (compressional velocity $=0.5 \mathrm{~mm} / \mathrm{s}$ ). The reaction force and the displacement of stamp (displacement of specimen top edge) are recorded over time. Then the position of the stamp is kept constant at the height of two third of the thickness of the specimen for 20 seconds. After that, the specimen is released with the same velocity. 


\subsubsection{Signals from compression tests}

The diagrams in Fig. 5.15 are the signals of the compression tests. The green dashed line represents the displacement of specimen top edge and the red line represents the reaction force.
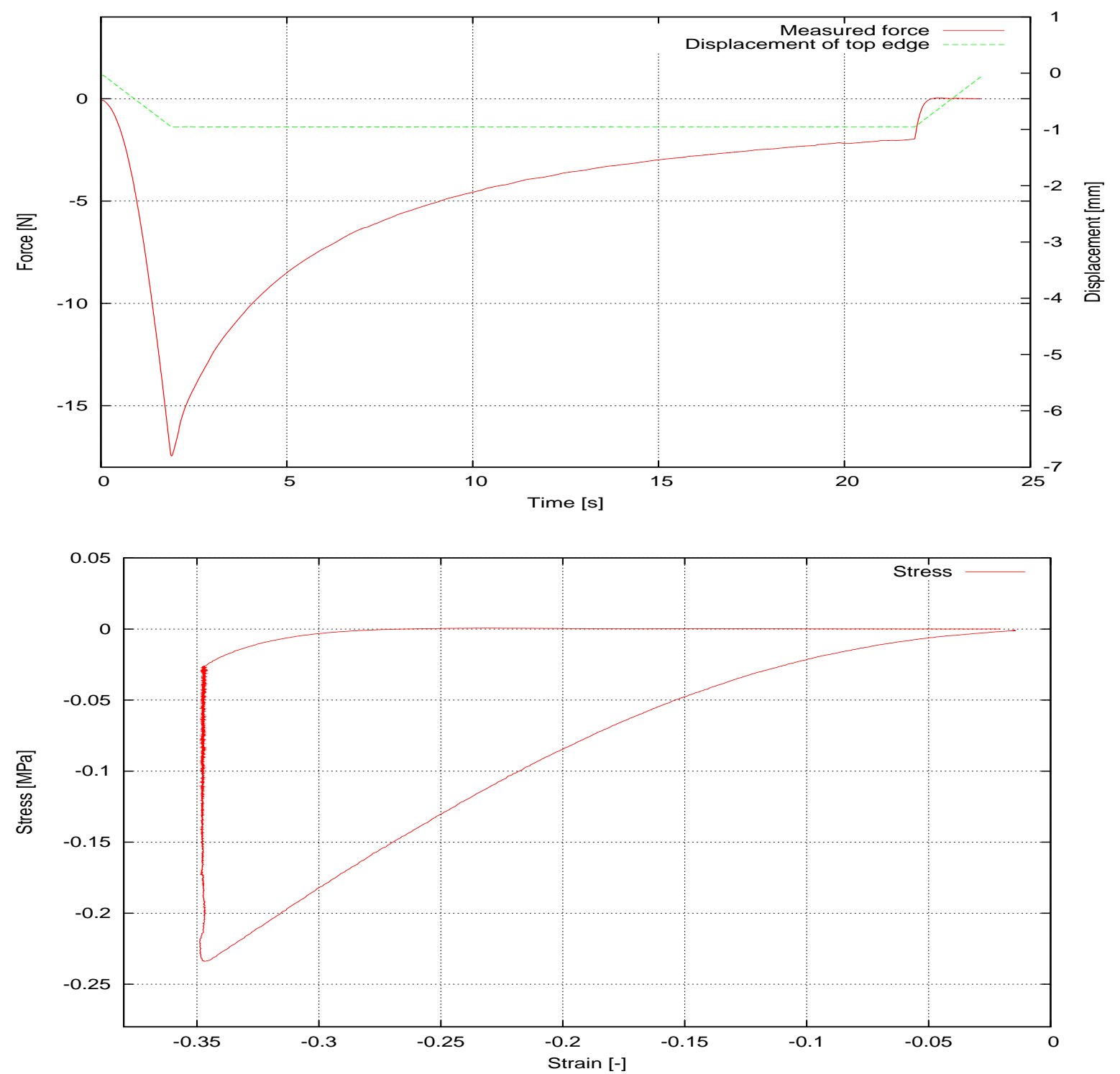

Figure 5.15: Signals from a compression test, before bioreactor stimulation

The curve is divided into three phases: compression at a constant strain rate, stress relaxation under a constant strain, and the release. For less than $5 \%$ strain, the material behavior is linear. Under a constant strain of about -0.35 , a decrease of the stress (stress relaxation from 
$-0.23 \mathrm{MPa}$ to $-0.0025 \mathrm{MPa}$ for 20 seconds) is observed.

The closed area is a hysteresis loop and shows the amount of energy lost in a loading and unloading cycle. It is equal to $\oint \sigma d \varepsilon$, where symbols are defined in the beginning.

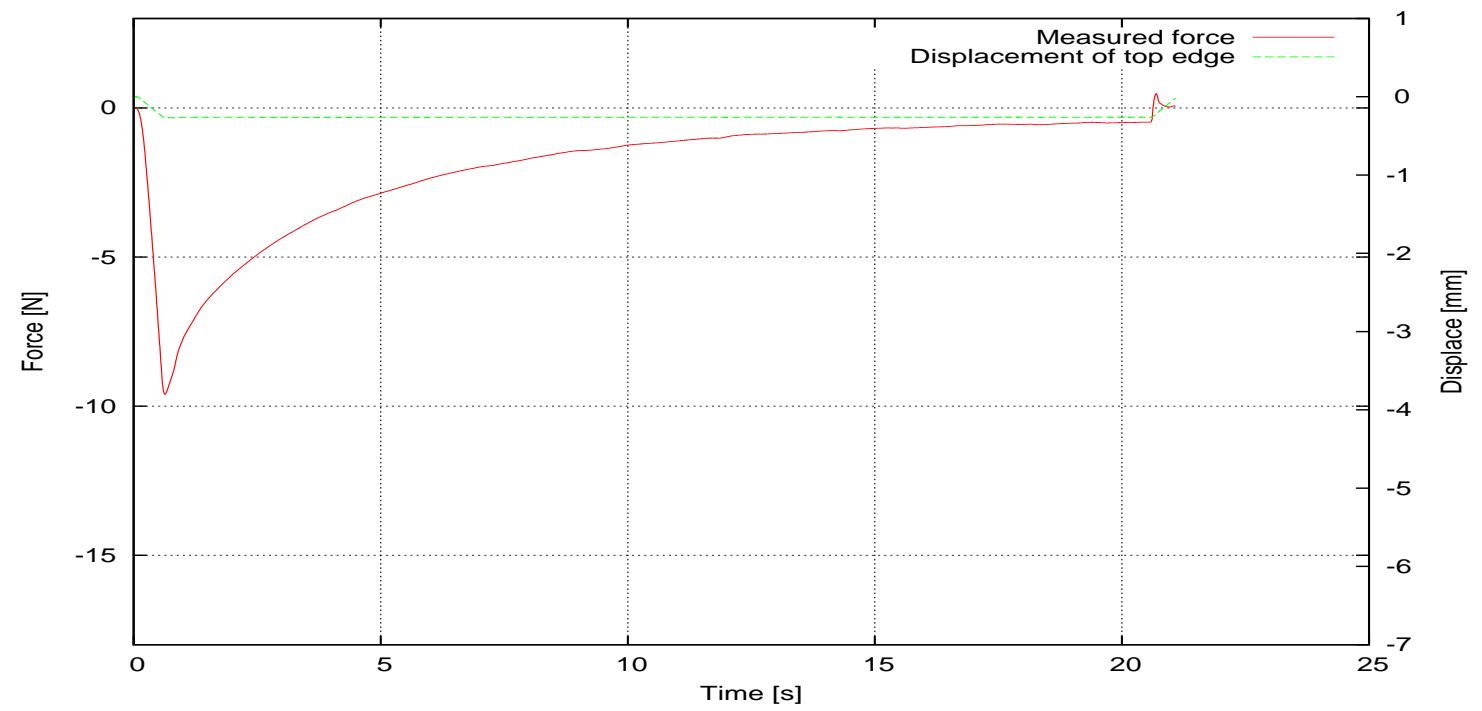

Figure 5.16: Signals from a compression test, after a one-week bioreactor stimulation

Due to the continuous periodic loading, the material is condensed (volumetric decrease), and the specimen becomes thinner. The condensing process is distinct during the first week.

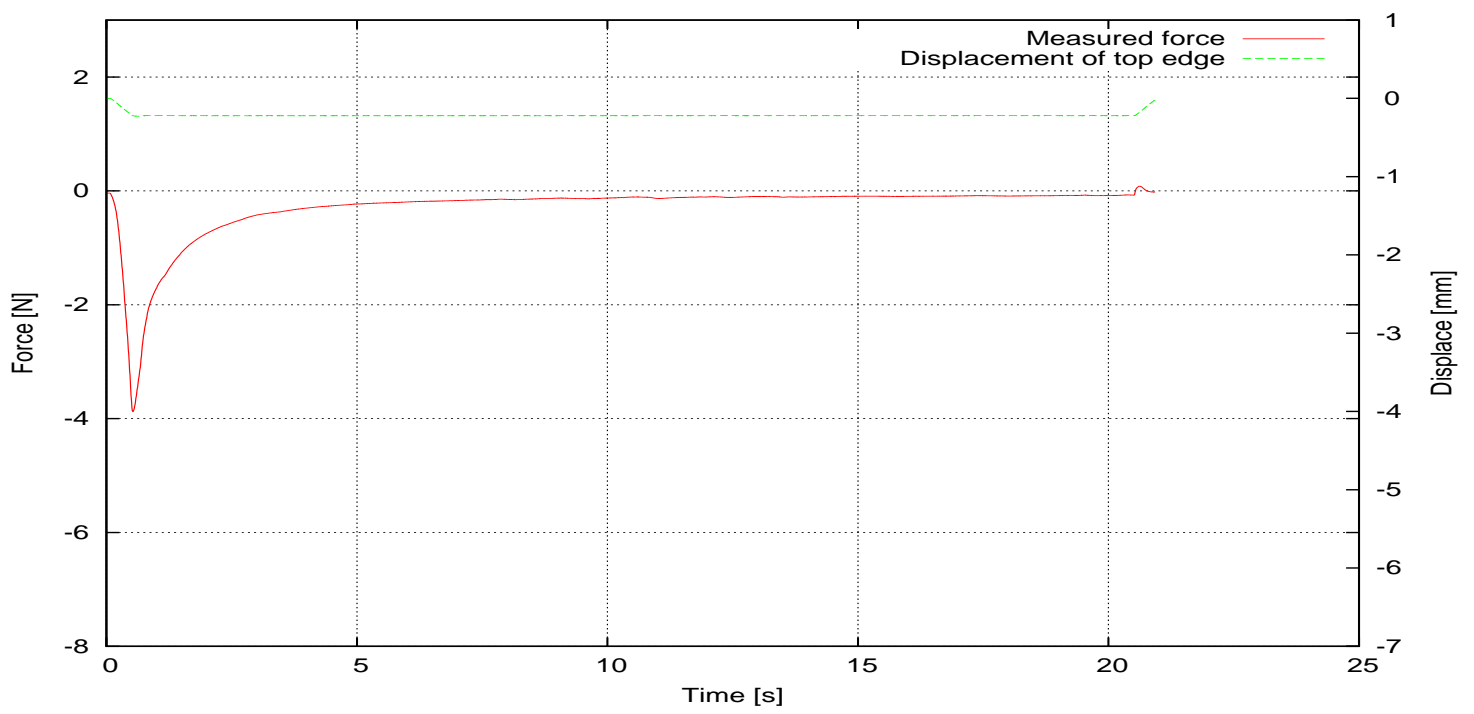

Figure 5.17: Signals from a compression test, after a two-week bioreactor stimulation 


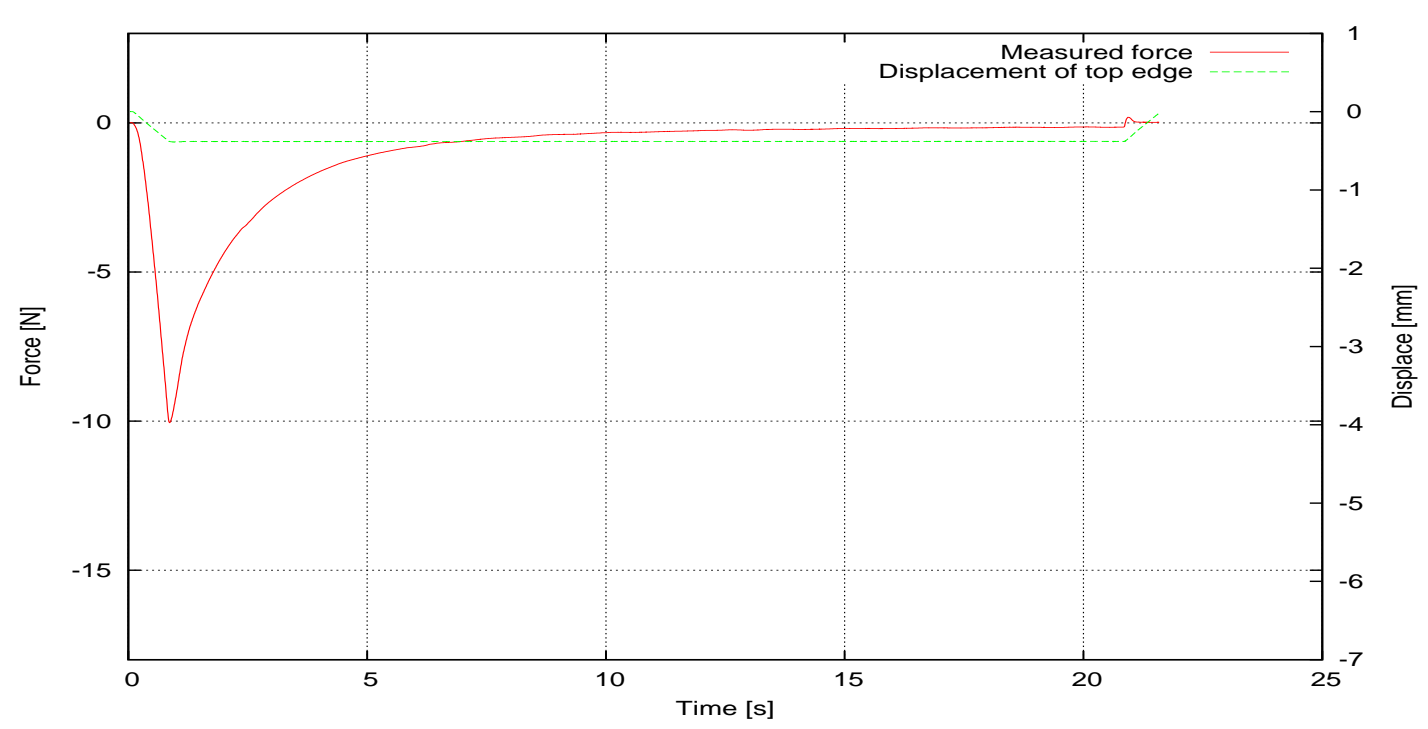

Figure 5.18: Signals from a compression test, after a third-week bioreactor stimulation

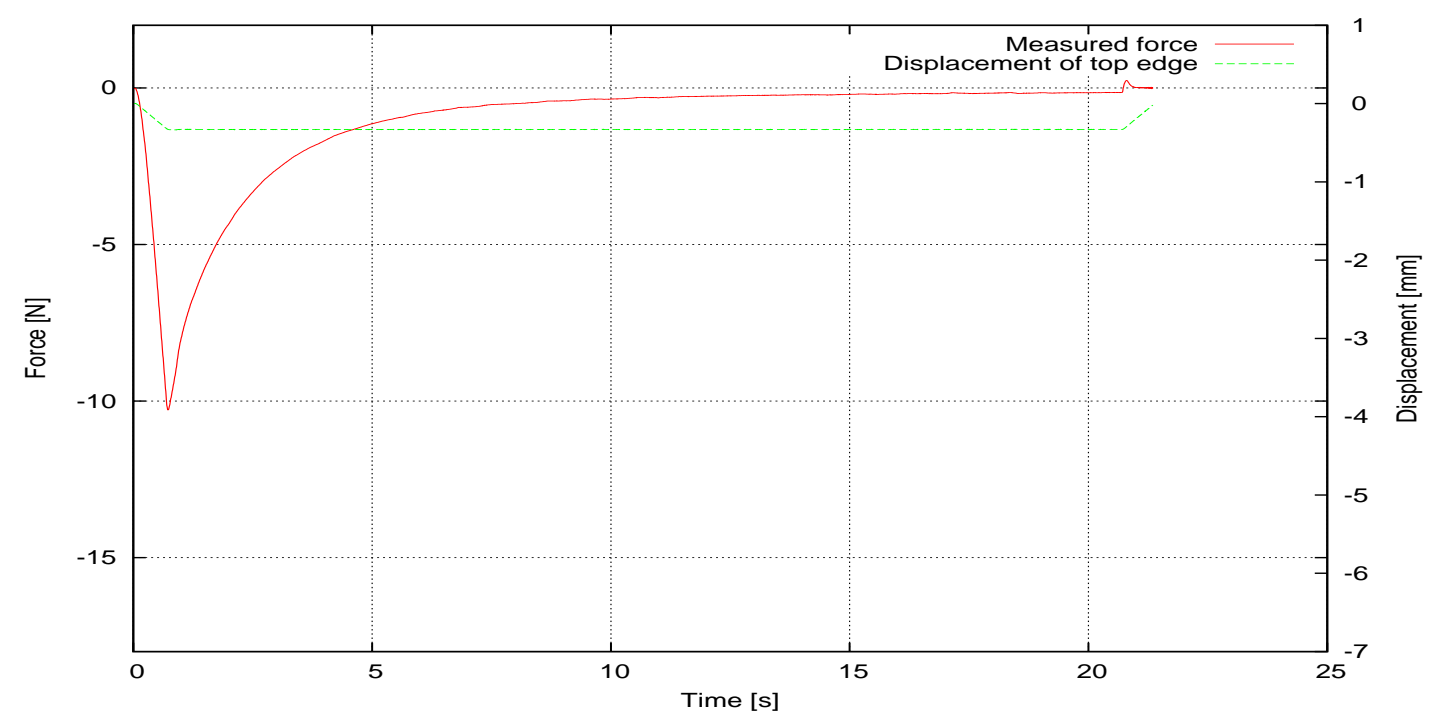

Figure 5.19: Signals from a compression test, after a four-week bioreactor stimulation

As seen in Fig. 5.15, the material behavior under compression is non-linear and the stressrelaxation due to fluid behavior is noticeable. In order to validate the proposed model, Section 4.3, we have to compare the simulation curve with the above compression test results.

Since thickness and diameter of specimen are different from each other due to various condensing process of material, the different geometry of specimen should be taken into account.

The thickness of specimen becomes thinner, as fluid flows out during the comression phase and 
does not flow in in the release phase. The positive force in small peaks of the release phase in Fig. 5.16, 5.17, 5.18 and 5.19 is a drag force due to adhesion and weight of specimen hanging at the stamp. 


\section{Chapter 6}

\section{Numerical modeling and results}

\subsection{Numerical model}

The stiffness matrix $\mathbb{C}^{\mathrm{e}}$ in Eq. (4.3.59) can be rewritten with Young's modulus $E_{i}$, shear modulus $G_{i j}$ and Poisson's ratios $\nu_{i j}(i \neq j)$, Vogler et al. [83]:

- Due to symmetry of the elasticity tensor, we have the following relations:

$$
\frac{\nu_{12}}{E_{1}}=\frac{\nu_{21}}{E_{2}} ; \quad \frac{\nu_{23}}{E_{2}}=\frac{\nu_{32}}{E_{3}} ; \quad \frac{\nu_{13}}{E_{1}}=\frac{\nu_{31}}{E_{3}}
$$

- Constants of invariant formulation can be replaced with the Young's modulus, shear modulus and Poisson's ratios:

$$
\begin{aligned}
\lambda & =E_{1}\left(\nu_{13}+\nu_{32} \nu_{23}\right) / Z \\
\alpha & =E_{1}\left[\nu_{32}\left(1+\nu_{31}-\nu_{23}\right)-\nu_{31}\right] / Z \\
\beta & \left.=E_{2}\left(1-\nu_{31} \nu_{13}\right) / Z-E_{1}\left(\nu_{13}+\nu_{23} \nu_{32}\right)\right] / Z-4 G_{21}, \\
G_{t} & =G_{21} \\
G_{p} & =G_{13} \\
Z & =1-\nu_{31}^{2}-2 \nu_{23} \nu_{32}-2 \nu_{31} \nu_{32} \nu_{23} .
\end{aligned}
$$


- Material parameters:

$E_{1}=E_{3} \stackrel{\text { def }}{=} E_{p}, \quad \nu_{13}=\nu_{31} \stackrel{\text { def }}{=} \nu_{p}, \quad \nu_{21}=\nu_{23} \stackrel{\text { def }}{=} \nu_{t p}, \quad \nu_{12}=\nu_{32} \stackrel{\text { def }}{=} \nu_{p t}$,

$E_{2} \stackrel{\text { def }}{=} E_{t}, \quad G_{21}=G_{23} \stackrel{\text { def }}{=} G_{t}, \quad G_{13}=G_{p}=E_{p} / 2\left(1+\nu_{p}\right) \quad$ on the isotropic plane

$E_{t}=-\left(\lambda G_{p}-4 \lambda G_{t}-\lambda \beta-2 \alpha G_{p}+2 G_{p}^{2}-\beta G_{p}-2 \alpha G_{p}-4 G_{t} G_{p}+\alpha^{2}\right) /\left(\lambda+G_{p}\right)$

$E_{p}=-4 G_{p}\left(\lambda G_{p}-4 \lambda G_{t}-\lambda \beta+2 G_{p}^{2}-\beta G_{p}-2 \alpha G_{p}-4 G_{t} G_{p}+\alpha^{2}\right) / Z_{t}$

$\nu_{t p}=2 G_{p}(\lambda+\alpha) / Z_{t}, \quad \nu_{p t}=(\lambda+\alpha) /\left(2 \lambda+2 G_{p}\right)$,

$\nu_{p}=-\left(\alpha^{2}+2 \lambda G_{p}-\beta \lambda-4 G_{t} \lambda\right) / Z_{t}$,

$D_{t}=4 G_{t} \lambda+\beta \lambda-4 G_{p}^{2}+4 G_{p} \alpha+2 \beta G_{p}+8 G_{t} G_{p}-\alpha^{2}$.

Then the stiffness matrix $\mathbb{C}^{e}$ can be calculated and the stress-strain law gives :

$\boldsymbol{\sigma}=\mathbb{C}^{\mathrm{e}} \boldsymbol{\epsilon}$

$$
\left\{\begin{array}{c}
\sigma_{11} \\
\sigma_{22} \\
\sigma_{33} \\
\sigma_{12} \\
\sigma_{13} \\
\sigma_{23}
\end{array}\right\}=\left[\begin{array}{cccccc}
\frac{1-\nu_{p t} \nu_{t p}}{E_{p} E_{t} \Gamma} & \frac{\nu_{t p}+\nu_{p} \nu_{t p}}{E_{p} E_{t} \Gamma} & \frac{\nu_{p}+\nu_{t p} \nu_{p t}}{E_{p} E_{t} \Gamma} & 0 & 0 & 0 \\
\frac{\nu_{p t}+\nu_{p} \nu_{p t}}{E_{p}^{2} \Gamma} & \frac{1-\nu_{p}^{2}}{E_{p}^{2} \Gamma} & \frac{\nu_{p t}+\nu_{p t} \nu_{p}}{E_{p}^{2} \Gamma} & 0 & 0 & 0 \\
\frac{\nu_{p}+\nu_{p t} \nu_{t p}}{E_{t} E_{p} \Gamma} & \frac{\nu_{t p}+\nu_{t p} \nu_{p}}{E_{t} E_{p} \Gamma} & \frac{1-\nu_{t p} \nu_{p t}}{E_{t} E_{p} \Gamma} & 0 & 0 & 0 \\
0 & 0 & 0 & G_{t} & 0 & 0 \\
0 & 0 & 0 & 0 & G_{p} & 0 \\
0 & 0 & 0 & 0 & 0 & G_{t}
\end{array}\right]\left\{\begin{array}{c}
\varepsilon_{11} \\
\varepsilon_{22} \\
\varepsilon_{33} \\
\gamma_{12} \\
\gamma_{13}
\end{array}\right\}
$$

where $\Gamma=\frac{\left(1+\nu_{p}\right)\left(1-\nu_{p}-2 \nu_{p t} \nu_{t p}\right)}{E_{p}^{2} E_{t}}$.

Using only five material constants $E_{p}, E_{t}, \nu_{p}, \nu_{p t}$ and $G_{t}$, the above equation (6.1.3) can be 

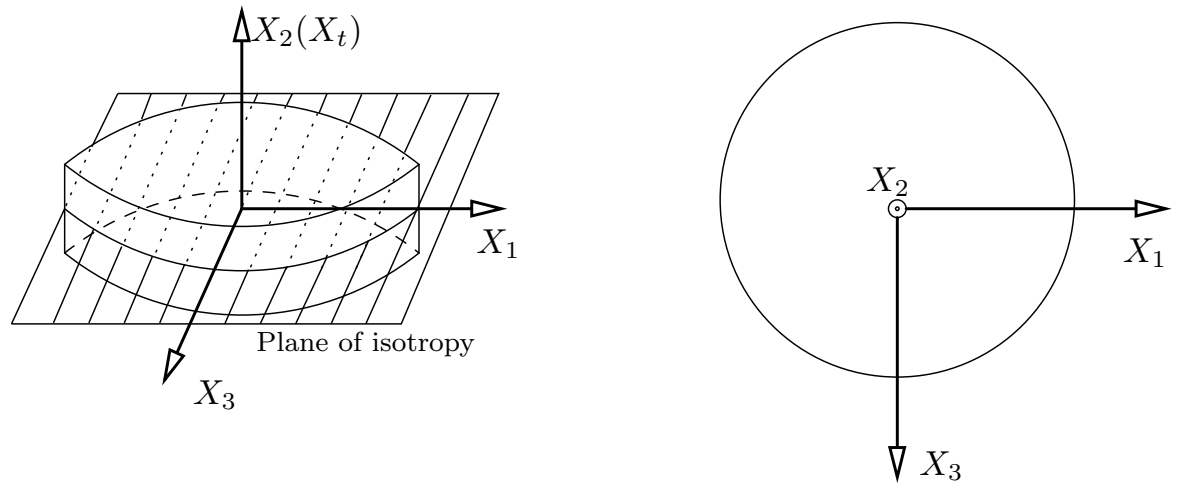

Figure 6.1: Coordinate of the cylindrical specimen

rewritten:

$\left\{\begin{array}{c}\sigma_{11} \\ \sigma_{22} \\ \sigma_{33} \\ \sigma_{12} \\ \sigma_{13} \\ \sigma_{23}\end{array}\right\}=\left[\begin{array}{cccccc}\frac{E_{p}-\nu_{p t}^{2} E_{t}}{E_{p}^{2} E_{t} \Gamma} & \frac{\nu_{p t}\left(1+\nu_{p}\right)}{E_{p}^{2} \Gamma} & \frac{\nu_{p} E_{p}+\nu_{p t}^{2} E_{t}}{E_{p}^{2} E_{t} \Gamma} & 0 & 0 & 0 \\ \frac{\nu_{p t}\left(1+\nu_{p}\right)}{E_{p}^{2} \Gamma} & \frac{1-\nu_{p}^{2}}{E_{p}^{2} \Gamma} & \frac{\nu_{p t}\left(1+\nu_{p}\right)}{E_{p}^{2} \Gamma} & 0 & 0 & 0 \\ \frac{\nu_{p} E_{p}+\nu_{p t}^{2} E_{t}}{E_{p}^{2} E_{t} \Gamma} & \frac{\nu_{p t}\left(1+\nu_{p}\right)}{E_{p}^{2} \Gamma} & \frac{E_{p}-\nu_{p t}^{2} E_{t}}{E_{p}^{2} E_{t} \Gamma} & 0 & 0 & 0 \\ 0 & 0 & 0 & G_{t} & 0 & 0 \\ 0 & 0 & 0 & 0 & G_{p} & 0 \\ 0 & 0 & 0 & 0 & 0 & G_{t}\end{array}\right]\left\{\begin{array}{c}\varepsilon_{11} \\ \varepsilon_{22} \\ \varepsilon_{33} \\ \gamma_{12} \\ \gamma_{13} \\ \gamma_{23}\end{array}\right\}$

where $\Gamma=\frac{\left(1+\nu_{p}\right)\left(E_{p}-E_{p} \nu_{p}-2 \nu_{p t}^{2} E_{t}\right)}{E_{p}^{3} E_{t}}$.

The specimens used in our experiments are rotationally symmetric, Fig. 6.1. In the unconfined compression test whose loading direction is $X_{2}$ the strains have the following relations due to the transversely isotropy:

$$
\varepsilon_{11}=\varepsilon_{33} \quad \text { and } \quad \gamma_{12}=\gamma_{23}
$$

The normal stresses in $X_{1}-$ and $X_{3}$ - directions are the same:

$$
\sigma_{11}=\sigma_{33}=\frac{E_{p}+\nu_{p} E_{p}-2 \nu_{p t}^{2} E_{t}}{E_{p}^{2} E_{t} \Gamma} \varepsilon_{11}+\frac{\nu_{p t}\left(1+\nu_{p}\right)}{E_{p}^{2} \Gamma} \varepsilon_{22} .
$$

The normal stress in $X_{2}-$ direction

$$
\sigma_{22}=\frac{2 \nu_{p t}\left(1+\nu_{p}\right)}{E_{p}^{2} \Gamma} \varepsilon_{11}+\frac{1-\nu_{p}^{2}}{E_{p}^{2} \Gamma} \varepsilon_{22} .
$$


The shear stresses are

$\sigma_{12}=\sigma_{23}=G_{t} \gamma_{12} \quad$ and $\quad \sigma_{13}=G_{p} \gamma_{13}$

$$
\left\{\begin{array}{c}
\sigma_{11} \\
\sigma_{22} \\
\sigma_{12} \\
\sigma_{13}
\end{array}\right\}=\left[\begin{array}{cccc}
\frac{E_{p}+\nu_{p} E_{p}-2 \nu_{p t}^{2} E_{t}}{E_{p}^{2} E_{t} \Gamma} & \frac{\nu_{p t}\left(1+\nu_{p}\right)}{E_{p}^{2} \Gamma} & 0 & 0 \\
\frac{2 \nu_{p t}\left(1+\nu_{p}\right)}{E_{p}^{2} \Gamma} & \frac{1-\nu_{p}^{2}}{E_{p}^{2} \Gamma} & 0 & 0 \\
0 & 0 & G_{t} & 0 \\
0 & 0 & 0 & \frac{E_{p}}{2\left(1+\nu_{p}\right)}
\end{array}\right]\left\{\begin{array}{c}
\varepsilon_{11} \\
\varepsilon_{22} \\
\gamma_{12} \\
\gamma_{13}
\end{array}\right\} .
$$

Here the stiffness component $C_{1122}$ and $C_{2211}$ are not the same due to boundary conditions (axial symmetry about the axis $X_{2}$ ).

Due to the third simplification in section 4.3.3, the initial material is assumed to be isotropic:

$$
\begin{aligned}
& E_{t}=E_{p} \stackrel{\text { def }}{=} E, \quad \nu_{p}=\nu_{p t}=\nu, \quad G_{t} \stackrel{\text { def }}{=} G=\frac{E}{2(1+\nu)} . \\
& \left\{\begin{array}{c}
\sigma_{11} \\
\sigma_{22} \\
\sigma_{12} \\
\sigma_{13}
\end{array}\right\}=\left[\begin{array}{cccc}
\frac{E\left(1+\nu-2 \nu^{2}\right)}{(1+\nu)\left(1-\nu-2 \nu^{2}\right)} & \frac{E \nu}{1-\nu-2 \nu^{2}} & 0 & 0 \\
\frac{2 E \nu}{1-\nu-2 \nu^{2}} & \frac{E(1-\nu)}{(1+\nu)(1-2 \nu)} & 0 & 0 \\
0 & 0 & \frac{E}{2(1+\nu)} & 0 \\
0 & 0 & 0 & \frac{E}{2(1+\nu)}
\end{array}\right]\left\{\begin{array}{c}
\varepsilon_{11} \\
\gamma_{13}
\end{array}\right\} .
\end{aligned}
$$

As explained in Section 4.3.4, the stress evolves in two different scales: a short time-scale evolution, Subsection 6.1.1, and the long time-scale evolution, Subsection 6.1.2. 


\subsubsection{Short time-scale evolution}

Now we fit the constitutive model to the experimental data obtained from compression tests, Subsection 5.2.3, in order to identify the material parameters. They can be identified by performing an iterative optimization process, Zhou et al. [91].

The incremental form of the total stress (4.3.76) is

$$
\begin{aligned}
\Delta \boldsymbol{\sigma}_{t+\Delta t} & =\Delta \boldsymbol{\sigma}_{t+\Delta t}^{\mathrm{e}}+\Delta \boldsymbol{\sigma}_{t+\Delta t}^{\mathrm{d}} \\
& =\mathbb{C}^{\mathrm{e}}: \Delta \boldsymbol{\epsilon}_{t+\Delta t}-D \operatorname{sph} \boldsymbol{\sigma}_{t+\Delta t} \Delta t
\end{aligned}
$$

Here $\Delta t$ denotes the time increment and $\mathbb{C}^{\mathrm{e}}$ is the stiffness matrix whose only Young's modulus $E_{p}$ is remodeled in long time-scale according to (6.1.15) and (6.1.16) .

The increment of the diffusion parameter (4.3.73) is

$$
\Delta D=D_{1} \Delta \varepsilon_{\mathrm{V}}
$$

This diffusive material model is implemented in user subroutine UMAT in ABAQUS:

$$
\mathbb{C}^{\mathrm{e}, t+\Delta t}=\frac{\partial \Delta \boldsymbol{\sigma}}{\partial \Delta \boldsymbol{\epsilon}}=\hat{\mathbb{C}}^{\mathrm{e}}(D)
$$

where $\Delta t$ is time increment in short time-scale.

\subsubsection{Long time-scale evolution}

For the long time-scale evolution the following assumptions are made:

1. Only Young's modulus on the isotropic plane $E_{p}$ changes. The other four material parameters $\left(E_{t}, \nu_{p}, \nu_{p t}\right.$ and $\left.G_{t}\right)$ are assumed not to be affected by the remodeling process.

2. $\nu_{p}=\nu_{p t} \stackrel{\text { def }}{=} \nu$.

3. $G_{t} \stackrel{\text { def }}{=} \frac{E}{2(1+\nu)}$.

where $\nu$ and $E$ are Poisson's ratio and Young's modulus of the initial isotropic state.

The first assumption is based on the phenomenon that the collagen type II fibers are synthesized 
on the isotropic plane (perpendicular to the loading direction $X_{2}$ ).

The following evolution equation is proposed for the Young's modulus $E_{p}$ according to (4.3.75):

$$
\begin{aligned}
& \dot{E}_{p}=k \sqrt[n]{\Psi}\left(E_{p, \text { crit }}-E_{p}\right) \\
& \Psi=\frac{1}{2} \lambda \ln ^{2}\left(\sqrt{I_{3}}\right)+\frac{1}{2} \mu\left(I_{1}-3\right)-\mu \ln \left(\sqrt{I_{3}}\right)
\end{aligned}
$$

where $E_{p, \text { crit }}$ is the critical Young's modulus that the material can reach with the maximum collagen type-II synthesization and is determined by compression test after four-week bioreactor stimulation. $I_{1}$ and $I_{3}$ are the first and the third invariant of the right Cauchy-Green strain tensor $\mathbf{C}, \Psi$ is a free energy density, $\lambda$ and $\mu$ are Lamé constants on the isotropic plane:

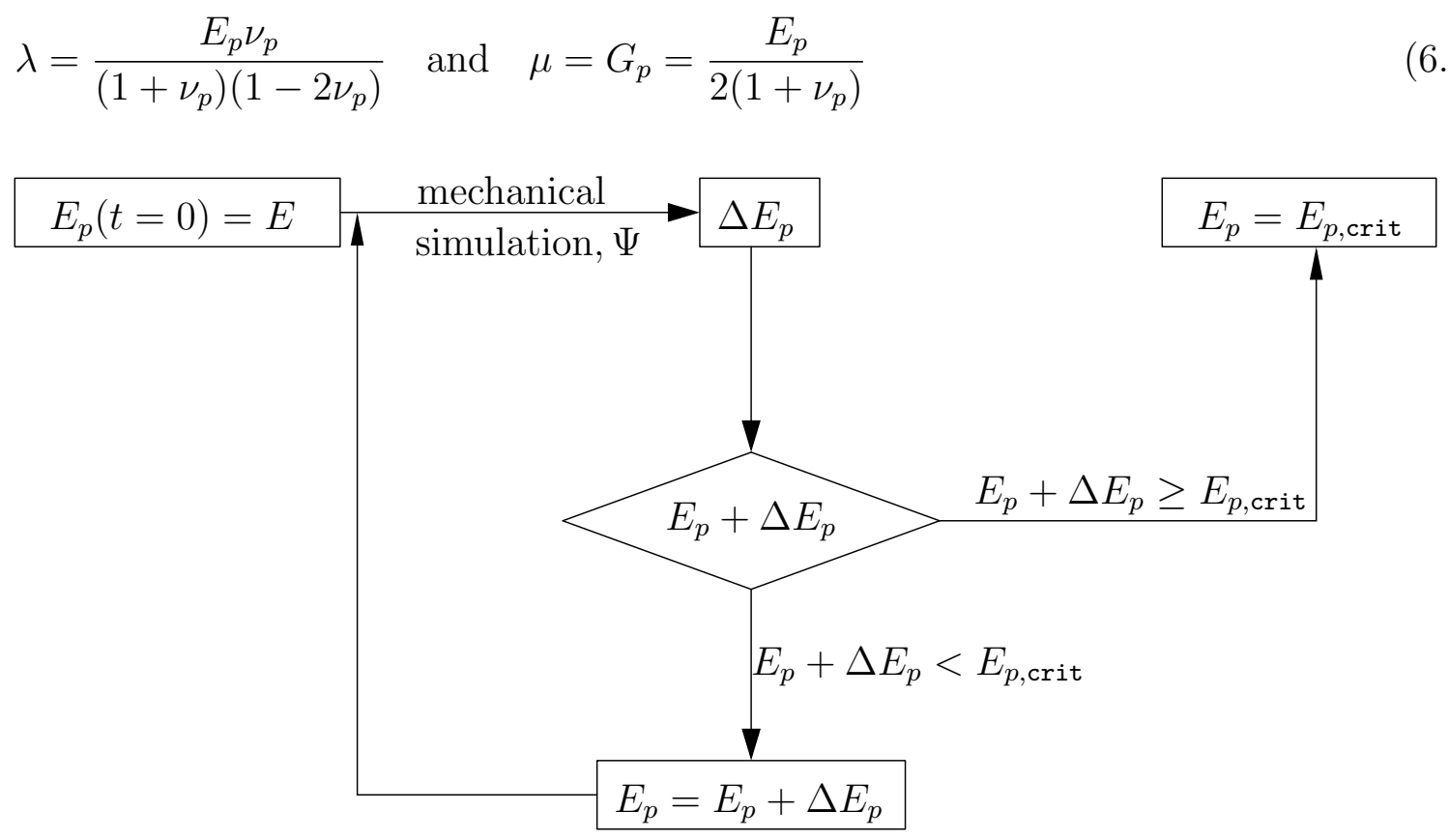

Figure 6.2: Flowchart of Young's modulus evolution

Due to the third assumption in section 4.3.3, the initial value of $E_{p}$ is $E$ and $E_{t}$ has the constant initial value $E$ through the bioreactor stimulation.

$\Delta E_{p}$ is the increment of the Young's modulus that results from the newly synthesized collagen type II. The synthesization is promoted through the mechanical stimulation $\Psi$ in a bioreactor. After time integration, $E_{p}$ becomes

$$
\Delta E_{p}^{t+\Delta t}=k \sqrt[n]{\Psi^{t+\Delta t}}\left(E_{p, \text { crit }}-E_{p}^{t}\right) \Delta t
$$


where $E_{p}^{t+\Delta t}$ and $\Psi^{t+\Delta t}$ are the current Young's modulus and the current free energy density, $E_{p}^{t}$ is the Young's modulus from the previous increment.

The above proposed material model is implemented in user subroutine UMAT in ABAQUS:

$$
\mathbb{C}^{\mathrm{e}, t+\Delta t}=\frac{\partial \Delta \boldsymbol{\sigma}}{\partial \Delta \boldsymbol{\epsilon}}=\hat{\mathbb{C}}^{\mathrm{e}}\left(\Psi^{t+\Delta t}\right)
$$

where $\Delta t$ is time increment in long time-scale. 


\subsection{Numerical calculations}

\subsubsection{Parameter identification with compression test}

This step is to fit the model suggested in (4.3.76) and (6.1.12) to experimental data from Subsection 5.4.2.

Due to the nonlinear elasticity of the material, the tangential modulus of a stress-strain curve is very various according to region, Fig. 5.15, and cannot be determined by reading out the curve. In order to identify the elastic modulus and diffusion parameters, we employed the finite element simulation. Zhou et al. [92] developed an optimization algorithm based on the method of steepest descent in order to identify elastic modulus and diffusion parameters.

Due to axisymmetric geometry subjected to axial loading, the element type is assigned CAX4R (A 4-node bilinear axisymmetric quadratic continuum element without twist), Fig. 6.3. The red lines in the following figures are the forces measured in compression tests and the green ones are forces calculated with ABAQUS. By solving an inverse problem, the corresponding elastic modulus and diffusion parameters can be determined.

From the compression test and the curve fitting of the non-stimulated specimen in initial state, the elastic modulus, $E_{p}(t=0)=E$ and the diffusion parameters, $D_{0}, D_{1}$, at initial state can be obtained, Fig. 6.4 .

The following curves, Fig. 6.5, Fig. 6.6, Fig. 6.7 and Fig. 6.8 show how $E_{p}(t \neq 0), D_{0}$ and $D_{1}$ change every week. The Table 6.1 is the summary of identified parameters. 


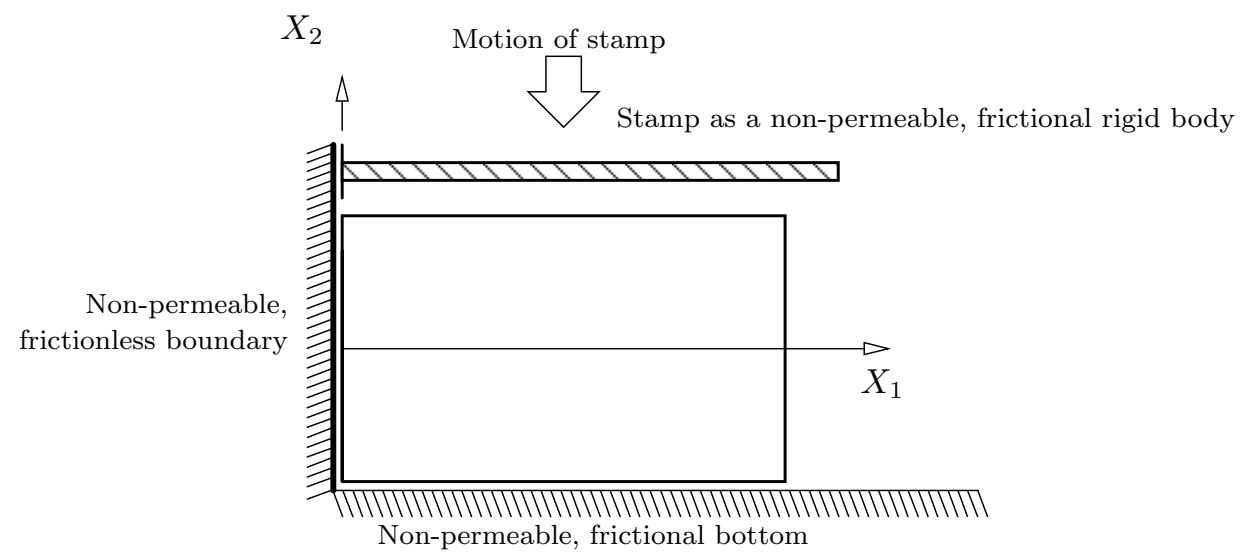

Figure 6.3: Cross section of the right side of a rotationally symmetric specimen

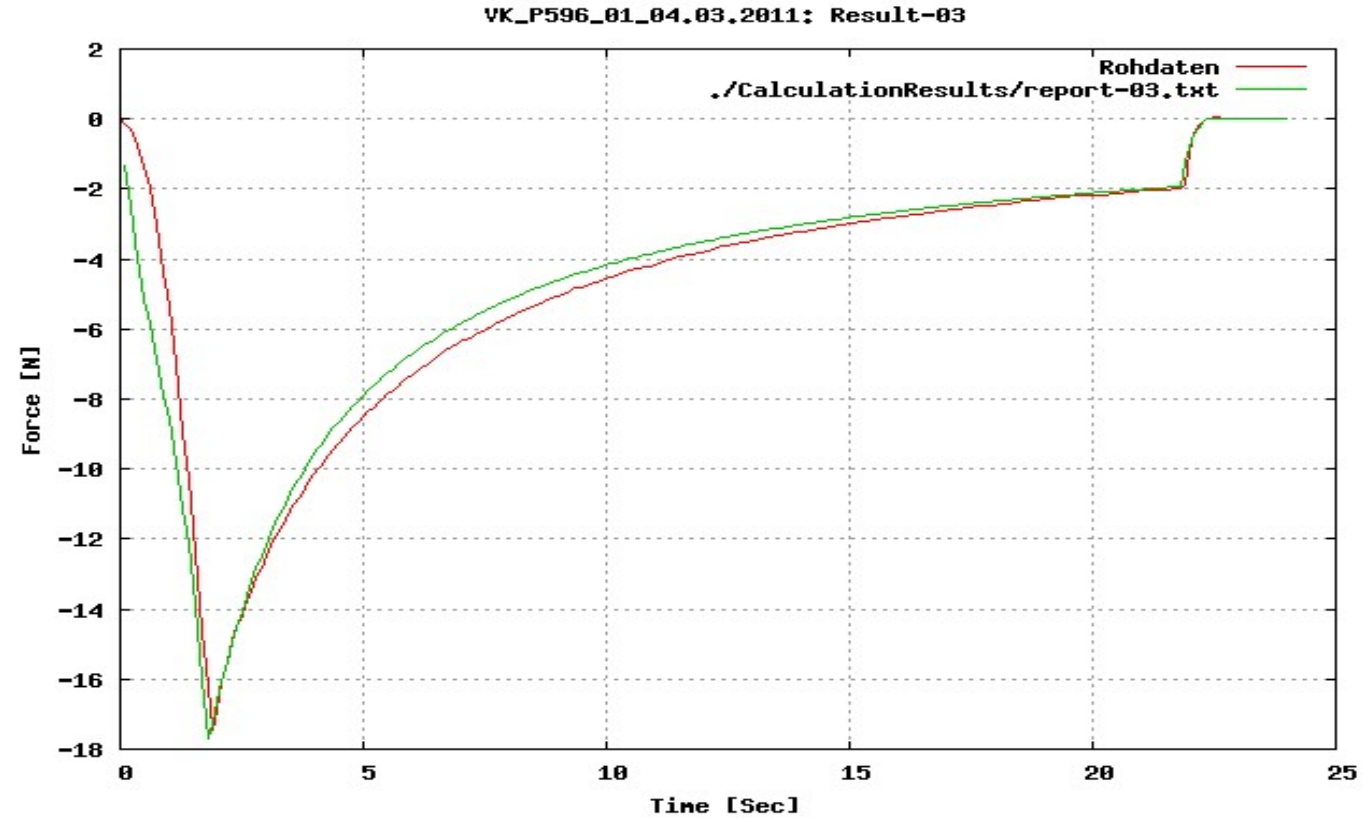

Figure 6.4: Before bioreactor stimulation (initial state) 


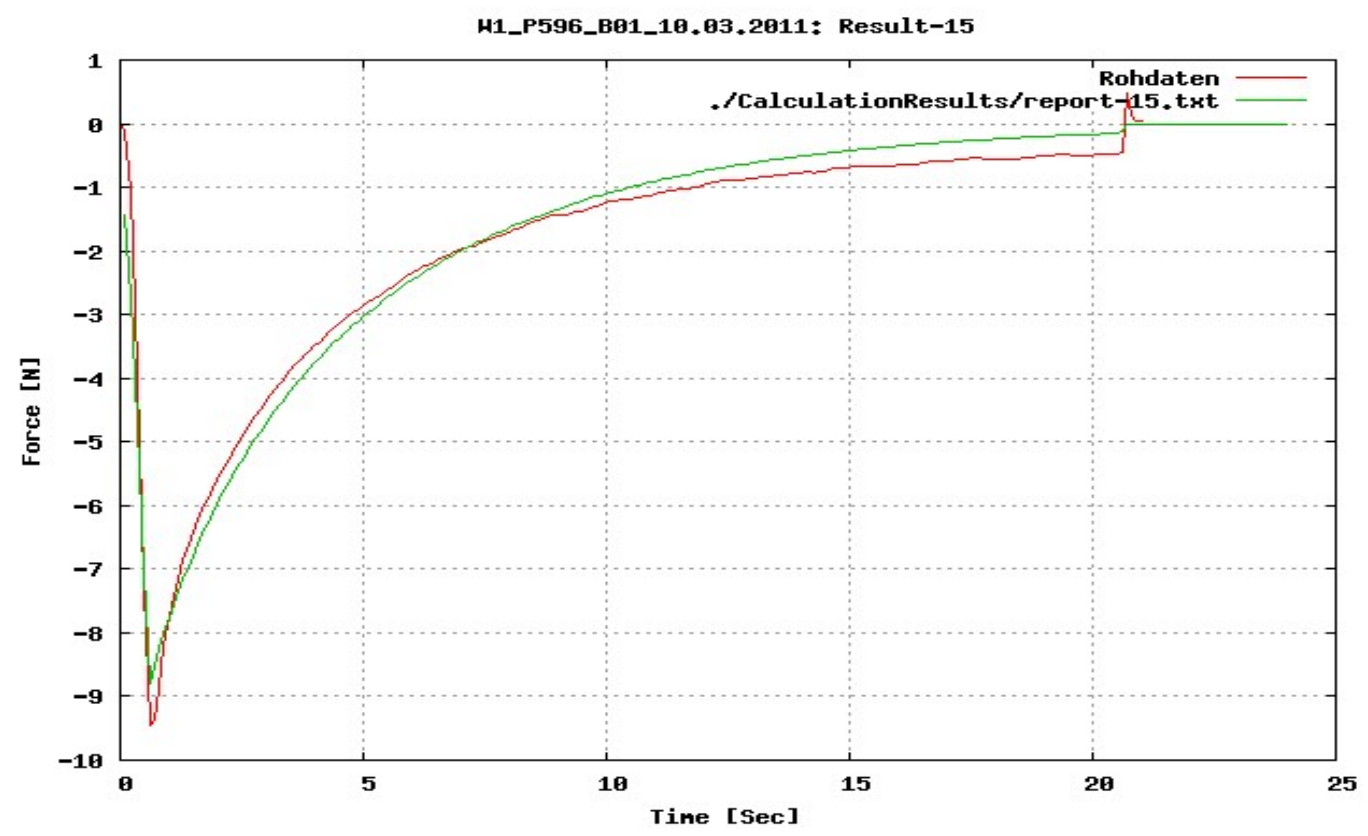

Figure 6.5: After 1-week Bioreactor stimulation

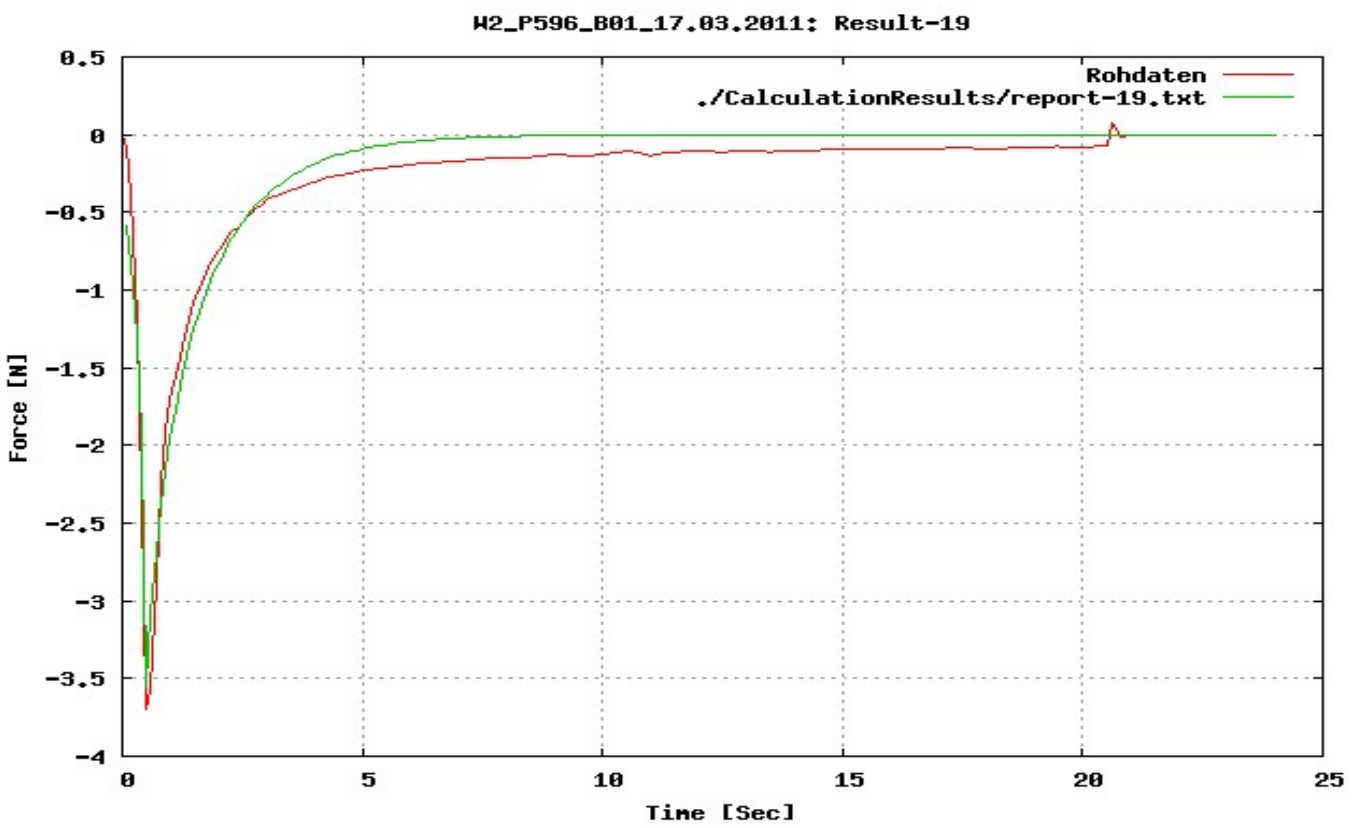

Figure 6.6: After 2-week Bioreactor stimulation 


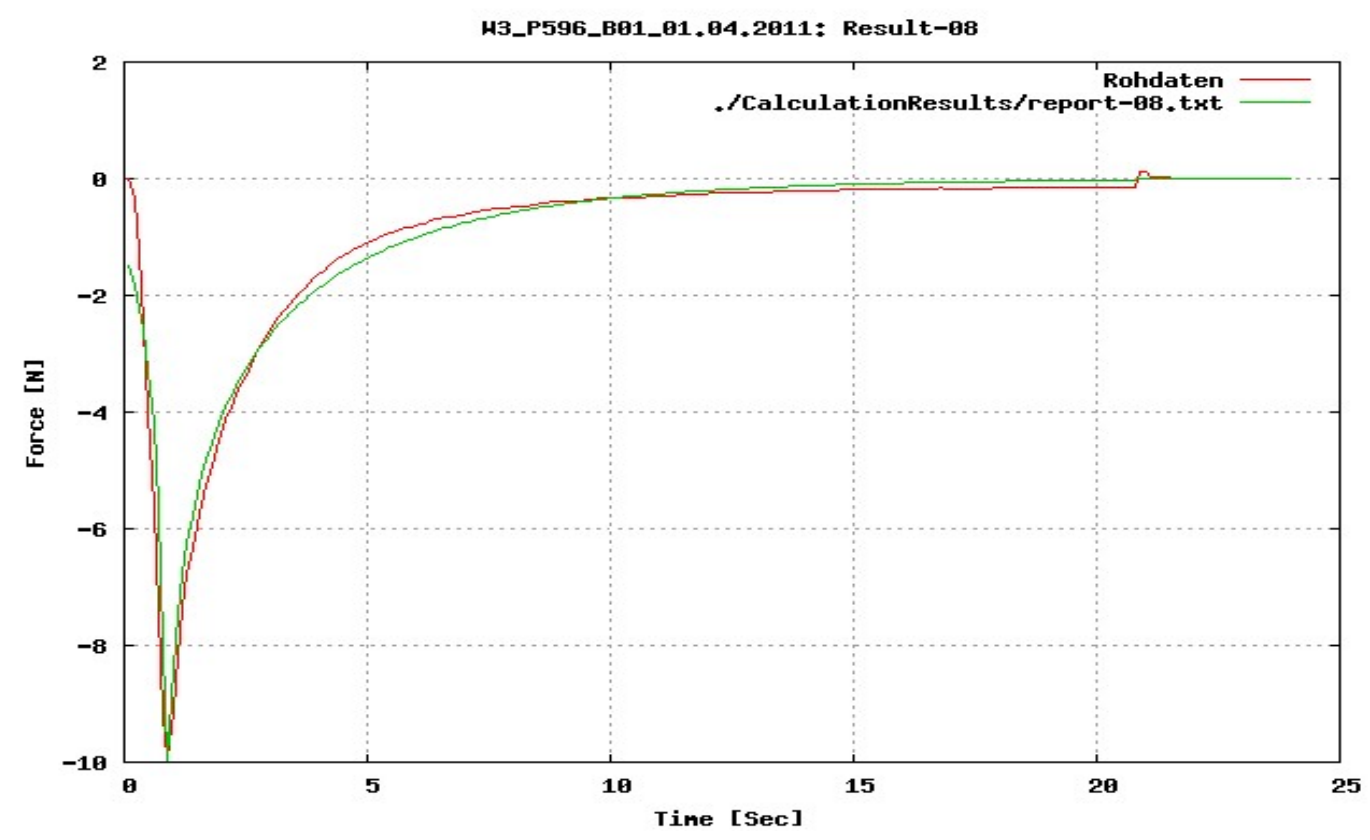

Figure 6.7: After 3-week Bioreactor stimulation

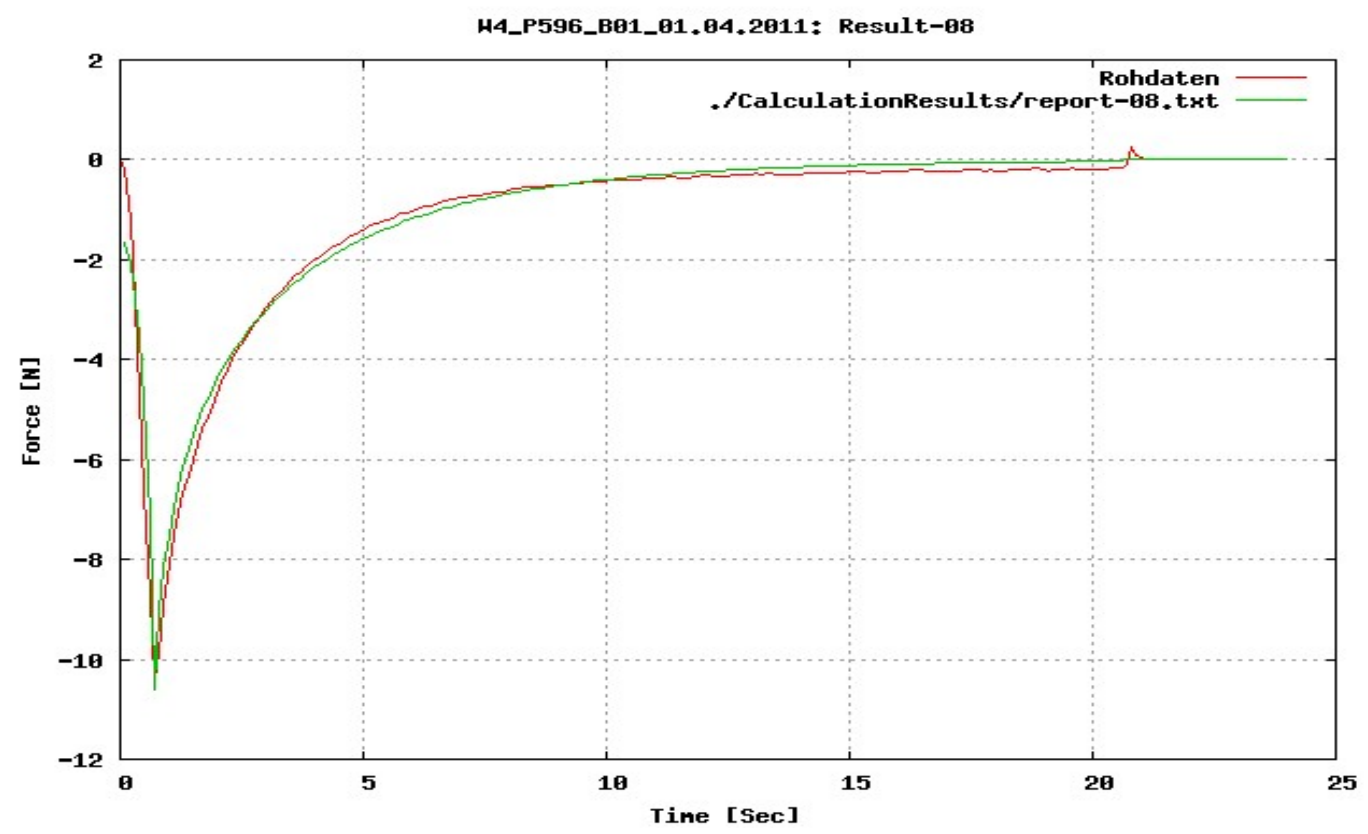

Figure 6.8: After 4-week Bioreactor stimulation 


\begin{tabular}{c|ccc}
\hline Time [week] & $E_{p}[\mathrm{MPa}]$ & $D_{0}\left[s^{-1}\right]$ & $D_{1}\left[s^{-1}\right]$ \\
\hline \hline Initial state & 0.8963 & 2.48 & 2.21 \\
After 1-week stimulation & 0.2145 & 2.81 & 2.17 \\
After 2-week stimulation & 0.5671 & 37.67 & 31.27 \\
After 3-week stimulation & 0.9866 & 28.03 & 23.01 \\
After 4-week stimulation & 0.9980 & 27.76 & 23.28 \\
\hline
\end{tabular}

Table 6.1: Modifications of elastic modulus and Diffusion parameters in a bioreactor

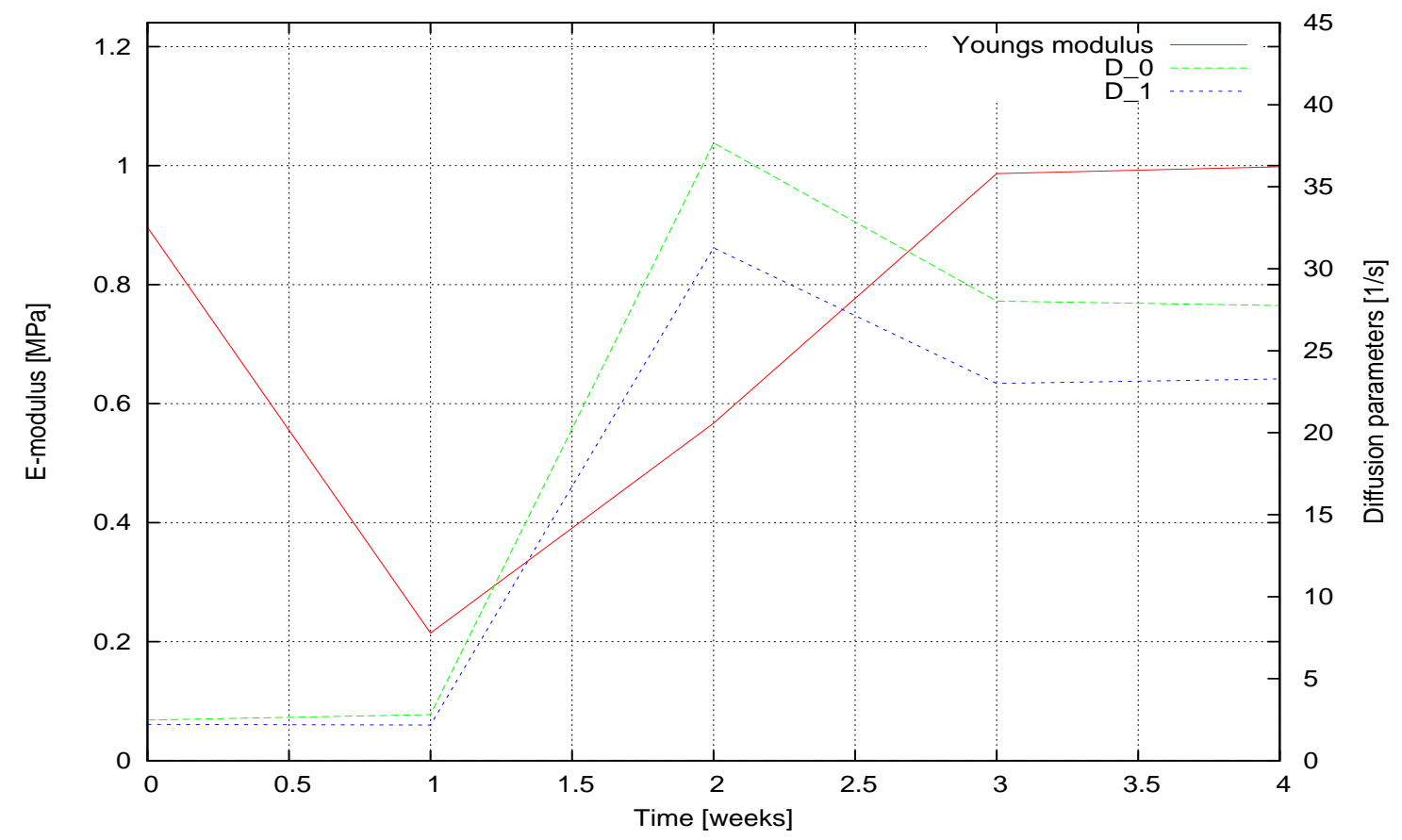

Figure 6.9: Modification of E-modulus, D0 and D1 in bioreactor 


\subsubsection{Remodeling curve}

The subject of the present work is to understand how material properties change due to the mechanical stimulation in a bioreactor. Fig. 6.9 shows the modifications of elastic modulus $E_{p}$ and Diffusion parameters $D_{0}, D_{1}$ over time, long time-scale evolution, [87] and [88].

Above all, elastic modulus is the main interest of the present simulation, as it shows stiffness of replacement materials. The elastic modulus decreases after the 1-week stimulation and increases obviously in the second and the third week. In the last week the increase becomes smaller. The increase is caused by synthesis of collagen type II fibers, as expected. On the contrary, the stiffness decreases in the first week and it is supposed that the cells donated by a patient are predamaged and the material degrades (an opposite of synthesis): This effect is called enzyme degradation and is assumed to occur in the first week of bioreactor stimulation. Enzymes are large biological molecules that are responsible for the chemical interconversions that sustain life. They control the synthesis or degradation of materials.

The slow curve of the last week shows that the synthesis of collagen type II is mainly reached about at third week.

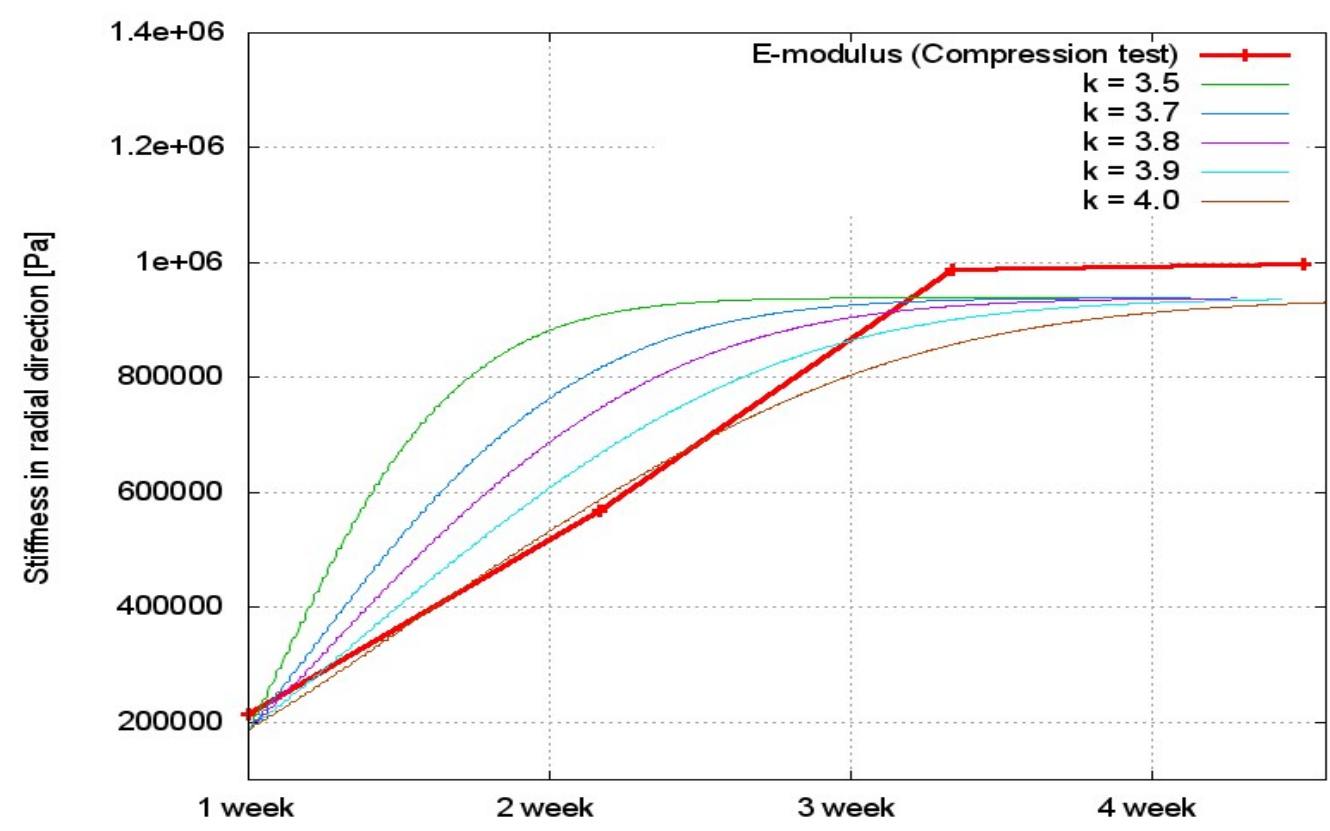

Figure 6.10: Remodeling curve of elastic modulus $E_{p}$ 
With (6.1.15), we can predict the remodeling curve of $E_{p}$. In the model only the case of synthesis is considered. The degradation case is regarded as unusual and excluded. By solving the inverse problem and fitting curve with various $k=3.5 \sim 4.3, n=0.4 \sim 0.6$, we obtain the remodeling curve with a combination of $k=4.0$ and $n=0.5$.

Figure 6.11 shows the right side of the model's cross section.

The distribution of elastic modulus $E_{p}$ are modified over time. In the first week the material is isotropic $\left(E_{p}=E\right)$ as simplification 3 in Section 4.3 .3 and as time goes by the material becomes transversely isotropic $\left(E_{p}>E\right)$ due to the newly synthesized collagen type II fibers. On the other hand, the other elastic modulus $E_{t}$ is assumed to be constant: $E_{t}=E$ over time.
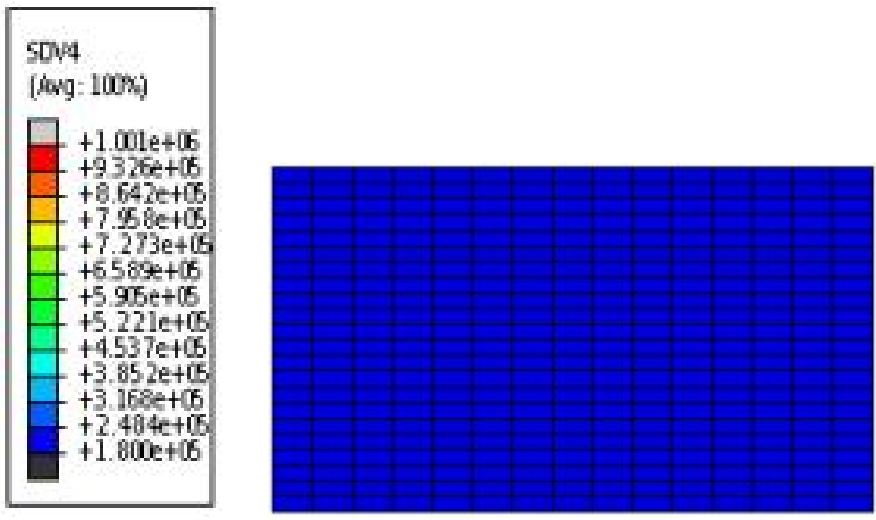

(a) After 1-week stimulation

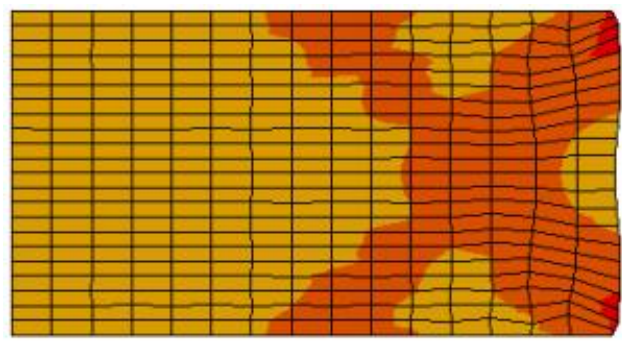

(c) After 3-week stimulation

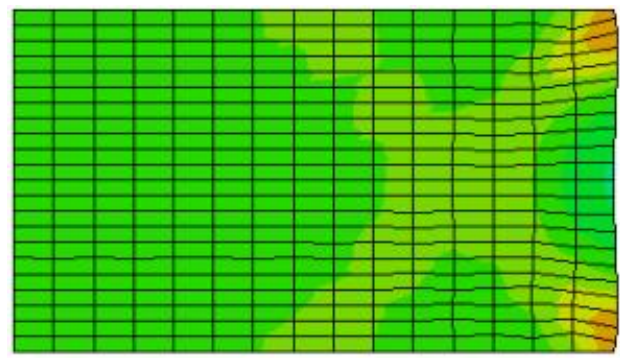

(b) After 2-week stimulation

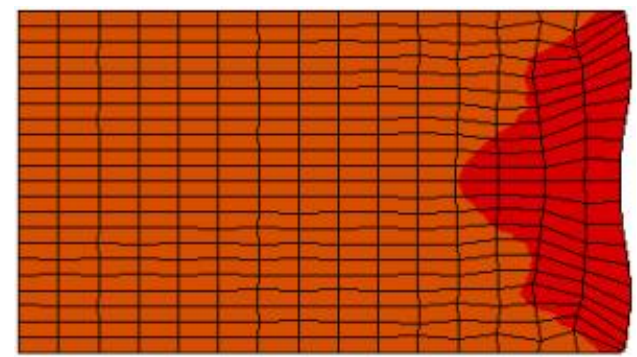

(d) After 4-week stimulation

Figure 6.11: Remodeling of elastic modulus $E_{p}$ in cross section 


\subsubsection{Evaluation of elastic modulus change}

The following tables show the change of the elastic modulus as a result of bioreactor stimulation.

\begin{tabular}{|c|cccc|}
\hline Specimen ID & P580 & P592 & P596 & P602 \\
\hline \hline initial state & 0.500 & 0.800 & 0.896 & 1.047 \\
After 1 week & Not available & Not available & 0.215 & 0.206 \\
After 2 weeks & Not available & Not available & 0.567 & 0.233 \\
After 3 weeks & Not available & Not available & 0.987 & 0.168 \\
After 4 weeks & 0.510 & 1.200 & 0.998 & 0.213 \\
\hline change & increase & increase & increase & decrease \\
\hline
\end{tabular}

Table 6.2: Modification of elastic modulus in 4 week bioreactor stimulation [MPa]

The elastic moduli of three of the four specimens (P580, P592, P596) increase as a result of mechanical stimulation in a bioreactor, respectively by $2 \%, 50 \%$ and $11 \%$.

The amount of elastic modulus increase is different from specimen to specimen. The reason is assumed to be that the states of cells of each specimen differ according to characteristics of the donor such as age, damage state or, gender.

On the contrary, specimen P602 exhibits a decrease of its elastic modulus. The decrease may be caused by enzyme degradation. Thus, the case of P602, enzymes seem to mainly destruct the condensed collagen gel (ground substance) but not to synthesize collagen type II.

\begin{tabular}{|c|ccccc|}
\hline Specimens & P603 & P610 & P611 & P613 & P614 \\
\hline \hline initial state & 0.759 & 0.855 & 0.675 & 0.660 & 0.553 \\
After 2 weeks & 0.458 & 0.426 & 0.374 & 0.250 & 0.560 \\
\hline
\end{tabular}

Table 6.3: Modification of elastic modulus in 2 week bioreactor stimulation [MPa]

Table 6.3 shows the change of elastic modulus in bioreactors that run for two weeks. It shows that the stimulation in the first two weeks generally results in degradation of the material. The phenomenon can be found also in the cases of P596 and P602 in Table 6.2. However, after 
the initial decrease, the Young's modulus of both P596 and P602 start to increase after longer stimulation, respectively two and three weeks. This could indicate a switch point or threshold at which fiber synthesis dominates enzyme degradation. The specimens in Table 6.3 were not stimulated beyond two weeks, and such a switch point was not identified. 


\section{Chapter 7}

\section{Conclusions}

\subsection{Summary}

In the present work, the remodeling of cellular soft tissue due to synthesis of collagen type II fibers was theoretically and experimentally investigated.

The biomechanical phenomenon in a bioreactor is composed of growth and remodeling. If cells seeded in condensed collagen gel are mechanically stimulated, then they synthesize a new material named collagen type II. The synthesis of the new material results in increased mass and volumetric growth of the tissue system. The newly synthesized collagen type II has a fibril structure whose stiffness is about thousand times greater than that of the substrate matrix (condensed collagen gel) and thus reinforces the tissue. Thus, the synthesis brings about a change (remodeling) of material properties.

The collagen type II fibers synthesized by cells make the artificial replacement material both more robust mechanically and medically compatible with the human body.

To describe the general biological phenomenon including change of mass, balance laws for an open system were derived. On the assumption that the solid phase is transversely isotropic and visco-hyperelastic, an appropriate constitutive law was derived. A short time-scale evolution equation depending on the volumetric strain was proposed for the stress decreases as a result of fluid behavior. For the change of material properties resulting from the bioreactor stimulation, a long time-scale evolution equation was proposed. 
For the experimental study, cellular condensed collagen gel specimens were incubated and stimulated mechanically in bioreactors. To follow the change of elastic modulus modification that is regarded as remodeling, a compression test was performed every week. Measurement showed that mass change before and after bioreactor stimulation is not detectable but elastic modulus change is discernible as a long-time-scale evolution. In the first two weeks of bioreactor stimulation, the material is degraded (enzyme degradation), but after the third week, the stiffness begins to increase, since fiber formation dominates.

To validate the proposed theory and to show the difference between short and long time-scale evolution, the constitutive behavior of the tissue was defined in an ABAQUS subroutine used to define a material's mechanical behavior. Corresponding material parameters of both the short time-scale evolution and the remodeling velocity parameter of the long time-scale evolution were determined by curve-fitting.

In summary, the balance laws for an open system were derived and reduced to those of a closed system, which shows the generality of the balance laws for an open system.

It was experimentally verified that mechanical properties of cellular condensed collagen gel change due to cell activity and that such a remodeling effect can be enhanced by means of mechanical stimulation in a bioreactor. With the proposed constitutive equations, (4.3.75) and (4.3.76), the remodeling phenomenon as a consequence of cell activity and the stress relaxation resulting from fluid behavior have been successfully explained. The conversion from an isotropic to a transversely isotropic material due to the modification of Young's modulus was also elucidated.

However, because of the limited number of tests performed, the present study is not sufficient to conclusively explain the observed phenomena in a bioreactor. To state that a bioreactor stimulates cells and the elastic moduli change as a result, more experiments and statistical evaluation are needed. 


\subsection{Future perspectives}

Even though the remodeling phenomenon in a bioreactor and the stress relaxation were experimentally studied and successfully explained with the proposed model, there are still unknown factors that are to be clarified to understand the biomechanical phenomena in a bioreactor, viz:

- Material consolidation: The material of condensed collagen gel becomes compressed due to cyclic loading in a bioreactor and the porosity of the material changes as a function of cyclic loading number. This leads to a change of the diffusion parameters, which is not covered in the present work.

- Enzyme degradation: Due to predamaged cells, the substrate matrix is degraded. How large the enzyme degradation is and how long it occurs are factors to be considered.

- Switch point According to experimental study, it was found that there is a critical moment in time when the enzyme degradation stops and synthesis begins. It should be studied whether degeneration depends on the damaged state of cells or whether it can be avoided if healthy cells are used.

- Optimal stimulation: Material properties, such as Young' modulus, change in bioreactor. If "Autologous Chondrocyte Implantation" is chosen (Subsection 2.5), then the implantation plug should be stimulated/optimized before the implantation operation. According to the experiments, the plug should be stimulated at least three or four weeks. It is still unknown though, whether the plug should be stimulated continuously or several hours a day and with what frequency. To obtain the replacement material parameters similar to the originally undamaged cartilage, optimal mechanical stimulation should be researched.

- Depth of stimulation: Although the replacement material for articular cartilage has the dimension of tissue $\left(10^{-4} \mathrm{~m}-10^{-2} \mathrm{~m}\right)$, we still need to consider cells, as they play a key role for the remodeling. They can be modeled as tiny water balloons whose liquid droplet is enclosed by cortex. Therefore, if they are compressed over their limitation, they burst and die. To keep cells alive and activate their synthesis, the limitation of compression depth and the optimal stress (stimulation) for cells should be studied. 
- Statistics: To explain more exactly the remodeling phenomenon in a bioreactor or in a human body after implantation of cell-seeded tissues, more experiments and statistical data are required.

- Other modifications: In the present study only the change of the elastic modulus $E_{p}$ is assumed. But other elastic material parameters could change due to the synthesized collagen type II fibers. To determine other parameters, further tests are necessary, e.g. a tension test in another direction or a pure shear test (Section 5.3). Additionally, the stress relaxation behavior changes (Table 6.1). The change of diffusion parameters are quite different from that of elastic modulus. It appears that the diffusion parameters are independent of the synthesized collagen type II fiber, as their modification curves are not proportional to those of the elastic modulus (Fig. 6.9). We assume that the diffusion parameters are related to the porosity or the fluid volume fraction.

- Variety of donated material: Material properties of donated material (or competency of donated cells) are strongly dependent on the state of the donor. If donors are classified according to gender, illness, or age and the features of each class are known, the experimental study can be more systematic.

- Proliferation: The number of cells changes due to cell division (reproduction). More detailed descriptions are possible if the change of cell population can be taken into account. 


\section{Appendix A}

\section{Tensors}

This summary on tensor algebra and tensor analysis follows the textbook by Itskov [38], in particular the chapters $1,2,4,5$ and 6 . The notation in this thesis coincides mostly with that in the book. Additionally the textbook by Klingbeil [43] is read as a supplement.

All operations and definitions are described on the three-dimensional Euclidean space denoted by $\mathbb{E}\left(\mathbb{E}^{n}, n=3\right)$.

\section{A.1 Second-order tensors}

\section{Tensor operations}

Let Lin be the set of all linear mappings of one vector into another one in $\mathbb{E}$.

The linear (right) mapping of a vector $\boldsymbol{x}$ into another one $\boldsymbol{y}$ is written as

$$
\boldsymbol{y}=\mathbf{A} \boldsymbol{x}, \quad \mathbf{A} \in \mathbf{L i n}, \boldsymbol{y} \in \mathbb{E}, \forall \boldsymbol{x} \in \mathbb{E} .
$$

The left mapping is defined by

$$
(\boldsymbol{y} \mathbf{A}) \cdot \boldsymbol{x}=\boldsymbol{y} \cdot(\mathbf{A} \boldsymbol{x})=\boldsymbol{y} \mathbf{A} \boldsymbol{x}, \quad \mathbf{A} \in \mathbf{L i n}, \forall \boldsymbol{x}, \boldsymbol{y} \in \mathbb{E} .
$$

A second-order tensor, the tensor product of two vectors $\boldsymbol{a}$ and $\boldsymbol{b}$, is defined by

$$
(\boldsymbol{a} \otimes \boldsymbol{b}) \boldsymbol{x}=\boldsymbol{a}(\boldsymbol{b} \cdot \boldsymbol{x}), \quad \boldsymbol{a}, \boldsymbol{b} \in \mathbb{E}, \forall \boldsymbol{x} \in \mathbb{E} .
$$

The second-order zero tensor $\mathbf{0}$ is defined by

$$
\mathbf{0 x}=\boldsymbol{O}, \quad \forall \boldsymbol{x} \in \mathbb{E},
$$


where $\boldsymbol{O}$ is the zero vector. With the zero tensor $\mathbf{0}$ one can show

$$
\mathbf{A} \mathbf{0}=\mathbf{0 A}=\mathbf{0} \quad \forall \mathbf{A} \in \mathbf{L i n} .
$$

The second-order identity tensor is defined by

$$
\mathbf{I} \boldsymbol{x}=\boldsymbol{x} \mathbf{I}=\boldsymbol{x}, \quad \forall \boldsymbol{x} \in \mathbb{E},
$$

and with it

$$
\mathbf{I A}=\mathbf{A I}=\mathbf{A}, \quad \forall \mathbf{A} \in \mathbf{L i n} .
$$

The transpose tensor $\{\bullet\}^{\mathrm{T}}$ is defined by

$$
\mathbf{A}^{\mathrm{T}} \boldsymbol{x}=\boldsymbol{x} \mathbf{A}, \quad \forall \boldsymbol{x} \in \mathbb{E},
$$

which implies $(\boldsymbol{a} \otimes \boldsymbol{b})^{\mathrm{T}}=\boldsymbol{b} \otimes \boldsymbol{a}$.

A tensor $\mathbf{A}$ is invertible, if for $\boldsymbol{y}=\mathbf{A}^{-1} \boldsymbol{x}$ provided, there exists the inverse tensor $\mathbf{A}^{-1} \in \mathbf{L i n}$ such that

$$
\boldsymbol{x}=\mathbf{A}^{-1} \boldsymbol{y}, \quad \forall \boldsymbol{x} \in \mathbb{E} .
$$

The permutation tensor is defined by

$$
\begin{aligned}
& \mathcal{E}=\mathcal{E}^{i j k} \boldsymbol{g}_{i} \otimes \boldsymbol{g}_{j} \otimes \boldsymbol{g}_{k}=\mathcal{E}_{i j k} \boldsymbol{g}^{i} \otimes \boldsymbol{g}^{j} \otimes \boldsymbol{g}^{k} \\
& \mathcal{E}_{i j k}=\frac{1}{\mathcal{E}^{i j k}}=\left\{\begin{array}{cl}
\sqrt{g} & \text { if } i, j, k=\text { cyclic } 1,2,3 \\
-\sqrt{g} & \text { if } i, j, k=\text { cyclic } 1,3,2 \\
0 & \text { otherwise }
\end{array} \text { where } g^{2}=\operatorname{det}\left(g_{i j}\right) .\right.
\end{aligned}
$$

With transposition and inversion, symmetric, skew-symmetric and orthogonal tensors are defined by:

$$
\begin{aligned}
& \text { Sym }=\left\{\mathbf{M} \in \mathbf{L i n}: \mathbf{M}=\mathbf{M}^{\mathrm{T}}\right\} \\
& \text { Skew }=\left\{\mathbf{W} \in \mathbf{L i n}: \mathbf{W}=-\mathbf{W}^{\mathrm{T}}\right\}, \\
& \text { Orth }=\left\{\mathbf{Q} \in \mathbf{L i n}: \mathbf{Q}=\mathbf{Q}^{-\mathrm{T}}\right\},
\end{aligned}
$$


which are subsets of Lin respectively.

A second-order tensor $\mathbf{A}$ is called positive definite if $\boldsymbol{x} \mathbf{A} \boldsymbol{x}>0$, and positive semi-definite if $\boldsymbol{x} \mathbf{A} \boldsymbol{x} \geq 0, \quad \forall \boldsymbol{x} \neq \boldsymbol{O} \in \mathbb{E}$. Positive definite and positive semi-definite tensors form the sets

$$
\begin{aligned}
& \mathbf{S y m}^{+}=\{\mathbf{M} \in \mathbf{S y m}: \boldsymbol{x} \mathbf{M} \boldsymbol{x}>0, \forall \boldsymbol{x} \neq \boldsymbol{O} \in \mathbb{E}\}, \\
& \mathbf{S y m}_{0}^{+}=\{\mathbf{M} \in \mathbf{S y m}: \boldsymbol{x} \mathbf{M} \boldsymbol{x} \geq 0, \forall \boldsymbol{x} \neq \boldsymbol{O} \in \mathbb{E}\} .
\end{aligned}
$$

The composition $\mathbf{C}=\mathbf{A B}$ of two tensors $\mathbf{A}, \mathbf{B} \in \mathbf{L i n}$ is given by

$$
\mathbf{C} \boldsymbol{x}=\mathbf{A}(\mathbf{B} \boldsymbol{x}), \quad \boldsymbol{y}(\mathbf{A B})=(\boldsymbol{y} \mathbf{A}) \mathbf{B}, \quad \forall \boldsymbol{x}, \boldsymbol{y} \in \mathbb{E} .
$$

If $\mathbf{A}=\boldsymbol{a} \otimes \boldsymbol{b}$ and $\mathbf{B}=\boldsymbol{c} \otimes \boldsymbol{d}$ are given $(\boldsymbol{a}, \boldsymbol{b}, \boldsymbol{c}, \boldsymbol{d} \in \mathbb{E})$

$$
\begin{aligned}
& \mathrm{AB}=(\boldsymbol{a} \otimes \boldsymbol{b})(\boldsymbol{c} \otimes \boldsymbol{d})=\boldsymbol{b} \cdot \boldsymbol{c}(\boldsymbol{a} \otimes \boldsymbol{d}), \\
& \mathbf{A}: \mathbf{B}=(\boldsymbol{a} \otimes \boldsymbol{b}):(\boldsymbol{c} \otimes \boldsymbol{d})=(\boldsymbol{a} \cdot \boldsymbol{c})(\boldsymbol{b} \cdot \boldsymbol{d}) \\
& =(\boldsymbol{a} \otimes \boldsymbol{b}): \mathrm{B}=\boldsymbol{a B} \boldsymbol{b}=\boldsymbol{b} \mathrm{B}^{\mathrm{T}} \boldsymbol{a}=(\boldsymbol{b} \otimes \boldsymbol{a}):(\boldsymbol{d} \otimes \boldsymbol{c}) .
\end{aligned}
$$

The norm and the trace of a second-order $\mathbf{A}$ tensor are defined by

$$
\|\mathbf{A}\|=(\mathbf{A}: \mathbf{A})^{1 / 2}, \quad \operatorname{tr} \mathbf{A}=\mathbf{A}: \mathbf{I} .
$$

The principal invariants and the determinant of a second-order tensor $\mathbf{A}$ are

$$
\begin{aligned}
& \mathrm{I}_{\mathbf{A}}=\operatorname{tr} \mathbf{A} \\
& \mathrm{II}_{\mathbf{A}}=\frac{1}{2}\left[(\operatorname{tr} \mathbf{A})^{2}-\operatorname{tr} \mathbf{A}^{2}\right] \\
& \mathrm{III}_{\mathbf{A}}=\operatorname{det} \mathbf{A}=\frac{1}{3}\left[\operatorname{tr} \mathbf{A}^{3}-\frac{3}{2} \operatorname{tr} \mathbf{A}^{2} \operatorname{tr} \mathbf{A}+\frac{1}{2}(\operatorname{tr} \mathbf{A})^{3}\right] .
\end{aligned}
$$

With the help of the determinant the invertible, the proper orthogonal and the unimodular tensors are defined

$$
\begin{aligned}
& \text { Inv }=\{\mathbf{A} \in \mathbf{L i n}: \operatorname{det} \mathbf{A} \neq 0\} \\
& \text { Sorth }=\{\mathbf{Q} \in \text { Orth }: \operatorname{det} \mathbf{Q}=1\} \\
& \text { Uinm }=\{\mathbf{H} \in \mathbf{L i n}: \operatorname{det} \mathbf{H}= \pm 1\}
\end{aligned}
$$

The adjugate and the cofactor of an invertible tensor $\mathbf{A} \in \mathbf{I n v}$

$$
\operatorname{adj} \mathbf{A}=\mathbf{A}^{-1} \operatorname{det} \mathbf{A}, \quad \operatorname{cof} \mathbf{A}=\mathbf{A}^{-\mathrm{T}} \operatorname{det} \mathbf{A}=(\operatorname{adj} \mathbf{A})^{\mathrm{T}} .
$$




\section{Component representation}

Let $\mathcal{G}=\left\{\boldsymbol{g}_{1}, \boldsymbol{g}_{2}, \boldsymbol{g}_{3}\right\}$ and $\mathcal{G}^{\prime}=\left\{\boldsymbol{g}^{1}, \boldsymbol{g}^{2}, \boldsymbol{g}^{3}\right\}$ be dual bases in $\mathbb{E}$. A second-order tensor $\mathbf{A}$ can be respresented by

$$
\begin{aligned}
& \mathbf{A}=A^{i j} \boldsymbol{g}_{i} \otimes \boldsymbol{g}_{j}=A_{i j} \boldsymbol{g}^{i} \otimes \boldsymbol{g}^{j}=A_{\cdot j}^{i} \boldsymbol{g}_{i} \otimes \boldsymbol{g}^{j}=A_{i}{ }^{j} \boldsymbol{g}^{i} \otimes \boldsymbol{g}_{j} \\
& A^{i j}=\boldsymbol{g}^{i} \mathbf{A} \boldsymbol{g}^{j}, \quad A_{i j}=\boldsymbol{g}_{i} \mathbf{A} \boldsymbol{g}_{j}, \quad A_{\cdot j}^{i}=\boldsymbol{g}^{i} \mathbf{A} \boldsymbol{g}_{j}, \quad A_{i}{ }^{j}=\boldsymbol{g}_{i} \mathbf{A} \boldsymbol{g}^{j} .
\end{aligned}
$$

The identity tensor $\mathbf{I}$ can be expressed

$$
\begin{aligned}
& \mathbf{I}=g_{i j} \boldsymbol{g}^{i} \otimes \boldsymbol{g}^{j}=g^{i j} \boldsymbol{g}_{i} \otimes \boldsymbol{g}_{j}=g_{i .}^{j} \boldsymbol{g}^{i} \otimes \boldsymbol{g}_{j}=g_{\cdot j}^{i} \boldsymbol{g}_{i} \otimes \boldsymbol{g}^{j}=\boldsymbol{g}_{i} \otimes \boldsymbol{g}^{i}=\boldsymbol{g}^{i} \otimes \boldsymbol{g}_{i}, \\
& I^{i j}=\boldsymbol{g}^{i} \mathbf{I} \boldsymbol{g}^{j}=\boldsymbol{g}^{i} \cdot \boldsymbol{g}^{j}=g^{i j}, \quad I_{i j}=\boldsymbol{g}_{i} \mathbf{I} \boldsymbol{g}_{j}=\boldsymbol{g}_{i} \cdot \boldsymbol{g}_{j}=g_{i j}, \\
& I_{\cdot j}^{i}=\boldsymbol{g}^{i} \mathbf{I} \boldsymbol{g}_{j}=\boldsymbol{g}^{i} \cdot \boldsymbol{g}_{j}=g_{\cdot j}^{i}=\delta_{\cdot j}^{i}, \quad I_{j \cdot}^{i}=\boldsymbol{g}_{j} \mathbf{I} \boldsymbol{g}^{i}=\boldsymbol{g}_{j} \cdot \boldsymbol{g}^{i}=g_{j}{ }^{i}=\delta_{j .}^{i}, \\
& \delta_{\cdot j}^{i}=\delta_{j}^{i}=\delta_{j}^{i}=\delta_{i}^{j}=g_{j}^{i}=g_{i}^{j} .
\end{aligned}
$$

The composition and the scalar product of any two second-order tensors are

$$
\begin{aligned}
\mathbf{A B} & =A^{i k} B_{k \cdot}^{j} \boldsymbol{g}_{i} \otimes \boldsymbol{g}_{j}=A_{\cdot k}^{i} B^{k j} \boldsymbol{g}_{i} \otimes \boldsymbol{g}_{j}=A_{i \cdot}{ }^{k} B_{k}{ }^{j} \boldsymbol{g}^{i} \otimes \boldsymbol{g}_{j}=A_{i k} B^{k j} \boldsymbol{g}^{i} \otimes \boldsymbol{g}_{j} \\
& =A_{i \cdot}{ }^{k} B_{k j} \boldsymbol{g}^{i} \otimes \boldsymbol{g}^{j}=A_{i k} B_{\cdot j}^{k} \boldsymbol{g}^{i} \otimes \boldsymbol{g}^{j}=A^{i k} B_{k j} \boldsymbol{g}_{i} \otimes \boldsymbol{g}^{j}=A_{\cdot k}^{i} B_{\cdot j}^{k} \boldsymbol{g}_{i} \otimes \boldsymbol{g}^{j}, \\
\mathbf{A}: \mathbf{B} & =A_{i j} B^{i j}=A^{i j} B_{i j}=A_{\cdot j}^{i} B_{i \cdot}{ }^{j}=A_{i \cdot}{ }^{j} B_{\cdot j}^{i}
\end{aligned}
$$

and the determinant of a second-order tensor $\mathbf{A}$ is

$$
\begin{aligned}
\operatorname{det} \mathbf{A} & =\left|A_{\cdot j}^{i}\right|=\left|A^{i k} g_{k j}\right|=\left|A^{i k}\right|\left|g_{k j}\right|=\left|A^{i k}\right| g^{2} \\
& =\left|A_{i \cdot}^{j}\right|=\left|A_{i k} g^{k j}\right|=\left|A_{i k}\right|\left|g^{k j}\right|=\left|A_{i k}\right| g^{-2}, \quad g^{2}=\left|g_{k j}\right| .
\end{aligned}
$$

The scalar product between the permutation tensor $\mathcal{E}$ and a any second-order tensor $\mathbf{A}$ is

$$
\mathcal{E}: \mathbf{A}=\mathcal{E}_{i j k} \boldsymbol{g}^{i} \otimes \boldsymbol{g}^{j} \otimes \boldsymbol{g}^{k}: A_{l m} \boldsymbol{g}^{l} \otimes \boldsymbol{g}^{m}=\mathcal{E}_{i j k} A_{l m} \boldsymbol{g}^{i} g^{j l} g^{k m}=\mathcal{E}_{i j k} A^{j k} \boldsymbol{g}^{i}
$$

and if $\mathbf{T}$ is a symmetric tensor $(\mathbf{T} \in \mathbf{S y m})$,

$$
\begin{aligned}
& \mathcal{E}: \mathbf{T}=\mathcal{E}: \mathbf{T}^{\mathrm{T}}=-\mathcal{E}: \mathbf{T} \rightarrow \mathcal{E}: 2 \mathbf{T}=\boldsymbol{O} \rightarrow \mathcal{E}: \mathbf{T}=\boldsymbol{O}, \\
& \text { for } i=1, \quad \mathcal{E}_{1 j k} T^{j k}=\mathcal{E}_{123} T^{23}+\mathcal{E}_{132} T^{32}=\mathcal{E}_{123}\left(T^{23}-T^{32}\right)=0 .
\end{aligned}
$$


The vector product of two vectors and of two tensors are

$$
\begin{aligned}
& \boldsymbol{a} \times \boldsymbol{b}=\mathcal{E}:(\boldsymbol{a} \otimes \boldsymbol{b})=\mathcal{E}_{i j k} a^{j} b^{k} \boldsymbol{g}^{i} \\
& \mathbf{A} \times \mathbf{B} \stackrel{\text { def }}{=} \mathcal{E}:\left(\mathbf{A B}^{\mathrm{T}}\right)=\mathcal{E}_{i j k}\left(\mathbf{A B}^{\mathrm{T}}\right)^{j k} \boldsymbol{g}^{i}=\mathcal{E}_{i j k} A^{j l} B^{k l} \boldsymbol{g}^{i} .
\end{aligned}
$$

\section{Decomposition of second-order tensors}

Every second-order tensor can be decomposed into a symmetric and a skew-symmetric part by

$$
\mathbf{A}=\operatorname{sym} \mathbf{A}+\text { skew } \mathbf{A}, \quad \operatorname{sym} \mathbf{A}=\frac{1}{2}\left(\mathbf{A}+\mathbf{A}^{\mathrm{T}}\right), \quad \text { skew } \mathbf{A}=\frac{1}{2}\left(\mathbf{A}-\mathbf{A}^{\mathrm{T}}\right),
$$

as well as a spherical and a deviatoric part by

$$
\mathbf{A}=\operatorname{sph} \mathbf{A}+\operatorname{dev} \mathbf{A}, \quad \operatorname{sph} \mathbf{A}=\mathbf{I} \frac{1}{3} \operatorname{tr} \mathbf{A}, \quad \operatorname{dev} \mathbf{A}=\mathbf{A}-\mathbf{I} \frac{1}{3} \operatorname{tr} \mathbf{A} .
$$

We consider the case of $n$ simple eigenvalues of a second-order A. Solving the equation systems $\mathbf{A} \boldsymbol{a}=\lambda \boldsymbol{a}$ (eigenvalue problem) one obtains for every simple eigenvalues $\lambda_{i}$ the components of right eigenvector $\boldsymbol{a}_{i}$ and the components of the left eigenvector $\boldsymbol{b}_{i}(i=1,2,3)$

$$
\mathbf{A}=\sum_{i}^{3} \lambda_{i} \boldsymbol{a}_{i} \otimes \boldsymbol{b}_{i} \quad \text { (spectral decomposition for diagonalizable tensors). }
$$

Symmetric second-order tensors possess throughout real eigenvalues and allow

$$
\mathbf{M}=\sum_{i=1}^{n_{e}} \lambda_{i} \sum_{j=1}^{m_{a}} \boldsymbol{p}_{i}^{(j)} \otimes \boldsymbol{p}_{i}^{(j)}=\sum_{i=1}^{n_{e}} \lambda_{i} \mathbf{p}_{i}, \quad \mathbf{M} \in \mathbf{S y m}
$$

where $n_{e}$ is the number of distinct eigenvalues of $\lambda_{i}, m_{a}$ denotes the algebraic multiplicity, $\boldsymbol{p}_{i}^{(k)}$ are the eigenvectors characterized by $\boldsymbol{p}_{i}^{(k)} \cdot \boldsymbol{p}_{j}^{(l)}=\delta_{i j} \delta^{k l}$ and the second-order tensors $\mathbf{p}_{i}=$ $\sum_{j=1}^{m_{a}} \boldsymbol{p}_{i}^{(j)} \otimes \boldsymbol{p}_{i}^{(j)}$ are referred to as eigenprojections.

Positive definite tensors have throughout positive eigenvalues and for positive semi-definite tensors zero may be one or some eigenvalues. Positive semi-definite symmetric tensors can be represented by the composition

$$
\mathbf{M}=\mathbf{A} \mathbf{A}^{\mathrm{T}}, \quad \mathbf{M} \in \mathbf{S y m}_{0}^{+}, \mathbf{A} \in \mathbf{L i n} .
$$




\section{A.2 Fourth-order tensors}

\section{Tensor operations}

Fourth-order tensors are the elements of the set Lin of all linear mappings which maps one second-order tensor into another one. The right and the left mapping are

$$
\mathbf{Y}=\mathbb{A}: \mathbf{X}, \quad(\mathbf{Y}: \mathbb{A}): \mathbf{X}=\mathbf{Y}:(\mathbb{A}: \mathbf{X}), \quad \mathbb{A} \in \operatorname{Lin}, \quad \forall \mathbf{X}, \mathbf{Y} \in \mathbf{L i n},
$$

respectively.

Fourth-order tensors are defined by tensor products of two second-order tensors

$\mathbf{A} \otimes \mathbf{B}: \mathbf{X} \stackrel{\text { def }}{=} \mathbf{A X B}$

$\mathbf{A} \odot \mathbf{B}: \mathbf{X} \stackrel{\text { def }}{=} \mathbf{A}(\mathbf{B}: \mathbf{X}), \quad \mathbf{A}, \mathbf{B} \in \mathbf{L i n}, \forall \mathbf{X} \in \mathbf{L i n}$.

For the left mapping we have

$$
\begin{aligned}
& \mathbf{Y}: \mathbf{A} \otimes \mathbf{B}=\mathbf{A}^{\mathrm{T}} \mathbf{Y} \mathbf{B}^{\mathrm{T}}, \\
& \mathbf{Y}: \mathbf{A} \odot \mathbf{B}=(\mathbf{Y}: \mathbf{A}) \mathbf{B}, \quad \mathbf{A}, \mathbf{B} \in \mathbf{L i n}, \forall \mathbf{Y} \in \mathbf{L i n} .
\end{aligned}
$$

Two contraction operations of fourth-order tensors are defined by

$$
\begin{aligned}
& \mathbf{A} \odot \mathbf{B} \bullet \circ \mathbf{X} \stackrel{\text { def }}{=} \mathbf{A}^{\mathrm{T}} \otimes \mathbf{B}: \mathbf{X}=\mathbf{A}^{\mathrm{T}} \mathbf{X} \mathbf{B}, \\
& \mathbf{A} \odot \mathbf{B} \circ \bullet \mathbf{X} \stackrel{\text { def }}{=} \mathbf{A} \otimes \mathbf{B}^{\mathrm{T}}: \mathbf{X}=\mathbf{A} \mathbf{X} \mathbf{B}^{\mathrm{T}}, \quad \mathbf{A}, \mathbf{B} \in \mathbf{L i n}, \forall \mathbf{X} \in \mathbf{L i n} .
\end{aligned}
$$

The compositions of two fourth-order tensor are defined by

$$
\begin{aligned}
& (\mathbb{A}: \mathbb{B}): \mathbf{X} \stackrel{\text { def }}{=} \mathbb{A}:(\mathbb{B}: \mathbf{X}), \quad \mathbb{A}, \mathbb{B} \in \mathbb{L i n}, \quad \forall \mathbf{X} \in \text { Lin, } \\
& \mathbf{Y}:(\mathbb{A}: \mathbb{B}) \stackrel{\text { def }}{=}(\mathbf{Y}: \mathbb{A}): \mathbb{B}, \quad \mathbb{A}, \mathbb{B} \in \operatorname{Lin}, \quad \forall \mathbf{Y} \in \mathbf{L i n} .
\end{aligned}
$$

The composition of a fourth-order tensor with two second-order tensor is defined by

$$
(\mathbf{A D B}): \mathbf{X}=\mathbf{A}(\mathbb{D}: \mathbf{X}) \mathbf{B}, \quad \mathbf{A}, \mathbf{B} \in \mathbf{L i n}, \mathbb{D} \in \operatorname{Lin}, \forall \mathbf{X} \in \mathbf{L i n}
$$

The two transposition operations of fourth-order tensors $(\bullet)^{\mathrm{T}}$ and $(\bullet)^{\mathrm{t}}$ are defined by

$$
\mathbb{A}^{\mathrm{T}}: \mathbf{X} \stackrel{\text { def }}{=} \mathbf{X}: \mathbb{A}, \mathbb{A}^{\mathrm{t}}: \mathbf{X} \stackrel{\text { def }}{=} \mathbb{A}: \mathbf{X}^{\mathrm{T}}, \mathbf{Y}: \mathbb{A}^{\mathrm{t}}=(\mathbf{Y}: \mathbb{A})^{\mathrm{T}}, \mathbb{A} \in \operatorname{Lin}, \forall \mathbf{X}, \mathbf{Y} \in \mathbf{L i n}
$$


and based on these, a symmetrization operation $(\bullet)^{\mathrm{S}}$ is introduced

$$
\mathbb{A}^{\mathrm{S}}=\frac{1}{2}\left(\mathbb{A}+\mathbb{A}^{\mathrm{t}}\right)
$$

This yields for right and the left mapping

$$
\begin{aligned}
\mathbb{A}^{\mathrm{S}}: \mathbf{X} & =\frac{1}{2}\left(\mathbb{A}+\mathbb{A}^{\mathrm{t}}\right): \mathbf{X}=\frac{1}{2} \mathbb{A}: \mathbf{X}+\frac{1}{2} \mathbb{A}^{\mathrm{t}}: \mathbf{X}=\frac{1}{2} \mathbb{A}: \mathbf{X}+\frac{1}{2} \mathbb{A}: \mathbf{X}^{\mathrm{T}} \\
& =\mathbb{A}: \frac{1}{2}\left(\mathbf{X}+\mathbf{X}^{\mathrm{T}}\right)=\mathbb{A}: \operatorname{sym} \mathbf{X} \\
\mathbf{Y}: \mathbb{A}^{\mathrm{S}} & =\mathbf{Y}: \frac{1}{2}\left(\mathbb{A}+\mathbb{A}^{\mathrm{t}}\right)=\frac{1}{2} \mathbf{Y}: \mathbb{A}+\frac{1}{2} \mathbf{Y}: \mathbb{A}^{\mathrm{t}}=\frac{1}{2} \mathbf{Y}: \mathbb{A}+\frac{1}{2}(\mathbf{Y}: \mathbb{A})^{\mathrm{T}} \\
& =\operatorname{sym}(\mathbf{Y}: \mathbb{A}), \quad \forall \mathbf{X}, \mathbf{Y} \in \mathbf{L i n} .
\end{aligned}
$$

Fourth-order zero $\mathbb{O}$ and identity tensor II

$\mathbb{0}: \mathbf{X}=\mathbf{0}, \quad \mathbb{I}: \mathbf{X}=(\mathbf{I} \otimes \mathbf{I}): \mathbf{X}=\mathbf{I X I}=\mathbf{X}, \quad \forall \mathbf{X} \in \mathbf{L i n}$.

$\mathbb{I}^{\mathrm{T}}=(\mathbf{I} \otimes \mathbf{I})^{\mathrm{T}}=\mathbf{I}^{\mathrm{T}} \otimes \mathbf{I}^{\mathrm{T}}=\mathbf{I} \otimes \mathbf{I}=\mathbb{I}$.

$\mathbb{I}: \mathbf{X}=\mathbf{X}, \quad \mathbf{X}: \mathbb{I}=\mathbb{I}^{\mathrm{T}}: \mathbf{X}=\mathbf{X}, \quad \forall \mathbf{X} \in \mathbf{L i n}$.

$\mathbf{A I}=\mathbf{A}(\mathbf{I} \otimes \mathbf{I})=(\mathbf{A I}) \otimes \mathbf{I}=\mathbf{A} \otimes \mathbf{I}$.

Transpositions of a fourth-order tensor $\{\bullet\}^{\mathrm{T}}$ and $\{\bullet\}^{\mathrm{t}}$

$(\mathbf{A} \otimes \mathbf{B})^{\mathrm{T}}=\mathbf{A}^{\mathrm{T}} \otimes \mathbf{B}^{\mathrm{T}}, \quad(\mathbf{A} \odot \mathbf{B})^{\mathrm{T}}=\mathbf{B} \odot \mathbf{A}, \quad(\mathbf{A} \odot \mathbf{B})^{\mathrm{t}}=\mathbf{A} \odot \mathbf{B}^{\mathrm{T}}, \quad \forall \mathbf{A}, \mathbf{B} \in \mathbf{L i n}$.

$(\boldsymbol{a} \otimes \boldsymbol{b} \otimes \boldsymbol{c} \otimes \boldsymbol{d})^{\mathrm{T}}=\boldsymbol{b} \otimes \boldsymbol{a} \otimes \boldsymbol{d} \otimes \boldsymbol{c}, \quad \forall \boldsymbol{a}, \boldsymbol{b}, \boldsymbol{c}, \boldsymbol{d} \in \mathbb{E}$.

$(\boldsymbol{a} \otimes \boldsymbol{b} \otimes \boldsymbol{c} \otimes \boldsymbol{d})^{\mathrm{t}}=\boldsymbol{a} \otimes \boldsymbol{c} \otimes \boldsymbol{b} \otimes \boldsymbol{d}, \quad \forall \boldsymbol{a}, \boldsymbol{b}, \boldsymbol{c}, \boldsymbol{d} \in \mathbb{E}$.

$\mathbb{A}^{\mathrm{TT}}=\mathbb{A}, \quad \mathbb{A}^{\mathrm{tt}}=\mathbb{A}, \quad \forall \mathbb{A} \in$ Lin.

The transposition tensor $\mathbb{T}=\mathbb{I}^{\mathrm{t}}$ maps a second-order tensor to its transpose

$$
\mathrm{T}: \mathbf{X}=\mathbf{X}^{\mathrm{T}}, \quad \mathbf{Y}: \mathbb{T}=\mathbf{Y}^{\mathrm{T}}, \quad \forall \mathbf{X}, \mathbf{Y} \in \mathbf{L i n} .
$$


The decomposition of second-order tensor can be represented by fourth-order projection tensors

$$
\operatorname{sph} \mathbf{A}=\mathbf{I} \frac{1}{3} \operatorname{tr} \mathbf{A}=\mathbb{P}_{\mathrm{s}}: \mathbf{A}, \quad \operatorname{dev} \mathbf{A}=\mathbf{A}-\mathbf{I} \frac{1}{3} \operatorname{tr} \mathbf{A}=\mathbb{P}_{\mathrm{d}}: \mathbf{A}
$$

where $\mathbb{P}_{\mathrm{s}}$ and $\mathbb{P}_{\mathrm{d}}$ are spherical and deviatoric projection tensor, respectively

$$
\mathbb{P}_{\mathrm{s}}=\frac{1}{3} \mathbf{I} \odot \mathbf{I}, \quad \mathbb{P}_{\mathrm{d}}=\mathbf{I}-\frac{1}{3} \mathbf{I} \odot \mathbf{I} .
$$

Super-symmetric tensors $\mathbb{S}$ form a subset Ssym of Lin as

$$
\operatorname{Ssym}=\left\{\mathbb{S} \in \operatorname{Lin}: \mathbb{S}=\mathbb{S}^{\mathrm{T}} \wedge \mathbb{S}=\mathbb{S}^{t}\right\}
$$

The super-symmetric identity tensor $\mathbb{I}^{\mathrm{S}}$ maps any second-order tensor into its symmetric part:

$$
\mathbb{I}^{\mathrm{S}}: \mathbf{X}=\mathbf{X}: \mathbb{I}^{\mathrm{S}}=\operatorname{sym} \mathbf{X}, \quad \forall \mathbf{X} \in \mathbf{L i n} .
$$

\section{Component representation}

$$
\begin{aligned}
& (\boldsymbol{a} \otimes \boldsymbol{b}) \otimes(\boldsymbol{c} \otimes \boldsymbol{d})=\boldsymbol{a} \otimes \boldsymbol{b} \otimes \boldsymbol{c} \otimes \boldsymbol{d} . \\
& (\boldsymbol{a} \otimes \boldsymbol{b}) \odot(\boldsymbol{c} \otimes \boldsymbol{d})=\boldsymbol{a} \otimes \boldsymbol{c} \otimes \boldsymbol{d} \otimes \boldsymbol{b} .
\end{aligned}
$$

For an implementation of constitutive equations into finite element codes, the components of a fourth-order tensor needs to be expressed with the basis $\left(\boldsymbol{e}_{i} \otimes \boldsymbol{e}_{j}\right) \odot\left(\boldsymbol{e}_{k} \otimes \boldsymbol{e}_{l}\right)$, where 
$\mathcal{E}=\left\{\boldsymbol{e}_{1}, \boldsymbol{e}_{2}, \boldsymbol{e}_{3}\right\}$ denotes an orthonormal basis of $\mathbb{E}$.

$\mathbf{A} \otimes \mathbf{B}=\boldsymbol{a} \otimes \boldsymbol{b} \otimes \boldsymbol{c} \otimes \boldsymbol{d} \rightarrow(\mathbf{A} \otimes \mathbf{B})^{i j k l}=A^{i j} B^{k l}$,

$\mathbf{A} \odot \mathbf{B}=\boldsymbol{a} \otimes \boldsymbol{c} \otimes \boldsymbol{d} \otimes \boldsymbol{b} \rightarrow(\mathbf{A} \odot \mathbf{B})^{i j k l}=A^{i l} B^{j k}$,

$(\mathbf{A} \otimes \mathbf{B})^{\mathrm{T}}=\boldsymbol{b} \otimes \boldsymbol{a} \otimes \boldsymbol{d} \otimes \boldsymbol{c} \rightarrow\left[(\mathbf{A} \otimes \mathbf{B})^{\mathrm{T}}\right]^{i j k l}=A^{j i} B^{l k}$,

$(\mathbf{A} \otimes \mathbf{B})^{\mathrm{t}}=\boldsymbol{a} \otimes \boldsymbol{c} \otimes \boldsymbol{b} \otimes \boldsymbol{d} \rightarrow\left[(\mathbf{A} \otimes \mathbf{B})^{\mathrm{t}}\right]^{i j k l}=A^{i k} B^{j l}$,

$(\mathbf{A} \otimes \mathbf{B})^{\mathrm{S}}=\frac{1}{2}(\boldsymbol{a} \otimes \boldsymbol{b} \otimes \boldsymbol{c} \otimes \boldsymbol{d}+\boldsymbol{a} \otimes \boldsymbol{c} \otimes \boldsymbol{b} \otimes \boldsymbol{d})$

$$
\rightarrow\left[(\mathbf{A} \otimes \mathbf{B})^{\mathrm{S}}\right]^{i j k l}=\frac{1}{2}\left(A^{i j} B^{k l}+A^{i k} B^{j l}\right),
$$

$(\mathbf{A} \odot \mathbf{B})^{\mathrm{T}}=\boldsymbol{c} \otimes \boldsymbol{a} \otimes \boldsymbol{b} \otimes \boldsymbol{d} \rightarrow\left[(\mathbf{A} \odot \mathbf{B})^{\mathrm{T}}\right]^{i j k l}=A^{j k} B^{i l}$,

$(\mathbf{A} \odot \mathbf{B})^{\mathrm{t}}=\boldsymbol{a} \otimes \boldsymbol{d} \otimes \boldsymbol{c} \otimes \boldsymbol{b} \rightarrow\left[(\mathbf{A} \odot \mathbf{B})^{\mathrm{t}}\right]^{i j k l}=A^{i l} B^{k j}$,

$(\mathbf{A} \odot \mathbf{B})^{\mathrm{S}}=\frac{1}{2}(\boldsymbol{a} \otimes \boldsymbol{c} \otimes \boldsymbol{d} \otimes \boldsymbol{b}+\boldsymbol{a} \otimes \boldsymbol{d} \otimes \boldsymbol{c} \otimes \boldsymbol{b})$

$$
\rightarrow\left[(\mathbf{A} \odot \mathbf{B})^{\mathrm{S}}\right]^{i j k l}=\frac{1}{2}\left(A^{i l} B^{j k}+A^{i l} B^{k j}\right)
$$

where $\mathbf{A}=\boldsymbol{a} \otimes \boldsymbol{b}=A^{i j} \boldsymbol{e}_{i} \otimes \boldsymbol{e}_{j}$ and $\mathbf{B}=\boldsymbol{c} \otimes \boldsymbol{d}=B^{i j} \boldsymbol{e}_{i} \otimes \boldsymbol{e}_{j}$

\section{A.3 Differentiation with respect to a tensor}

\section{Scalar-valued tensor function}

The derivative of a scalar-valued tensor function $f(\mathbf{A}):$ Lin $\rightarrow \mathbb{R}$ ( $\mathbb{R}$ is the set of all real numbers) with respect to the tensor $\mathbf{A}$ is defined by

$$
\begin{aligned}
& \left.\frac{d}{d t} f(\mathbf{A}+t \mathbf{X})\right|_{t=0}=f(\mathbf{A})_{, \mathbf{A}}: \mathbf{X}, \quad \forall \mathbf{X} \in \mathbf{L i n}, \\
& f(\mathbf{A})_{, \mathbf{A}}=\frac{\partial f}{\partial A^{i j}} \boldsymbol{g}^{i} \otimes \boldsymbol{g}^{j}=\frac{\partial f}{\partial A_{i j}} \boldsymbol{g}_{i} \otimes \boldsymbol{g}_{j}=\frac{\partial f}{\partial A_{i}{ }^{j}} \boldsymbol{g}_{i} \otimes \boldsymbol{g}^{j}=\frac{\partial f}{\partial A_{\cdot j}^{i}} \boldsymbol{g}^{i} \otimes \boldsymbol{g}_{j}
\end{aligned}
$$


where $f(\mathbf{A})_{, \mathbf{A}} \in \mathbf{L i n}$.

$$
\begin{aligned}
& f(\mathbf{M})_{, \mathbf{M}}=\operatorname{sym}\left[f(\mathbf{M})_{, \mathbf{M}}\right], \quad \mathbf{M}=\operatorname{sym} \mathbf{A} \in \mathbf{S y m}, \quad \mathbf{A} \in \mathbf{L i n}, \\
& \left(\operatorname{tr} \mathbf{A}^{k}\right)_{, \mathbf{A}}=k\left(\mathbf{A}^{k-1}\right)^{\mathrm{T}} \\
& I_{\mathbf{A}, \mathbf{A}}^{(n)}=I_{\mathbf{A}}^{(n)} \mathbf{A}^{-\mathrm{T}}, \quad \mathbf{A} \in \mathbf{I n v}^{n} .
\end{aligned}
$$

\section{Tensor-valued tensor function}

The derivative of a tensor-valued tensor function $\mathbf{f}(\mathbf{A}):$ Lin $\rightarrow$ Lin with respect to the tensor A is defined by

$$
\begin{aligned}
& \left.\frac{d}{d t} \mathbf{f}(\mathbf{A}+t \mathbf{X})\right|_{t=0}=\mathbf{f}(\mathbf{A})_{, \mathbf{A}}: \mathbf{X}, \quad \forall \mathbf{X} \in \mathbf{L i n}, \\
& \begin{aligned}
\mathbf{f}(\mathbf{A})_{, \mathbf{A}} & =\frac{\partial f_{\cdot j}^{i}}{\partial A^{k l}} \boldsymbol{g}_{i} \otimes \boldsymbol{g}^{k} \otimes \boldsymbol{g}^{l} \otimes \boldsymbol{g}^{j}=\frac{\partial f_{\cdot j}^{i}}{\partial A_{k l}} \boldsymbol{g}_{i} \otimes \boldsymbol{g}_{k} \otimes \boldsymbol{g}_{l} \otimes \boldsymbol{g}^{j} \\
& =\frac{\partial f_{\cdot j}^{i}}{\partial A_{k}^{l}} \boldsymbol{g}_{i} \otimes \boldsymbol{g}_{k} \otimes \boldsymbol{g}^{l} \otimes \boldsymbol{g}^{j}=\frac{\partial f_{\cdot j}^{i}}{\partial A_{\cdot l}^{k}} \boldsymbol{g}_{i} \otimes \boldsymbol{g}^{k} \otimes \boldsymbol{g}_{l} \otimes \boldsymbol{g}^{j}
\end{aligned}
\end{aligned}
$$

where $\mathbf{f}(\mathbf{A})_{, \mathbf{A}} \in$ Lin. For symmetric second-order tensors

$$
\mathbf{f}(\mathbf{M})_{, \mathbf{M}}=\left[\mathbf{f}(\mathbf{A})_{\mathbf{A}}\right]^{\mathrm{S}}, \quad \mathbf{M}=\operatorname{sym} \mathbf{A} \in \mathbf{S y m}, \mathbf{A} \in \mathbf{L i n}
$$

The derivatives with respect to a tensor obey the following rules of differentiation

$$
\begin{aligned}
& \mathbf{A}_{, \mathbf{A}}^{k}=\sum_{i=0}^{k-1} \mathbf{A}^{i} \otimes \mathbf{A}^{k-1-i} \quad(k \geq 1), \quad \mathbf{A}_{, \mathbf{A}}=\mathbb{I}, \\
& \mathbf{A}_{, \mathbf{A}}^{\mathrm{T}}=\mathbb{T}, \quad \mathbf{A}_{, \mathbf{A}}^{-1}=-\mathbf{A}^{-1} \otimes \mathbf{A}^{-1}, \\
& \quad=(\mathbf{I} \otimes \mathbf{I}) \mathbf{A}^{-1}-\left(\mathbf{A A}^{-1}\right) \otimes \mathbf{A}^{-1}=\mathbf{I} \otimes\left(\mathbf{I} \mathbf{A}^{-1}\right)-\mathbf{I} \otimes \mathbf{A}^{-1}=\mathbb{0} \\
& g_{, \mathbf{B}}=g_{, \mathbf{A}}: \mathbf{A}_{, \mathbf{B}}, \quad \mathbf{G}_{, \mathbf{B}}=\mathbf{G}_{, \mathbf{A}}: \mathbf{A}_{, \mathbf{B}}, \\
& (g \mathbf{G})_{, \mathbf{A}}=\mathbf{G} \odot \mathbf{A}_{, \mathbf{A}}+g \mathbf{G}_{, \mathbf{A}}, \\
& (\mathbf{G} \mathbf{H})_{, \mathbf{A}}=\mathbf{G} \mathbf{G}_{, \mathbf{A}} \mathbf{H}+\mathbf{G H}_{, \mathbf{A}}, \\
& (\mathbf{G}: \mathbf{H})_{, \mathbf{A}}=\mathbf{H}: \mathbf{G}_{, \mathbf{A}}+\mathbf{G}: \mathbf{H}_{, \mathbf{A}}
\end{aligned}
$$


where $g=\hat{g}(\mathbf{A}), \mathbf{G}=\hat{\mathbf{G}}(\mathbf{A}), \mathbf{A}=\hat{\mathbf{A}}(\mathbf{B})$ and $\mathbf{H}=\hat{\mathbf{H}}(\mathbf{A})$.

\section{A.4 Some identities of tensor analysis}

Let $\boldsymbol{u}$ and $\mathbf{A}$ be a smooth vector and tensor fields defined in $s$ and $v$ and $\mathbf{n}$ be the outward normal to $s$

$$
\int_{s} \boldsymbol{u} \times \mathbf{A n} d s=\int_{v}\left[\boldsymbol{u} \times \operatorname{div} \mathbf{A}+\mathcal{E}:\left(\operatorname{grad} \boldsymbol{u} \mathbf{A}^{\mathrm{T}}\right)\right] d v .
$$

Proof:

$$
\begin{aligned}
\int_{s} \boldsymbol{u} \times \mathbf{A n} d s & =\int_{v} \operatorname{div}(\boldsymbol{u} \times \mathbf{A}) d v=\int_{v}(\boldsymbol{u} \times \operatorname{div} \mathbf{A}+\operatorname{grad} \boldsymbol{u} \times \mathbf{A}) d v \\
& =\int_{v}\left[\boldsymbol{u} \times \operatorname{div} \mathbf{A}+\mathcal{E}:(\operatorname{grad} \boldsymbol{u}) \mathbf{A}^{\mathrm{T}}\right] d v,
\end{aligned}
$$

where the identity (A.1.41) is used for $\operatorname{grad} \boldsymbol{u} \times \mathbf{A}=\mathcal{E}:\left[(\operatorname{grad} \boldsymbol{u}) \mathbf{A}^{\mathrm{T}}\right]$.

Divergence of a vector product:

$$
\operatorname{div}(\boldsymbol{u} \times \boldsymbol{v})=\boldsymbol{v} \cdot \operatorname{curl} \boldsymbol{u}-\boldsymbol{u} \cdot \operatorname{curl} \boldsymbol{v}
$$

Gradient of a divergence:

$$
\begin{aligned}
\operatorname{grad} \operatorname{div} \boldsymbol{t} & =\operatorname{curl} \operatorname{curl} \boldsymbol{t}+\operatorname{div} \operatorname{grad} \boldsymbol{t}, \\
& =\operatorname{div}(\operatorname{grad} \boldsymbol{t})^{\mathrm{T}} .
\end{aligned}
$$

Divergence of a left mapping:

$$
\operatorname{div}(\boldsymbol{t} \mathbf{A})=\mathbf{A}: \operatorname{grad} \boldsymbol{t}+\boldsymbol{t} \cdot \operatorname{div} \mathbf{A} .
$$

Divergence of a product of a scalar-valued function and a vector-valued function:

$$
\operatorname{div}(g \boldsymbol{t})=\boldsymbol{t} \cdot \operatorname{grad} g+g \operatorname{div} \boldsymbol{t}
$$

Divergence of a product of a scalar-valued function and a tensor-valued function:

$$
\operatorname{div}(g \mathbf{A})=\mathbf{A} \operatorname{grad} g+g \operatorname{div} \mathbf{A}
$$


Divergence of a tensor product:

$$
\operatorname{div}(\boldsymbol{u} \otimes \boldsymbol{v})=(\operatorname{grad} \boldsymbol{u}) \boldsymbol{v}+\boldsymbol{u} \operatorname{div} \boldsymbol{v}
$$




\section{Appendix B}

\section{Continuum mechanics}

The short summary on continuum mechanics follows the lecture notes by Itskov [39], the textbook by Başar et al. [10], the lecture notes by Le [49], the textbook by Holzapfel [34], and the textbook by Lemaitre and Chaboche [50].

\section{B.1 Kinematics of deformation}

\section{Configuration and deformation}

A material body can be regarded as a set of material particles occupying some bounded regions of a three-dimensional Euclidean space $\mathbb{E}$.

As body moves, the region it occupies changes continuously. At time $t=t_{0}$ the body occupies the region $\mathcal{B}_{0} \subset \mathbb{E}$ called initial configuration. Let $\boldsymbol{X} \in \mathcal{B}_{0}$ denote the place of a particle of the body. A motion of the body is a one-parameter family of mappings $\phi: \mathcal{B}_{0} \rightarrow \mathcal{B}_{t} \subset \mathbb{E}$, where $\mathcal{B}_{t}$ is the region occupied by the body at time $t$ and called current configuration.

$$
\boldsymbol{x}=\boldsymbol{\varphi}(\boldsymbol{X}, t)=\boldsymbol{x}(\boldsymbol{X}, t), \quad \boldsymbol{X}=\boldsymbol{\varphi}^{-1}(\boldsymbol{x}, t)=\boldsymbol{X}(\boldsymbol{X}, t)
$$

where $\boldsymbol{x}$ corresponds to the place occupied by the same particle $\boldsymbol{X}$ in the current configuration $\mathcal{B}_{t}$. 


\section{Change of frame}

Let an event be characterized by the position of a particle at a certain time. Two different observers may describe the same event by the position $\boldsymbol{x}$ and $\boldsymbol{x}^{*}$ at times $\boldsymbol{t}$ and $\boldsymbol{t}^{*}$, respectively, according to their frame of reference. Assuming that both observers record the same differences in place and time for different events, the change of frame is described by the rigid motion and time shift

$$
\boldsymbol{x}^{*}=\boldsymbol{c}(t)+\mathbf{Q}(t) \boldsymbol{x}, \quad t^{*}=t-a, \quad \boldsymbol{c}(t) \in \mathbb{E}, \quad \mathbf{Q}(t) \in \text { Orth }
$$

For the initial configuration $\mathcal{B}_{0}$,

$$
\boldsymbol{x}^{*}\left(t_{0}\right)=\boldsymbol{c}\left(t_{0}\right)+\mathbf{Q}\left(t_{0}\right) \boldsymbol{X}=\boldsymbol{X} .
$$

The reference configuration is independent of the observer and thus $\boldsymbol{c}\left(t_{0}\right)=\boldsymbol{O}$ and $\mathbf{Q}\left(t_{0}\right)=\mathbf{I}$.

\section{Orthonormal basis, convective coordinates, tangent vectors}

The position of a particle in the reference and current configuration can be described with an orthonormal basis $\mathcal{E}=\left\{\boldsymbol{e}_{1}, \boldsymbol{e}_{2}, \boldsymbol{e}_{3}\right\}$

$$
\boldsymbol{X}=X^{i} \boldsymbol{e}_{i}, \quad \boldsymbol{x}=x^{i} \boldsymbol{e}_{i}
$$

where $X^{i}$ and $x^{i}$ are Lagrangian and Eulerian coordinates, respectively.

Introducing a system of convective coordinates $\theta_{i}, i=1,2,3$, the particle can be labelled by its coordinates

$$
\boldsymbol{X}=\hat{\boldsymbol{X}}\left(\theta_{1}, \theta_{2}, \theta_{3}\right), \quad \boldsymbol{x}=\hat{\boldsymbol{x}}\left(\theta_{1}, \theta_{2}, \theta_{3}, t\right)
$$

The tangent vectors to the coordinate lines $\theta^{i}, i=1,2,3$ in the reference and current configuration are defined by

$$
\boldsymbol{G}_{i}=\frac{\partial \boldsymbol{X}}{\partial \theta^{i}}=\frac{\partial X^{j}}{\partial \theta^{i}} \boldsymbol{e}_{j}=X_{, i}^{j} \boldsymbol{e}_{j}, \quad \boldsymbol{g}_{i}=\frac{\partial \boldsymbol{x}}{\partial \theta^{i}}=\frac{\partial x^{j}}{\partial \theta^{i}} \boldsymbol{e}_{j}=x_{, i}^{j} \boldsymbol{e}_{j}, \quad i=1,2,3 .
$$

Their dual bases are

$$
\boldsymbol{G}^{i}=\frac{\partial \theta^{i}}{\partial X^{j}} \boldsymbol{e}^{j}, \quad \boldsymbol{g}^{i}=\frac{\partial \theta^{i}}{\partial x^{j}} \boldsymbol{e}^{j}, \quad i=1,2,3 .
$$


The metric coefficients are

$$
G_{i j}=\boldsymbol{G}_{i} \cdot \boldsymbol{G}_{j}, \quad G^{i j}=\boldsymbol{G}^{i} \cdot \boldsymbol{G}^{j}, \quad g_{i j}=\boldsymbol{g}_{i} \cdot \boldsymbol{g}_{j}, \quad g^{i j}=\boldsymbol{g}_{i} \cdot \boldsymbol{g}_{j}
$$

\section{Deformation gradient}

The deformation gradient $\mathbf{F}$ is defined in terms of the bases (B.1.6) and (B.1.7)

$$
\mathbf{F}=\boldsymbol{g}_{i} \otimes \boldsymbol{G}^{i}, \quad \mathbf{F}^{-1}=\boldsymbol{G}_{i} \otimes \boldsymbol{g}^{i}, \quad \mathbf{F}^{\mathrm{T}}=\boldsymbol{G}^{i} \otimes \boldsymbol{g}_{i} \quad \mathbf{F}^{-\mathrm{T}}=\boldsymbol{g}^{i} \otimes \boldsymbol{G}_{i} .
$$

The bases transform according to

$$
\boldsymbol{g}_{i}=\mathbf{F} \boldsymbol{G}_{i}, \quad \boldsymbol{G}_{i}=\mathbf{F}^{-1} \boldsymbol{g}_{i}, \quad \boldsymbol{g}^{i}=\mathbf{F}^{-\mathrm{T}} \boldsymbol{G}^{i}=\boldsymbol{G}^{i} \mathbf{F}^{-1}, \quad \boldsymbol{G}^{i}=\mathbf{F}^{\mathrm{T}} \boldsymbol{g}^{i}
$$

Introducing the material and spatial gradients, $\operatorname{Grad}(\bullet)=\frac{\partial}{\partial X^{j}} \otimes \boldsymbol{e}^{j}$ and $\operatorname{grad}(\bullet)=\frac{\partial}{\partial x^{j}} \otimes \boldsymbol{e}^{j}$, the deformation gradient and its inverse can be represented by

$$
\begin{aligned}
\mathbf{F} & =\boldsymbol{g}_{i} \otimes \boldsymbol{G}^{i}=\frac{\partial x^{j}}{\partial \theta^{i}} \boldsymbol{e}_{j} \otimes \frac{\partial \theta^{i}}{\partial X^{k}} \boldsymbol{e}^{k}=\frac{\partial x^{j}}{\partial \theta^{i}} \frac{\partial \theta^{i}}{\partial X^{k}} \boldsymbol{e}_{j} \otimes \boldsymbol{e}^{k}=\frac{\partial x^{j}}{\partial X^{k}} \boldsymbol{e}_{j} \otimes \boldsymbol{e}^{k} \\
& =\frac{\partial \boldsymbol{x}}{\partial X^{k}} \otimes \boldsymbol{e}^{k}=\operatorname{Grad} \boldsymbol{x}, \\
\mathbf{F}^{-1} & =\boldsymbol{G}_{i} \otimes \boldsymbol{g}^{i}=\frac{\partial X^{j}}{\partial \theta^{i}} \boldsymbol{e}_{j} \otimes \frac{\partial \theta^{i}}{\partial x^{k}} \boldsymbol{e}^{k}=\frac{\partial X^{j}}{\partial \theta^{i}} \frac{\partial \theta^{i}}{\partial x^{k}} \boldsymbol{e}_{j} \otimes \boldsymbol{e}^{k}=\frac{\partial X^{j}}{\partial x^{k}} \boldsymbol{e}_{j} \otimes \boldsymbol{e}^{k} \\
& =\frac{\partial \boldsymbol{X}}{\partial x^{k}} \otimes \boldsymbol{e}^{k}=\operatorname{grad} \boldsymbol{X} .
\end{aligned}
$$

There again,

$$
\begin{aligned}
\mathbf{F} & =F_{\cdot j}^{i} \boldsymbol{e}_{i} \otimes \boldsymbol{e}^{j}=\operatorname{Grad} \boldsymbol{x}=\frac{\partial \boldsymbol{x}}{\partial \boldsymbol{X}}=\frac{\partial \boldsymbol{x}}{\partial X^{j}} \otimes \boldsymbol{e}^{j}=\frac{\partial x^{i}}{\partial X^{j}} \boldsymbol{e}_{i} \otimes \boldsymbol{e}^{j} \\
& =\frac{\partial \boldsymbol{x}}{\partial \theta^{k}} \frac{\partial \theta^{k}}{\partial X^{j}} \otimes \boldsymbol{e}^{j}=\boldsymbol{x}_{, k} \otimes \frac{\partial \theta^{k}}{\partial X^{j}} \boldsymbol{e}^{j}=\boldsymbol{g}_{k} \otimes \boldsymbol{G}^{k}, \\
\mathbf{F}^{-1} & =\left(F^{-1}\right)_{\cdot j}^{i} \boldsymbol{e}_{i} \otimes \boldsymbol{e}^{j}=\operatorname{grad} \boldsymbol{X}=\frac{\partial \boldsymbol{X}}{\partial \boldsymbol{x}}=\frac{\partial \boldsymbol{X}}{\partial x^{j}} \otimes \boldsymbol{e}^{j}=\frac{\partial X^{i}}{\partial x^{j}} \boldsymbol{e}_{i} \otimes \boldsymbol{e}^{j} \\
& =\frac{\partial \boldsymbol{X}}{\partial \theta^{k}} \frac{\partial \theta^{k}}{\partial x^{j}} \otimes \boldsymbol{e}^{j}=\frac{\partial \boldsymbol{X}}{\partial \theta^{k}} \otimes \frac{\partial \theta^{k}}{\partial x^{j}} \boldsymbol{e}^{j}=\boldsymbol{G}_{k} \otimes \boldsymbol{g}^{k} . \\
\mathbf{F F}^{-1} & =\frac{\partial \boldsymbol{x}}{\partial \boldsymbol{X}} \frac{\partial \boldsymbol{X}}{\partial \boldsymbol{x}}=\frac{\partial \boldsymbol{x}}{\partial \boldsymbol{x}}=\frac{\partial \boldsymbol{X}}{\partial \boldsymbol{X}}=\mathbf{I} \\
& =\left(\boldsymbol{g}_{i} \otimes \boldsymbol{G}^{i}\right)\left(\boldsymbol{G}_{j} \otimes \boldsymbol{g}^{j}\right)=\boldsymbol{G}^{i} \cdot \boldsymbol{G}_{j} \boldsymbol{g}_{i} \otimes \boldsymbol{g}^{j}=\delta_{j}^{i} \boldsymbol{g}_{i} \otimes \boldsymbol{g}^{j}=\boldsymbol{g}_{j} \otimes \boldsymbol{g}^{j}=\mathbf{I} .
\end{aligned}
$$




\section{Deformation of line, area and volume elements}

The vectorial line elements $d \boldsymbol{X}$ in the reference configuration $\mathcal{B}_{0}$ and $d \boldsymbol{x}$ in the current configuration $\mathcal{B}_{t}$ can be represented with respect to the convective coordinates by

$$
\begin{aligned}
& d \boldsymbol{X}=\frac{\partial \boldsymbol{X}}{\partial \theta^{i}} d \theta^{i}=\boldsymbol{X}_{, i} d \theta^{i}=\boldsymbol{G}_{i} d \theta^{i}, \\
& d \boldsymbol{x}=\frac{\partial \boldsymbol{x}}{\partial \theta^{i}} d \theta^{i}=\boldsymbol{x}_{, i} d \theta^{i}=\boldsymbol{g}_{i} d \theta^{i}=\mathbf{F} \boldsymbol{G}_{i} d \theta^{i}=\mathbf{F} d \boldsymbol{X} .
\end{aligned}
$$

The differential area element $d A$ with its unit normal $\boldsymbol{N}$ in the reference configuration $\mathcal{B}_{0}$ changes to the element $d a$ with unit normal $\boldsymbol{n}$ in the current configuration $\mathcal{B}_{t}$

$$
\boldsymbol{n} d a=(\operatorname{det} \mathbf{F}) \mathbf{F}^{-\mathrm{T}} \boldsymbol{N} d A=(\operatorname{adj} \mathbf{F})^{\mathrm{T}} \boldsymbol{N} d A=\operatorname{cof} \mathbf{F} \boldsymbol{N} d A
$$

which is known as Nanson's formula.

The volume change of an infinitesimal parallelepiped from $d V$ to $d v$ can be described by the determinant of the deformation gradient $\mathbf{F}$

$$
d v=J d V, \quad J=\operatorname{det} \mathbf{F} .
$$

\section{Strain and stretch measures}

The right and left Cauchy-Green strain tensors are defined by

$$
\begin{aligned}
& \mathbf{C}=\mathbf{F}^{\mathrm{T}} \mathbf{F}=\boldsymbol{G}^{i} \otimes \boldsymbol{g}_{i} \boldsymbol{g}_{j} \otimes \boldsymbol{G}^{j}=g_{i j} \boldsymbol{G}^{i} \otimes \boldsymbol{G}^{j}=C_{i j} \boldsymbol{G}^{i} \otimes \boldsymbol{G}^{j}, \\
& \mathbf{b}=\mathbf{F F}^{\mathrm{T}}=\boldsymbol{g}_{i} \otimes \boldsymbol{G}^{i} \boldsymbol{G}^{j} \otimes \boldsymbol{g}_{j}=G^{i j} \boldsymbol{g}_{i} \otimes \boldsymbol{g}_{j}=b^{i j} \boldsymbol{g}_{i} \otimes \boldsymbol{g}_{j} .
\end{aligned}
$$

As they are symmetric and positive definite, the spectral representation is possible:

$$
\mathbf{C}=\sum_{i=1}^{n_{e}} \Lambda_{i} \mathbf{P}_{i}, \quad \mathbf{b}=\sum_{i=1}^{n_{e}} \Lambda_{i} \mathbf{p}_{i}
$$

where $\Lambda_{i}$ denotes eigenvalues and the second-order tensors $\mathbf{P}_{i}$ and $\mathbf{p}_{i}$ are eigenprojections. The right and left stretch tensors are their square-roots

$$
\mathbf{U}=\mathbf{C}^{1 / 2}, \quad \mathbf{v}=\mathbf{b}^{1 / 2}
$$


According to the polar decomposition theorem, the deformation gradient $\mathbf{F}$ can be decomposed into a stretch tensor and a rotation tensor

$$
\mathbf{F}=\mathbf{R U}=\mathbf{v R}
$$

The right and left stretch tensor $\mathbf{U}$ and $\mathbf{v}$ are unique positive definite symmetric second-order tensors and the rotation tensor $\mathbf{R}$ is a proper orthogonal second-order tensor $\left(\mathbf{U}, \mathbf{v} \in \mathbf{S y m}^{+}\right.$ and $\mathbf{R} \in$ Orth).

Based on the stretch tensors generalized Lagrangian and Eulerian strain measures can be defined by

$$
\begin{aligned}
& \mathbf{E}^{(r)}= \begin{cases}\frac{1}{r}\left(\mathbf{U}^{r}-\mathbf{I}\right)=\frac{1}{r} \sum_{i=1}^{3}\left(\lambda_{i}^{r}-1\right) \boldsymbol{N}_{i} \otimes \boldsymbol{N}_{i}, & r \neq 0, \\
\ln \mathbf{U}=\sum_{i=1}^{3}\left(\ln \lambda_{i} \boldsymbol{N}_{i} \otimes \boldsymbol{N}_{i}\right), & r=0,\end{cases} \\
& \mathbf{e}^{(r)}= \begin{cases}\frac{1}{r}\left(\mathbf{v}^{r}-\mathbf{I}\right)=\frac{1}{r} \sum_{i=1}^{3}\left(\lambda_{i}^{r}-1\right) \boldsymbol{n}_{i} \otimes \boldsymbol{n}_{i}, & r \neq 0, \\
\ln \mathbf{v}=\sum_{i=1}^{3}\left(\ln \lambda_{i} \boldsymbol{n}_{i} \otimes \boldsymbol{n}_{i}\right), & r=0 .\end{cases}
\end{aligned}
$$

The Green-Lagrange strain tensor $\mathbf{E}$ is

$$
\mathbf{E}\left(=\mathbf{E}^{(2)}\right)=\frac{1}{2}\left(\mathbf{U}^{2}-\mathbf{I}\right)=\frac{1}{2}(\mathbf{C}-\mathbf{I}) .
$$

For the formulation of constitutive laws the principal invariants of the right and left CauchyGreen tensor are needed

$$
\mathrm{I}_{\mathbf{C}}\left(=\mathrm{I}_{\mathbf{b}}\right)=\operatorname{tr} \mathbf{C}, \quad \mathrm{II}_{\mathbf{C}}\left(=\mathrm{II}_{\mathbf{b}}\right)=\operatorname{tr}(\operatorname{cof} \mathbf{C}), \quad \mathrm{III}_{\mathbf{C}}\left(=\mathrm{III}_{\mathbf{b}}\right)=\operatorname{det} \mathbf{C} .
$$

\section{Velocity gradients}

The material and spatial time derivative denoted by $\frac{d}{d t}\{\bullet\}\left(\stackrel{\text { def }}{=} \mathrm{D}_{t}\{\bullet\}\right)$ and $\frac{\partial}{\partial t}\{\bullet\}$, respectively, 
are

$$
\frac{d}{d t}\{\bullet\}=\{\dot{\bullet}\}=\frac{\partial}{\partial t}\{\bullet\}+\boldsymbol{v} \cdot \operatorname{grad}\{\bullet\}
$$

Applying the material time derivative to the current position vector $\boldsymbol{x}$, the velocity can be obtained $\boldsymbol{v}=\dot{\boldsymbol{x}}$. Applying the material and spatial gradient operators as in (B.1.11), (B.1.12) to it, the material and spatial velocity gradients are defined by

$$
\begin{aligned}
\mathbf{L} & =\operatorname{Grad} \boldsymbol{v}=\frac{\partial \boldsymbol{v}}{\partial X^{j}} \otimes \boldsymbol{e}^{j}=\frac{\partial \boldsymbol{v}}{\partial \boldsymbol{X}}=\frac{\partial}{\partial \boldsymbol{X}}\left(\frac{\partial \boldsymbol{x}}{\partial t}\right)=\frac{\partial}{\partial t}\left(\frac{\partial \boldsymbol{x}}{\partial \boldsymbol{X}}\right)=\frac{\partial \mathbf{F}}{\partial t} \\
& =\dot{\mathbf{F}}=\left(\boldsymbol{g}_{i} \otimes \boldsymbol{G}^{i}\right)^{\cdot}=\dot{\boldsymbol{g}}_{i} \otimes \boldsymbol{G}^{i}+\boldsymbol{g}_{i} \otimes \dot{\boldsymbol{G}}^{i}=\dot{\boldsymbol{g}}_{i} \otimes \boldsymbol{G}^{i} \\
\mathbf{l} & =\operatorname{grad} \boldsymbol{v}=\frac{\partial \boldsymbol{v}}{\partial x^{j}} \otimes \boldsymbol{e}^{j}=\frac{\partial \boldsymbol{v}}{\partial \boldsymbol{x}}=\frac{\partial}{\partial \theta^{i}}\left(\frac{\partial \boldsymbol{x}}{\partial t}\right) \otimes \frac{\partial \theta^{i}}{\partial \boldsymbol{x}}=\frac{\partial}{\partial t}\left(\frac{\partial \boldsymbol{x}}{\partial \theta^{i}}\right) \otimes \frac{\partial \theta^{i}}{\partial \boldsymbol{x}} \\
& =\dot{\boldsymbol{g}}_{i} \otimes \boldsymbol{g}^{i}=\boldsymbol{v}_{, i} \otimes \boldsymbol{g}^{i}=\dot{\mathbf{F}} \boldsymbol{G}_{i} \otimes \boldsymbol{g}^{i}=\dot{\mathbf{F F}}^{-1} .
\end{aligned}
$$

The decomposition of spatial velocity gradient $\mathbf{l}$ is

$$
\mathbf{d}=\frac{1}{2}\left(\mathbf{l}+\mathbf{l}^{\mathrm{T}}\right), \quad \mathbf{w}=\frac{1}{2}\left(\mathbf{l}-\mathbf{l}^{\mathrm{T}}\right) .
$$

Here $\mathbf{d}$ is the symmetric rate deformation tensor and $\mathbf{w}$ is the skew-symmetric rate (the spin tensor).

Strain rate in Lagrangean description is defined by $\mathbf{D}$ :

$$
\mathbf{D}=\mathbf{F}^{\mathrm{T}} \mathbf{d} \mathbf{F}=\dot{\mathbf{E}}=\frac{1}{2} \dot{\mathbf{C}} .
$$

\section{B.2 Balance laws and Clausius-Duhem inequality}

\section{Stress}

Let us consider a body in the current configuration $\mathcal{B}_{t}$. We can imagine a smooth surface going through a point $P$ and separating the body into two parts and define a force $\Delta \boldsymbol{p}$ and a couple $\Delta \boldsymbol{m}$ resulting from the forces exerted by material on one side of the surface $\Delta a$ on the material 
on the other side. Supposing that the area $\Delta a$ converges to 0 with the point $P$ kept in it, the basic postulate of continuum mechanics is that the limit

$$
\lim _{\Delta a \rightarrow 0} \frac{\Delta \boldsymbol{p}}{\Delta a}=\boldsymbol{t}
$$

exists. The vector $\boldsymbol{t}$ is called Cauchy stress vector and depends on the surface only through the outward unit normal $\boldsymbol{n}$. According to the Cauchy's theorem the mapping $\boldsymbol{n} \rightarrow \boldsymbol{t}$ is linear provided $\boldsymbol{t}$ is a continuous function of the position vector $\boldsymbol{x}$ at $P$

$$
\boldsymbol{t}=\boldsymbol{\sigma n}
$$

where $\boldsymbol{\sigma}$ is the Cauchy stress tensor.

The other limit resulting from $\boldsymbol{m}$ is assumed to be zero vector

$$
\lim _{\Delta a \rightarrow 0} \frac{\Delta \boldsymbol{m}}{\Delta a}=\boldsymbol{O}
$$

The first Piola-Kirchhoff stress tensor can be introduced by

$$
\begin{aligned}
d \boldsymbol{p} & =\boldsymbol{t} d a=\boldsymbol{\sigma} \boldsymbol{n} d a=\boldsymbol{\sigma}(\operatorname{det} \mathbf{F}) \mathbf{F}^{-\mathrm{T}} \boldsymbol{N} d A=\mathbf{P} \boldsymbol{N} d A \\
\rightarrow \quad & \mathbf{P}=J \boldsymbol{\sigma} \mathbf{F}^{-\mathrm{T}}=\boldsymbol{\tau} \mathbf{F}^{-\mathrm{T}}
\end{aligned}
$$

where $\mathbf{P}$ is the first Piola-Kirchhoff stress tensor and $\boldsymbol{\tau}$ is the Kirchhoff stress tensor.

\section{Balance laws and Clausius-Duhem inequality}

- Balance of mass

$$
\mathrm{D}_{t} \rho+\rho \operatorname{div} \boldsymbol{v}=0, \quad \rho \operatorname{det}(\mathbf{F})=\rho_{0},
$$

where $\rho$ is the current mass density, $\rho_{0}$ is the referential mass density, and $\boldsymbol{v}$ is the velocity of material particle.

- Balance of linear momentum

$$
\rho \mathrm{D}_{t} \boldsymbol{v}=\operatorname{div} \boldsymbol{\sigma}+\rho \mathbf{b}, \quad \rho_{0} \ddot{\boldsymbol{x}}=\operatorname{Div} \mathbf{P}+\rho_{0} \mathbf{B}
$$

where $\boldsymbol{\sigma}$ is the Cauchy stress tensor, $\mathbf{P}$ is the first Piola-Kirchhoff stress tensor and $\mathbf{b}$ and $\mathbf{B}$ are body-force density in current an referential configuration, respectively. 
- Balance of angular momentum

$$
\boldsymbol{\sigma}=\boldsymbol{\sigma}^{\mathrm{T}}, \quad \mathbf{S}=\mathbf{S}^{\mathrm{T}}
$$

where $\mathbf{S}$ is the second Piola-Kirchhoff stress tensor.

- Balance of energy

$$
\rho \mathrm{D}_{t} e+\operatorname{div} \mathbf{q}=\boldsymbol{\sigma}: \operatorname{div} \boldsymbol{v}+\rho r, \quad \rho_{0} \dot{E}+\operatorname{Div} \mathbf{Q}=\rho_{0} R+\mathbf{S}: \mathbf{D},
$$

where $e$ is the internal energy density, $\mathbf{q}$ is the heat flux vector, and $r$ is the energy source per unit mass in current an referential configuration. $E, \mathbf{Q}$, and $R$ are counterparts in referential configuration.

- Clausius-Duhem inequality

$$
\begin{aligned}
& d=-\rho\left(\eta \mathrm{D}_{t} \theta+\mathrm{D}_{t} \psi\right)+\boldsymbol{\sigma}: \mathbf{d}-\mathbf{q} \cdot \frac{\operatorname{grad} \theta}{\theta} \geq 0 \\
& \mathcal{D}=-\rho_{0}(N \dot{\Theta}+\dot{\Psi})+\mathbf{S}: \mathbf{D}-\mathbf{Q} \cdot \frac{\operatorname{Grad} \Theta}{\Theta} \geq 0
\end{aligned}
$$

where $\eta$ and $N$ are the internal entropy density per unit mass, $\psi$ and $\Psi$ are free energy density defined by $\psi \stackrel{\text { def }}{=} e-\theta \eta$ and $\Psi \stackrel{\text { def }}{=} E-\Theta N$, respectively. The Clausius-Duhem inequality implies the thermodynamic admissibility of any process.

As heat flows in the opposite direction of the temperature gradient, the last term $-\mathbf{Q} \cdot \frac{\text { Grad } \Theta}{\Theta}$ (thermal dissipation) is greater than or equal to 0. Assuming that the thermal dissipation is negligible compared to mechanical one for slow processes, the inequality reduces to a more stringent form

$$
d=-\rho\left(\eta \mathrm{D}_{t} \theta+\mathrm{D}_{t} \psi\right)+\boldsymbol{\sigma}: \mathbf{d} \geq 0, \quad \mathcal{D}=-\rho_{0}(\dot{\Psi}+\dot{\Theta} N)+\mathbf{S}: \mathbf{D} \geq 0 .
$$

\section{Work-conjugate stress-strain pairs}

The pairs of stress and strain variables is called work-conjugate

$$
J \boldsymbol{\sigma}: \mathbf{d}=J \boldsymbol{\sigma}: \mathbf{l}=J \mathbf{F}^{-1} \boldsymbol{\sigma} \mathbf{F}^{-\mathrm{T}}: \dot{\mathbf{E}}^{(2)}=\mathbf{P}: \dot{\mathbf{F}}=\mathbf{S}: \frac{1}{2} \dot{\mathbf{C}}=\mathbf{S}: \dot{\mathbf{E}}=\mathbf{S}: \mathbf{D} .
$$




\section{B.3 Constitutive theory}

\section{General principles}

The properties of materials can be described by means of the specific stress-strain relations referred to as constitutive equations. The following general principles called Noll's axioms are the restrictions that all constitutive equations for all materials must satisfy.

\section{- Principle of determinism}

The stress in a particular point of the body at time $t$ is determined by the history of the motion of the body up to the time $t$.

- Principle of local action

With respect to the stress at a point $\boldsymbol{X}$, the motion of body points $\boldsymbol{Y}$ at a finite distance from $\boldsymbol{X}$ may be disregarded in comparsion with the motion at $\boldsymbol{X}$ itself and in its infinitesimal neighborhood.

- Principle of material objectivity

This principle is called also material frame indifference. According to it the constitutive equations of the material in consideration should have the same form in any coordinate system and remained unaffected by a supperposed rigid body motion.

\section{Simple elastic and hyperelastic materials}

A simple elastic material (Cauchy-elastic material) for which the current stress depends on only the current deformation state at time $t$, not on the history of deformation, has a constitutive equation based on the deformation gradient $\mathbf{F}$ :

$$
\boldsymbol{\sigma}=\mathbf{g}(\mathbf{F})=\check{\mathbf{g}}(\mathbf{F})
$$

Therefore, the work done by stresses in a simple elastic material depends on the path of deformation. The material has a non-conservative structure and the stress cannot be derived from a scalar potential function. 
On the contrary, a hyperelastic materials (Green elastic material) is a type of ideally elastic material whose stress-strain relationship derives from a strain energy density function $W$.

$W=\breve{W}(\mathbf{F})=\hat{W}(\mathbf{C})$ is postulated such that the response function $\boldsymbol{\sigma}(\mathbf{F})$ would be described by

$$
\boldsymbol{\sigma}(\mathbf{F})=J^{-1} \frac{\partial W}{\partial \mathbf{F}} \mathbf{F}^{\mathrm{T}}, \quad \mathbf{P}=\frac{\partial W}{\partial \mathbf{F}}, \quad \mathbf{S}=2 \frac{\partial W}{\partial \mathbf{C}}
$$

where $\boldsymbol{\sigma}$ is the Cauchy stress, $\mathbf{P}$ is the first Piola-Kirchhoff stress tensor, and $\mathbf{S}$ is the second Piola-Kirchhoff stress tensor, and $\mathbf{C}$ is the right Cauchy-Green deformation tensor.

In case of isothermal process $(\Theta=$ const) of perfectly elastic materials $(\mathcal{D}=0)$, the inequality (B.2.4) becomes

$$
\mathcal{D}=\mathbf{S}: \frac{1}{2} \dot{\mathbf{C}}-\rho_{0} \dot{\Psi}=2 \frac{\partial W}{\partial \mathbf{C}}: \frac{1}{2} \dot{\mathbf{C}}-\rho_{0} \dot{\Psi}=0 \quad \rightarrow \quad \dot{\Psi}=\frac{1}{\rho_{0}} \frac{\partial W}{\partial \mathbf{C}}: \dot{\mathbf{C}}
$$

where $W=\rho_{0} \Psi$.

\section{Materials with internal variables}

Introducing a set of parameters $\varsigma_{i}, i=1,2, \cdots n$ representing a material state that depends on the process history

$$
\begin{aligned}
\Psi & =\hat{\Psi}\left(\mathbf{C}, \varsigma_{i}\right), \quad \varsigma_{i}=1,2, \cdots n \\
\dot{\Psi} & =\frac{\partial \Psi}{\partial \mathbf{C}}: \dot{\mathbf{C}}+\sum_{i=1}^{n} \frac{\partial \Psi}{\partial \varsigma_{i}} \dot{\varsigma}_{i} .
\end{aligned}
$$

In case of isothermal process the dissipation is

$$
\begin{aligned}
\mathcal{D} & =\mathbf{S}: \frac{1}{2} \dot{\mathbf{C}}-\rho_{0} \dot{\Psi}=\mathbf{S}: \frac{1}{2} \dot{\mathbf{C}}-\rho_{0}\left(\frac{\partial \Psi}{\partial \mathbf{C}}: \dot{\mathbf{C}}+\sum_{i=1}^{n} \frac{\partial \Psi}{\partial \varsigma_{i}} \dot{\varsigma}_{i}\right) \\
& =\mathbf{S}: \frac{1}{2} \dot{\mathbf{C}}-\frac{\partial W}{\partial \mathbf{C}}: \dot{\mathbf{C}}-\rho_{0} \sum_{i=1}^{n} \frac{\partial \Psi}{\partial \varsigma_{i}} \dot{\zeta}_{i}=-\rho_{0} \sum_{i=1}^{n} \frac{\partial \Psi}{\partial \varsigma_{i}} \dot{\varsigma}_{i} \geq 0 .
\end{aligned}
$$

The current value of the internal variables is determined from a system of differential equations referred to as evolution equations:

$$
\dot{\varsigma}_{i}=f\left(\mathbf{C}, \varsigma_{i}\right), \quad i=1,2, \cdots n
$$


which are restricted by (B.3.6).

\section{Constrained materials}

For constrained materials, the constitutive equation is written by

$$
\mathbf{S}=2 \frac{\partial W}{\partial \mathbf{C}}+q \frac{\partial \gamma}{\partial \mathbf{C}}
$$

where $q$ is an arbitrary scalar and and $\gamma$ represents the constraint function defined by $\gamma(\mathbf{C})=0$. The stress rate takes the form

$$
\begin{aligned}
\dot{\mathbf{S}} & =\frac{\partial \mathbf{S}}{\partial \mathbf{C}}: \dot{\mathbf{C}}=2 \frac{\partial^{2} W}{\partial \mathbf{C} \partial \mathbf{C}}: \dot{\mathbf{C}}+\dot{q} \frac{\partial \gamma}{\partial \mathbf{C}}+q \frac{\partial^{2} \gamma}{\partial \mathbf{C} \partial \mathbf{C}}: \dot{\mathbf{C}}=\left(2 \frac{\partial^{2} W}{\partial \mathbf{C} \partial \mathbf{C}}+q \frac{\partial^{2} \gamma}{\partial \mathbf{C} \partial \mathbf{C}}\right): \dot{\mathbf{C}}+\dot{q} \frac{\partial \gamma}{\partial \mathbf{C}} \\
& =\mathbb{C}: \frac{1}{2} \dot{\mathbf{C}}+\dot{q} \frac{\partial \gamma}{\partial \mathbf{C}}
\end{aligned}
$$

where $\mathbb{C}=4 \frac{\partial^{2} W}{\partial \mathbf{C} \partial \mathbf{C}}+2 q \frac{\partial^{2} \gamma}{\partial \mathbf{C} \partial \mathbf{C}}$.

For incompressible materials expressed by $\operatorname{det} \mathbf{F}=1$ the constraint function can be given by

$$
\gamma(\mathbf{C})=(\operatorname{det} \mathbf{C})^{\frac{1}{3}}-1
$$

This leads the stress-strain relation

$$
\mathbf{S}=2 \frac{\partial W}{\partial \mathbf{C}}+\frac{q}{3} \mathbf{C}^{-1}
$$

\section{Nearly incompressible materials}

Multiplicative decomposition of the deformation gradient $\mathbf{F}$ into a volume preserving isochoric part $\overline{\mathbf{F}}$ and a volumetric part $J^{\frac{1}{3}} \mathbf{I}$ is

$$
\mathbf{F}=J^{\frac{1}{3}} \mathbf{I} \overline{\mathbf{F}}=J^{\frac{1}{3}} \overline{\mathbf{F}}
$$

In consideration of the objectivity condition, the free energy of a nearly-incompressible material can be expressed in the following form

$$
W=W_{\text {iso }}(\overline{\mathbf{C}})+W_{\text {vol }}(J), \quad \overline{\mathbf{C}}=\overline{\mathbf{F}}^{\mathrm{T}} \overline{\mathbf{F}}=\left(J^{-\frac{1}{3}} \mathbf{F}\right)^{\mathrm{T}} J^{-\frac{1}{3}} \mathbf{F}=J^{-\frac{2}{3}} \mathbf{C}
$$


where $W_{\text {vol }}(J)$ is the volumetric strain-energy function.

The second Piola-Kirchhoff tensor $\mathbf{S}$ is

$$
\mathbf{S}=2 \frac{\partial W}{\partial \mathbf{C}}=2 \frac{\partial W_{\text {iso }}}{\partial \mathbf{C}}+2 \frac{\partial W_{\text {vol }}}{\partial \mathbf{C}}=2 \frac{\partial W_{\text {iso }}}{\partial \overline{\mathbf{C}}}: \frac{\partial \overline{\mathbf{C}}}{\partial \mathbf{C}}+2 \frac{\partial W_{\text {vol }}}{\partial J} \frac{\partial J}{\partial \mathbf{C}} .
$$

With the following calculations and definitions

$$
\begin{aligned}
& 2 \frac{\partial W_{\text {iso }}}{\partial \overline{\mathbf{C}}} \stackrel{\text { def }}{=} \overline{\mathbf{S}}, \quad W_{\text {vol }}^{\prime} \stackrel{\text { def }}{=} \frac{\partial W_{\text {vol }}}{\partial J}, \\
& \frac{\partial \overline{\mathbf{C}}}{\partial \mathbf{C}}=\frac{\partial}{\partial \mathbf{C}}\left(J^{-\frac{2}{3}} \mathbf{C}\right)=\mathbf{C} \odot \frac{\partial J^{-\frac{2}{3}}}{\partial \mathbf{C}}+J^{-\frac{2}{3}} \frac{\partial \mathbf{C}}{\partial \mathbf{C}}=\mathbf{C} \odot \frac{\partial J^{-\frac{2}{3}}}{\partial J} \frac{\partial J}{\partial \mathbf{C}}+J^{-\frac{2}{3}} \mathbb{I} \\
& =\mathbf{C} \odot\left(-\frac{2}{3}\right) J^{-\frac{5}{3}} \frac{J}{2} \mathbf{C}^{-\mathrm{T}}+J^{-\frac{2}{3}} \mathbb{I}=J^{-\frac{2}{3}}\left(-\frac{1}{3} \mathbf{C} \odot \mathbf{C}^{-\mathrm{T}}+\mathbb{I}\right) \stackrel{\text { def }}{=} J^{-\frac{2}{3}} \mathbb{P}^{\mathrm{T}}, \\
& \frac{\partial J^{2}}{\partial \mathbf{C}}=\frac{\partial \operatorname{det} \mathbf{C}}{\partial \mathbf{C}}=\frac{\partial J^{2}}{\partial J} \frac{\partial J}{\partial \mathbf{C}} \rightarrow \operatorname{det} \mathbf{C C}^{-\mathrm{T}}=2 J \frac{\partial J}{\partial \mathbf{C}} \rightarrow \frac{\partial J}{\partial \mathbf{C}}=\frac{J}{2} \mathbf{C}^{-\mathrm{T}},
\end{aligned}
$$

the stress can be given in the from

$$
\mathbf{S}=\overline{\mathbf{S}}: J^{-\frac{2}{3}} \mathbb{P}^{\mathrm{T}}+2 W_{\text {vol }}^{\prime} \frac{J}{2} \mathbf{C}^{-\mathrm{T}}=J^{-\frac{2}{3}} \mathbb{P}: \overline{\mathbf{S}}+J W_{\mathrm{vol}}^{\prime} \mathbf{C}^{-\mathrm{T}} \stackrel{\text { def }}{=} \mathbf{S}_{\mathrm{iso}}+\mathbf{S}_{\mathrm{vol}} .
$$

The fourth order-tensorP is called the projection tensor and reads

$$
\mathbb{P}=\mathbb{P}^{\mathrm{TT}}=\left(-\frac{1}{3} \mathbf{C} \odot \mathbf{C}^{-\mathrm{T}}+\mathbb{I}\right)^{\mathrm{T}}=-\frac{1}{3} \mathbf{C}^{-\mathrm{T}} \odot \mathbf{C}+\mathbb{I}^{\mathrm{T}}=-\frac{1}{3} \mathbf{C}^{-1} \odot \mathbf{C}+\mathbb{I} .
$$

$\mathbf{S}_{\text {iso }}$ is the stress due to the isochoric deformations and $\mathbf{S}_{\mathrm{vol}}$ stress due to the volumetric deformations.

\section{Stress rates and elasticity tensor}

For the hyperelastic materials the material time derivative of the second Piola-Kirchhoff stress tensor gives

$$
\dot{\mathbf{S}}=2 \frac{\partial \mathbf{S}}{\partial \mathbf{C}}: \frac{1}{2} \dot{\mathbf{C}}=2 \frac{\partial}{\partial \mathbf{C}}\left(2 \frac{\partial W}{\partial \mathbf{C}}\right): \frac{1}{2} \dot{\mathbf{C}}=4 \frac{\partial^{2} W}{\partial \mathbf{C} \partial \mathbf{C}}: \frac{1}{2} \dot{\mathbf{C}}=\mathbb{C}: \frac{1}{2} \dot{\mathbf{C}},
$$

where $\mathbb{C}\left(=\mathbb{C}^{\mathrm{T}}\right)=2 \frac{\partial \mathbf{S}}{\partial \mathbf{C}}=4 \frac{\partial^{2} W}{\partial \mathbf{C} \partial \mathbf{C}}$ is super-symmetric material tangent tensor of fourth order. The material time derivative of the first Piola-Kirchhoff stress is

$$
\dot{\mathbf{P}}=\frac{\partial \mathbf{P}}{\partial \mathbf{F}}: \dot{\mathbf{F}}=\frac{\partial}{\partial \mathbf{F}}\left(\frac{\partial W}{\partial \mathbf{F}}\right): \dot{\mathbf{F}}=\frac{\partial^{2} W}{\partial \mathbf{F} \partial \mathbf{F}}: \dot{\mathbf{F}}=\mathbb{A}: \dot{\mathbf{F}}
$$


where $\mathbb{A}=\frac{\partial \mathbf{P}}{\partial \mathbf{F}}=\frac{\partial^{2} W}{\partial \mathbf{F} \partial \mathbf{F}}$ denotes as elasticity tensor.

The Oldroyd and Jaumann rates of Kirchhoff stress tensor $\boldsymbol{\tau}\left(\stackrel{\text { def }}{=} \mathbf{P F}^{\mathrm{T}}\right)$ are given by

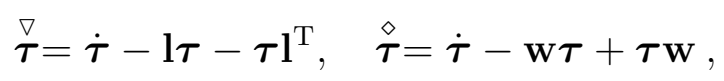

respectively. They have the relations $\stackrel{\diamond}{\boldsymbol{\tau}}=\stackrel{\nabla}{\boldsymbol{\tau}}+\mathbf{d} \boldsymbol{\tau}+\boldsymbol{\tau} \mathbf{d}$.

The Oldroyd rate of $\boldsymbol{\tau}$ can be represented as a mapping of the symmetric rate deformation tensor $\mathbf{d}$

$$
\stackrel{\nabla}{\boldsymbol{\tau}}=\left[\left(\mathbf{F} \otimes \mathbf{F}^{\mathrm{T}}\right): \mathbb{C}:\left(\mathbf{F}^{\mathrm{T}} \otimes \mathbf{F}\right)\right]: \mathbf{d}=\mathbb{C}: \mathbf{d}
$$

where $\mathbb{C}$ denotes the spatial tangent tensor.

For incompressible constrained materials, (B.3.8), (B.3.9) and (B.3.10), we derive the constrained function $\gamma$ with respect to $\mathbf{C}$

$$
\begin{aligned}
\frac{\partial \gamma}{\partial \mathbf{C}} & =\frac{\partial}{\partial \mathbf{C}}\left[(\operatorname{det} \mathbf{C})^{\frac{1}{3}}-1\right]=\left[(\operatorname{det} \mathbf{C})^{\frac{1}{3}}\right]_{, \mathbf{C}}=\frac{\partial(\operatorname{det} \mathbf{C})^{\frac{1}{3}}}{\partial(\operatorname{det} \mathbf{C})} \frac{\partial(\operatorname{det} \mathbf{C})}{\partial \mathbf{C}} \\
& =\frac{1}{3}(\operatorname{det} \mathbf{C})^{-\frac{2}{3}} \operatorname{det} \mathbf{C} \mathbf{C}^{-\mathrm{T}}=\frac{1}{3}(\operatorname{det} \mathbf{C})^{\frac{1}{3}} \mathbf{C}^{-\mathrm{T}}=\frac{1}{3}(\operatorname{det} \mathbf{C})^{\frac{1}{3}} \mathbf{C}^{-1}, \\
\frac{\partial^{2} \gamma}{\partial \mathbf{C} \partial \mathbf{C}} & =\frac{\partial}{\partial \mathbf{C}}\left(\frac{\partial \gamma}{\partial \mathbf{C}}\right)=\frac{\partial}{\partial \mathbf{C}}\left[\frac{1}{3}(\operatorname{det} \mathbf{C})^{\frac{1}{3}} \mathbf{C}^{-1}\right] \\
& =\frac{1}{3}\left[\mathbf{C}^{-1} \odot\left((\operatorname{det} \mathbf{C})^{\frac{1}{3}}\right)_{, \mathbf{C}}+(\operatorname{det} \mathbf{C})^{\frac{1}{3}}\left(\mathbf{C}^{-1}\right)_{, \mathbf{C}}\right] \\
& =\frac{1}{3}\left[\frac{1}{3}(\operatorname{det} \mathbf{C})^{\frac{1}{3}} \mathbf{C}^{-1} \odot \mathbf{C}^{-1}-(\operatorname{det} \mathbf{C})^{\frac{1}{3}} \mathbf{C}^{-1} \otimes \mathbf{C}^{-1}\right] \\
& =\frac{1}{3}(\operatorname{det} \mathbf{C})^{\frac{1}{3}}\left[\frac{1}{3} \mathbf{C}^{-1} \odot \mathbf{C}^{-1}-\mathbf{C}^{-1} \otimes \mathbf{C}^{-1}\right] .
\end{aligned}
$$

Then the material tangent tensor $\mathbb{C}$ for the incompressible constrained materials in (B.3.9) can be given by

$$
\mathbb{C}=4 \frac{\partial^{2} W}{\partial \mathbf{C} \partial \mathbf{C}}+2 q \frac{\partial^{2} \gamma}{\partial \mathbf{C} \partial \mathbf{C}}=4 \frac{\partial^{2} W}{\partial \mathbf{C} \partial \mathbf{C}}+\frac{2 q}{3}(\operatorname{det} \mathbf{C})^{\frac{1}{3}}\left[\frac{1}{3} \mathbf{C}^{-1} \odot \mathbf{C}^{-1}-\mathbf{C}^{-1} \otimes \mathbf{C}^{-1}\right] .
$$

For nearly-incompressible materials (B.3.15) the material tangent tensor $\mathbb{C}$ is introduced as

$$
\mathbb{C}_{\text {def }}^{=} \mathbb{C}_{\text {iso }}+\mathbb{C}_{\text {vol }} \text {. }
$$


The isochoric material tangent tensor $\mathbb{C}_{\text {iso }}$ reads

$$
\begin{aligned}
\mathbb{C}_{\text {iso }}= & 2 \frac{\partial \mathbf{S}_{\text {iso }}}{\partial \mathbf{C}}=2\left(J^{-\frac{2}{3}} \mathbb{P}: \overline{\mathbf{S}}\right)_{, \mathbf{C}}=2(\mathbb{P}: \overline{\mathbf{S}}) \odot\left(J^{-\frac{2}{3}}\right)_{, \mathbf{C}}+2 J^{-\frac{2}{3}}(\mathbb{P}: \overline{\mathbf{S}})_{, \mathbf{C}} \\
= & 2(\mathbb{P}: \overline{\mathbf{S}}) \odot\left(-\frac{1}{3} J^{-\frac{2}{3}} \mathbf{C}^{-\mathrm{T}}\right)+2 J^{-\frac{2}{3}}(\mathbb{P}: \overline{\mathbf{S}})_{, \mathbf{C}} \\
= & -\frac{2}{3} J^{-\frac{2}{3}}(\mathbb{P}: \overline{\mathbf{S}}) \odot \mathbf{C}^{-\mathrm{T}}+2 J^{-\frac{2}{3}}(\mathbb{P}: \overline{\mathbf{S}})_{, \mathbf{C}} \\
= & -\frac{2}{3} J^{-\frac{2}{3}}[-\frac{1}{3} \mathbf{C}^{-1}(\mathbf{C}: \overline{\mathbf{S}})+\overline{\mathbf{S}} \overbrace{\mathbf{C}^{-\mathrm{T}}} \\
& +2 J^{-\frac{2}{3}}\left[-\frac{1}{3} \mathbf{C}^{-1} \odot\left(\overline{\mathbf{S}}+\mathbf{C}: \overline{\mathbf{S}}_{, \mathbf{C}}\right)+\frac{1}{3}(\mathbf{C}: \overline{\mathbf{S}})\left(\mathbf{C}^{-1} \otimes \mathbf{C}^{-1}\right)+\overline{\mathbf{S}}_{, \mathbf{C}}\right] \\
= & -\frac{2}{3} J^{-\frac{2}{3}}\left[-\frac{1}{3}(\mathbf{C}: \overline{\mathbf{S}}) \mathbf{C}^{-1} \odot \mathbf{C}^{-1}+\overline{\mathbf{S}} \odot \mathbf{C}^{-1}\right] \\
& -\frac{2}{3} J^{-\frac{2}{3}}\left[\mathbf{C}^{-1} \odot\left(\overline{\mathbf{S}}+\mathbf{C}: \overline{\mathbf{S}}_{, \mathbf{C}}\right)-(\mathbf{C}: \overline{\mathbf{S}})\left(\mathbf{C}^{-1} \otimes \mathbf{C}^{-1}\right)-3 \overline{\mathbf{S}}_{, \mathbf{C}}\right] \\
= & \frac{2}{3} J^{-\frac{2}{3}}(\mathbf{C}: \overline{\mathbf{S}})\left(\frac{1}{3} \mathbf{C}^{-1} \odot \mathbf{C}^{-1}+\mathbf{C}^{-1} \otimes \mathbf{C}^{-1}\right)-\frac{2}{3} J^{-\frac{2}{3}}\left(\overline{\mathbf{S}} \odot \mathbf{C}^{-1}+\mathbf{C}^{-1} \odot \overline{\mathbf{S}}\right) \\
& -\frac{2}{3} J^{-\frac{2}{3}} \mathbf{C}^{-1} \odot\left(\mathbf{C}: \overline{\mathbf{S}}_{, \mathbf{C}}\right)+2 J^{-\frac{2}{3}} \overline{\mathbf{S}}_{, \mathbf{C}},
\end{aligned}
$$

where the derivation of $J^{-\frac{2}{3}}$ with respect to $\mathbf{C}$ is

$$
\frac{\partial J^{-\frac{2}{3}}}{\partial \mathbf{C}}=\frac{\partial J^{-\frac{2}{3}}}{\partial J} \frac{\partial J}{\partial \mathbf{C}}=-\frac{2}{3} J^{-\frac{5}{3}} \frac{J}{2} \mathbf{C}^{-\mathrm{T}}=-\frac{1}{3} J^{-\frac{2}{3}} \mathbf{C}^{-\mathrm{T}}
$$

and

$$
\mathbb{P}: \overline{\mathbf{S}}=\left(-\frac{1}{3} \mathbf{C}^{-1} \odot \mathbf{C}+\mathbb{I}\right): \overline{\mathbf{S}}=-\frac{1}{3}\left(\mathbf{C}^{-1} \odot \mathbf{C}\right): \overline{\mathbf{S}}+\mathbb{I}: \overline{\mathbf{S}}=-\frac{1}{3} \mathbf{C}^{-1}(\mathbf{C}: \overline{\mathbf{S}})+\overline{\mathbf{S}}
$$

its derivation with respect to $\mathbf{C}$ is

$$
\begin{aligned}
(\mathbb{P}: \overline{\mathbf{S}})_{, \mathbf{C}} & =\left[-\frac{1}{3} \mathbf{C}^{-1}(\mathbf{C}: \overline{\mathbf{S}})+\overline{\mathbf{S}}\right]_{, \mathbf{C}}=-\frac{1}{3} \mathbf{C}^{-1} \odot(\mathbf{C}: \overline{\mathbf{S}})_{, \mathbf{C}}-\frac{1}{3}\left(\mathbf{C}: \overline{\mathbf{S}}^{-1}\left(\mathbf{C}^{-1}\right)_{, \mathbf{C}}+\overline{\mathbf{S}}_{, \mathbf{C}}\right. \\
& =-\frac{1}{3} \mathbf{C}^{-1} \odot\left(\overline{\mathbf{S}}: \mathbf{C}_{, \mathbf{C}}+\mathbf{C}: \overline{\mathbf{S}}_{, \mathbf{C}}\right)-\frac{1}{3}(\mathbf{C}: \overline{\mathbf{S}})\left(-\mathbf{C}^{-1} \otimes \mathbf{C}^{-1}\right)+\overline{\mathbf{S}}_{, \mathbf{C}} \\
& =-\frac{1}{3} \mathbf{C}^{-1} \odot\left(\overline{\mathbf{S}}: \mathbb{I}+\mathbf{C}: \overline{\mathbf{S}}_{, \mathbf{C}}\right)+\frac{1}{3}(\mathbf{C}: \overline{\mathbf{S}})\left(\mathbf{C}^{-1} \otimes \mathbf{C}^{-1}\right)+\overline{\mathbf{S}}_{, \mathbf{C}} \\
& =-\frac{1}{3} \mathbf{C}^{-1} \odot\left(\overline{\mathbf{S}}+\mathbf{C}: \overline{\mathbf{S}}_{, \mathbf{C}}\right)+\frac{1}{3}(\mathbf{C}: \overline{\mathbf{S}})\left(\mathbf{C}^{-1} \otimes \mathbf{C}^{-1}\right)+\overline{\mathbf{S}}_{, \mathbf{C}} .
\end{aligned}
$$


The volumetric material tensor $\mathbb{C}_{\mathrm{vol}}$ is obtained

$$
\begin{aligned}
\mathbb{C}_{\mathrm{vol}} & =2 \frac{\partial \mathbf{S}_{\mathrm{vol}}}{\partial \mathbf{C}}=2\left(J W_{\mathrm{vol}}^{\prime} \mathbf{C}^{-1}\right)_{, \mathbf{C}}=2\left[\mathbf{C}^{-1} \odot\left(J W_{\mathrm{vol}}^{\prime}\right)_{, \mathbf{C}}+J W_{\mathrm{vol}}^{\prime}\left(\mathbf{C}^{-1}\right)_{, \mathbf{C}}\right] \\
& =2\left[\mathbf{C}^{-1} \odot\left(J_{, \mathbf{C}} W_{\mathrm{vol}}^{\prime}+J W_{\mathrm{vol}, \mathbf{C}}^{\prime}\right)+J W_{\mathrm{vol}}^{\prime}\left(\mathbf{C}^{-1}\right)_{, \mathbf{C}}\right] \\
& =2\left[\mathbf{C}^{-1} \odot\left(J_{, \mathbf{C}} W_{\mathrm{vol}}^{\prime}+J W_{\mathrm{vol}}^{\prime \prime} J_{, \mathbf{C}}\right)-J W_{\mathrm{vol}}^{\prime}\left(\mathbf{C}^{-1} \otimes \mathbf{C}^{-1}\right)\right] \\
& =2\left[\frac{J}{2} \mathbf{C}^{-1} \odot \mathbf{C}^{-\mathrm{T}}\left(W_{\mathrm{vol}}^{\prime}+J W_{\mathrm{vol}}^{\prime \prime}\right)-J W_{\mathrm{vol}}^{\prime}\left(\mathbf{C}^{-1} \otimes \mathbf{C}^{-1}\right)\right] \\
& =J\left(W_{\mathrm{vol}}^{\prime}+J W_{\mathrm{vol}}^{\prime \prime}\right) \mathbf{C}^{-1} \odot \mathbf{C}^{-1}-2 J W_{\mathrm{vol}}^{\prime}\left(\mathbf{C}^{-1} \otimes \mathbf{C}^{-1}\right) .
\end{aligned}
$$


APPENDIX B. CONTINUUM MECHANICS 


\section{Appendix C}

\section{Appendix}

\section{C.1 Balance of mass in the current configuartion}

The spatial velocity of species $i(i \neq \mathrm{S})$ is defined as

$$
\boldsymbol{v}^{i}=\frac{\boldsymbol{m}^{i}}{\rho^{i}}, \quad i \neq \mathrm{S}
$$

where $\rho^{i}$ and $\boldsymbol{m}^{i}$ are mass density and mass flux of species $i$ in current configuration, respectively.

We can show that the material velocity and the spatial velocity are the same:

$$
(\mathrm{C} .1 .7) \quad \rightarrow \quad \mathbf{F} \boldsymbol{M}^{i}=J \boldsymbol{m}^{i} \stackrel{(\mathrm{C} .1 .5)}{=} \frac{\rho_{0}^{i}}{\rho^{i}} \boldsymbol{m}^{i} \quad \rightarrow \quad \boldsymbol{V}^{i}=\frac{1}{\rho_{0}^{i}} \mathbf{F} \boldsymbol{M}^{i}=\frac{\boldsymbol{m}^{i}}{\rho^{i}}=\boldsymbol{v}^{i} .
$$

In the current configuration, $\mathcal{B}$ occupies the open region $\Omega_{t} \subset \mathbb{R}^{3}$ whose boundary is $\partial \Omega_{t}$ and its outward normal vector is $\boldsymbol{n}$. The density and the source of any species $(i=\mathrm{S}, \mathrm{F}, \mathrm{N}, \mathrm{B})$ are, respectively, denoted by $\rho^{i}(\boldsymbol{x}, t)$ and $\pi^{i}(\boldsymbol{x}, t)$. The mass flux is denoted by $\boldsymbol{m}^{i}(\boldsymbol{x}, t)$.

The global form of the balance of mass in the current configuration is

$$
\frac{d}{d t} \int_{m^{i}} d m^{i}=\frac{d}{d t} \int_{\Omega_{t}} \rho^{i} d v=\int_{\Omega_{t}} \pi^{i} d v-\int_{\partial \Omega_{t}} \boldsymbol{m}^{i} \cdot d \boldsymbol{a}
$$

By using the following Reynolds transport theorem for a general balanced quantity $\{\bullet\}^{i}$,

$$
\frac{d}{d t} \int_{\Omega_{t}}\{\bullet\}^{i} d v=\int_{\Omega_{t}}\left[\frac{\partial}{\partial t}\{\bullet\}^{i}+\operatorname{div}\left(\{\bullet\}^{i} \otimes \boldsymbol{v}\right)\right] d v,
$$

the left-hand side can be expressed as

$$
\frac{d}{d t} \int_{\Omega_{t}} \rho^{i} d v=\int_{\Omega_{t}}\left[\frac{\partial \rho^{i}}{\partial t}+\operatorname{div}\left(\rho^{i} \boldsymbol{v}\right)\right] d v .
$$


By using the divergence theorem, the right-hand side becomes

$$
\int_{\Omega_{t}} \pi^{i} d v-\int_{\partial \Omega_{t}} \boldsymbol{m}^{i} \cdot d \boldsymbol{a}=\int_{\Omega_{t}} \pi^{i} d v-\int_{\Omega_{t}} \operatorname{div} \boldsymbol{m}^{i} d v=\int_{\Omega_{t}}\left(\pi^{i}-\operatorname{div} \boldsymbol{m}^{i}\right) d v .
$$

By equating the both expressions

$$
\rightarrow \quad \frac{\partial \rho^{i}}{\partial t}+\operatorname{div}\left(\rho^{i} \boldsymbol{v}\right)=\pi^{i}-\operatorname{div} \boldsymbol{m}^{i}, \quad \forall i=\mathrm{S}, \mathrm{F}, \mathrm{N}, \mathrm{B} . \quad \square
$$

It can be also derived from the following Piola-transforms:

$$
\begin{gathered}
\int_{\Omega_{0}} \rho_{0}^{i} d V=\int_{\Omega_{t}} \rho^{i} d v=\int_{\Omega_{0}} \rho^{i} J d V \quad \rightarrow \quad \rho_{0}^{i}=J \rho^{i} \\
\int_{\Omega_{0}} \Pi^{i} d V=\int_{\Omega_{t}} \pi^{i} d v=\int_{\Omega_{0}} \pi^{i} J d V \rightarrow \Pi^{i}=J \pi^{i} \\
\int_{\partial \Omega_{0}} \boldsymbol{M}^{i} \cdot d \boldsymbol{A}=\int_{\partial \Omega_{t}} \boldsymbol{m}^{i} \cdot d \boldsymbol{a}=\int_{\partial \Omega_{0}} \boldsymbol{m}^{i} \cdot J \mathbf{F}^{-T} d \boldsymbol{A}=\int J \mathbf{F}^{-1} \boldsymbol{m}^{i} \cdot d \boldsymbol{A} \\
\rightarrow \quad \boldsymbol{M}^{i}=J \mathbf{F}^{-1} \boldsymbol{m}^{i}
\end{gathered}
$$

where $d v=J d V$ and $J=\operatorname{det} \mathbf{F}$.

Applying these transforms to Eq. (3.2.5), we obtain

$$
\frac{d\left(J \rho^{i}\right)}{d t}=J \pi^{i}-\operatorname{Div}\left(J \mathbf{F}^{-1} \boldsymbol{m}^{i}\right)
$$

The left-hand side of Eq. (C.1.8) reads

$$
\begin{aligned}
\text { LHS } & =\frac{d J}{d t} \rho^{i}+J \frac{d \rho^{i}}{d t}=J \operatorname{div} \boldsymbol{v} \rho^{i}+J \frac{d \rho^{i}}{d t}=J\left(\operatorname{div} \boldsymbol{v} \rho^{i}+\frac{d \rho^{i}}{d t}\right) \\
& =J\left(\operatorname{div} \boldsymbol{v} \rho^{i}+\frac{\partial \rho^{i}}{\partial t}+\operatorname{grad} \rho^{i} \cdot \boldsymbol{v}\right)=J\left(\frac{\partial \rho^{i}}{\partial t}+\rho^{i} \operatorname{div} \boldsymbol{v}+\boldsymbol{v} \cdot \operatorname{grad} \rho^{i}\right) \\
& =J\left[\frac{\partial \rho^{i}}{\partial t}+\operatorname{div}\left(\rho^{i} \boldsymbol{v}\right)\right] .
\end{aligned}
$$


The second term of the right-hand sides is

$$
\begin{aligned}
\operatorname{Div}\left(J \mathbf{F}^{-1} \boldsymbol{m}^{i}\right) & =\left(J \mathbf{F}^{-1}\right)^{\mathrm{T}}: \operatorname{Grad} \boldsymbol{m}^{i}+\boldsymbol{m}^{i} \cdot \operatorname{Div}\left(J \mathbf{F}^{-1}\right)^{\mathrm{T}} \\
& =J \mathbf{F}^{-T}: \operatorname{Grad} \boldsymbol{m}^{i}+\boldsymbol{m}^{i} \cdot \operatorname{Div}\left(J\left(\mathbf{F}^{\mathrm{T}}\right)^{-1}\right) \\
& =J\left(\frac{\partial \boldsymbol{X}}{\partial \boldsymbol{x}}\right)^{\mathrm{T}}: \frac{\partial \boldsymbol{m}^{i}}{\partial \boldsymbol{X}}+\boldsymbol{m}^{i} \cdot \operatorname{Div}\left(J \frac{1}{\operatorname{det} \mathbf{F}^{\mathrm{T}}}\left[\operatorname{adj}\left(\mathbf{F}^{\mathrm{T}}\right)\right]^{\mathrm{T}}\right) \\
& =J \frac{\partial m_{j}^{i}}{\partial x_{j}}+\boldsymbol{m}^{i} \cdot \operatorname{Div}\left(J \frac{1}{\operatorname{det} \mathbf{F}} \operatorname{adj} \mathbf{F}\right) \\
& =J \operatorname{div} \boldsymbol{m}^{i}+\boldsymbol{m}^{i} \cdot \operatorname{Div}(\operatorname{adj} \mathbf{F}) \\
& =J \operatorname{div} \boldsymbol{m}^{i},
\end{aligned}
$$

where

$$
\begin{aligned}
& {[\operatorname{Div}(\operatorname{adj} \mathbf{F})]_{j}=(\operatorname{adj} \mathbf{F})_{j A, A},} \\
& \mathbf{F}=\left[\begin{array}{lll}
F_{11} & F_{12} & F_{13} \\
F_{21} & F_{22} & F_{23} \\
F_{31} & F_{32} & F_{33}
\end{array}\right], \\
& \operatorname{adj} \mathbf{F}=\left[\begin{array}{lll}
F_{22} F_{33}-F_{23} F_{32} & F_{23} F_{31}-F_{21} F_{33} & F_{21} F_{32}-F_{31} F_{22} \\
F_{13} F_{32}-F_{12} F_{33} & F_{11} F_{33}-F_{13} F_{31} & F_{12} F_{31}-F_{11} F_{32} \\
F_{12} F_{23}-F_{13} F_{22} & F_{21} F_{13}-F_{11} F_{23} & F_{11} F_{22}-F_{12} F_{21}
\end{array}\right] .
\end{aligned}
$$

In case of $j=1$, Eq. (C.1.10) becomes

$$
\begin{aligned}
(\operatorname{adj} \mathbf{F})_{1 A, A} & =(\operatorname{adj} \mathbf{F})_{11,1}+(\operatorname{adj} \mathbf{F})_{12,2}+(\operatorname{adj} \mathbf{F})_{13,3} \\
= & \left(F_{22} F_{33}-F_{23} F_{32}\right)_{, 1}+\left(F_{23} F_{31}-F_{21} F_{33}\right)_{, 2}+\left(F_{21} F_{32}-F_{31} F_{22}\right)_{, 3} \\
= & F_{22,1} F_{33}+F_{22} F_{33,1}-F_{23,1} F_{32}-F_{23} F_{32,1} \\
& +F_{23,2} F_{31}+F_{23} F_{31,2}-F_{21,2} F_{33}-F_{21} F_{33,2} \\
& +F_{21,3} F_{32}+F_{21} F_{32,3}-F_{31,3} F_{22}-F_{31} F_{22,3} \\
= & F_{21,2} F_{33}+F_{22} F_{31,3}-F_{23,1} F_{32}-F_{23} F_{32,1} \\
& +F_{22,3} F_{31}+F_{23} F_{32,1}-F_{21,2} F_{33}-F_{21} F_{33,2} \\
& +F_{23,1} F_{32}+F_{21} F_{33,2}-F_{31,3} F_{22}-F_{31} F_{22,3} \\
= & 0
\end{aligned}
$$


where $F_{j A, B}=\left(x_{j, A}\right)_{, B}=\left(x_{j, B}\right)_{, A}=F_{j B, A}$. In other cases $(j=2,3)$, the same result can be obtained in the same way, and it can be concluded that

$$
[\operatorname{Div}(\operatorname{adj} \mathbf{F})]_{j}=(\operatorname{adj} \mathbf{F})_{j A, A}=0 \quad \text { for any } j
$$

Therefore, the right-hand side of Eq. (C.1.8) is expressed by

$$
\mathrm{RHS}=J\left(\pi^{i}-\operatorname{div} \boldsymbol{m}^{i}\right)
$$

By equating both expressions Eq. (C.1.9) and Eq. (C.1.11), the balance of mass in the current configuration is obtained as Eq. (C.1.4):

$$
\rightarrow \quad \frac{\partial \rho^{i}}{\partial t}+\operatorname{div}\left(\rho^{i} \boldsymbol{v}\right)=\pi^{i}-\operatorname{div} \boldsymbol{m}^{i} .
$$




\section{Bibliography}

[1] www.afexa.com/products/cell_fx/mechanisms.html. accessed on 23. February, 2012.

[2] WWw.arizonaorthopedicsurgeons.com/patient/patient-education/articular -cartilage-chondral-injuries/. accessed on 22. February, 2012.

[3] www.engin.umich.edu/class/bme456/cartilage/cart.htm. accessed on 20. February, 2012.

[4] www.orthspec.com/cartilage_sports_injuries.htm. accessed on 27. February, 2012.

[5] www.kneeindia.com/. accessed on 28. January, 2013.

[6] www.ottobuchinger.pwp.blueyonder.co.uk/mosaicpage.htm. accessed on 28. January, 2013.

[7] D. Ambrosi and F. Mollica. On the mechanics of a growing tumor. International Journal of Engineering Science, 40:1297-1316, 2002.

[8] Gerard A. Ateshian and Huiqun Wang. A theoretical solution for the frictionless rolling contact of cylindrical biphasic aricular carilage layers. Journal of Biomechanics, 28:13411355, 1995.

[9] Frank Baaijens, Carlijn Bouten, and Niels Driessen. Modeling collagen remodeling. Journal of Biomechanics, 43(1):166-175, 2010.

[10] Yavuz Başar and Dieter Weichert. Nonlinear Continuum Mechanics of Solids. Springer, 1999. 
[11] G. S. Beaupré, T. E. Orr, and D. R.Carter. An approach for time-dependent bone modeling and remodeling-application: A preliminary remodeling simulation. Journal of Orthopaedic Research.

[12] G. S. Beaupré, T. E. Orr, and D. R.Carter. An approach for time-dependent bone modeling and remodeling-theoretical development. Journal of Orthopaedic Research.

[13] A. Bellouquid and E. De Angelis. From kinetic models of multicellular growing systems to macroscopic biological tissue models. Nonlinear Analysis: Real World Applications, 12:1111-1122, 2011.

[14] Jean-Paul Boehler. Applications of tensor functions in solid mechanics. Springer, 1987.

[15] S. C. Cowin and W. C. Van Buskirk. Surface bone remodeling induced by a medullary pin. Journal of Biomechanics, 12:269-276, 1979.

[16] S. C. Cowin and D. H. Hegedus. Bone remodeling I: theory of adaptive elasticity. Journal of Elasticity, 6(3):313-326, 1976.

[17] Hilmi Demiray. A note on the elasticity of soft biological tissues. Journal of Biomechanics, 5:309-311, 1972.

[18] M. Destrade, M.D. Gilchrist, D.A. Prikazchikov, and G. Saccomandi. Surface instability of sheared soft tissues. Journal of Biomechanical Engineering, 130 (6):061107, 2008.

[19] A. Luis Dorfmann, William A. Woods Jr, and Barry A Trimmer. Muscle performance in a soft-bodied terrestrial crawler: constitutive modelling of strain-rate dependency. Journal Of The Royal Society, 5:349-362, 2008.

[20] Wolfgang Ehlers and Bernd Markert. A linear viscoelastic biphasic model for soft tissues based on the theory of porous media. Journal of Biomedical Engineering, 123:418-424, 2001.

[21] Alexander E. Ehret and Mikhail Itskov. Modeling of anisotropic softening phenomena: Application to soft biological tissues. International Journal of Plasticity, 25:901-919, 2009. 
[22] Marcelo Epstein and Gérard A. Maugin. Thermomechanics of volumetric growth in uniform bodies. International Journal of Plasticity, 16:951-978, 2000.

[23] Salvatore Federico, Alfio Grillo, Guido La Rosa, Gaetano Giaquinta, and Walter Herzo. A transversly isotropic, transversly homogeneous microstructural statistical model of articular cartilage. Journal of Biomechanics, 38:2008-2018, 2005.

[24] Alice J. Sophia Fox, Asheesh Bedi, and Scott A. Rodeo. The basic science of articular cartilage: Structure, composition, and function. SPORTS HEALTH, 1(6):461-468, 2009.

[25] Y. C. Fung. Biomechanics: Mechanical properties of living tissues. 1993.

[26] Y. C. Fung. Stress, strain, growth, and remodeling of living organisms. Z. angew. Math. Phys., 46 (Special issue):469-482, 1995.

[27] Zhan Gao, Kevin Lister, and Jaydev P. Desai. Constitutive modeling of liver tissue: Experiments and theory. Ann Biomed Eng., 38(2):505, 2010.

[28] José Jaime García and Daniel Humberto Cortés. A biphasic viscohyperelastic fibrilreinforced model for articular cartilage: Formulation and comparison with experimental data. Journal of Biomechanics, 40:1737-1744, 1984.

[29] José Jaime García and Daniel Humberto Cortés. A nonlinear biphasic viscohyperelasic model for aritcular cartialge. Journal of Biomechanics, 39:2991-2998, 2006.

[30] K. Garikipati, E.M. Arrunda, K. Grosh, H. Narayanan, and S. Calve. A continuum treatment of growth in biological tissue: the coupling of mass transport and mechanics. Journal of the mechanics and Physics of Solids, 52:1595-1627, 2004.

[31] Karsten Gavénis, Bernhard Schidt-Rohlfing, Stefan Andereya, Torsten Mumme, Ulich Schneider, and Ralf Müller-Rath. A cell-free collagen type I device for the treatment of focal cartilage defects. Artificial Organs, 34(1):79-83, 2010.

[32] D. H. Hegedus and S. C. Cowin. Bone remodeling II: small strain adaptive elasticity. Journal of Elasticity, 6(4):337-352, 1976. 
[33] M. H. Holmes and V. C. Mow. The nonlinear characteristics of soft gels and hydrated connective tissues in ultrafiltration. Journal of Biomechanics, 23(11):1145-1156, 1990.

[34] G. A. Holzapfel. Nonlinear Solid Mechanis - A Continuum Approach for Engineering. John Wiley \& Sons LTD, 2000.

[35] Gehard A. Holzapfel. On large strain viscoelasticity: Continuum formulation and finite element applications to elastomeric structures. International Journal for Numerical Methods in Engineering, 39(22):3903-3926, 1996.

[36] Gehard A. Holzapfel and Ray W. Ogden. Constitutive modelling of arteries. Proceedings of The Royal Society A, 466:1511-1597, 2010.

[37] R. Huiskes, H. Weinans, H. J. Grootenboer, M. Dalstra, and B.Fudala. Adaptive boneremodeling theory applied to prosthetic-design analysis. Journal of Biomechanics, 20:11351150, 1987.

[38] Mikhail Itskov. Tensor Algebra and Tensor Analysis for Engineers - With Applications to Continuum Mechanis. Springer, 2007.

[39] Mikhail Itskov. Lecture notes on Continuum Mechanis. Lehr- und Forschungsgebiet Kontinuumsmechanik, RWTH Aachen, 2010.

[40] Chistopher R. Jacobs, Juan C. Simo, Gary S. Beaupré, and Dennis R.Carter. Adaptive bone remodeling incorpoating simultaeous density and anisotropiy considerations. Journal of Biomechanics, 30. No. 6:603-613, 1997.

[41] Edward k. Rodriguez, Anne Hoger, and Andrew D. McCulloch. Stress-dependent finite growth in soft elastic tissues. Journal of Biomechanics, 27:455-467, 1994.

[42] T. Kaster, I. Sack, and A. Samani. Measurement of the hyperelastic properties of ex vivo brain tissue slices. Journal of Biomechanics, 44:1158-1163, 2001.

[43] Eberhard Klingbeil. Tensorrechnung für Ingenieure. Hochschultaschenbücher-Verlag, 1966.

[44] Stephen M. Klisch and Jeffrey C. Lotz. Application of a fiber-reinforced continumm theory to multiple deformations of annulus fibrosus. Journal of Biomechanics, 32:1027-1036, 1999. 
[45] Martin Kroon. A continuum mechanics framework and a constitutive model for remodelling of collagen gels and collagenous tissues. Journal of Mechanics and Physics of Solids, 58(6):918-933, 2010.

[46] Martin Kroon and Gehard A. Holzapfel. A new constitutive model for multi-layered collagenou tissues. Journal of Biomechanics, 41:2766-2771, 2008.

[47] Ellen Kuhl and Paul Steinmann. Mass- and volume-specific views on thermodynamics for open systems. Proc. R. Soc. Lond. A, 459:2547-2568, 2003.

[48] Michael K. Kwan, W. Michael Lai, and Van C. Mow. A finite deformation theory for cartilage and other soft hydrated connctive tissues-I. equilibrium results. Journal of Biomechanics, 23(2):145-155, 1990.

[49] Khanh Chau Le. Introduction to Continuum Mechanis. Lehrstuhl für Mechanik - Materialtheorie, Ruhr-Universitätet Bochum, 2003.

[50] J. Lemaitr and J.-L. Chaboche. Mechanics of solid materials. Springer, 1990.

[51] Georges Limbert and John Middleton. A transversely isotropic viscohyperelastic material application to the modeling of biological soft connective tissues. International Journal of Solids and Structures, 41:4237-4260, 2004.

[52] Yi Liu, Amy E. Kerdok, , and Robert D. Howe. A nonlinear finite element model of soft tissue indentation. Proceedings of Medical Simulation: International SymposiumISMC2004, 3078:67-76, 2004.

[53] Benjamin Loret and Fernando M.F. Simões. Elastic-growing tissue: A growth rate law that satisfies the dissipation inequality. Mechanics of Materials, 42:782-796, 2010.

[54] V. A. Lubarda and A. Hoger. On the mechanics of solids with a growing mass. International Journal of Solids and Structures, 39:4627-4664, 2002.

[55] A. F. Mak, W. M. Lai, and V. C. Mow. Biphasic indentation of articular cartilage-I theoretical analysis. Journal of Biomechanics, 20(7):703-714, 1987. 
[56] Andreas Menzel and Tobias Waffenschmidt. A microsphere-based remodelling formulation for anisotropic biological tissues. Philosophical Transactions of the Royal Society, 367:3499 3523, 2009.

[57] Karol Miller. How to test very soft biological tissues in extenxion? Journal of Biomechanics, 34:651-657, 2001.

[58] Rubem P. Mondaini and Panos M. Pardalos. Mathematical Modelling of Biosystems. Springer, 2008.

[59] Van C. Mow, Mark H. Holmes, and W. Michael Lai. Fluid transport and mechanical properties of aritcular cartilage: a review. Journal of Biomechanics, 17(5):377-394, 1984.

[60] Van C. Mow and Anthony Ratcliffe. Cartilage and diarthrodial joints as paradigms for hierarchical materials and structures. Biomaterials, 13:No.2 67-97, 1992.

[61] R. Müller-Rath, K. Gavénis, S. Andereya, T. Mumme, B. Smidt-Rohlfing, and U. Schneider. A novel rat tail collagen type-i gel for the cultivation of human articular chondrocytes in low cell density. The international journal of artificial organs, 30(12):1057-1067, 2007.

[62] Steve Mwenifumbo, Milo S. Shaffer, and Molly M. Stevens. Exploring cellular behavior with multi-walled carbon nanotube constructs. Journal of Materials Chemistry, 17:18941902, 2007.

[63] S. Nebelung, A. Ladenburger, K. Gavénis, M.Stoffel, S. Andereya, and R. MüllerRath. Tissue engineering von knorpelersatzgewebe-mechanische stimulation in der invitro-kulivierung von humanen chondrozyten. Z Orthop Unfall, 148:1-9, 2010.

[64] R.W. Ogden. Nonlinear elasticity, anisotropy, material stability and residual stresses in soft tissue. Biomechanics of Soft Tissue in Cardiovascular Systems, CISM Courses and Lectures Series no. 441:65-108, 2003.

[65] C.W.J. Oomens, O.F.J.T. Bressers, E.M.H. Bosboom, C.V.C. Bouten, and D.L. Bader. Can loaded interface characteristics influence strain distributions in muscle adjacent to bony prominences? Computer Methods in Biomechanics and Biomedical Engineering, 6, No.3:171-180, 2003. 
[66] C.W.J. Oomens and D.H.Campen. A mixture approch to the mechanics of skin. Journal of Biomechanics, 20. No. 9:877-885, 1987.

[67] Dominique P. Pioletti and Lalao R. Rakotomanana. Non-linear viscoelastic laws for soft tissues. European Journal of Mechanics - A/Solids, 19:749-759, 2000.

[68] T. Ricken and J. Bluhm. Evolutional growth and remodeling in multiphase living tisssue. Computational Material Science, 45:806-811, 2009.

[69] Tim Ricken, Alexander Schwarz, and Joachim Bluhm. A triphasic theory for growth in biological tissue - basics and applications. Mat.-wiss. u. Werkstofftech., 37 (6):446-456, 2006.

[70] Tim Ricken, Alexander Schwarz, and Joachim Bluhm. A triple model of transversely isotropic bioloical tissue with applications to stress and biological induced grwoth. Computational Material Science, 39:124-136, 2007.

[71] Hart RT, Davy Dt, and Heiple KG. A computational method for stress analysis of adaptive elastic materials with a view toward applications in strain-induced bone remodeling. Journal of Biomechanical Engineering, 106:342-350, 1984.

[72] Tamer El Sayed, Alejanodro Mota, Fernando Fraternali, and Michael Ortiz. A variational constitutive model for soft biological tissues. Journal of Biomechanics, 41:1458-1466, 2008.

[73] R. Skalak, G. Dasgupta, M. Moss, E. Otten, P. Dullemeijer, and H. Vilmann. Analytical description of growth. Journal of theoretical Biology, 94:555-577, 1982.

[74] Robert L. Spilker, Peter S. Donzelli, and Van. C. Mow. A transversely isotropic biphasic finite element model of the meniscus. Journal of Biomechanics, 25(9):1027-1045, 1992.

[75] M. Stoffel, J.H. Yi, D. Weichert, and K. Gavenis R. Müller-Rath. A biomechanical model for cartilage replacement material. BIOmaterialien, 10:61-62, 2009.

[76] Marcus Stoffel, Dieter Weichert, and Ralf Müller-Rath. Modeling of articular cartilage replacement materials. Archives of mechanics, 61,1:69-87, 2009. 
[77] Marcus Stoffel, Jeong Hun Yi, Dieter Weichert, Karsten Gavenis, and Ralf MüllerRath. Biomechanical modelling of cellular articular cartilage replacement tissues. PAMM, 10,1:95-96, 2010.

[78] Marcus Stoffel, Jeong-Hun Yi, Dieter Weichert, Bei Zhou, Sven Nebelung, Ralf MüllerRath, and Karsten Gavenis. Bioreactor cultivation and remodelling simulation for cartilage replacement material. Medical Engineering \& Physics, 34:56-63, 2012.

[79] Larry A. Taber. Biomechanics of growth, remodeling, and morphogenesis. Applied Mechanics Reviews, 48:487-545, 1995.

[80] José J. Muñoz, Vito Conte, and Mark Miodownik. Stress-dependent morphogenesis: continuum mechanics and truss systems. Biomechanics and Modeling in Mechanobiology, 9(4):451-467, 2010.

[81] Kerem Ün and Robert L. Spilker. Finite element simulation of biphasic soft tissue conatct with application to the shoulder joint. Proceedings of the 23rd Annual EMBS International Conference, October 25-28:1504-1507, 2001.

[82] P. Vena, d. Gastaldi, L. Socci, and G. Pennati. An anisotropic model for tissue growth and remodeling during early development of cerebral aneurysms. Computational Material Science, 43:565-577, 2008.

[83] M. Vogler, S. Kolling, and R. Rolfes. Transversely isotropic plasticity wiht application to fiber-reinforced plastics. LS-DYNA Anwenderforum, Frankelthal, Material II:D-II 55-74, 2007.

[84] Konstantin Y. Volokh. Tissue morphogenesis: a surface buckling mechanism. International Journal of Developmental Biology, 50:359-365, 2006.

[85] W. Wilson, C.C. van Donkelaar, B. van Rietbergen, K. Ito, and R. Huiskes. Stresses in the local collagen network of articular cartilage: a poroviscoelastic fibril-reinforced finite element study. Journal of Biomechanics, 37:357-366, 2004. 
[86] J.Z. Wu, W. Herzog, and M. Epstein. Evaluation of the finite element software ABAQUS for biomechanical modelling of biphasic tissues. Journal of Biomechanics, 31:165-169, 1998.

[87] Jeong Hun Yi, Marcus Stoffel, Dieter Weichert, Sven Nebelung, and Ralf Müller-Rath. Experimental and numerical investigations on engineering tissue enhanced by mechanical stimulation. PAMM, 11,1:91-92, 2011.

[88] Jeong Hun Yi, Marcus Stoffel, Dieter Weichert, Sven Nebelung, and Björn Rath. Experimental and theoretical investigation on soft tissue remodeling enhanced by cell activity. PAMM, 12,1:87-88, 2012.

[89] J.H. Yi, M. Stoffel, D. Weichert, and K. Gavenis R. Müller-Rath. Theoretical and numerical investigations of remodelling of cellular material. AIMM, pages 1135-1143, 2010.

[90] J.H. Yi, M. Stoffel, D. Weichert, and K. Gavenis R. Müller-Rath. Theoretical investigation of remodelling of cellular materials at macro- and micro level. Advanced Problems in Mechanics of Heterogeneous Media and Thin-Walled Structures, pages 351-358, 2010.

[91] Bei Zhou, Marcus Stoffel, Dieter Weichert, and Karsten Gavénis. Identification of diffusion properties of replacement materials for articular cartilage using numerical simulation. Proceedings of the 1st International Symposium on Numerical Simulation in Orthopaedic Biomechanics - SimoOtho, -:111-116, 2011.

[92] Bei Zhou, Marcus Stoffel, Dieter Weichert, and Björn Rath. Theoretical and experimental investigation of cartilage replacement material for medical applifications. PAMM, 12:95-96, 2012. 